2. To: (Receiving Organization)

Distribution

5. Proj./Prog./Dept./Div.:

Tank 241-U-108/Waste

Management/DAI/Process

Engineering

8. Originator Remarks:

This document is being released into the supporting document system for retrievability purposes.

11. Receiver Remarks:

For release.
3. From: (Originating Organization)

Data Assessment and Interpretation

6. Design Authority/ Design Agent/Cog. Engr.:

Kevin E. Bell
4. Related EDT No_:

$\mathrm{N} / \mathrm{A}$

7. Purchase Order No.:

N/A

9. Equip./Component No.:

N/A

10. System/Bldg./Facility:

241-U-108

12. Major Assm. Dwg. No.:

$\mathrm{N} / \mathrm{A}$

13. Permit/Permit Application No.: $\mathrm{N} / \mathrm{A}$

14. Required Response Date: $03 / 12 / 97$

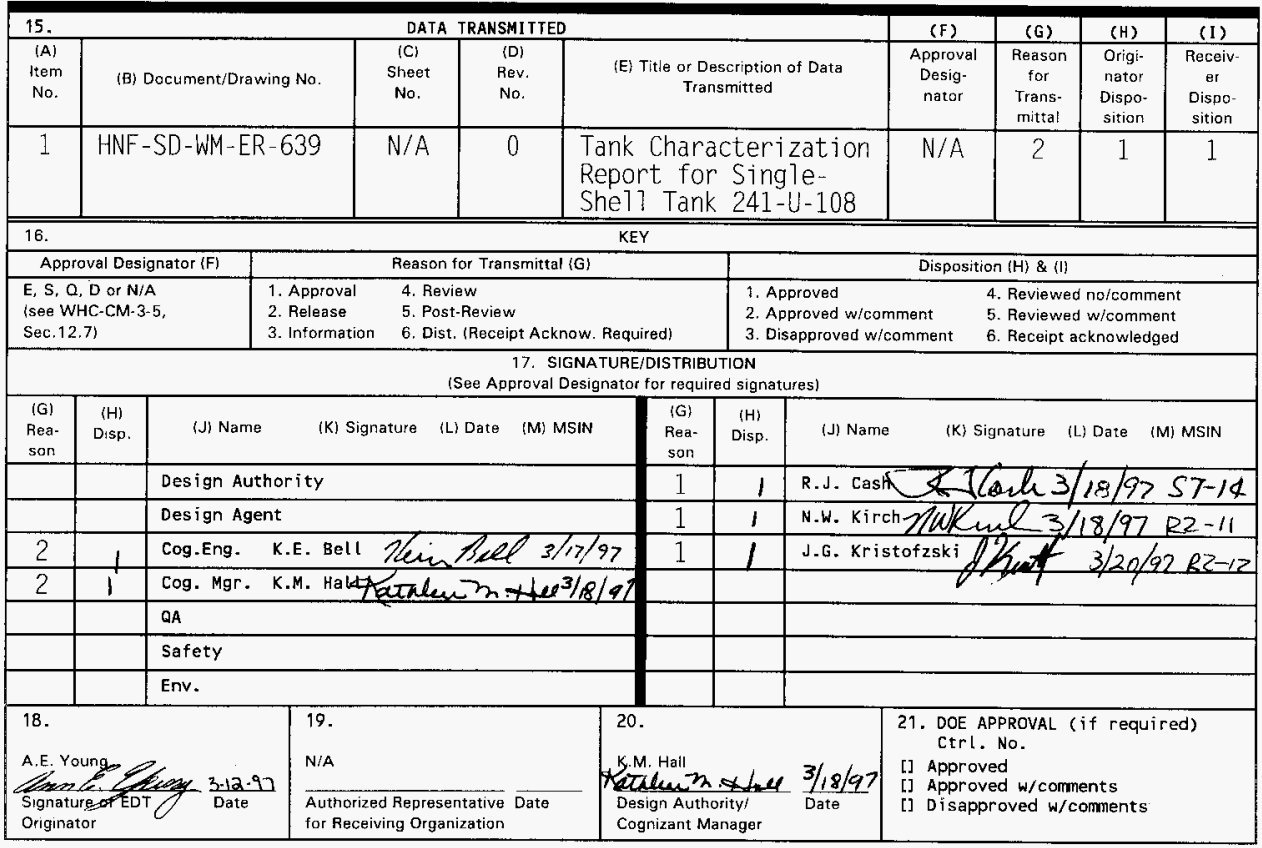

BD-7400-172-2(05/96) GEFO97 


\section{Tank Characterization Report for Single-Shell Tank 241-U-108}

Kevin E. Bell

Lockheed Martin Hanford Corp., Richland. WA 99352

U.S. Department of Energy Contract DE-AC06-87RL10930

EDT/ECN: EDT-617587 UC: 2070

Org Code: 74620

B\&R Code: EW 3120074 Total Pages:-354

Key Words: Waste Characterization. Single-Shell Tank. SST, Tank 241-U108, Tank U-108, U-108, U Farm, Tank Characterization Report, TCR, Waste Inventory, TPA Mi lestone M-44

Abstract: This document summarizes the information on the historical uses. present status, and the sampling and analysis results of waste stored in Tank 241-U-108. This report supports the requirements of the Tri-Party Agreement Mi lestone M-44-10.

TRADEMARK DISCLAIMER. Reference here in to any specific commercial product, process, or service by trade name, trademark, manufacturer, or otherwise, does not necessarily constitute or imply its endorsement, recomendation, or favoring by the United States Government or any agency thereof or its contractors or subcontractors.

Printed in the United States of America. To obtain copies of this document, contact: WHC/BCS Document Control Services, P.O. Box 1970, Mailstop H6-08, Richland WA 99352, Phone (509) 372-2420; Fax (509) 376-4989.
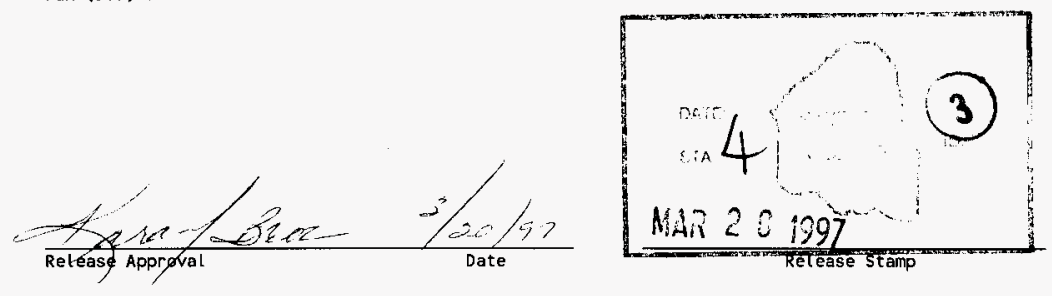

\section{Approved for Public Release}


UC-2070

\section{Tank Characterization Report for Single-Shell Tank 241-U-108}

K. E. Bell

Lockheed Martin Hanford Corporation

R. D. Cromar

Numatec Hanford Corporation

Date Published

March 1997

Prepared for the U.S. Department of Energy

Assistant Secretary for Environmental Management

Project Hanford Management Contractor for the

U.S. Department of Energy under Contract DE-AC06-96RL13200

Approved for public release; distribution is unlimited 


\section{CONTENTS}

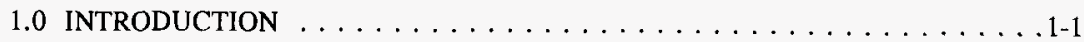

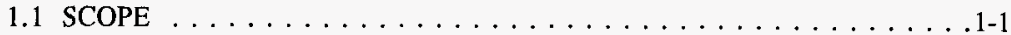

1.2 TANK BACKGROUND $\ldots \ldots \ldots \ldots \ldots \ldots \ldots \ldots \ldots \ldots \ldots \ldots \ldots \ldots \ldots .2$

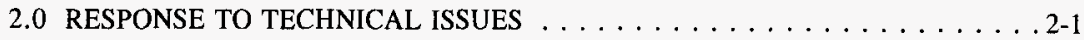

2.1 SAFETY SCREENING AND ORGANIC COMPLEXANT EVALUATION . 2-2

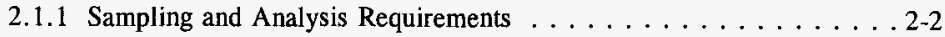

2.1.2 Exothermic Conditions (Energetics) $\ldots \ldots \ldots \ldots \ldots \ldots \ldots \ldots$

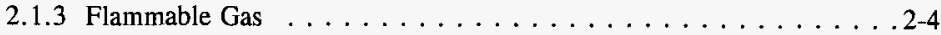

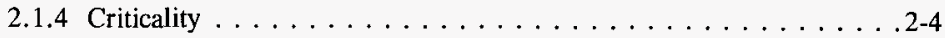

2.1.5 Total Organic Carbon Content $\ldots \ldots \ldots \ldots \ldots \ldots \ldots . . .2-4$

2.1.6 Moisture Content . . . . . . . . . . . . . . . 2-4

2.2 HISTORICAL EVALUATION $\ldots \ldots \ldots \ldots \ldots \ldots \ldots \ldots \ldots \ldots .2-5$

2.3 WASTE COMPATIBILITY EVALUATION $\ldots \ldots \ldots \ldots \ldots \ldots \ldots .6 \ldots$

2.4 TANK VAPOR EVALUATION $\ldots \ldots \ldots \ldots \ldots \ldots \ldots \ldots \ldots \ldots$

2.4 .1 Permanent Gases ... . . . . . . . . . . . . 2-11

2.4.2 Total Non-Methane Hydrocarbons . . . . . . . . . . . 2-11

2.4 .3 Volatile Organic Analysis . . . . . . . . . . . . 2-12

2.4.4 Semi-Volatile Organic Analyses . . . . . . . . . . . 2-12

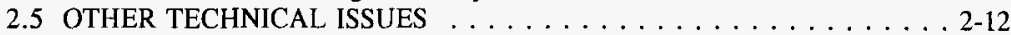

2.6 SUMMARY $\ldots \ldots \ldots \ldots \ldots \ldots \ldots \ldots \ldots \ldots \ldots \ldots \ldots \ldots \ldots \ldots \ldots \ldots .12$

3.0 BEST-BASIS INVENTORY ESTIMATE $\ldots \ldots \ldots \ldots \ldots \ldots \ldots$ 3-1

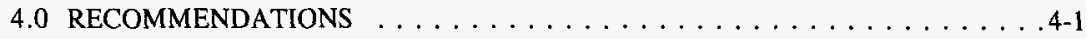

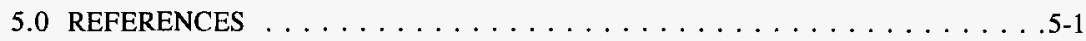

APPENDIXES

APPENDIX A: HISTORICAL TANK INFORMATION $\ldots \ldots \ldots \ldots \ldots \ldots$ A-1

A1.0 CURRENT TANK STATUS $\ldots \ldots \ldots \ldots \ldots \ldots \ldots \ldots \ldots$ A-3

A2.0 TANK DESIGN AND BACKGROUND $\ldots \ldots \ldots \ldots \ldots \ldots \ldots$ A-4

A3.0 PROCESS KNOWLEDGE $\ldots \ldots \ldots \ldots \ldots \ldots \ldots \ldots \ldots \ldots$ A-8

A3.1 WASTE TRANSFER HISTORY $\ldots \ldots \ldots \ldots \ldots \ldots \ldots \ldots$ A-8

A3.2 HISTORICAL ESTIMATION OF TANK CONTENTS $\ldots \ldots \ldots \ldots$ A-8 


\section{CONTENTS (Continued)}

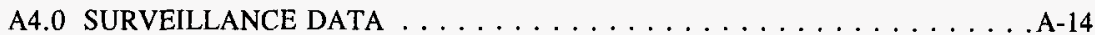

A4.1 SURFACE-LEVEL READINGS $\ldots \ldots \ldots \ldots \ldots \ldots \ldots \ldots \ldots \ldots$. . . . . . . . . .

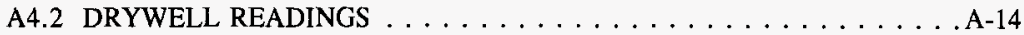

A4.3 INTERNAL TANK TEMPERATURES $\ldots \ldots \ldots \ldots \ldots \ldots \ldots \ldots$ A-14

A4.4 TANK $241-\mathrm{U}-108$ PHOTOGRAPHS . . . . . . . . . . . . . . . A-16

A5.0 APPENDIX A REFERENCES $\ldots \ldots \ldots \ldots \ldots \ldots \ldots \ldots \ldots$ A-18

APPENDIX B: SAMPLING OF TANK $241-\mathrm{U}-108 \ldots \ldots \ldots \ldots \ldots \ldots$ B-1

B1.0 TANK SAMPLING OVERVIEW $\ldots \ldots \ldots \ldots \ldots \ldots \ldots \ldots$ B-3

B1.1 DESCRIPTION OF 1996 CORE AND 1995 GRAB SAMPLING EVENTS B-4

B1.2 SAMPLE HANDLING (1996 CORE AND 1995 GRAB SAMPLES) . . . . B-4

B1.3 SAMPLE ANALYSIS (1996 CORE AND 1995 GRAB SAMPLES) . . . . . B-12

B1.4 DESCRIPTION OF 1995 VAPOR SAMPLING . . . . . . . . . . B B-20

B1.5 SAMPLE HANDLING AND ANALYSIS (1995 VAPOR SAMPLES) . . . B-21

B1.6 HISTORICAL SAMPLING EVENTS $\ldots \ldots \ldots \ldots \ldots \ldots \ldots \ldots$. . 22

B1.6.1 Description of Historical Sampling Events $\ldots \ldots \ldots \ldots \ldots$ B-22

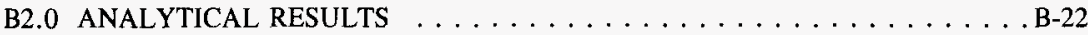

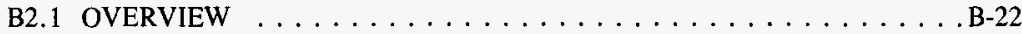

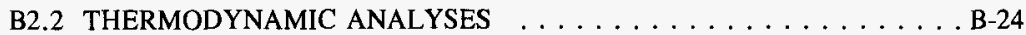

B2.2.1 Thermogravimetric Analysis . . . . . . . . . . . . . . B-24

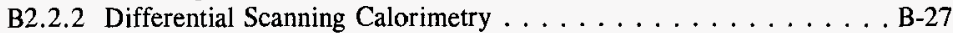

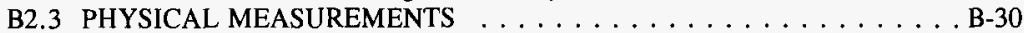

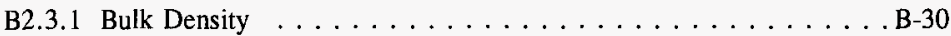

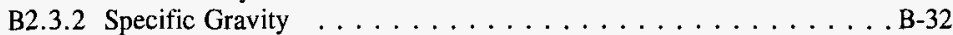

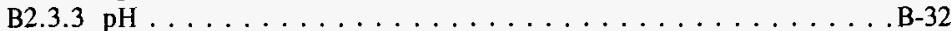

B2.4 INORGANIC ANALYSES $\ldots \ldots \ldots \ldots \ldots \ldots \ldots \ldots \ldots \ldots$ B-33 $\ldots \ldots \ldots \ldots$

B2.4.1 Total Organic Carbon . . . . . . . . . . . . B-33

B2.4.2 Total Inorganic Carbon $\ldots \ldots \ldots \ldots \ldots \ldots \ldots \ldots \ldots$. . . . . . . . . $\ldots \ldots$

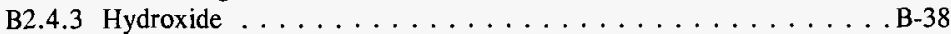

B2.4.4 Inductively Coupled Plasma $\ldots \ldots \ldots \ldots \ldots \ldots \ldots \ldots$ B-38

B2.4.5 Ion Chromatography . . . . . . . . . . . . . . . B-74

B2.5 RADIOCHEMICAL ANALYSES $\ldots \ldots \ldots \ldots \ldots \ldots \ldots \ldots$ B-92

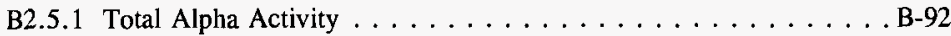

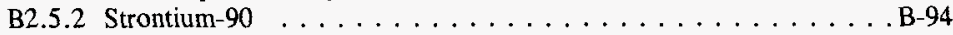

B2.5.3 Total Beta . . . . . . . . . . . . . . . . . . . . . . . B-97

B2.5.4 Total Uranium . . . . . . . . . . . . . . . . . B-97

B2.5.5 Plutonium-239/240 . . . . . . . . . . . . . . . . . . . B-98

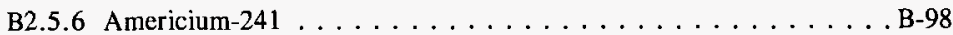




\section{CONTENTS (Continued)}

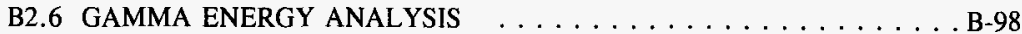

B2.6.1 Cesium-137 . . . . . . . . . . . . . . . . . . . B-99

B2.7 VAPOR PHASE MEASUREMENTS $\ldots \ldots \ldots \ldots \ldots \ldots \ldots \ldots$ B-108

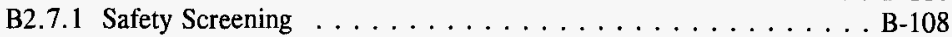

B2.7.2 1995 Tank Vapor Samples . . . . . . . . . . . . . . B-109

B2.7.3 Flammable Gas Monitoring . . . . . . . . . . . . . B-112

B2.8 HISTORICAL SAMPLE RESULTS $\ldots \ldots \ldots \ldots \ldots \ldots \ldots \ldots \ldots$ B-112

B3.0 ASSESSMENT OF CHARACTERIZATION RESULTS $\ldots \ldots \ldots \ldots \ldots$. . . . . 116

B3.1 FIELD OBSERVATIONS $\ldots \ldots \ldots \ldots \ldots \ldots \ldots \ldots \ldots$. . . . . . . . .

B3.2 QUALITY CONTROL ASSESSMENT $\ldots \ldots \ldots \ldots \ldots \ldots \ldots$. . . . . . . $\ldots$

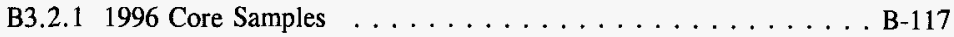

B3.2.2 1995 Grab Samples . . . . . . . . . . . . . . . . . . . . B-118

B3.3 DATA CONSISTENCY CHECKS . . . . . . . . . . . . . . B-119

B3.3.1 Comparison of Results from Different Analytical Methods . . . . B-119

B3.3.2 Mass and Charge Balance $\ldots \ldots \ldots \ldots \ldots \ldots \ldots$ B-120

B3.3.3 Total Beta Results . . . . . . . . . . . . . . . . . . . B-122

B3.3.4 Comparison of 1996 Core Drainable Liquid and 1995 Grab Sample Results . . . . . . . . . . . . . B-122

B3.3.5 Comparison of TOC by Persulfate and TOC by Furnace Oxidation B-123

B3.3.6 Comparison of Transfer History and Analytical Results . . . . . B- 123

B3.4 MEAN CONCENTRATIONS AND CONFIDENCE INTERVALS . . . . B-123

B3.4.1 Composite, Solid Segment, and Liquid Segment Means . . . . . B-125

B3.4.2 Analysis of Variance Models . . . . . . . . . . . . . . B-131

B3.4.3 Inventory . . . . . . . . . . . . . . . B-133

B4.0 APPENDIX B REFERENCES $\ldots \ldots \ldots \ldots \ldots \ldots \ldots \ldots$. . . . . . . . . . . . . .

APPENDIX C: STATISTICAL ANALYSIS FOR ISSUE RESOLUTION $\ldots \ldots \ldots$ C-1

C1.0 STATISTICS FOR SAFETY SCREENING AND ORGANIC DQOS $\ldots \ldots$ C-3

C1.1 STATISTICS FOR THE ORGANIC DQO . . . . . . . . . . C-7

C2.0 APPENDIX C REFERENCES $\ldots \ldots \ldots \ldots \ldots \ldots \ldots \ldots \ldots \ldots$ C-12 


\section{CONTENTS (Continued)}

APPENDIX D: EVALUATION TO ESTABLISH BEST-BASIS

INVENTORY FOR TANK $241-U-108 \ldots \ldots \ldots \ldots \ldots \ldots$ D-1

D1.0 CHEMICAL INFORMATION SOURCES $\ldots \ldots \ldots \ldots \ldots \ldots \ldots$ D-3

D2.0 COMPARISON OF COMPONENT INVENTORY VALUES $\ldots \ldots \ldots \ldots$ D-3

D3.0 COMPONENT INVENTORY EVALUATION $\ldots \ldots \ldots \ldots \ldots \ldots$ D-6

D3.1 CONTRIBUTING WASTE TYPES $\ldots \ldots \ldots \ldots \ldots \ldots \ldots$ D-6

D3.2 EVALUATION OF PROCESS FLOWSHEET INFORMATION $\ldots \ldots \ldots$ D-7

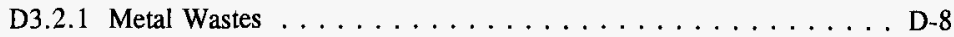

D3.2.2 REDOX Cladding Waste $\ldots \ldots \ldots \ldots \ldots \ldots \ldots \ldots$. . . . . . . .

D3.2.3 Saltcake . . . . . . . . . . . . . . . . . . D-9

D3.3 DOCUMENT ELEMENT BASIS $\ldots \ldots \ldots \ldots \ldots \ldots \ldots \ldots$ D-11

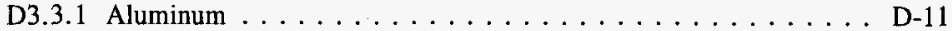

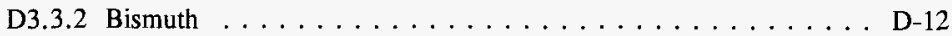

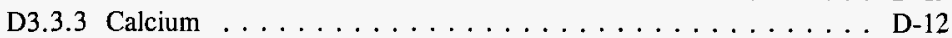

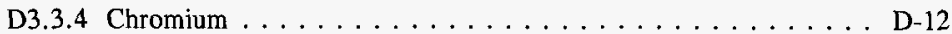

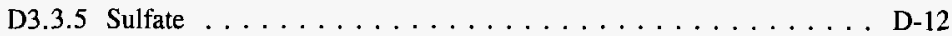

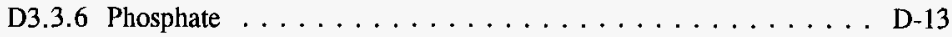

D4.0 ESTABLISH THE BEST BASIS AND ESTABLISH

COMPONENT INVENTORIES $\ldots \ldots \ldots \ldots \ldots \ldots \ldots \ldots \ldots$ D-13

D5.0 APPENDIX D REFERENCES $\ldots \ldots \ldots \ldots \ldots \ldots \ldots \ldots \ldots \ldots$ D-18

APPENDIX E: BIBLIOGRAPHY FOR TANK $241-U-108 \ldots \ldots \ldots \ldots \ldots$ E-1 


\section{LIST OF FIGURES}

A2-1 Riser Configuration for Tank $241-\mathrm{U}-108 \ldots \ldots \ldots \ldots \ldots \ldots$ A-5

A2-2 Tank 241-U-108 Cross Section and Schematic $\ldots \ldots \ldots \ldots \ldots \ldots$ A-7

A3-1 Tank Layer Model $\ldots \ldots \ldots \ldots \ldots \ldots \ldots \ldots \ldots \ldots \ldots$. . . . . . . .

A4-1 Level History for Tank $241-\mathrm{U}-108 \ldots \ldots \ldots \ldots \ldots \ldots \ldots$. . . . . . . . . . . . . .

A4-2 Weekly High Temperature Plot for Tank $241-\mathrm{U}-108 \ldots \ldots \ldots \ldots$. . . . A-17

\section{LIST OF TABLES}

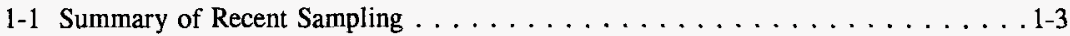

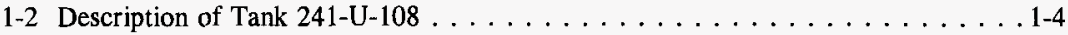

2-1 Expected and Measured Concentrations for Tank 241-U-108 Waste Types . . . 2-7

2-2 Decision Variables and Criteria for the Waste Compatibility Data Quality Objective $\ldots \ldots \ldots \ldots \ldots \ldots \ldots \ldots \ldots .2-8$

2-3 Summary of DQO and Test Plan Evaluations $\ldots \ldots \ldots \ldots \ldots \ldots \ldots \ldots$ 2-14

3-1 Best-Basis Inventory Estimates for Nonradioactive Components

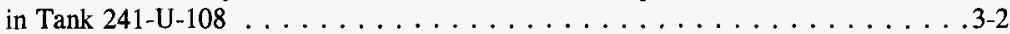

3-2 Best-Basis Inventory Estimates for Radioactive Components

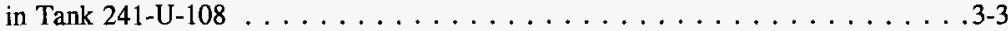

4-1 Acceptance of Tank 241-U-108 Sampling and Analysis $\ldots \ldots \ldots \ldots \ldots$. . . . . .

4-2 Acceptance of Evaluation of Characterization Data and Information for Tank $241-\mathrm{U}-108 \ldots \ldots \ldots \ldots$. . . . . . . . . . . . . . . . . . .

A1-1 Tank Contents Status Summary $\ldots \ldots \ldots \ldots \ldots \ldots \ldots \ldots$ A -4

A2-1 Tank 241-U-108 Risers $\ldots \ldots \ldots \ldots \ldots \ldots \ldots \ldots \ldots \ldots$ A-6

A3-1 Tank 241-U-108 Major Transfers $\ldots \ldots \ldots \ldots \ldots \ldots \ldots \ldots \ldots \ldots$ A-9 


\section{LIST OF TABLES (Continued)}

A3-2 Historical Tank Inventory Estimate . . . . . . . . . . . . . . . A-12

B1-1 Integrated Data Quality Objective Requirements for Tank 241-U-108 . . . . . B-5

B1-2 Sample Receipt and Extrusion Information for Tank 241-U-108, Core 141 , Riser $7 \ldots \ldots \ldots \ldots \ldots \ldots \ldots \ldots$

B1-3 Sample Receipt and Extrusion Information for Tank 241-U-108, Core 145, Riser $9 \ldots \ldots \ldots \ldots \ldots \ldots \ldots$

B1-4 Sample Receipt and Extrusion Information for Tank 241-U-108, Core 146, Riser $2 \ldots \ldots \ldots \ldots \ldots \ldots \ldots$

B1-5 Tank 241-U-103 Sample Analysis Summary . . . . . . . . . . . . . . . . B-13

B1-6 Tank 241-U-108 Gas and Vapor Sample Type and Number for the 1995 Tank 241-U-108 Vapor Samples . . . . . . . . . . . . . . . . B-21

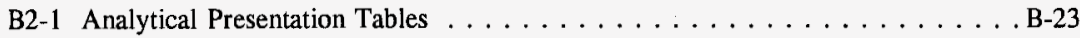

B2-2 Tank 241-U-108 Core Sample Analytical Results: Percent Water (TGA) . . . . B-25

B2-3 Tank 241-U-108 Grab Sample Analytical Results: Percent Water (TGA) . . . . B-27

B2-4 Tank 241-U-108 Core Sample Analytical Results: Exotherm -

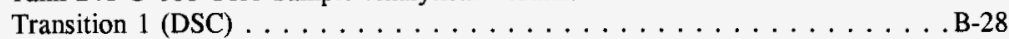

B2-5 Tank 241-U-108 Grab Sample Analytical Results: Exotherm - Transition 1 . . B-29

B2-6 Tank 241-U-108 Core Sample Analytical Results: Bulk Density . . . . . . . . B-30

B2-7 Tank 241-U-108 Analytical Results: Specific Gravity . . . . . . . . . . . . B-32

B2-8 Tank 241-U-108 Grab Sample Analytical Results: Specific Gravity . . . . . . . B-32

B2-9 Tank 241-U-108 Grab Sample Analytical Results: pH Measurement . . . . . B-32

B2-10 Tank 241-U-108 Core Sample Analytical Results: Total Organic Carbon (Persulfate) $\ldots \ldots \ldots \ldots \ldots \ldots \ldots \ldots$. . . . . . . . . . . . . . . . . 


\section{LIST OF TABLES (Continued)}

B2-11 Tank 241-U-108 Grab Sample Analytical Results: Total Organic Carbon

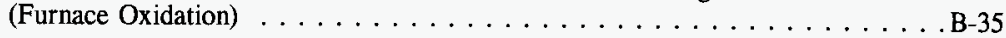

B2-12 Tank 241-U-108 Core Sample Analytical Results: Total Inorganic Carbon . . B-36

B2-13 Tank 241-U-108 Grab Sample Analytical Results: Total Inorganic Carbon . . . B-38

B2-14 Tank 241-U-108 Grab Sample Analytical Results: Hydroxide . . . . . . . . . B-38

B2-15 Non-Detected ICP Analytes for Tank 241-U-108 Core Samples . . . . . . . B-39

B2-16 Tank 241-U-108 Core Sample Analytical Results: Aluminum (ICP) . . . . . B-41

B2-17 Tank 241-U-108 Grab Sample Analytical Results: Aluminum (ICP) . . . . . B-43

B2-18 Tank 241-U-108 Core Sample Analytical Results: Antimony (ICP) . . . . . . B B-44

B2-19 Tank 241-U-108 Core Sample Analytical Results: Boron (ICP) . . . . . . . . B-45

B2-20 Tank 241-U-108 Core Sample Analytical Results: Cadmium (ICP) . . . . . . B-46

B2-21 Tank 241-U-108 Core Sample Analytical Results: Calcium (ICP) . . . . . . . B-47

B2-22 Tank 241-U-108 Core Sample Analytical Results: Chromium (ICP) . . . . B B-48

B2-23 Tank 241-U-108 Core Sample Analytical Results: Copper (ICP) . . . . . . B-51

B2-24 Tank 241-U-108 Core Sample Analytical Results: Iron (ICP) . . . . . . . B-52

B2-25 Tank 241-U-108 Grab Sample Analytical Results: Iron (ICP) . . . . . . . . B-54

B2-26 Tank 241-U-108 Core Sample Analytical Results: Lithium (ICP) . . . . . . . B-54

B2-27 Tank 241-U-108 Core Sample Analytical Results: Manganese (ICP) . . . . . B-55

B2-28 Tank 241-U-108 Core Sample Analytical Results: Molybdenum (ICP) . . . . . B-58

B2-29 Tank 241-U-108 Core Sample Analytical Results: Nickel (ICP) . . . . . . . B B-59

B2-30 Tank 241-U-108 Core Sample Analytical Results: Phosphorus (ICP) . . . . . . B-62

B2-31 Tank 241-U-108 Core Sample Analytical Results: Potassium (ICP) . . . . . . . B-65 


\section{LIST OF TABLES (Continued)}

B2-32 Tank 241-U-108 Core Sample Analytical Results: Silicon (ICP) . . . . . . . . B-66

B2-33 Tank 241-U-108 Core Sample Analytical Results: Silver (ICP) . . . . . . . B-69

B2-34 Tank 241-U-108 Core Sample Analytical Results: Sodium (ICP) . . . . . . . B-70

B2-35 Tank 241-U-108 Grab Sample Analytical Results: Sodium (ICP) . . . . . . . B-73

B2-36 Tank 241-U-108 Core Sample Analytical Results: Sulfur (ICP) . . . . . . . B B-73

B2-37 Tank 241-U-108 Core Sample Analytical Results: Zinc (ICP) . . . . . . . . B-74

B2-38 Tank 241-U-108 Core Sample Analytical Results: Chloride (IC) . . . . . . B-75

B2-39 Tank 241-U-108 Grab Sample Analytical Results: Chloride (IC) . . . . . . B-77

B2-40 Tank 241-U-108 Core Sample Analytical Results: Fluoride (IC) . . . . . . B-78

B2-41 Tank 241-U-108 Grab Sample Analytical Results: Fluoride (IC) . . . . . . . B-80

B2-42 Tank 241-U-108 Core Sample Analytical Results: Nitrate (IC) . . . . . . . . B-80

B2-43 Tank 241-U-108 Grab Sample Analytical Results: Nitrate (IC) . . . . . . . B-82

B2-44 Tank 241-U-108 Core Sample Analytical Results: Nitrite (IC) . . . . . . . . B-83

B2-45 Tank 241-U-108 Grab Sample Analytical Results: Nitrite (IC) . . . . . . . . B-85

B2-46 Tank 241-U-108 Core Sample Analytical Results: Phosphate (IC) . . . . . . . B-85

B2-47 Tank 241-U-108 Grab Sample Analytical Results: Phosphate (IC) . . . . . . . B B-87

B2-48 Tank 241-U-108 Core Sample Analytical Results: Sulfate (IC) . . . . . . . . B B-88

B2-49 Tank 241-U-108 Grab Sample Analytical Results: Sulfate (IC) . . . . . . . B-90

B2-50 Tank 241-U-108 Core Sample Analytical Results: Oxalate (IC) . . . . . . . B-90

B2-51 Tank 241-U-108 Core Sample Analytical Results: Total Alpha . . . . . . . . B-92

B2-52 Tank 241-U-108 Core Sample Analytical Results: Strontium-89/90 . . . . . . B-95 


\section{LIST OF TABLES (Continued)}

B2-53 Tank 241-U-108 Grab Sample Analytical Results: Strontium-89/90 . . . . . . B-96

B2-54 Tank 241-U-108 Core Sample Analytical Results: Total Beta . . . . . . . . . B-97

B2-55 Tank 241-U-108 Core Sample Analytical Results: Total Uranium . . . . . . . B-97

B2-56 Tank 241-U-108 Grab Sample Analytical Results: Plutonium-239/40 . . . . . B-98

B2-57 Tank 241-U-108 Grab Sample Analytical Results: Americium-241 . . . . . B-98

B2-58 Tank 241-U-108 Core Sample Analytical Results: Cesium-137 (GEA) . . . . . B-99

B2-59 Tank 241-U-108 Grab Sample Analytical Results: Cesium-137 (GEA) . . . . B-101

B2-60 Tank 241-U-108 Core Sample Analytical Results: Americium-241 (GEA) . . . B-101

B2-61 Tank 241-U-108 Core Sample Analytical Results: Cobalt-60 (GEA) . . . . B-103

B2-62 Tank 241-U-108 Core Sample Analytical Results: Europium-154 (GEA) . . B-105

B2-63 Tank 241-U-108 Core Sample Analytical Results: Europium-155 (GEA) . . B-107

B2-64 Results of Vapor Phase Measurements of Tank 241-U-108 . . . . . . . . B-109

B2-65 Vapor Analytical Summary for Tank 241-U-108 . . . . . . . . . . . B-111

B2-66 Tank 241-U-108 Flammable Gas Monitoring Vapor Sample Results . . . . . B-112

B2-67 1971 Sample $\ldots \ldots \ldots \ldots \ldots \ldots \ldots \ldots \ldots \ldots \ldots \ldots \ldots$

B2-68 1973 or 1974 Supernatant Sample . . . . . . . . . . . . . . . . . . B-113

B2-69 1975 Sludge Sample . . . . . . . . . . . . . . . . . B-115

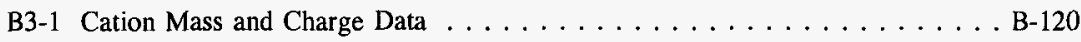

B3-2 Anion and Neutral Species Mass and Charge Data . . . . . . . . . . B-121

B3-3 Mass Balance Totals . . . . . . . . . . . . . . . . . . . B-121

B3-4 Comparison of 1995 Grab and 1996 Drainable Liquid Analytical Results . . . B-124 


\section{LIST OF TABLES (Continued)}

B3-5 Comparison of Historical Tank Content Estimate with 1996 Core Sample Analytical Results from Tank 241-U-108

B3-6 95 Percent Two-Sided Confidence Interval for the Mean Concentration for Composite Sample Data . . . . . . . . . . . . . . . . . B-126

B3-7 95 Percent Two-Sided Confidence Interval for the Mean Concentration for Solid Segment Sample Data . . . . . . . . . . . . . . . . . . . . . B-129

B3-8 95 Percent Two-Sided Confidence Interval for the Mean Concentration for Supernatant Segment Sample Data . . . . . . . . . . . . . . . . . B-133

B3-9 Analytical-Based Inventory for Composite Sample Data for Tank 241-U-10 . B B-135

B3-10 Analytical-Based Inventory for Solid Segment Sample Data for Tank $241-\mathrm{U}-108 \ldots \ldots \ldots \ldots \ldots \ldots \ldots \ldots$. . . . . . . . . . . . . . . . . . .

B3-11 Analytical-Based Inventory for Liquid Segment Sample Data for Tank 241-U-108

C1-1 95 Percent Confidence Interval Upper Limits for Alpha for Tank 241-U-108

C1-2 95 Percent Confidence Interval Upper Limits for DSC for Tank 241-U-108

C1-3 95 Percent Confidence Interval Lower Limits for Percent Water

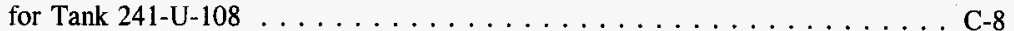

C1-4 95 Percent Confidence Interval Upper Limits for TOC for Tank 241-U-108 . . C-10

D2-1 Sampling and HDW-Based Inventory Estimates for Nonradioactive

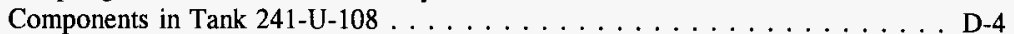

D2-2 Sampling and HDW Model-Based Inventory Estimates for Radioactive Components in Tank $241-\mathrm{U}-108 \ldots \ldots \ldots \ldots \ldots \ldots$. . . . . . . . . . . . .

D3-1 Expected Solids for Tank $241-\mathrm{U}-108 \ldots \ldots \ldots \ldots \ldots \ldots$. . . . . . . . . .

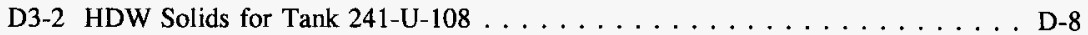




\section{LIST OF TABLES (Continued)}

D3-3 Comparisons of Concentrations in Segments 5, 6, 7, 8 Sample Analysis Average With $\mathrm{S1}$ Saltcake Type . . . . . . . . . . . . . . D-10

D3-4 Inventory Comparisons of Tank Volume Based on Tank 241-U-108 Selected Core Segment Analysis With HDW Model SMMS1 Saltcake Inventory . . . . . D-11

D4-1 Best-Basis Inventory Estimates for Nonradioactive Components in Tank 241-U-108 . . . . . . . . . . . . . . . . . . . . D-14

D4-2 Best-Basis Inventory Estimates for Radioactive Components

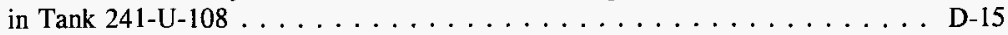




\section{LIST OF TERMS}

\begin{tabular}{|c|c|}
\hline ANOVA & analysis of variance \\
\hline BNW & Battelle Northwest Waste \\
\hline $\mathrm{Btu} / \mathrm{hr}$ & British thermal units per hour \\
\hline CES & consensus exposure standard \\
\hline $\mathrm{Ci}$ & curies \\
\hline $\mathrm{Ci} / \mathrm{L}$ & curies per liter \\
\hline $\mathrm{cm}$ & centimeter \\
\hline $\mathrm{c} / \mathrm{s}$ & counts per second \\
\hline $\mathrm{CW}$ & cladding waste \\
\hline CWR & REDOX cladding waste \\
\hline CWR2 & cladding waste, REDOX, 1961-1972 \\
\hline df & degrees of freedom \\
\hline DQO & data quality objective \\
\hline DSC & differential scanning calorimetry \\
\hline $\mathrm{EB}$ & evaporator bottoms \\
\hline $\mathrm{F}$ & Fahrenheit \\
\hline FIC & Food Instrument Corporation \\
\hline $\mathrm{ft}$ & feet \\
\hline $\mathrm{g} / \mathrm{mL}$ & grams per milliliter \\
\hline g & grams \\
\hline $\mathrm{g} / \mathrm{kg}$ & grams per kilogram \\
\hline $\mathrm{g} / \mathrm{L}$ & grams per liter \\
\hline $\mathrm{GC}$ & gas chromatography \\
\hline GEA & gamma energy analysis \\
\hline HDRL & Hanford defense residual liquor \\
\hline HDW & Hanford defined wastes \\
\hline HTCE & historical tank content estimate \\
\hline IC & ion chromatography \\
\hline ICP & inductively coupled plasma spectroscopy \\
\hline IDLH & immediately dangerous to life and health \\
\hline In. & $\begin{array}{l}\text { Inches } \\
\text { joules per gram }\end{array}$ \\
\hline $\mathrm{kg}^{3}$ & cubic kilograms \\
\hline $\mathrm{kg} / \mathrm{L}$ & kilograms per liter \\
\hline $\mathrm{kg}$ & kilograms \\
\hline kgal & kilogallon \\
\hline $\mathrm{kL}$ & kiloliter \\
\hline $\mathrm{kW}$ & kilowatt \\
\hline L & liter \\
\hline LANL & Los Alamos National Laboratory \\
\hline
\end{tabular}




\section{LIST OF TERMS (Continued)}

LFL lower flammability limit

LL lower limit

LMHC Lockheed Martin Hanford Company

LOW liquid observation well

M molar

m meter

MDL method detection limit

$\mathrm{mg} / \mathrm{kg} \quad$ milligrams per kilogram

$\mathrm{mg} / \mathrm{m}^{3} \quad$ milligrams per cubic meter

$\mathrm{mm} \quad$ millimeter

MTU metric tons uranium

MW metal waste

N N-Reactor waste

n/a not applicable

NCPLX non-complexed waste

$\mathrm{nCi} / \mathrm{g}$ nanocuries per gram

ND not detected

N/D not decided

$\mathrm{n} / \mathrm{T} \quad$ not reported

NIOSH National Institute for Occupational Safety and Health

ORNL Oak Ridge National Laboratory

PHMC Project Hanford Management Contract

PNF partially neutralized waste feed

PNNL Pacific Northwest National Laboratory

ppbv parts per billion by volume

ppm parts per million

ppmv parts per million by volume

QC quality control

REDOX Reduction/Oxidation Plant

REML restricted maximum likelihood

RPD relative percent difference

SACS Surveillance Analysis Computer System

SAP sampling and analysis plan

SMM supernatant mixing model

SMMS1 supernatant mixing model (SMM) wastes from 242-S Evaporator (S1)

SMMS2 supernatant mixing model (SMM) wastes from 242-S Evaporator (S2)

SU supernatant

TBP tributyl phosphate

TCD tank characterization database

TCP tank characterization plan 


\section{LIST OF TERMS (Continued)}

TCR tank characterization report

TGA thermogravimetric analysis

TIC total inorganic carbon

TID tentatively identified compound

TLM tank layer model

TOC total organic carbon

TRU transuranic

TWRS Tank Waste Remediation System

UL upper limit

UR uranium recovery waste

VSS Vapor Sampling System

W watts

WHC Westinghouse Hanford Company

WSTRS Waste Status and Transaction Record Summary

wt\% weight percent

${ }^{\circ} \mathrm{C}$ degrees Celsius

${ }^{\circ} \mathrm{F}$ degrees Fahrenheit

$\mu \mathrm{Ci} / \mathrm{g}$ microcuries per gram

$\mu \mathrm{Ci} / \mathrm{L} \quad$ microcuries per liter

$\mu \mathrm{Ci} / \mathrm{mL}$ microcuries per milliliter

$\mu \mathrm{eq} / \mathrm{g} \quad$ microequivalents per gram

$\mu \mathrm{g} / \mathrm{g} \quad$ micrograms per gram

$\mu \mathrm{g} / \mathrm{mL} \quad$ micrograms per milliliter

$\mu \mathrm{m} \quad$ microns 


\subsection{INTRODUCTION}

One of the major functions of the Tank Waste Remediation System (TWRS) is to characterize wastes in support of waste management and disposal activities at the Hanford Site. Analytical data from sampling and analysis, along with other available information about a tank, are compiled and maintained in a tank characterization report (TCR).

This report and its appendixes serve as the TCR for single-shell tank 241-U-108. The objectives of this report are: 1) to use characterization data in response to technical issues associated with 241-U-108 waste; and 2) to provide a standard characterization of this waste in terms of a best-basis inventory estimate. The response to technical issues is summarized in Section 2.0, and the best-basis inventory estimate is presented in Section 3.0. Recommendations regarding safety status and additional sampling needs are provided in Section 4.0. Supporting data and information are contained in the appendixes. This report also supports the requirements of the Hanford Federal Facility Agreement and Consent Order (Ecology et al. 1996) milestone M-44-10.

\subsection{SCOPE}

Characterization information presented in this report originated from sample analyses and known historical sources. Only the results of recent sampling events will be used to fulfill the requirements of the data quality objectives (DQOs), but other information can be used to support (or question) conclusions derived from these results. Historical information for tank 241-U-108, provided in Appendix A, includes surveillance information, records pertaining to waste transfers and tank operations, and expected tank contents derived from a process knowledge model.

The recent sampling events listed in Table 1-1, as well as sample data obtained prior to 1989, are summarized in Appendix B along with the sampling results. The results of the 1995 and 1996 sampling events satisfied the data requirements specified in the tank characterization plan (TCP) for this tank (Winkelman 1996). The statistical analysis and numerical manipulation of data used in issue resolution are reported in Appendix C.

Appendix D contains the evaluation to establish the best basis for the inventory estimate and the statistical analysis performed for the evaluation. A bibliography that resulted from an in-depth literature search of all known information sources applicable to tank 241-U-108 and its respective waste types is contained in Appendix E. The documents listed in Appendix E may be found in the Lockheed Martin Hanford Corporation Tank Characterization Resource Center. 


\subsection{TANK BACKGROUND}

Tank 241-U-108 is located in the 200 West Area U Tank Farm on the Hanford Site, and is the second tank in a three-tank cascade series. In the mid 1950's, most of the metal waste (MW) was transferred out for uranium recovery. From the second quarter of 1959 until the fourth quarter of 1964, the tank received reduction-oxidation (REDOX) cladding waste. Numerous transfers from the tank took place during the 1960's. In the second quarter of 1964, the tank again received cladding waste from the REDOX plant. From the third quarter of 1968 until the first quarter of 1976, the tank received a combination of N Reactor, REDOX cladding, and evaporator bottoms waste. Some of these wastes were transferred out of tank 241-U-108 during the second quarter of 1972 and the fourth quarter of 1973 . From the second quarter of 1975 through the first quarter of 1977, the tank exchanged evaporator bottoms waste with tank 241-S-102. This was the final major transfer of waste involving tank 241-U-108.

A description of tank 241-U-108 is summarized in Table 1-2. The tank has an operating capacity of $2,010 \mathrm{~kL}(530 \mathrm{kgal})$, and presently contains an estimated $1,771 \mathrm{~kL}(468 \mathrm{kgal})$ of non-complexed waste (Hanlon 1996). The tank is on the Flammable Gas Watch List (Public Law 101-510). 
HNF-SD-WM-ER-639 Rev. 0

Table 1-1. Summary of Recent Sampling.

\begin{tabular}{|c|c|c|c|c|c|}
\hline Sampleynule & Plase & I Localitiol & Segnefitution & Recorerys & 1rass $(g)$ \\
\hline $\begin{array}{l}\text { Core samples } 141 \text {, } \\
145,146 \\
\text { April } 15 \text { to } \\
\text { May } 6,1996\end{array}$ & $\begin{array}{l}\text { Solids and } \\
\text { drainable } \\
\text { liquids }\end{array}$ & $\begin{array}{l}\text { Risers 7,2, } \\
\text { and } 9\end{array}$ & $\begin{array}{l}\text { Half } \\
\text { segments }\end{array}$ & $41-100 \%$ & $\begin{array}{l}\text { See Tables B1-2 } \\
\text { to B1-4 }\end{array}$ \\
\hline $\begin{array}{l}\text { Vapor samples } \\
\text { April } 15 \text { to } \\
\text { May } 8,1996\end{array}$ & Gas & $\begin{array}{l}\text { Tank } \\
\text { headspace, } \\
\text { risers } 7,2, \\
\text { and } 9 ; 6 \mathrm{~m} \\
\text { (19.7 ft) } \\
\text { below top of } \\
\text { riser }\end{array}$ & $\mathrm{n} / \mathrm{a}$ & $\mathrm{n} / \mathrm{a}$ & $n / a$ \\
\hline $\begin{array}{l}\text { Grab samples } \\
\text { U-108-1, U-108-2, } \\
\text { U-108-3 } \\
\text { May 31, } 1995\end{array}$ & Liquids & Riser 7 & None & $100 \%$ & Not determined \\
\hline $\begin{array}{l}\text { Vapor samples } \\
\text { August } 29,1995\end{array}$ & Gas & $\begin{array}{l}\text { Tank } \\
\text { headspace, } \\
\text { riser } 10 \text {; } \\
6.1 \mathrm{~m}(20 \mathrm{ft}) \\
\text { below top of } \\
\text { riser }\end{array}$ & $\mathrm{n} / \mathrm{a}$ & $\mathrm{n} / \mathrm{a}$ & $n / a$ \\
\hline
\end{tabular}

Note:

$\mathrm{n} / \mathrm{a}=$ not applicable 
Table 1-2. Description of Tank 241-U-108.

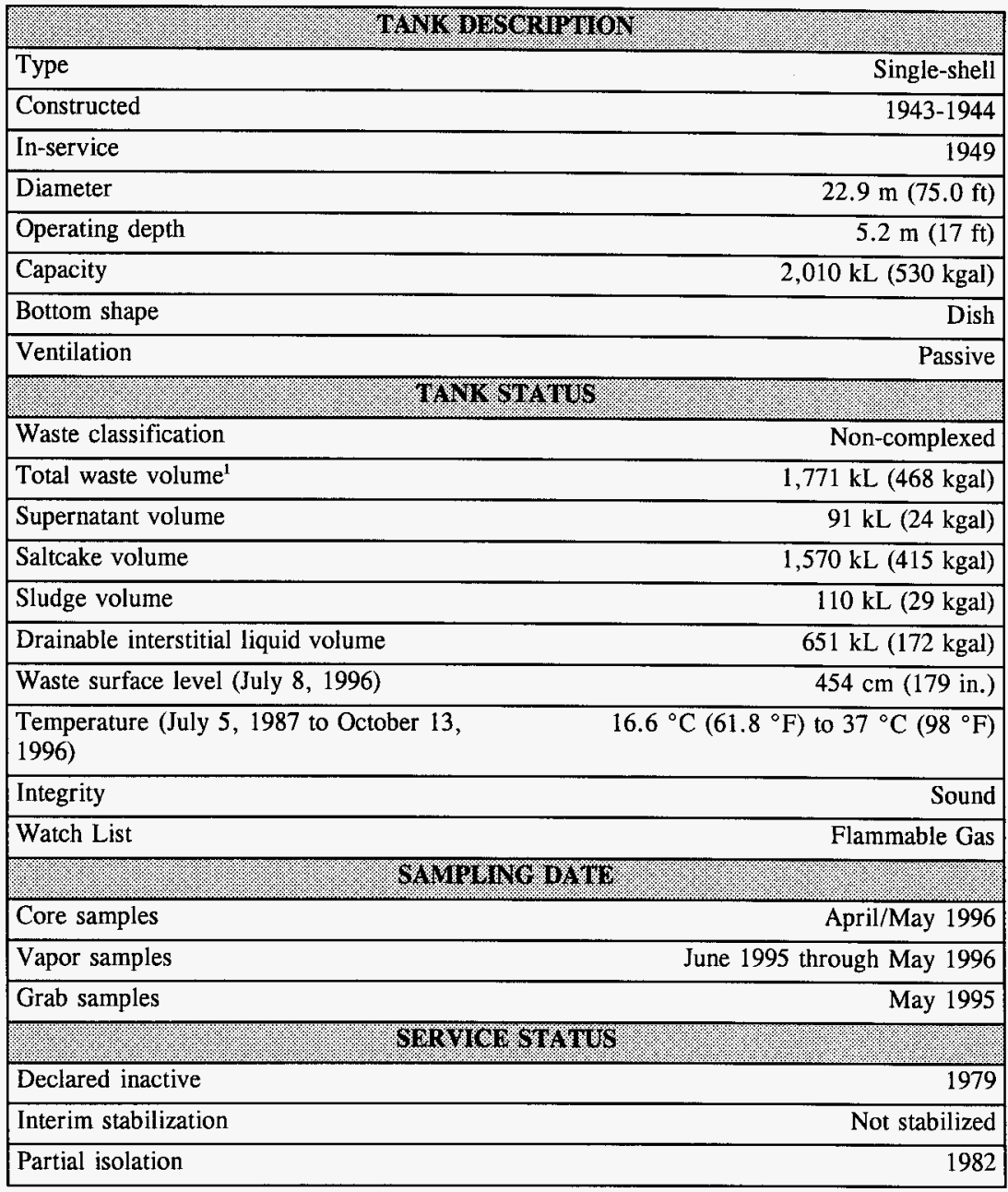

Note:

'Waste volume was estimated from surface-level measurements (Hanlon 1996). 


\subsection{RESPONSE TO TECHNICAL ISSUES}

The following five technical issues have been identified for tank 241-U-108 (Brown et al. 1996). They are:

- Safety screening: Does the waste pose or contribute to any recognized potential safety problems?

- Organic complexants: Does the potential exist for exothermic organic complexant reactions in the waste to produce a radioactive release?

- Vapor screening: 1) Does the tank headspace exceed 25 percent of the lower flammability limit (LFL), and if so, what are the principal fuel components? 2) Is there an organic solvent pool in excess of $1 \mathrm{~m}^{2}\left(10.76 \mathrm{ft}^{2}\right)$ in area that may cause an organic solvent pool fire or ignition of organic solvents entrained in the waste?

- Historical model evaluation: Is the waste inventory generated by a model based on process knowledge and historical information (Agnew et al. 1996a) representative of the current tank waste inventory?

- Compatibility: Do safety or operational problems exist with waste in tank 241-U-108 that could inhibit the transfer of pumpable liquid from the tank into a double-shell receiver tank?

The tank 241-U-108 TCP (Winkelman 1996) provides the types of sampling and analysis used to address the above issues. Data from the recent analysis of three core samples, a grab sample, and tank headspace samples, as well as available historical information, provided the means to respond to these issues. This response is detailed in the following sections. See Appendix B for sample and analysis data for tank 241-U-108.

The 1996 core sampling events took place to satisfy sampling and analysis requirements of the safety screening, organic, and historical DQO documents, and the organic test plan. The 1995 grab samples were taken and analyzed to satisfy the requirements of the compatibility DQO. The 1995 grab samples were taken and analyzed to satisfy the requirements of the generic vapor and rotary core vapor DQOs. The 1996 and 1995 sampling events will be treated separately in the sections below.

The 1995 vapor samples were taken to address the issues listed in the first revision of the tank 241-U-108 TCP (Winkelman 1996). Since the 1995 vapor sampling, the generic vapor and rotary core vapor DQOs have been superseded by the health and safety vapor DQO listed in the latest revision of the tank 241-U-108 TCP (Hewitt 1996). 


\subsection{SAFETY SCREENING AND ORGANIC COMPLEXANT EVALUATION}

The data needed to screen the waste in tank 241-U-108 for potential safety problems are documented in Tank Safety Screening Data Quality Objective (Dukelow et al. 1995). The potential safety problems are exothermic conditions in the waste, flammable gases in the waste and/or tank headspace, and criticality conditions in the waste. The safety screening DQO was not the only safety-related DQO associated with the sampling effort. Tank 241-U-108 is not on the Organic Watch List; however, reviews of waste transfer records indicate that it may contain greater than 3 percent total organic carbon (TOC) on a dry weight basis. The data needed to determine if the waste in tank 241-U-108 poses a potential safety concern with respect to a fuel (organic compounds) and oxidizer (nitrate or nitrite) propagating reaction are documented in Data Quality Objective to Support Resolution of the Organic Complexant Safety Issue (Turner et al. 1995), and Test Plan for Samples From Hanford Waste Tanks 241-BY-103, BY-104, BY-105, BY-106, BY-108, BY-110, TY-103, $U-105, U-107, U-108$, and $U-109$ (Meacham 1995). In addition, organic solvent screening requirements as required in DOE (1996) have been added to all passively ventilated tanks per Cash (1996). Cash (1996) requires that tank 241-U-108 be vapor sampled for total non-methane hydrocarbons as part of the organic DQO (Turner et al. 1995).

\subsubsection{Sampling and Analysis Requirements}

Both the safety screening and organic DQO documents required that two vertical profiles of the waste in tank 241-U-108 be obtained and analyzed at the half-segment level. The upper and lower half subsegments from segment 2 were only obtained from core 146 ; therefore, the sampling requirements of the safety screening and organic DQO documents were not fully met. The analysis requirements of the safety screening DQO were also not fully met, because there was no bulk density determination on the upper half of segment 9 from core 141. Analysis for total non-methane hydrocarbons was performed on the 1995 vapor samples, satisfying the requirement in Cash (1996).

The organic test plan's sampling requirements are not clear. It appears that performing the analyses requested on one sample would meet the sampling requirement of the test plan. Most of the analytical requirements of the test plan were not met, including adiabatic calorimetry and tube propagation tests and analysis for diethylenediamine tetracacetic acid, hydroxyethylenediamine tetraacetic acid, nitrilotriacetic acid, citrate, acetate, formate, and dibutyl phosphate. The missing analyses may be performed on archived samples at a later date. 


\subsubsection{Exothermic Conditions (Energetics)}

The first analytical requirement outlined in the safety screening and organic DQO documents and the organic test plan is to ensure that not enough exothermic constituents (organic compounds, ferrocyanide, or cyanide) are present in tank 241-U-108 to cause a safety hazard. Because of this requirement, energetics in the tank 241-U-108 waste were evaluated using differential scanning calorimetry (DSC). The safety screening and organic DQO documents required that the waste sample profile be tested for energetics every $24 \mathrm{~cm}$ (9.5 in.) to determine if the energetics exceed the DQO notification limit. The threshold for energetics in the DQO documents is $480 \mathrm{~J} / \mathrm{g}$ on a dry-weight basis and $1,200 \mathrm{~J} / \mathrm{g}$ (dry) for the test plan.

Results obtained using DSC indicated that exotherms were apparent in most samples. Analyses were performed on all subsegments and drainable liquids from tank 241-U-108. One sample from the upper half of segment 4 from core 141 had an average DSC result of $496 \mathrm{~J} / \mathrm{g}$ on a dry-weight basis, thus exceeding the DSC notification limit specified in the core sampling and analysis plan (SAP) (Homi 1996). A re-run of a second sample did not exceed the notification limit at $82.6 \mathrm{~J} / \mathrm{g}$ (dry). The mean water content of the sample was 32.8 percent. Two other samples exceeded the DSC notification limit at the one-sided 95 percent upper confidence limit of the mean, although the individual measurements did not exceed the $480 \mathrm{~J} / \mathrm{g}$ notification limit. The one-sided 95 percent lower confidence limits of the means for the respective water content of these two samples were 39.75 and 33.42 percent. Hence, adequate moisture is present to mediate the exothermic reactions.

Waste in tank 241-U-108 was expected to contain organic complexants (Agnew et al. 1996b); therefore, it is reasonable to expect exothermic behavior in the liquid and solid tank waste. The energy equivalent conversion for TOC (based on a sodium acetate average energetics standard) is calculated by converting the analytical results from $\mu \mathrm{g} / \mathrm{g}$ to weight percent (dividing by 10,000). The equation (Meacham 1995) is:

$$
\left[\frac{-1,200 \mathrm{~J} / \mathrm{g}}{4.5 \% \mathrm{TOC}}\right] * \text { measured TOC }(\% \text { dry })=\text { energetics }(\mathrm{J} / \mathrm{g} \text { dry })
$$

The average tank TOC concentration is 0.63 percent (dry). According to the above equation, the TOC would be expected to yield an average exotherm of $168 \mathrm{~J} / \mathrm{g}$ (dry). The actual average of the DSC results was $64.4 \mathrm{~J} / \mathrm{g}$ (dry). The discrepancy may result from the assumption that the TOC is in the form of acetate. Radiolysis and chemical degradation convert higher-energy organic species to lower-energy organic species. The core composite data show that oxalate accounts for 28 percent of the carbon. The amount of TOC present as other low-energy species such as formate was not determined. The TOC measurement may also be low because all the TOC is not being oxidized. However, the data suggest that this is not the case (Section B3.3.5). A third possibility is that some of the TOC has been degraded through chemical or radiological decarboxylation (loss of carbon dioxide and, thus, measured organic carbon). 


\subsubsection{Flammable Gas}

Tank vapor samples, taken in the tank headspace in August 1995 and vacuum pumped from the tank from June 1995 to May 1996, and tank headspace flammability measurements taken prior to and during core sampling in April/May 1996, indicated that little flammable gases were present (highest reading was 6 percent of the LFL). Data from these vapor phase measurements are presented in Appendix B, Section B2.7.

\subsubsection{Criticality}

The safety screening total alpha notification limit is $1 \mathrm{~g} / \mathrm{L}$. However, total alpha is measured in $\mu \mathrm{Ci} / \mathrm{g}$ rather than in $\mathrm{g} / \mathrm{L}$. To convert the notification limit for total alpha into a practical number, it was assumed that all alpha activity originated from Pu-239. Using the average measured tank bulk density of $1.74 \mathrm{~g} / \mathrm{mL}$ (Table B3-7), $1 \mathrm{~g} / \mathrm{L}$ of Pu-239 is equivalent to $35.3 \mu \mathrm{Ci} / \mathrm{g}$ of alpha activity.

Each core subsegment and drainable liquid was analyzed for total alpha activity. The total alpha activity in all core samples was well below the notification limit; the highest activity found at the one-sided 95 percent upper confidence limit of the mean was $0.37 \mu \mathrm{Ci} / \mathrm{g}$. Therefore, no criticality concern exists for tank 241-U-108.

\subsubsection{Total Organic Carbon Content}

Total organic carbon is a primary analyte for the organic DQO, but not for the safety screening DQO. The TOC decision threshold is 3 percent TOC (dry weight). The highest TOC concentration found at the one-sided 95 percent upper confidence limit of the mean was 1.86 percent; thus, no samples exceeded the 3 percent threshold at the 95 percent confidence limit. The sampling requirements were not fully met; otherwise, the tank could be declared "safe" with respect to organic content according to the organic DQO's decision rule.

\subsubsection{Moisture Content}

Moisture content by thermogravimetric analysis (TGA) is another primary analyte for the organic DQO, but not for the safety screening DQO. The percent moisture decision threshold is $\leq 17$ percent. One sample was below this threshold, and 18 samples were below the threshold at the lower 95 percent confidence limit of the mean (Table C1-3). This is not necessarily a safety concern according to the organic DQO, because none of the "dry" subsegments contained greater than 3 percent TOC. The fact that portions of the tank have been shown to be relatively dry is a concern, because the sampling requirements were not met and most samples showed exothermic behavior. 


\subsection{HISTORICAL EVALUATION}

The purpose of the historical evaluation is to determine whether the model based on process knowledge and historical information (Brevick et al. 1996, Agnew et al. 1996b) predicts tank inventories that are in agreement with current tank inventories. If the historical model can be shown to accurately predict the waste characteristics as observed through sample characterization, then there is a possibility that the amount of total sampling and analysis needed may be reduced. Data requirements for this evaluation are documented in Historical Model Evaluation Data Requirements (Simpson and McCain 1995). The historical DQO strives to quantify the errors associated with the tank waste composition predictions based on waste transaction history and waste type compositions. It also identifies key components for each waste type evaluated.

Tank 241-U-108 is classified as "spatially complex" in the historical DQO because historical information indicates that it received many waste types. The analytical results were evaluated against each of the waste types that historical modeling suggests make up the tank waste. The historical estimate of the tank waste is described in Appendix A. Results of the historical model evaluation DQO will be used to quantify the errors associated with the historical tank content estimate (HTCE).

The first step in the historical evaluation is to compare the analytical results with DQO-defined concentration levels for a selected number of analytes. The analytes of interest for each expected waste type are defined in the historical DQO. This comparison may lend strong evidence that the predicted waste type is in the tank and at the predicted location within the waste matrix. The historical DQO requires that two tests be performed for each waste type. The first test determines if the analytes of interest contribute to more than 85 percent of the total waste. The second test checks if the analytical results are $\geq 10$ percent of the DQO levels for each analyte of interest. If a particular waste type passes these two tests, the waste type and layer identification are considered acceptable for further investigation (Simpson and McCain 1995).

Historical modeling estimates that the solid waste in tank 241-U-108 consists of four layers. From the volume of each historical waste layer, the number and identity of the core segments that make up each layer may be identified. Solids from segments 2 through 4 are expected to consist of supernatant mixing model (SMMS2), or S2 saltcake waste type from the 242-S Evaporator. Because of expected horizontal variability in the tank, and to account for possible errors in the waste volume predictions, the lower half of segment 4 was not included in the analysis. Segments 5 through 8 are expected to consist of SMMS1, or S1 saltcake from the 242-S Evaporator. The bottom $15 \mathrm{~cm}(6 \mathrm{in}$.) of waste is expected to be cladding waste generated from 1961 to 1972 (CWR2) from the REDOX facility. The tank layer model indicates that tank 241-U-108 contains $11 \mathrm{~kL}$ ( $3 \mathrm{kgal}$ ) of metal waste (MW). Even if a residual heel is present, it is unlikely that it was sampled, because it would be expected to reside in the tank's dished bottom, which has a volume of $47 \mathrm{~kL}(12.5 \mathrm{kgal})$, and all three core samples were removed from risers along the perimeter of the tank where the dish is inaccessible. Nevertheless, the waste in segment 9 was evaluated for MW. The waste 
recovered from segment 9 varied in color and texture considerably (see Tables B1-2 through B1-4). For this reason, the white material in the upper half of segment 9 from core 141 was treated separately from the black waste from segment 9 from core 145 and the upper half of segment 9 from core 146.

Table 2-1 presents the expected and actual concentrations of the analytes of interest for each waste type expected in tank 241-U-108. The data indicate that the material in segment 2 through the upper half of segment 4 , and in segments 5 through 8 , is consistent with SMMS1 and SMMS2 waste types, respectively, although there are distinct differences in the waste from each volume of the tank: segments 5 through 8 contain more aluminum and carbonate, but much less nitrate, than segments 2 through 4 . The black material from segment 9 was expected to be CWR2 waste; it passes the 10 percent test for CWR2 waste and SMMS2 and SMMS1 waste, but fails the 85 percent mass test. On the other hand, the CWR2 and MW waste type analytes from the historical DQO do not add to 85 percent either. Because of the high sodium and low uranium content in the black segment 9 material, this waste seems to be a mixture of CWR2 and supernatant mixing model (SMM) waste types. The white material from segment 9 also appears to be a mixture of CWR2 and SMM waste types. None of the material from segment 9 is consistent with $\mathrm{MW}$, because it lacks adequate uranium concentrations.

In summary, the waste in tank 241-U-108 appears to be "spatially complex" as labeled in the historical DQO, because four reasonably distinct regions were identified from inspection of the data. The data suggest that the tank contains several layers of waste with differing compositions. However, after comparison with the expected waste types, there is no strong evidence that the saltcake wastes are distinguishable from each other or from the expected waste composition. The sludges observed at the bottom of the tank did not agree with any expected waste composition, and appeared to be a mixture of wastes, but were much different in composition than the saltcake.

\subsection{WASTE COMPATIBILITY EVALUATION}

The compatibility DQO (Carothers 1994) involves two issues: 1) "Assurance that no safety problems are created as a result of commingling wastes under interim storage;" and 2) "Assurance of continued operability during waste transfer and waste concentration/minimization..." In accordance with the compatibility DQO and the waste compatibility SAP (Schreiber 1995), the 1995 grab sample U-108-1 from tank 241-U-108 was analyzed to assess the safety and operational implications of commingling the wastes in the tank and the double-shell tank systems. Safety considerations include criticality, flammable gas generation and accumulation, energetics, corrosion and leakage. Operational considerations include plugged pipelines and equipment, transuranic (TRU) segregation, complexant waste segregation, and heat load limits of the receiving tank. Not all of the safety and operational considerations were within the scope of this report, notably the potential chemical reactivity of the waste in a variety of different situations, and the tendency of the waste to plug piping and equipment. Table 2-2 presents the analyses used to evaluate 


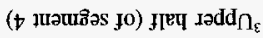

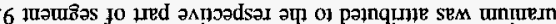

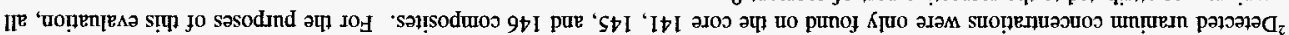

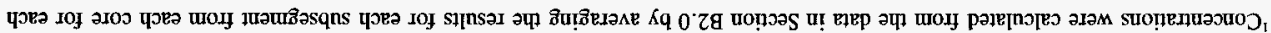

\begin{tabular}{|c|c|c|c|c|c|c|c|c|}
\hline 6.58 & $0^{\circ} \varepsilon L$ & $z \cdot 06$ & $t^{\circ} 00 \mathrm{I}$ & $\mathrm{e} / \mathrm{u}$ & $\mathbf{e} / \mathbf{u}$ & $\mathrm{e} / \mathrm{u}$ & $\mathrm{e} / \mathrm{U}$ & $\begin{array}{l}\text { sseW } \\
\text { fo \% }\end{array}$ \\
\hline$\tau 0+6^{\circ} z$ & $20 E 0^{\circ} t$ & $\mathbf{e} / \mathbf{u}$ & $\mathrm{e} / \mathrm{u}$ & $00 z^{\circ}+9 I$ & $00 S^{\prime} s \mathcal{S}$ & $\mathbf{p} / \mathrm{u}$ & $\mathrm{e} / \mathrm{u}$ & $\Pi$ \\
\hline $0 \angle 6^{\circ} \angle Z$ & $\mathrm{E} / \mathrm{U}$ & $\mathrm{e} / \mathrm{U}$ & $\mathrm{e} / \mathrm{u}$ & $00 S^{\prime} 9 S$ & $\mathbf{B} / \mathbf{U}$ & $\mathbf{e} / \mathrm{u}$ & $\mathrm{e} / \mathrm{u}$ & $\tau_{\tau}^{*} \mathrm{OS}$ \\
\hline $0 S L^{\prime} 8 \varepsilon$ & $\mathbf{e} / \mathbf{U}$ & $\mathrm{e} / \mathrm{u}$ & $\mathrm{e} / \mathrm{u}$ & $00 t^{\circ} 0 E$ & $\mathbf{e} / \mathrm{u}$ & $\mathrm{e} / \mathrm{u}$ & $\mathrm{e} / \mathrm{u}$ & ${ }_{-\varepsilon}^{t} \mathrm{Od}$ \\
\hline$t+8$ & $S \angle 9^{\circ} \varepsilon I$ & $009^{\circ} 0 \mathrm{I}$ & $t+9^{\circ} t$ & $000^{\circ} z \varepsilon$ & $\mathrm{e} / \mathrm{u}$ & $00066 \mathrm{I}$ & $00 I^{\prime}+\tau$ & ${ }_{-\varepsilon}^{\varepsilon} \mathrm{O}$ \\
\hline $000^{\circ} \varepsilon 6 \mathrm{I}$ & $059^{\circ} \angle 6$ & $008^{\circ} 192$ & $00 s^{\circ} t t t$ & $\mathrm{e} / \mathrm{U}$ & $\mathrm{e} / \mathrm{u}$ & $00 L^{\prime} \mathrm{I} 6 \mathrm{I}$ & $00 S^{\prime} 60 z$ &. $\mathrm{EN}^{-}$ \\
\hline$\% \varepsilon^{\prime} \tau \varepsilon$ & $\% 68 \mathcal{E}$ & $\% \varepsilon^{\circ} \varsigma \varepsilon$ & $\% 8.62$ & $\% \mathcal{E}^{\prime} 8 \mathcal{\varepsilon}$ & $\% 2 \cdot I S$ & $\% \tau \angle t$ & $\% 0 \angle Z$ & $\operatorname{IDIE}_{M}$ \\
\hline $000^{\circ} \varepsilon 6 \mathrm{I}$ & $000^{\circ} \angle 0 Z$ & $009^{\circ} \mathrm{SSZ}$ & $00 I^{\prime} E \nabla Z$ & $000^{\circ}+L$ & $000^{\circ} 6 \mathcal{E}$ & $006^{\prime} \mathrm{CDL}$ & $000^{\circ} \angle 6 \mathrm{I}$ & $\mathrm{e}_{\mathrm{N}}$ \\
\hline$\angle 26$ & $29 \varepsilon^{\prime} L$ & $0<z^{6} t$ & $000^{\circ} \varepsilon$ & B/u & $\mathrm{e} / \mathrm{u}$ & $\mathrm{e} / \mathrm{u}$ & $00 \mathcal{E}^{6} \mathrm{I}$ & ID \\
\hline $0 S 68 L$ & $0 \angle 8^{\circ}+I$ & $06 t^{\circ} 9 \mathrm{I}$ & $078^{\circ} 0 \mathrm{I}$ & $\mathrm{e} / \mathrm{u}$ & $00 z^{\prime} s 6$ & $00 \varepsilon^{\prime} \triangleright z$ & $000^{\circ} \nabla t$ & IV \\
\hline$(\mathrm{q} / \mathrm{g})=$ & $(6 / 5 y) \cdot(1)$ & $(078 n)=$. & (1. & (atant) & (8) & (atsing & $(6) 01 \%$ & \multirow[t]{4}{*}{ 2101419 } \\
\hline 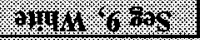 & 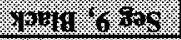 & 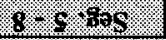 & 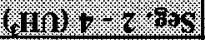 & $\left(1+1 u^{\prime}\right.$ & (3) & Was & 564148 & \\
\hline \multirow{2}{*}{\multicolumn{4}{|c|}{ 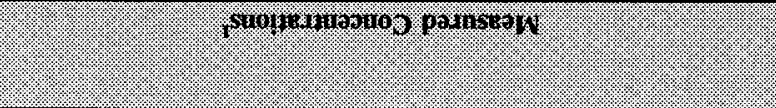 }} & \multicolumn{4}{|c|}{. } & \\
\hline & & & & \multicolumn{4}{|c|}{ 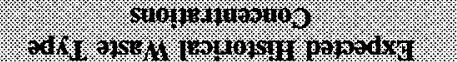 } & \\
\hline
\end{tabular}

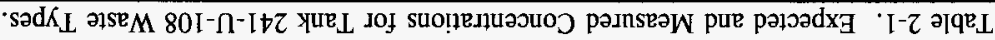


Table 2-2. Decision Variables and Criteria for the Waste Compatibility Data Quality Objective. (2 Sheets)

\begin{tabular}{|c|c|c|c|}
\hline (2. & 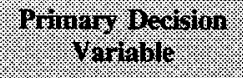 & Q & 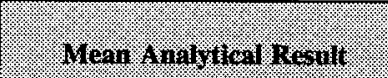 \\
\hline Criticality & {$\left[^{239 / 240} \mathrm{Pu}\right]$} & $\begin{array}{l}{ }^{239 / 240} \mathrm{Pu}+1.077 \mathrm{E}-10 * \\
(\mathrm{U} \text {-gross })>0.013 \mathrm{~g} / \mathrm{L} \\
(>0.7995 \mu \mathrm{Ci} / \mathrm{mL})^{1}\end{array}$ & Less than $9.33 \mathrm{E}-05 \mu \mathrm{Ci} / \mathrm{mL}$ \\
\hline Flammable gas & Specific gravity & $>1.30$ & $1.39^{1}$ \\
\hline Ferrocyanide/organics & Total fuel content & $\begin{array}{l}\text { No exothermic reactions } \\
\text { below } 177^{\circ} \mathrm{C}\left(350^{\circ} \mathrm{F}\right) \text {, absolute } \\
\text { value of exotherm/endotherm } \geq 1 \text {. } \\
\text { No notification required. }\end{array}$ & $\begin{array}{l}\text { No exothermic reactions below } \\
<177^{\circ} \mathrm{C}\left(350^{\circ} \mathrm{F}\right) \text {, absolute } \\
\text { value of exotherm/endotherm } \\
<1 .\end{array}$ \\
\hline Corrosion and leakage & $\begin{array}{l}{\left[\mathrm{NO}_{3}^{-}\right]} \\
{\left[\mathrm{OH}^{-}\right]} \\
{\left[\mathrm{NO}_{2}^{-}\right]}\end{array}$ & $\begin{array}{l}>341,000 \mu \mathrm{g} / \mathrm{mL}^{1} \\
<171 \text { or }>135,000 \mu \mathrm{g} / \mathrm{mL}^{1} \\
<506 \text { or }>252,000 \mu \mathrm{g} / \mathrm{mL}^{1}\end{array}$ & $\begin{array}{l}1.74 \mathrm{E}+05 \mu \mathrm{g} / \mathrm{mL} \\
49,200 \mu \mathrm{g} / \mathrm{mL} \\
1.16 \mathrm{E}+05 \mu \mathrm{g} / \mathrm{mL}\end{array}$ \\
\hline \multicolumn{4}{|l|}{ 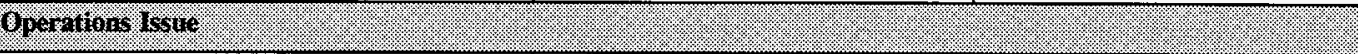 } \\
\hline TRU segregation & TRU elements & $\begin{array}{l}{\left[{ }^{239 / 240} \mathrm{Pu}\right],\left[{ }^{238} \mathrm{Pu}\right],\left[{ }^{241} \mathrm{Am}\right]} \\
{[\mathrm{U} \text { total }],\left[{ }^{243 / 244} \mathrm{Cm}\right],\left[{ }^{237} \mathrm{~Np}\right]} \\
\text { total concentration }>0.1 \mu \mathrm{Ci} / \mathrm{g}\end{array}$ & $\begin{array}{l}{\left[{ }^{239 / 240} \mathrm{Pu}\right]<9.33 \mathrm{E}-05\left[{ }^{241} \mathrm{Am}\right]=} \\
2.39 \mathrm{E}-03 \\
\mathrm{U}, \mathrm{Np} \text {, and } \mathrm{Cm} \text { not analyzed }\end{array}$ \\
\hline Complexant segregation & \multicolumn{3}{|c|}{$\begin{array}{l}\text { Measured by selected analyte concentration, or by performing a boildown test in the } \\
\text { laboratory. }\end{array}$} \\
\hline
\end{tabular}


Table 2-2. Decision Variables and Criteria for the Waste Compatibility Data Quality Objective. (2 Sheets)

\begin{tabular}{|c|c|c|c|}
\hline : Sattry riae & $\begin{array}{l}\text { Prinary Becision } \\
\text { Varable: }\end{array}$ & $\begin{array}{l}\text { Decision Cateria } \\
\text { Thresholal: }\end{array}$ & 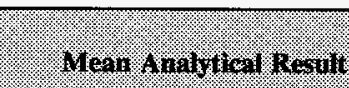 \\
\hline \multicolumn{4}{|l|}{ Oprrations lesire } \\
\hline Heat load & $\begin{array}{l}\text { Heat generation } \\
\text { rate from } \\
\text { radioactive decay }\end{array}$ & $\leq 20,000 \mathrm{~W}(70,000 \mathrm{Btu} / \mathrm{hr})$ & $2,140 \mathrm{~W}$ \\
\hline
\end{tabular}

Note:

'The SAP does not indicate any notification limit, although the DQO does specify the notification limit given. No notifications were made. 
the waste in terms of the safety and operational considerations that are within the scope of this report. The primary decision variable, the notification limit, and the analytical results from the 1995 grab sampling event are listed for each safety or operational issue. All listed analyses and evaluations were well within the notification limits imposed by the waste compatibility DQO.

The analytical mean for ${ }^{239 / 240} \mathrm{Pu}$ was well below the notification limit. Flammable gases may accumulate in wastes with high specific gravity $(>1.30)$. The mean specific gravity for the 1995 grab sample was 1.39. Transfers may be allowed with a specific gravity of the source $>1.3$, provided that the specific gravity of the commingled wastes is $\leq 1.41$. The limit for energetics is an exotherm/endotherm ratio $<1$ for all reactions below $177^{\circ} \mathrm{C}\left(350^{\circ} \mathrm{F}\right)$. This limit was met. The limits for corrosion protection as stated in the compatibility DQO are based on the receiving tank temperature and the concentrations of the corrosion-inhibiting chemicals that are added to the waste. The limits as stated in Table 2-2 apply to tanks with operating temperatures of $<75^{\circ} \mathrm{C}\left(167^{\circ} \mathrm{F}\right)$.

Operations issues are based on the policy of segregating TRU and complexant wastes, avoiding excess heat in the tanks, and ensuring pumpability of the source waste to the receiving tank. The total concentration of TRU elements can be calculated by converting the values to a per-weight basis from the per-volume basis by dividing the analytical result for each radionuclide by the mean drainable liquid specific gravity, and summing the per-weight results. The total can then be compared to the $0.1-\mu \mathrm{Ci} / \mathrm{g}[100-\mathrm{nCi} / \mathrm{g}]$ standard for segregating TRU waste from non-TRU. In the case of tank 241-U-108, all applicable results were less than the detection limit. The waste is non-TRU.

\subsection{TANK VAPOR EVALUATION}

The data needed to determine whether compounds present in the tank headspace were at levels such that the industrial hygiene group needed to be alerted to their presence were documented in Data Quality Objectives for Generic In-Tank Health and Safety Vapor Issue Resolution (Osborne et al. 1994) and Rotary Core Vapor Sampling Data Quality Objective (Price 1994). The sampling and analytical requirements of the two vapor DQOs were specified in the Vapor Sampling and Analysis Plan (Homi 1995). Tank vapors are no longer being addressed as a health concern (Hewitt 1996).

The sampling and analytical requirements of the vapor SAP were only partially met. No vapor samples were analyzed for three of the required organic species, and no vapor sample was sent to Oak Ridge National Laboratory (ORNL) for organic analysis as required in the SAP.

The vapor sampling system, sample device cleaning, sample preparations, and analyses are described in Mahon (1995). The analytical results and interpretation of those results are in Thomas et al. (1996) and are summarized below. 
Of the measured analytes, two exceeded the respective notification limits specified in the vapor SAP: ammonia and N-nitrosodimethylamine. The ammonia concentration (692 ppmv) greatly exceeded the decision threshold of 150 ppmv. An "immediate" notification of the ammonia result was made to the proper personnel (Ligotke 1995). No evidence of a notification of the $\mathrm{N}$-nitrosodimethylamine presence was found. Because the "immediately dangerous to life and health" (IDLH) exposure limit is not available for this compound (NIOSH 1994), and the notification limit is 50 percent of the IDLH value, one could argue that the notification limit was not exceeded. Another concern is that the toxicity decision rule in the vapor DQO (Osborne et al. 1994) requires that carcinogens, teratogens, mutagens, toxins, and irritants be in respective concentrations below one tenth, one half, and one half of the consensus exposure standard (CES). Except for two compounds, the notification limits for organic compounds in the vapor SAP are one half of the IDLH value, which varies greatly from the CES values published (Bratzel 1995) for 43 tanks that were vapor sampled earlier. Neither the SAP nor the analytical report mentions the CES for any analyte.

\subsubsection{Permanent Gases}

The vapor concentrations of the measured permanent gases, $\mathrm{NH}_{3}, \mathrm{NO}_{2}, \mathrm{NO}$, and the vapor mass concentration (primarily $\mathrm{H}_{2} \mathrm{O}$ ), were determined. Two of the four average concentration results for ammonia and water exceeded the minimum of the expected ranges. An immediate notification (phone and electronic memo) was provided on September 9, 1995 after preliminary analytical results indicated the $\mathrm{NH}_{3}$ concentration in tank 241-U-108 exceeded the notification level; notification levels and notification procedures are described in the SAP. The complete results of the permanent-gas analysis of tank $241-\mathrm{U}-108 \mathrm{can}$ be found in Thomas et al. (1996). In summary, hydrogen ( $522 \mathrm{ppmv}$ ) and nitrous oxide (612 ppmv) were observed above the method detection limit in the tank headspace samples, and carbon dioxide in the headspace samples was at a lower concentration than observed in the ambient air.

\subsubsection{Total Non-Methane Hydrocarbons}

The complete results of the total non-methane hydrocarbon analysis of tank 241-U-108 vapor samples can be found in Thomas et al. (1996). In summary, the average concentration in the three tank headspace samples was $11.99 \mathrm{mg} / \mathrm{m}^{3}$. This compares to $6.08 \mathrm{mg} / \mathrm{m}^{3}$ for the sum of all compounds identified in the target and tentatively identified compound (TID) analysis of the SUMMA ${ }^{1}$ canisters.

${ }^{1}$ SUMMA is a trademark of Molectrics, Inc., Cleveland, Ohio. 


\subsubsection{Volatile Organic Analysis}

The complete results of the volatile organic analysis of tank 241-U-108 vapor samples can be found in Thomas et al. (1996). In summary, 14 target analytes above the 5-ppbv reporting cutoff and 12 TIDs above the 10-ppbv reporting cutoff were detected. Thirteen target analytes and all TIDs were identified in two or more samples. The total concentration of the target analytes was found to be $3.47 \mathrm{mg} / \mathrm{m}^{3}$. The total TID concentration was found to be $2.60 \mathrm{mg} / \mathrm{m}^{3}$. The total concentration of all the compounds identified was $6.08 \mathrm{mg} / \mathrm{m}^{3}$. The compound 2-butanone was the only target analyte observed in the upwind ambient air sample. Acetone was observed in the ambient air through the vapor sampling system sample. No TIDs were observed in the two ambient air samples.

\subsubsection{Semi-Volatile Organic Analysis}

The complete results of the sorbent trap analysis of tank $241-\mathrm{U}-108$ vapor samples can be found in Thomas et al. (1996). In summary, 19 target analytes above the 5-ppbv reporting cutoff and 13 TIDs above the 10-ppbv reporting cutoff were detected. Eighteen of 19 target analytes and 9 of 13 TIDs were observed in two or more sorbent traps. Two of 13 TIDs were designated as unknowns. The total concentration of the target analytes was found to be $4.49 \mathrm{mg} / \mathrm{m}^{3}$, the total concentration of the TIDs was found to be $4.25 \mathrm{mg} / \mathrm{m}^{3}$, and the total concentration of all the compounds identified was $8.74 \mathrm{mg} / \mathrm{m}^{3}$.

\subsection{OTHER TECHNICAL ISSUES}

Heat generation and waste temperature are factors used in assessing tank safety. Heat is generated in the tanks from radioactive decay. An estimate of the tank heat load was calculated using the mean Cs-137, Sr-90, and total alpha data from the 1996 core sampling effort. Total alpha was assumed to be all Pu-239. Some of the total alpha is almost certainly due to Am-241; however, this radionuclide produces nearly the same heat per curie as $\mathrm{Pu}-239$. The heat value calculated was $2,140 \mathrm{~W}(7,300 \mathrm{Btu} / \mathrm{hr})$. The HTCE heat load estimate was $3,800 \mathrm{~W}(13,000 \mathrm{Btu} / \mathrm{hr})$. Both these determinations are well below the limit of $11,700 \mathrm{~W}(40,000 \mathrm{Btu} / \mathrm{hr})$ that separates high- and low-heat-load tanks (Smith 1986).

\subsection{SUMMARY}

The analytical results indicate that the tank is conditionally safe. However, the sampling requirements of the safety screening and organic DQO documents were not completely met because two complete vertical profiles were not acquired. Additional sampling may be necessary to satisfy the safety screening and organic DQOs. Three subsamples from tank 241-U-108 exceeded the DSC safety decision threshold limit of $480 \mathrm{~J} / \mathrm{g}$ at the 95 percent upper confidence interval on the mean; however, in each case adequate moisture was present to mediate the exothermic behavior. 
HNF-SD-WM-ER-639 Rev. 0

Nineteen samples were below the organic DQO moisture decision threshold of 17 percent at the lower 95 percent confidence limit on the mean, but the low moisture was mediated by the low TOC content of the samples. A summary of the DQOs and the test plan that applied to this tank is presented in Table 2-3.

Comparison of the waste transfer history and waste historical modeling of the tank with the analytical results suggests the waste does correspond with the predicted waste type compositions, and is "spatially complex" as described in the historical DQO. Also, the presence of MW could not be confirmed because no waste from the tank's dished bottom was sampled. The tank's process history suggests that little MW remains in the tank. The tank's second position in the cascade, and its sluicing history, substantially lower the likelihood of any residual $\mathrm{MW}$. 
Table 2-3. Summary of DQO and Test Plan Evaluations.

\begin{tabular}{|c|c|c|}
\hline WSure & Sub Lisue: & (1) Resull \\
\hline \multirow[t]{3}{*}{$\begin{array}{l}\text { Safety } \\
\text { screening }\end{array}$} & Energetics & $\begin{array}{l}\text { Mean exotherm above } 480 \mathrm{~J} / \mathrm{g} \text { observed in one sample; } \\
\text { two additional samples exceeded } 480 \mathrm{~J} / \mathrm{g} \text { at the } \\
95 \text { percent upper confidence interval on the mean. } \\
\text { Exotherms were mediated by high }(>17 \%) \text { moisture in } \\
\text { each case. }\end{array}$ \\
\hline & Flammable gas & All vapor measurements reported $<25$ percent of LFL. \\
\hline & Criticality & All analyses were well below $35.3 \mu \mathrm{Ci} / \mathrm{g}$ total alpha. \\
\hline \multirow[t]{2}{*}{ Organic } & TOC & $\begin{array}{l}\text { All TOC results were well below the } 3 \% \text { threshold } \\
\text { (dry) at the } 95 \% \text { upper confidence limit of the mean. }\end{array}$ \\
\hline & Moisture & $\begin{array}{l}\text { Nineteen samples were below the moisture decision } \\
\text { threshold of } 17 \% \text { at the lower } 95 \% \text { confidence limit on } \\
\text { the mean. Low moisture was mediated by the low } \\
\text { TOC content of the samples. }\end{array}$ \\
\hline \multirow[t]{2}{*}{ Historical } & $\begin{array}{l}\text { Total mass of } \\
\text { indicators }\end{array}$ & $\begin{array}{l}\text { Failed for MW and CWR2 waste types. Other waste } \\
\text { types passed. }\end{array}$ \\
\hline & $\begin{array}{l}\text { Comparison of } \\
\text { each indicator }\end{array}$ & Passed for SMMS1, SMMS2, and CWR2 waste types. \\
\hline \multirow{4}{*}{$\begin{array}{l}\text { Waste } \\
\text { Compatibility }\end{array}$} & Criticality & Passed \\
\hline & $\begin{array}{l}\text { Flammable gas } \\
\text { retention }\end{array}$ & Failed $^{1}$ \\
\hline & Energetics & Passed \\
\hline & Corrosion & Passed \\
\hline \multirow{4}{*}{$\begin{array}{l}\text { Health and } \\
\text { safety vapor }\end{array}$} & Flammability & Passed \\
\hline & $\begin{array}{l}\text { Carcinogens } \\
\text { Teratogens } \\
\text { Mutagens }\end{array}$ & Passed $^{2}$ \\
\hline & Toxins & Passed \\
\hline & Irritants & $\begin{array}{l}\text { Failed. Ammonia concentration exceeded the } \\
\text { notification limit. }\end{array}$ \\
\hline
\end{tabular}

Notes:

'Flammable gases may accumulate in wastes with high specific gravity $(>1.30)$. The mean specitic gravity for the 1995 grab sample was 1.39 . Transfers may be allowed with a specific gravity of the source $>1.3$, provided that the specific gravity of the commingled wastes is $\leq 1.41$.

${ }^{2}$ Technically, the notification limit for N-nitrosodimethylamine was not exceeded; however, NIOSH (1994) recommends precautions if any amount is detected. 


\subsection{BEST-BASIS INVENTORY ESTIMATE}

Information about the chemical and/or physical properties of tank wastes is used to perform safety analyses, engineering evaluations, and risk assessments associated with waste management activities, as well as to address regulatory issues. Waste management activities include overseeing tank farm operations and identifying, monitoring, and resolving safety issues associated with these operations and with the tank wastes. Disposal activities involve designing equipment, processes, and facilities for retrieving wastes and processing the wastes into a form that is suitable for long-term storage. Chemical inventory information generally is derived using two approaches: 1) component inventories are estimated using the results of sample analyses; and 2) component inventories are predicted using a model based on process knowledge and historical information. The most recent model was developed by Los Alamos National Laboratory (LANL) (Agnew et al. 1996a). Not surprisingly, information derived from these two different approaches is often inconsistent.

An effort is underway to provide waste inventory estimates that will serve as standard characterization information for the various waste management activities (Hodgson and LeClair 1996). As part of this effort, an evaluation of available chemical information for tank 241-U-108 was performed that included:

- Data from recent analyses of three core samples collected in April/May 1996 (Bell 1996). See Appendix B, Section B2.0 for data.

- The solids composite inventory estimate for this tank generated from the Hanford defined waste (HDW) model (Agnew et al. 1996a), developed at Los Alamos National Laboratory.

The results from this evaluation support using the sampling data for tank 241-U-108 for the following reasons.

1. Core sample data were available from three risers at three widely spaced positions. Recovery of sample segments was good for most samples and consistent from core to core. Also, concentrations in each segment were consistent from core to core. Upon inspection of data collection and analysis protocols, no reasons were found to reject the laboratory data.

2. The sample-based inventory reconciles better with the position that the sludge layer in the tank is REDOX cladding waste (CW) rather than bismuth phosphate $M W$, and that sluicing of earlier MW was complete. 
3. The evaporator concentrate wastes (SMMS1 and SMMS2) that make up the majority of the waste volume in tank $241-\mathrm{U}-108$ can be compared with no independent data source. The process of mixing and evaporating supernatants is sufficiently complex that comparison to process flowsheets or multicomponent chemical modeling is impractical.

Best-basis inventory estimates for tank 241-U-108 are presented in Tables 3-1 and 3-2. The projected inventories are based on a sample-derived waste density of $1.74 \mathrm{~g} / \mathrm{mL}$ for segment sample data, $1.71 \mathrm{~g} / \mathrm{mL}$ for core composite data, and $1.40 \mathrm{~g} / \mathrm{mL}$ for the drainable liquid data.

Table 3-1. Best-Basis Inventory Estimates for Nonradioactive Components in Tank 241-U-108. (2 sheets)

\begin{tabular}{|c|c|c|c|}
\hline Whatro & 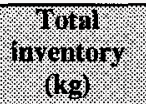 & 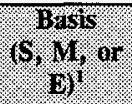 & Sominon \\
\hline $\mathrm{Al}$ & 51,190 & $S$ & Average segment sample data plus liquid data \\
\hline$\overline{\mathrm{Bi}}$ & 437 & $\mathrm{E}$ & Average based on S1 and S2 TCR saltcake data \\
\hline $\mathrm{Ca}$ & 447 & $\mathbf{S}$ & $\begin{array}{l}\text { Average based on acid digest of core composite } \\
\text { data }\end{array}$ \\
\hline $\mathrm{Cl}$ & 10,914 & $S$ & Average segment sample data plus liquid data \\
\hline $\begin{array}{l}\mathrm{TIC} \text { as } \\
\mathrm{CO}_{3}\end{array}$ & 118,500 & $\bar{S}$ & Average segment sample data plus liquid data \\
\hline $\mathrm{Cr}$ & 12,116 & $\mathrm{~S}$ & Average segment sample data plus liquid data \\
\hline $\bar{F}$ & 2,836 & $\mathrm{~S}$ & Average segment sample data plus liquid data \\
\hline $\mathrm{Fe}$ & 507 & $\mathrm{~S}$ & Core composite acid digest samples \\
\hline $\bar{K}$ & 4,800 & $\bar{S}$ & Core composite samples \\
\hline La & $<117$ & $\bar{S}$ & Core composite samples below detection limit \\
\hline$\overline{\mathrm{Mn}}$ & 185 & $\mathrm{~S}$ & Core composite acid digest samples \\
\hline $\mathrm{Na}$ & 726,100 & $\bar{S}$ & Average segment sample data plus liquid data \\
\hline$\overline{\mathrm{Ni}}$ & 150 & $S$ & Core composite acid digest samples \\
\hline $\mathrm{NO}_{2}$ & 157,800 & $\mathbf{S}$ & Average segment sample data plus liquid data \\
\hline $\mathrm{NO}_{3}$ & 879,500 & $\mathbf{S}$ & Average segment sample data plus liquid data \\
\hline $\mathrm{Pb}$ & $<234$ & $S$ & Core composite samples below detection limit \\
\hline $\mathrm{P}$ as $\mathrm{PO}_{4}$ & 50,527 & $\mathrm{~S}$ & Average segment sample data plus liquid data \\
\hline $\mathrm{Si}$ & 830 & $\bar{S}$ & $\begin{array}{l}\text { Average core composite water wash sample } \\
\text { plus liquid }\end{array}$ \\
\hline $\mathrm{S}$ as $\mathrm{SO}_{4}$ & 44,430 & $S$ & Average segment sample data plus liquid data \\
\hline $\mathrm{Sr}$ & $<23.4$ & $\mathrm{~S}$ & Core composite below detection limit \\
\hline TOC & 12,850 & $S$ & Average segment sample data plus liquid data \\
\hline
\end{tabular}


Table 3-1. Best-Basis Inventory Estimates for Nonradioactive Components in Tank 241-U-108. (2 sheets)

\begin{tabular}{|c|c|c|c|}
\hline Analyto & 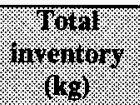 & $(5,14,0$ & Corminent \\
\hline $\mathrm{U}_{\text {TOTAL }}$ & 538 & $\mathrm{~S}$ & $\begin{array}{l}\text { Laser phosphorescence data from core } \\
\text { composite samples }\end{array}$ \\
\hline $\mathrm{Zr}$ & $<23.4$ & $\mathbf{S}$ & Core composite below detection limit \\
\hline
\end{tabular}

Notes:

$$
\begin{array}{ll}
\text { TIC } & =\text { Total inorganic carbon } \\
{ }^{I} \mathrm{~S} & =\text { Sample-based } \\
\mathrm{M} & =\text { HDW model-based } \\
\mathrm{E} & =\text { Engineering assessment-based }
\end{array}
$$

\begin{tabular}{|c|c|c|c|}
\hline Anatite & $\begin{array}{l}\text { Totall } \\
\text { mincmony } \\
(\mathrm{ar})\end{array}$ & (6. M, nists? & fominent \\
\hline${ }^{61} \mathrm{Co}$ & $<61.5$ & $\mathrm{~S}$ & Average solid segment data \\
\hline${ }^{90} \mathrm{Sr}$ & 29,400 & $S$ & Average solid segment data \\
\hline${ }^{137} \mathrm{Cs}$ & 411,000 & $S$ & Average core segment data \\
\hline${ }^{154} \mathrm{Eu}$ & $<241$ & $\bar{S}$ & Average core segment data \\
\hline${ }^{153} \mathrm{Eu}$ & $<884$ & $S$ & Average core segment data \\
\hline${ }^{241} \mathrm{Am}$ & $<1,930$ & $S$ & Average core segment data \\
\hline
\end{tabular}

Table 3-2. Best-Basis Inventory Estimates for Radioactive Components in Tank 241-U-108.

Notes:

$\begin{array}{lll} & \text { IS } & \text { Sample-based } \\ \mathbf{M} & = & \text { HDW model-based } \\ \mathrm{E} & = & \text { Engineering assessment-based }\end{array}$


HNF-SD-WM-ER-639 Rev. 0

This page left blank intentionally. 


\subsection{RECOMMENDATIONS}

Three subsamples from the 1996 core sampling of tank 241-U-108 exceeded the DSC safety decision threshold limit of $480 \mathrm{~J} / \mathrm{g}$ at the 95 percent upper confidence interval on the mean; however, in each case adequate moisture was present to mediate the exothermic behavior. Nineteen samples were below the organic DQO moisture decision threshold of 17 percent at the lower 95 percent confidence limit on the mean, but the low moisture was mediated by the low TOC content of the samples. Despite high DSC and low moisture results on specific samples, the tank would ordinarily be classified as conditionally safe. However, the sampling requirements of the safety screening and organic DQO documents were not completely met.

Two complete vertical profiles of the waste were not acquired and additional sampling may be necessary to satisfy the safety screening and organic DQOs. Obtaining two complete vertical profiles is especially important for this tank because the data from the recent core samples show that: 1) the waste is heterogenous; 2$)$ the waste is relatively dry ( $<17$ percent moisture) in specific locations; 3) that most half-segment subsamples exhibit exothermic behavior; and 4) that three samples were energetic enough to exceed the DSC notification limit at the one-sided 95 percent upper confidence limit on the mean.

The sampling and analysis requirements of the vapor DQO document were not met. Analyses for 1,3-butadiene, butanal, and tributyl phosphate were not performed, possibly because no sample was sent to ORNL for organic analysis. One vapor sample exceeded the notification limit for ammonia. Since the 1995 vapor sampling, tank vapors are no longer being evaluated as a health concern (Hewitt 1996).

The requirements of the historical DQO document were satisfied by the sampling and analysis of the three 1996 core samples. The requirements of the compatibility DQO document were satisfied by the 1995 liquid grab sample. Analysis of the core samples allowed for development of a characterization best-basis inventory.

Table 4-1 summarizes the status of the Project Hanford Management Contract (PHMC) TWRS Program review and acceptance of the sampling and analysis results reported in this tank characterization report. All DQO issues required to be addressed by sampling and analysis are listed in column one of Table 4-1. The second column indicates whether the requirements of the DQO were met by the sampling and analysis activities performed and is answered with "Yes," "No," or "Partially." The third column indicates concurrence and acceptance by the program in TWRS that is responsible for the DQO that the sampling and analysis activities performed adequately meet the needs of the DQO. A "Yes" or "No" in column three indicates acceptance or disapproval of the sampling and analysis information presented in the TCR. Because the waste at the bottom of the tank was not sampled (see Section B3.1) the safety screening DQO has been only partially completed. The upper part of the waste was sampled and analyzed in accordance with the safety screening DQO and accepted by the responsible TWRS program. 
Table 4-2 summarizes the status of PHMC TWRS Program review and acceptance of the evaluations and other characterization information contained in this report. The evaluations specifically outlined in this report are the best-basis inventory evaluation, the historical evaluation, and the evaluation to determine whether the tank is safe, conditionally safe, or unsafe. Column one lists the different evaluations performed in this report. Columns two and three are in the same format as Table 4-1. The manner in which concurrence and acceptance are summarized is also the same as that in Table 4-1. The safety categorization of the tank is listed as "No" in Table 4-2 because the full depth of the waste was not sampled, the number of samples displaying exothermic behavior, and evidence of dry (less than 17 percent moisture) areas in the waste. However, none of the analyses performed on the 1996 core samples indicate any safety problems.

Table 4-1. Acceptance of Tank 241-U-108 Sampling and Analysis.

\begin{tabular}{|c|c|c|}
\hline W. & (4mplingind & 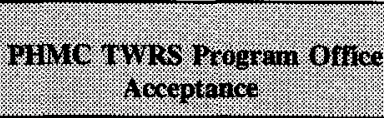 \\
\hline Safety screening DQO & Partially & No \\
\hline Organic complexant DQO & Partially & No \\
\hline Organic solvent screening ${ }^{1}$ & Yes & Yes \\
\hline Historical evaluation DQO & Yes & Yes \\
\hline Waste compatibility DQO & Yes & Yes \\
\hline Generic in-tank health \& safety DQO & Partially & Yes $^{2}$ \\
\hline Organic test plan & Partially & No \\
\hline
\end{tabular}

Notes:

'Considered part of the Organic DQO (Cash 1996)

${ }^{2}$ Tank vapors are no longer being evaluated as a health concern (Hewitt 1996)

Table 4-2. Acceptance of Evaluation of Characterization Data and Information for Tank 241-U-108.

\begin{tabular}{|c|c|c|}
\hline 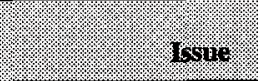 & Womplan & 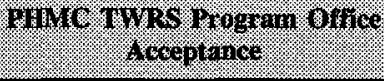 \\
\hline Historical evaluation & Yes & Yes \\
\hline $\begin{array}{l}\text { Safety categorization } \\
\text { (Tank is safe) }\end{array}$ & No & No \\
\hline
\end{tabular}


Resampling of tank 241-U-108 using push-mode core sampling is recommended in order to provide the two full-depth profiles required by the safety screening and organic DQO documents. Further evaluation of the information available on tank 241-U-108 is recommended to determine if additional samples are needed to categorize the tank as "safe." Analysis for total cyanide and adiabatic calorimetry of the samples that exceeded the DSC decision threshold is recommended in order to satisfy the safety screening analytical requirements. In addition, analysis of 1996 core material for adiabatic calorimetry, tube propagation, diethylenediamine tetracacetic acid, hydroxyethylenediamine tetraacetic acid, nitrilotriacetic acid, citrate, acetate, formate, and dibutyl phosphate is recommended to satisfy the analytical requirements of the organic test plan. 
HNF-SD-WM-ER-639 Rev. 0

This page left blank intentionally. 


\subsection{REFERENCES}

Agnew, S. F., J. Boyer, R. A. Corbin, T. B. Duran, J. R. Fitzpatrick, K. A. Jurgensen, T. P. Ortiz, and B. L. Young, 1996a, Hanford Tank Chemical and Radionuclide Inventories: HDW Model Rev. 3, LA-UR-96-858, Los Alamos National Laboratory, Los Alamos, New Mexico.

Agnew, S. F., R. A. Corbin, T. B. Duran, K. A. Jurgensen, T. P. Ortiz, and B. L. Young, 1996b, Waste Status and Transaction Record Summary for the Southwest Quadrant of the Hanford 200 East Area, WHC-SD-WM-TI-614, Rev. 1, Westinghouse Hanford Company, Richland, Washington.

Bell, K. E., 1996, Tank 241-U-108, Cores 141, 145, and 146 Analytical Results for the Final Report, WHC-SD-WM-DP-198, Rev. 0, Westinghouse Hanford Company, Richland, Washington.

Bratzel, D. R., 1995, Headspace Gas and Vapor Characterization Summary for the 43 Vapor Program Suspect Tanks, WHC-SD-WM-ER-514, Rev. 1A, Westinghouse Hanford Company, Richland, Washington.

Brevick, C. H., L. A. Gaddis, and W. W. Pickett, 1996, Historical Tank Content Estimate for the Southwest Quadrant of the Hanford 200 West Areas, WHC-SD-WM-ER-352, Rev. 0B, Westinghouse Hanford Company, Richland, Washington.

Brown, T. M., S. J. Eberlein, J. W. Hunt, and T. J. Kunthara, 1996, Tank Waste Characterization Basis, WHC-SD-WM-TA-164, Rev. 2, Westinghouse Hanford Company, Richland, Washington.

Carothers, 1994, Data Quality Objectives for the Waste Compatibility Program, WHC-SD-WM-DQO-001, Rev. 0, Westinghouse Hanford Company, Richland, Washington.

Cash, R. J., 1996, Scope Increase of 'Data Quality Objective to Support Resolution of the Organic Complexant Safety Issue, 'Rev. 2, (internal memorandum 79300-96-029 to S. J. Eberlein, July 12), Westinghouse Hanford Company, Richland, Washington.

DOE, 1996, Recommendation 93-5 Implementation Plan, DOE/RL-94-0001, Rev. 1, U. S. Department of Energy, Richland Field Office, Richland, Washington.

Dukelow, G. T., J. W. Hunt, H. Babad, and J. E. Meacham, 1995, Tank Safety Screening Data Quality Objective, WHC-SD-WM-SP-004, Rev. 2, Westinghouse Hanford Company, Richland, Washington. 
Ecology, EPA, and DOE, 1996, Hanford Federal Facility Agreement and Consent Order, as amended, Washington State Department of Ecology, U.S. Environmental Protection Agency, and U.S. Department of Energy, Olympia, Washington.

Hanlon, B. M., 1996, Waste Tank Summary Report for Month Ending September 30, 1996, WHC-EP-0182-102, Westinghouse Hanford Company, Richland, Washington.

Hewitt, E. R., 1996, Tank Waste Remediation System Resolution of Potentially Hazardous Vapor Issues, WHC-SD-TWR-RPT-001, Rev. 0, Westinghouse Hanford Company, Richland, Washington.

Homi, C. S., 1995, Vapor Sampling and Analysis Plan, WHC-SD-WM-TP-335, Rev. OF, Westinghouse Hanford Company, Richland, Washington.

Homi, C. S., 1996, Tank 241-U-108 Push Mode Core Sampling and Analysis Plan, WHC-SD-WM-TSAP-049, Rev. 0, Westinghouse Hanford Company, Richland, Washington.

Hodgson, K. M., and M. D. LeClair, 1996, Work Plan for Defining a Standard Inventory Estimate for Wastes Stored in Hanford Site Underground Tanks, WHC-SD-WM-WP-311, Rev. 1, Westinghouse Hanford Company, Richland, Washington.

Ligotke, M. W., 1995, Tank U-108: NH3 Exceeds Limit, (electronic mail to D. R. Bratzel and D. R. Carls, September 9), Pacific Northwest National Laboratory, Richland, Washington.

Mahon, R. D., 1995, Vapor and Gas Sampling of Single-Shell Tank.241-U-108 Using the Vapor Sampling System, WHC-SD-WM-RPT-180, Rev. 1, Westinghouse Hanford Company, Richland, Washington.

Meacham, J. E., 1995, Test Plan for Samples From Hanford Waste Tanks 241-BY-103, $B Y-104, B Y-105, B Y-106, B Y-108, B Y-110, T Y-103, U-105, U-107, U-108$, and U-109, WHC-SD-WM-TP-378, Rev. 0, Westinghouse Hanford Company, Richland, Washington.

NIOSH 1994, NIOSH Pocket Guide to Chemical Hazards, Publication Number 94-116, U. S. Government Printing Office, Washington, DC, 20402.

Osborne, J. W., J. L. Huckaby, T. P. Rudolph, E. R. Hewitt, D. D. Mahlum, J. Y. Young, and C. M. Anderson, 1994, Data Quality Objectives for Generic In-Tank Health and Safety Issue Resolution, WHC-SD-WM-DQO-002, Rev. 0, Westinghouse Hanford Company, Richland, Washington. 
Price, O. N., 1994, Rotary Core Vapor Sampling Data Quality Objectives, WHC-S-WM-SP-003, Rev. 0, Westinghouse Hanford Company, Richland, Washington.

Public Law 101-510, 1990, "Safety Measures for Waste Tanks at Hanford Nuclear Reservation," Section 3137 of National Defense Authorization Act for Fiscal Year 1991.

Schreiber, R. D., 1995, Compatibility Grab Sampling and Analysis Plan, WHC-SD-WM-TP-330, Rev. 0, Westinghouse Hanford Company, Richland, Washington.

Simpson, B. C., and D. J. McCain, 1995, Historical Model Evaluation Data Requirements, WHC-SD-WM-DQO-018, Rev. 0A, Westinghouse Hanford Company, Richland, Washington.

Smith, D. A., 1986, Single-Shell Tank Isolation Safety Analysis Report, WHC-SD-WM-SAR-006, Rev. 2, Westinghouse Hanford Company, Richland, Washington.

Turner, D. A., H. Babad, L. L. Buckley, and J. E. Meacham, 1995, Data Quality Objective to Support Resolution of the Organic Complexant Safety Issue, WHC-SD-WM-DQO-006, Rev. 2, Westinghouse Hanford Company, Richland, Washington.

Thomas, B. L., T. W. Clauss, J. C. Evana, B. D. McVeety, K. H. Pool, K. B. Olsen, J. S. Fruchter, and M. W. Ligotke, 1996, Headspace Vapor Characterization of Hanford Waste Tank 241-U-108: Results from Samples Collected on 08/29/95, PNNL-10961, Pacific Northwest National Laboratory, Richland, Washington.

Winkelman, W. D., 1996, Tank 241-U-108 Tank Characterization Plan, WHC-SD-WM-TP-315, Rev. 3, Westinghouse Hanford Company, Richland, Washington. 
HNF-SD-WM-ER-639 Rev. 0

This page left blank intentionally. 
WHC-SD-WM-ER-639 Rev. 0

\author{
APPENDIX A \\ HISTORICAL TANK INFORMATION
}


WHC-SD-WM-ER-639 Rev. 0

This page left blank intentionally. 


\section{APPENDIX A}

\section{HISTORICAL TANK INFORMATION}

Appendix A describes tank 241-U-108 based on historical information. For this report, historical information includes any information about the fill history, waste types, surveillance, or modeling data about the tank. This information is necessary for providing a balanced assessment of the sampling and analytical results.

This appendix contains the following information:

- Section A1: Current status of the tank, including the current waste levels as well as the stabilization and isolation status of the tank.

- Section A2: Information about the design of the tank.

- Section A3: Process knowledge of the tank; that is, the waste transfer history and the estimated contents of the tank based on modeling data.

- Section A4: Surveillance data for tank 241-U-108, including surface-level readings, temperatures, and a description of the waste surface based on photographs.

- Section A5: References for Appendix A.

Historical sampling results (results from samples obtained prior to 1989) are included in Appendix B.

\section{A1.0 CURRENT TANK STATUS}

As of July 31 , 1996, tank $241-\mathrm{U}-108$ contained an estimated $1,771 \mathrm{~kL}$ (468 kgal) of waste classified as non-complexed (Hanlon 1996). Liquid waste volumes are estimated using a level measurement gauge. The solid waste volumes are estimated using a sludge level measurement device. The solid waste volume was last updated on June 30, 1996. The amounts of various waste phases in the tank are presented in Table A1-1.

Tank 241-U-108 is out of service, as are all single-shell tanks. It is partially interim isolated, is categorized as sound, and has not been interim stabilized. The tank is on the Hydrogen/Flammable Gas Watch List and is passively ventilated. All monitoring systems were in compliance with documented standards as of September 30, 1996 (Hanlon 1996). 
Table A1-1. Tank Contents Status Summary.

\begin{tabular}{|c|c|}
\hline 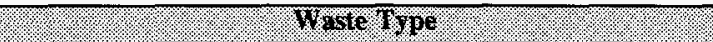 & 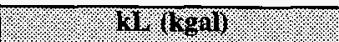 \\
\hline Total waste & $1,771(468)$ \\
\hline Supernatant liquid & $91(24)$ \\
\hline Sludge & $110(29)$ \\
\hline Saltcake & $1,570(415)$ \\
\hline Drainable interstitial liquid & $651(172)$ \\
\hline Drainable liquid remaining & $742(196)$ \\
\hline Pumpable liquid remaining & 791 (209) \\
\hline
\end{tabular}

Note:

Hanlon (1996)

\section{A2.0 TANK DESIGN AND BACKGROUND}

The 241-U Tank Farm was constructed during 1943 and 1944 in the 200 West Area. It is one of twelve 2,010-kL (530-kgal) tanks in U Farm. Built according to the first generation design, the 241-U Tank Farm was designed for nonboiling waste with a maximum fluid temperature of $104^{\circ} \mathrm{C}\left(220^{\circ} \mathrm{F}\right)$. A cascade overflow line $75 \mathrm{~mm}(3 \mathrm{in}$.$) in diameter$ connects tank 241-U-108 as second in a cascade series of three tanks beginning with tank 241-U-107 and finishing with tank 241-U-109. Each tank in the cascade series is set $30 \mathrm{~cm}$ $(1 \mathrm{ft})$ lower in elevation than the preceding tank.

Tank 241-U-108 has a dished bottom with a 1.2-m (4-ft) radius knuckle, a diameter of $23 \mathrm{~m}$

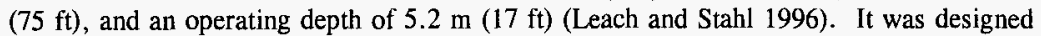
with a primary mild steel liner (ASTM A283 Grade C) and a concrete dome with 13 risers. The tank is set on a reinforced concrete foundation. The tank and foundation were waterproofed with a coating of tar covered by a three-ply, asphalt-impregnated waterproofing fabric. The waterproofing was protected by a welded-wire-reinforced mixture of cement, sand, and water. Two coats of primer were sprayed on all exposed interior tank surfaces (Rogers and Daniels 1944). The tank ceiling dome was covered with three applications of magnesium zinc fluorosilicate wash. Lead flashing was used to protect the joint where the steel liner meets the concrete dome. Asbestos gaskets were used to seal the risers in the tank dome.

A plan view depicting riser configuration is shown as Figure A2-1. Risers 9 and 10 , $100 \mathrm{~mm}$ (4 in.) in diameter, and 2 and 7, $300 \mathrm{~mm}$ (12 in.) in diameter, are available for sampling (Lipnicki 1996). Table A2-1 shows numbers, diameters, and descriptions of the risers and the nozzles. The tank's 13 risers range in diameter from $100 \mathrm{~mm}$ (4 in.) to $1.1 \mathrm{~m}$ (42 in.). A tank cross-section showing the approximate waste level and a schematic of the tank equipment is shown in Figure A2-2. 
WHC-SD-WM-ER-639 Rev. 0

Figure A2-1. Riser Configuration for Tank 241-U-108.

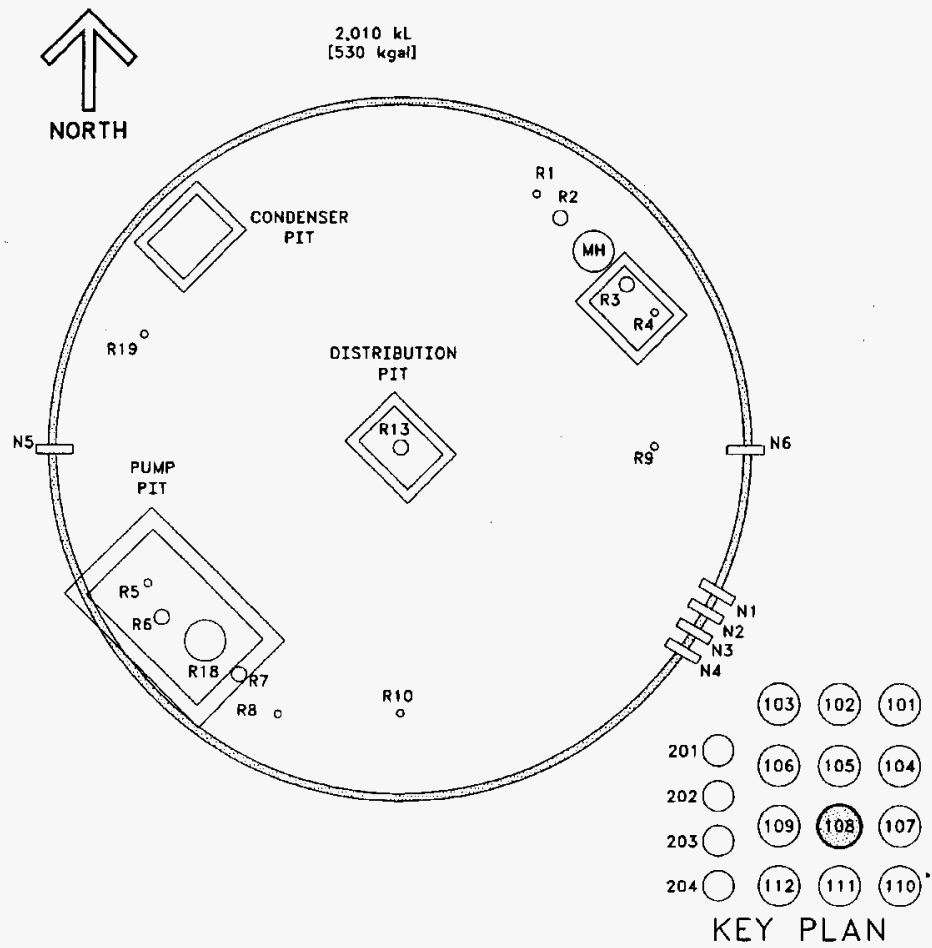


Table A2-1. Tank 241-U-108 Risers. ${ }^{1,2}$

\begin{tabular}{|c|c|c|}
\hline Nimor & QWineter & 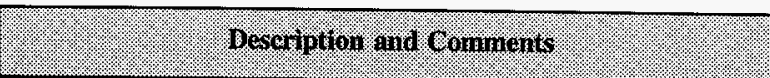 \\
\hline R1 & 4 & $\begin{array}{l}\text { Thermocouple tree [Benchmark CEO-37531, December 11, } \\
\text { 1986] }\end{array}$ \\
\hline $\mathrm{R} 2$ & 12 & Blind flange \\
\hline$\overline{\mathrm{R} 3}$ & 12 & Sluice nozzle, weather covered \\
\hline$\overline{\mathrm{R} 4}$ & 4 & Recirculation line dip legs, weather covered \\
\hline R5 & 4 & Recirculation line dip legs, weather covered \\
\hline R6 & 12 & Sluice nozzle, weather covered \\
\hline$\overline{\mathrm{R} 7}$ & 12 & B-222 observation port \\
\hline R8 & 4 & $\begin{array}{l}\text { ENRAF }^{3} 854, \text { ECN-620751, February 27, } 1995 \text { [prior Food } \\
\text { Instrument Corporation (FIC) gauge] }\end{array}$ \\
\hline R9 & 4 & $\begin{array}{l}\text { Sludge measurement port [Benchmark CEO-37531, December 11, } \\
\text { 1986] }\end{array}$ \\
\hline R10 & 4 & $\begin{array}{l}\text { Breather filter [Standard Hydrogen Monitor System/breather } \\
\text { filter, ECN-W369-021, January 13, 1995] }\end{array}$ \\
\hline R13 & 12 & Distributor Jet for saltwell pumping \\
\hline R18 & $\overline{42}$ & Sludge pump, weather covered \\
\hline R19 & 4 & B-436 liquid observation well (LOW) \\
\hline N1 & 3 & Spare, capped \\
\hline $\mathrm{N} 2$ & 3 & Spare, capped \\
\hline N3 & 3 & Spare, capped \\
\hline N4 & 3 & Spare, capped \\
\hline N5 & 3 & Outlet overflow \\
\hline N6 & 3 & Inlet overflow \\
\hline
\end{tabular}

Notes:

$\mathrm{CEO}=$ Change engineering order
$\mathrm{ECN}=$ Engineering change notice

'Alstad 1993

${ }^{2}$ Tran 1993

${ }^{3}$ Trademark of ENRAF Corporation, Houston, Texas. 
Figure A2-2. Tank 241-U-108 Cross Section and Schematic.

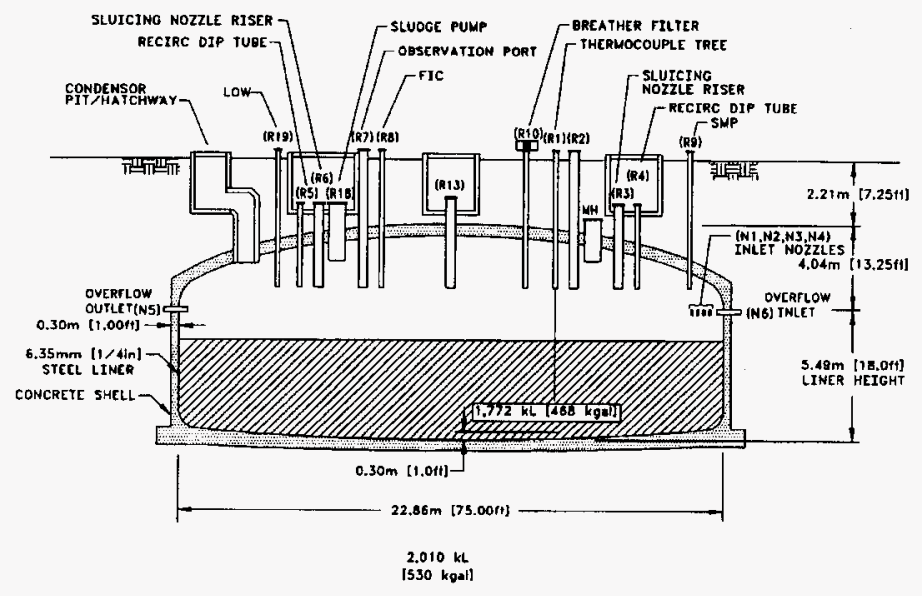




\section{A3.0 PROCESS KNOWLEDGE}

The sections below: 1) provide information about the waste transfer history of tank $241-U-108$; 2) describe the process wastes that were transferred; and 3) give an estimate of current tank contents based on waste transfer history.

\section{A3.1 WASTE TRANSFER HISTORY}

Table A3-1 summarizes the major ( $>1,000 \mathrm{~kL}$ ) waste transfers to and from tank 241-U-108 (Agnew et al. 1996a). Tank 241-U-108 began receiving MW in the first quarter of 1949 via the cascade line from tank 241-U-107. The tank was full and cascading to tank 241-U-109 by the end of the first quarter, and the cascade continued until the fourth quarter of 1954 . The tank received flush water in the third quarter of 1953 and in the third and fourth quarters of 1954. In the fourth quarter of 1955, tank 241-U-108 again received flush water. Some of this waste was sluiced to tank 241-U-109. Most of the waste from the tank 241-U-108 was transferred out for the uranium recovery process by the first quarter of 1956.

From the second quarter of 1959 until the fourth quarter of 1964, tank 241-U-108 received REDOX cladding waste from tank 241-S-107. During the fourth quarter of 1961, the tank sent waste to tank 241-U-105. During the second quarter of 1963 , the tank sent waste to tank 241-T-101. From the second through fourth quarters of 1968 , the tank sent waste to tank 241-TX-118. In the second quarter of 1964 and the third quarter of 1968, the tank received cladding waste from the REDOX plant. The tank received supernatant waste from tank 241-U-107 from the fourth quarter of 1972 until the first quarter of 1976. During the second quarter of 1972 and the fourth quarter of 1973, the tank sent waste to tanks 241-TX-101 and 241-S-101, respectively. From the second quarter of 1975 through the first quarter of 1977, the tank received evaporator bottom waste from tank 241-S-102. During the same time period, supernatant waste was sent to tank 241-U-111, and evaporator feed waste was sent to $241-\mathrm{S}-102$.

\section{A3.2 HISTORICAL ESTIMATION OF TANK CONTENTS}

The historical transfer data used for this estimate are from the following sources.

- Waste Status and Transaction Record Summary for the Northwest Quadrant of the Hanford 200 West Area (WSTRS) (Agnew et al. 1996b). The WSTRS is a tankby-tank quarterly summary spreadsheet of waste transactions.

- Hanford Tank Chemical and Radionuclide Inventories: HDW Model Rev. 3 (Agnew et al. 1996a). This document contains the Hanford defined waste (HDW) list, the supernatant mixing model (SMM), and the tank layer model (TLM). 
Table A3-1. Tank 241-U-108 Major Transfers. ${ }^{1,2}$

\begin{tabular}{|c|c|c|c|c|c|}
\hline \multirow{2}{*}{ 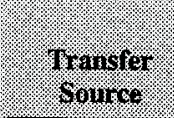 } & \multirow{2}{*}{$\begin{array}{l}\text { Manster } \\
\text { Tistination }\end{array}$} & \multirow[b]{2}{*}{ Whiste Type } & \multirow[b]{2}{*}{ Time Period } & \multicolumn{2}{|c|}{$\begin{array}{l}\text { Estimated Wiste } \\
\text { Volunis. }\end{array}$} \\
\hline & & & & 11: & kga \\
\hline \multirow[t]{2}{*}{$241-U-107$} & & MW & $1949-1954$ & 8097 & 2139 \\
\hline & 241-U-109 & MW & $1949-1955$ & -7908 & -2089 \\
\hline \multirow[t]{2}{*}{ Miscellaneous } & & flush water & $1953-1955$ & 4550 & 1202 \\
\hline & 241-U-109 & MW & 1955 & -2006 & -530 \\
\hline \multirow[t]{3}{*}{ 241-S-107 } & & $\overline{C W R}$ & $1959-1964$ & 4039 & 1067 \\
\hline & $241-U-105$ & CWR & 1961 & -1220 & -322 \\
\hline & $241-T-101$ & CWR & 1963 & -1640 & -432 \\
\hline \multirow[t]{2}{*}{ REDOX Plant } & & CWR & 1964,1968 & 3590 & 948 \\
\hline & 241-TX-118 & CWR & 1968 & -7366 & -1946 \\
\hline \multirow[t]{4}{*}{ 241-U-107 } & & SU & $1968-1972$ & 5913 & 1562 \\
\hline & 241-TX-101 & SU & 1972 & -1660 & -439 \\
\hline & 241-S-101 & SU & 1973 & -1270 & -335 \\
\hline & 241-U-111 & $\mathrm{SU}$ & 1975 & -3748 & -990 \\
\hline 241-U-107 & & $\mathrm{SU}$ & $1975-1976$ & 5618 & 1484 \\
\hline \multirow[t]{2}{*}{$241-S-102$} & & EB & $1975-1977$ & 1242 & 328 \\
\hline & $241-S-102$ & EB & $1975-1977$ & -1749 & -462 \\
\hline
\end{tabular}

Notes:

$\begin{array}{lll}\text { CWR } & = & \text { REDOX cladding waste } \\ \text { EB } & = & \text { Evaporator bottoms } \\ \text { SU } & = & \text { Supernatant }\end{array}$

'Agnew et al. 1996a

${ }^{2}$ Because only major transfers are listed, the sum of these transfers will not equal the current tank waste volume. 
- Historical Tank Content Estimate for the [Northeast, Northwest, Southeast, or Southwest] Quadrant of the Hanford 200 [East or West] Area. This set of four documents compiles and summarizes much of the process history, design, and technical information regarding the underground waste storage tanks in the 200 Areas.

- Tank Layer Model. The TLM defines the sludge and saltcake layers in each tank using waste composition and waste transfer information.

- Supernatant Mixing Model. This is a subroutine with the HDW model that calculates the volume and composition of certain supernatant blends and concentrates.

Using these records, the TLM defines the sludge and saltcake layers in each tank. The SMM uses information from both WSTRS and the TLM to describe the supernatants and concentrates in each tank. Together, the WSTRS, TLM, and SMM determine each tank's inventory estimate. These model predictions are considered estimates that require further evaluation using analytical data.

Based on the TLM and the SMM, tank 241-U-108 contains 4 layers of waste, not including the supernatant, listed from last deposit into the tank to the first deposit; $697 \mathrm{~kL}$ (184 kgal) of 242-S Evaporator salt slurry (SMMS2), $874 \mathrm{~kL}$ (231 kgal) of 242-S Evaporator saltcake (SMMS1), $98 \mathrm{~kL}$ (26 kgal) of CWR2, and $11 \mathrm{~kL}$ (3 kgal) of metal waste (MW).

A graphical representation of the estimated waste types and volumes for these layers can be seen in Figure A3-1.

The SMMS1 and SMMS2 waste compositions are calculated by the SMM on an individual tank basis and are considered concentrated supernatants. The MW layer should contain, from highest concentration above one weight percent, the following constituents: uranium, hydroxide, sodium, carbonate, and phosphate. Constituents contained in this layer above a tenth of a weight percent are sulfate, iron, nitrate, and calcium. The MW should have a relatively small activity because of the small quantities of cesium-137 $\left({ }^{137} \mathrm{Cs}\right)$ and strontium-90 $\left({ }^{90} \mathrm{Sr}\right)$. The REDOX cladding waste layer should contain, from highest concentration above one weight percent, the following constituents: hydroxide, aluminum, uranjum, lead, nitrate, sodium, iron, and carbonate. Constituents contained in this layer above a tenth of a weight percent are calcium, nitrite, and mercury. REDOX cladding waste should have a relatively small activity due to the small quantities of ${ }^{137} \mathrm{Cs}$ and ${ }^{90} \mathrm{Sr}$.

Table A3-2 shows the historical estimate of the expected waste constituents and their concentrations. 
Figure A3-1. Tank Layer Model.

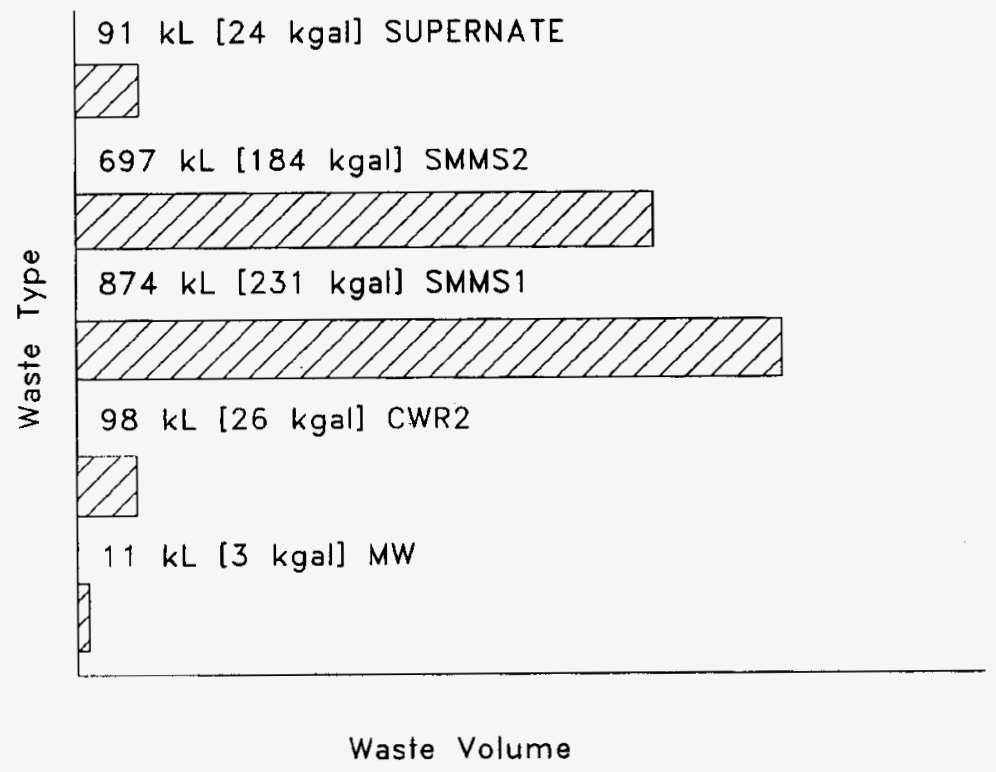


Table A3-2. Historical Tank Inventory Estimate. ${ }^{1,2}$ (2 sheets)

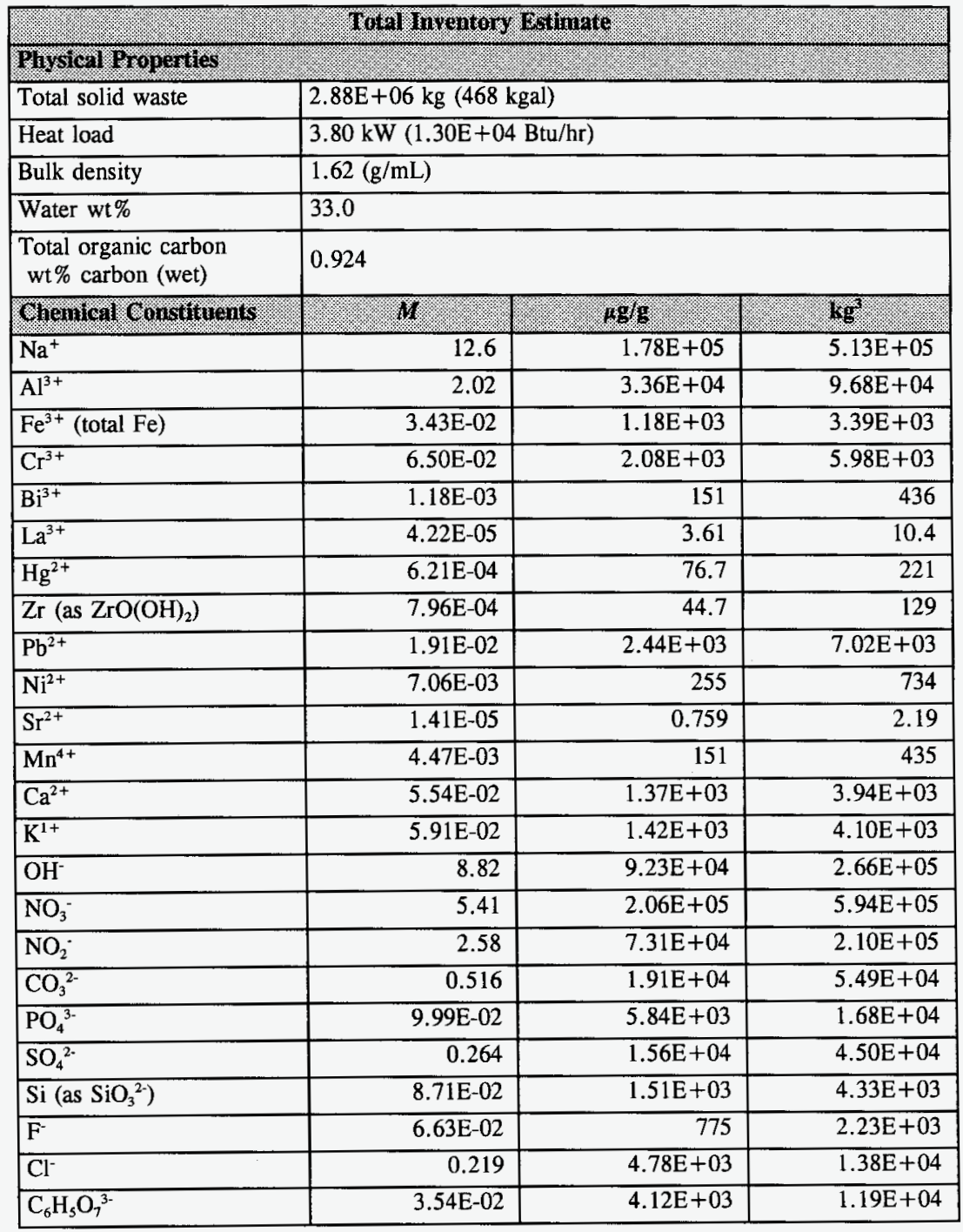


Table A3-2. Historical Tank Inventory Estimate. ${ }^{1,2}$ (2 sheets)

\begin{tabular}{|c|c|c|c|}
\hline 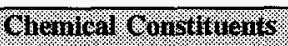 & YY. & poni. & 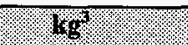 \\
\hline EDTA $^{4}$ & $1.86 \mathrm{E}-02$ & $3.29 \mathrm{E}+03$ & $9.47 \mathrm{E}+03$ \\
\hline HEDTA $^{3-}$ & $3.41 \mathrm{E}-02$ & $5.76 \mathrm{E}+03$ & $1.66 \mathrm{E}+04$ \\
\hline glycolate & 0.110 & $5.08 \mathrm{E}+03$ & $1.46 \mathrm{E}+04$ \\
\hline acetate & $9.55 \mathrm{E}-03$ & 347 & 998 \\
\hline oxalate $^{2-}$ & $3.61 \mathrm{E}-05$ & 1.96 & 5.63 \\
\hline DBP & $2.25 \mathrm{E}-02$ & $3.69 \mathrm{E}+03$ & $1.06 \mathrm{E}+04$ \\
\hline Butanol & $2.25 \mathrm{E}-02$ & $1.03 \mathrm{E}+03$ & $2.95 \mathrm{E}+03$ \\
\hline $\mathrm{NH}_{3}$ & $5.96 \mathrm{E}-02$ & 623 & $1.79 \mathrm{E}+03$ \\
\hline $\mathrm{Fe}(\mathrm{CN})_{6}^{4-}$ & 0 & 0 & 0 \\
\hline \multicolumn{4}{|l|}{ Rodiological ogrstinents } \\
\hline $\mathrm{Pu}$ & & $0.229(\mu \mathrm{Ci} / \mathrm{g})$ & $11.0(\mathrm{~kg})$ \\
\hline $\mathrm{U}$ & $5.30 \mathrm{E}-02(\mathrm{M})$ & $7.77 \mathrm{E}+03(\mu \mathrm{g} / \mathrm{g})$ & $2.24 \mathrm{E}+04(\mathrm{~kg})$ \\
\hline Cs & $0.261(\mathrm{Ci} / \mathrm{L})$ & $161(\mu \mathrm{Ci} / \mathrm{g})$ & $4.63 \mathrm{E}+05(\mathrm{Ci})$ \\
\hline $\mathbf{S r}$ & $0.137(\mathrm{Ci} / \mathrm{L})$ & $84.3(\mu \mathrm{Ci} / \mathrm{g})$ & $2.43 \mathrm{E}+05(\mathrm{Ci})$ \\
\hline
\end{tabular}

Notes:

DBP = Dibutyl phosphate

${ }^{1}$ Agnew et al. $1996 \mathrm{~b}$

${ }^{2}$ These predictions have not been validated and should be used with caution.

${ }^{3}$ Differences exist among the inventories in this column and the inventories calculated from the two sets of concentrations. 


\section{A4.0 SURVEILLANCE DATA}

Tank 241-U-108 surveillance includes surface-level measurements (liquid and solid) and temperature monitoring inside the tank (waste and headspace). The data provide the basis for determining tank integrity.

Liquid-level measurement may indicate if a tank has a major leak. Solid surface-level measurements provide an indication of physical changes and consistency of the solid layers. Dry wells located around the tank perimeter may show increased radioactivity resulting from leaks.

\section{A4.1 SURFACE-LEVEL READINGS}

Tank 241-U-108 has a liquid observation well (LOW) located in riser 19. A gauge was used to monitor the waste surface level in tank $241-\mathrm{U}-108$ through riser 8 in the automatic mode until June 18, 1984, in the manual mode until January 16, 1995, and in the intrusion mode until May 3, 1995. A manual ENRAFTM system began recording on May 5, 1995. On July 8,1996 , the waste surface level was $4.53 \mathrm{~m}$ (178.5 in.), as measured by the manual ENRAF $^{\mathrm{TM}}$ system. The surface level readings from the manual ENRAF ${ }^{\mathrm{TM}}$ system increased approximately $300 \mathrm{~mm}$ (12 in.) between the January 2, 1996 and April 2, 1996 readings, because of a change in the baseline for the ENRAF ${ }^{\mathrm{TM}}$ system from the top of the dish to the tank's bottom center. Tank $241-\mathrm{U}-108$ is not an assumed leaker. A graphical representation of the volume measurements is presented as a level history graph in Figure A4-1.

\section{A4.2 DRYWELL READINGS}

Tank 241-U-108 has four dry wells. Only dry well $60-08-04$ (current readings $<200 \mathrm{c} / \mathrm{s}$ ) had readings greater than the $50 \mathrm{c} / \mathrm{s}$ background radiation.

\section{A4.3 INTERNAL TANK TEMPERATURES}

Tank 241-U-108 has a single thermocouple tree, located in riser 1 , with 11 thermocouples to monitor the waste temperature. Elevations are available for all of the thermocouples. Temperature data recorded from July 5, 1987 through October 13, 1996 were obtained from the Surveillance Analysis Computer System (SACS) (LMHC 1996) for all 11 thermocouples. The average temperature of the SACS data was $28.1^{\circ} \mathrm{C}\left(82.6^{\circ} \mathrm{F}\right)$, the minimum was $16.6^{\circ} \mathrm{C}\left(61.8^{\circ} \mathrm{F}\right)$, and the maximum was $37^{\circ} \mathrm{C}\left(98^{\circ} \mathrm{F}\right)$. The average temperature of the SACS data over the last year (October 1995 through October 1996$)$ was $28.3^{\circ} \mathrm{C}\left(82.6^{\circ} \mathrm{F}\right)$, 
Figure A4-1. Level History for Tank 241-U-108.

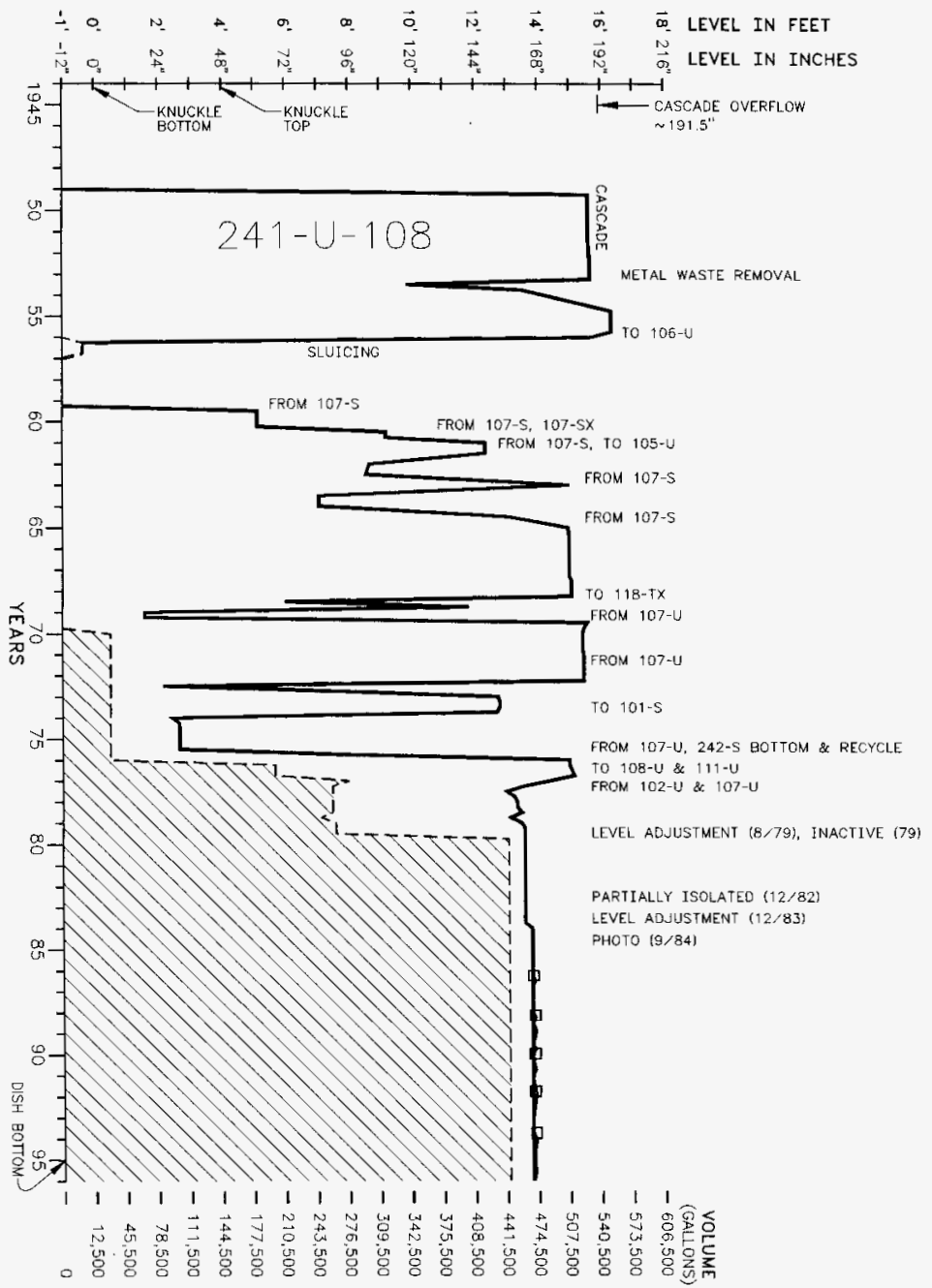


the minimum was $19.9^{\circ} \mathrm{C}\left(67.8^{\circ} \mathrm{F}\right)$, and the maximum was $33^{\circ} \mathrm{C}\left(91^{\circ} \mathrm{F}\right)$. A graph of the weekly high temperatures can be found in Figure A4-2. Plots of the individual thermocouple readings can be found in the Supporting Document for the Historical Tank Content Estimate for U Tank Farm (Brevick et al. 1994).

\section{A4.4 TANK 241-U-108 PHOTOGRAPHS}

The September 1984 photographic montage of tank 241-U-108's interior shows a dark surface of supernatant mixed with a tan-colored saltcake crust. In the foreground, a thermocouple tree has an accumulation of solids at the liquid surface. A recirculating dip tube and an overflow nozzle can be seen at the left. In the background, an FIC probe, a LOW, and a slurry pump can be observed. The bright white area near the temperature probe is the reflection from the camera light. The waste level has not changed since the photographs were taken; therefore, this photographic montage should accurately represent the tank waste's current appearance. 
Figure A4-2. Weekly High Temperature Plot for Tank 241-U-108.

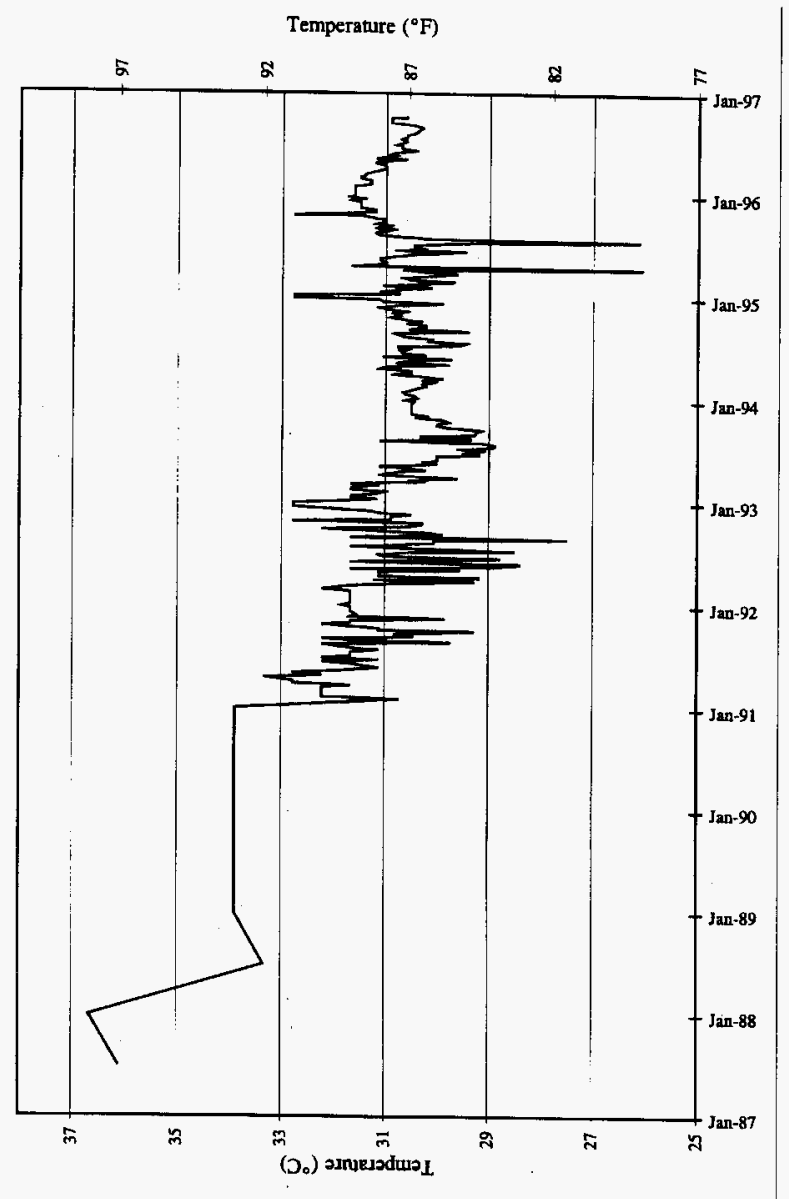




\section{A5.0 APPENDIX A REFERENCES}

Agnew, S.F., P. Baca, R. A. Corbin, T. B. Duran, and K. A. Jurgensen, 1996a, Waste Status and Transaction Record Summary for the Southwest Quadrant, WHC-SD-WM-TI-614, Rev. 2, Los Alamos National Laboratory, Los Alamos, New Mexico.

Agnew, S.F., J. Boyer, R. A. Corbin, T. B. Duran, J. R. Fitzpatrick, K. A. Jurgensen, T. P. Ortiz, and B. L. Young, 1996b, Hanford Tank Chemical and Radionuclide Inventories: HDW Model Rev. 3, LA-UR-96-858, Rev. 0, Los Alamos National Laboratory, Los Alamos, New Mexico.

Alstad, A. T., 1993, Riser Configuration Document for Single-Shell Waste Tanks, WHC-SD-RE-TI-053, Rev. 9, Westinghouse Hanford Company, Richland, Washington.

Brevick, C. H., L. A. Gaddis, and E. D. Johnson, 1994, Supporting Document for the Historical Tank Content Estimate for U Tank Farm, WHC-SD-WM-ER-325, Rev. 0, ICF Kaiser Hanford Company, Richland, Washington.

Hanlon, B. M., 1996, Waste Tank Summary Report for Month Ending September 30, 1996, WHC-EP-0182-102, Westinghouse Hanford Company, Richland, Washington.

LMHC, 1996, Surveillance Analysis Computer System In: SYBASE/Visual Basic [Mainframe]. Available: HLAN, Lockheed Martin Hanford Company, Richland, Washington.

Leach, C. E., and S. M. Stahl, 1996, Hanford Site Tank Farm Facilities Interim Safety Basis, WHC-SD-WM-ISB-001, Rev. OL, Westinghouse Hanford Company, Richland, Washington.

Lipnicki, J., 1996, Waste Tank Risers Available for Sampling, WHC-SD-WM-TI-710, Rev. 3, Westinghouse Hanford Company, Richland, Washington.

Rogers, R. D., and H. T. Daniels, 1944, Specifications for Construction of Composite Storage Tanks Bldg. \#241 at Hanford Engineer Works, CVI-73550, E. I. Du Pont de Nemours and Company, Richland, Washington.

Tran, T. T., 1993, Thermocouple Status Single Shell \& Double Shell Waste Tanks, WHC-SD-WM-TI-553, Rev. 0, Westinghouse Hanford Company, Richland, Washington. 
HNF-SD-WM-ER-639 Rev. 0

\section{APPENDIX B}

SAMPLING OF TANK 241-U-108 
HNF-SD-WM-ER-639 Rev. 0

This page left blank intentionally. 


\section{APPENDIX B}

\section{SAMPLING OF TANK 241-U-108}

This appendix provides sampling and analysis information for each known sampling event for tank 241-U-108 and provides an assessment of the core, grab, and vapor sampling results.

- Section B1: Tank Sampling Overview

- Section B2: Analytical Results

- Section B3: Assessment of Characterization Results

- Section B4: References for Appendix B.

Future sampling of tank 241-U-108 will be appended to the above list.

\section{B1.0 TANK SAMPLING OVERVIEW}

This section focuses on the April/May 1996 core sampling and analysis events for tank 241-U-108. Three core samples were taken to satisfy the requirements of the Tank Safety Screening Data Quality Objective (Dukelow et al. 1995), Data Quality Objective to Support Resolution of the Organic Complexant Safety Issue (Turner et al. 1996), Historical Model Evaluation Data Requirements (Simpson and McCain 1995), and Test Plan for Samples From Hanford Waste Tanks 241-BY-103, BY-104, BY-105, BY-106, BY-108, BY-110, TY-103, $U-105, U-107, U-108$, and $U-109$ (Meacham 1995). All three core samples were acquired and analyzed in accordance with the Tank 241-U-108 Push-Mode Core Sampling and Analysis Plan (SAP) (Homi 1996). Three liquid grab samples were taken in May/June 1995 to satisfy the requirements of Data Quality Objectives for the Waste Compatibility Program (Carothers 1994). Vapor samples were obtained in 1995 as required in Data Quality Objectives for Generic In-Tank Health and Safety Issue Resolution (Osborne et al. 1995). Additional sampling and analysis procedures can be found in the Tank Characterization Reference Guide (DeLorenzo et al. 1994).

Analytical results have also been reported for three "historical" sampling events: one in 1971, a liquid sample taken in late 1973 or early 1974, and a sludge sample taken in 1975. Because tank 241-U-108 was actively receiving waste until the first quarter of 1977 , sampling events prior to this date no longer represent the current tank contents. Data from sampling and analysis events prior to 1989 may not be acceptable for some regulatory evaluations and decisions. None of the three pre-1995 analytical data were used to evaluate 
the present tank contents because of the age of the data and the lack of specifics concerning sample locations. The data are included in this report for historical comparison. Results from historical sampling events are compared with recent analytical results to corroborate data and to identify data trends.

\section{B1.1 DESCRIPTION OF 1996 CORE AND 1995 GRAB SAMPLING EVENTS}

Core samples 141,145 , and 146, containing 9 segments each, were respectively collected from risers 7, 9, and 2 of tank 241-U-108 between April 5, 1996 and May 6, 1996. A field blank of deionized water was created and delivered to the 222-S Laboratory with the core 146 samples. All segments were taken in the push mode and received, extruded, and analyzed at the 222-S Laboratories to support the requirements of the safety screening, organic, and historical DQO documents, and the organic test plan. Before taking each core sample, the flammability of tank vapors was checked using a flammable gas meter. This check was made to meet operational safety requirements and to fulfill the safety screening DQO requirement. To meet these requirements, the vapors in the tank headspace must be less than 25 percent of the lower flammability limit (LFL). This measurement was conducted in the field and was recorded in the work package (WHC 1996). The highest combustible gas meter reading observed from multiple readings in each of the three risers used for sampling was 8.6 percent of the LFL, observed in the headspace below riser 9 at 1115 hours on April 26, 1996.

Three liquid grab samples, U-108-1, U-108-2, and U-108-3, were removed from riser 7 of tank 241-U-108 on May 31, 1995 and received by the 222-S Laboratory on June 1, 1995. Sample U-108-1 was analyzed to support the waste compatibility safety issue. The remaining two samples were archived for possible future analysis.

The sampling and analytical requirements of the DQO documents covering the core, grab, and vapor samples are summarized in Table B1-1.

\section{B1.2 SAMPLE HANDLING (1996 CORE AND 1995 GRAB SAMPLES)}

Pertinent sampling information for the three core samples is provided in Tables B1-2, B1-3, and B1-4. Upon delivery to the 222-S Laboratory, cores 141,145 , and 146 were extruded and subsampled as prescribed in the SAP (Homi 1996). Video recordings and/or color photographs were taken of each segment immediately following extrusion and may be viewed by contacting the 222-S Laboratory.

The sampling and analysis schemes for the solids and liquids recovered from the three cores are provided in Homi (1996). When more than a $24-\mathrm{cm}$ (9-in.) half segment was extruded, solid material from the extruded segments were divided into half segments labeled as upper and lower halves. The upper half is the material in the top half of the sampler; the material 
collected first by the sampler. Drainable liquids were identified as such. Core composites were created for all three cores in accordance with the historical DQO.

Table B1-1. Integrated Data Quality Objective Requirements for Tank 241-U-108. ${ }^{1}$

\begin{tabular}{|c|c|c|c|}
\hline . & (10) & 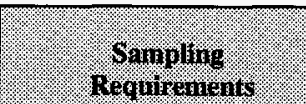 & 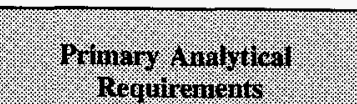 \\
\hline $\begin{array}{l}\text { Core samples } \\
141,145,146\end{array}$ & $\begin{array}{l}\text { Safety } \\
\text { screening }\end{array}$ & $\begin{array}{l}\text { Core samples from a } \\
\text { minimum of three }{ }^{2} \text { risers } \\
\text { separated radially to the } \\
\text { maximum extent possible }\end{array}$ & $\begin{array}{l}\text { Energetics, moisture content, } \\
\text { total alpha, bulk density, } \\
\text { flammable gas }\end{array}$ \\
\hline $\begin{array}{l}\text { Core samples } \\
141,145,146\end{array}$ & Organic & $\begin{array}{l}\text { Core samples from three }{ }^{2} \\
\text { risers }\end{array}$ & $\begin{array}{l}\text { Energetics, moisture content, } \\
\text { nickel, total organic carbon }\end{array}$ \\
\hline $\begin{array}{l}\text { Core samples } \\
141,145,146\end{array}$ & Historical & $\begin{array}{l}\text { Core samples from three }{ }^{2} \\
\text { risers }\end{array}$ & $\begin{array}{l}\text { Energetics, moisture, total } \\
\text { beta, anions, metals, uranium, } \\
\text { Cs-137, Sr-90, bulk density }\end{array}$ \\
\hline $\begin{array}{l}\text { Core samples } \\
141,145,146\end{array}$ & $\begin{array}{l}\text { Organic test } \\
\text { plan }\end{array}$ & $\begin{array}{l}\text { Core samples from three } \\
\text { risers }\end{array}$ & Energetics, moisture, TOC \\
\hline $\begin{array}{l}1995 \text { Grab } \\
\text { samples }\end{array}$ & $\begin{array}{l}\text { Waste } \\
\text { compatibility }\end{array}$ & $\begin{array}{l}\text { Liquid samples from one } \\
\text { riser at three depths }\end{array}$ & $\begin{array}{l}\text { Energetics, moisture content, } \\
\text { total organic carbon, Cs-137, } \\
\mathrm{Sr}-90, \mathrm{Al}, \mathrm{Fe}, \mathrm{Na}, \text { hydroxide, } \\
\text { anions, carbonate, pH, } \\
\mathrm{Pu}-239 / 240, \mathrm{Am}-241 \text {, specific } \\
\text { gravity, volume percent solids }\end{array}$ \\
\hline \multirow[t]{3}{*}{$\begin{array}{l}\text { Vapor } \\
\text { samples }\end{array}$} & $\begin{array}{l}\text { Generic } \\
\text { in-tank health } \\
\text { and safety }\end{array}$ & \multirow[t]{3}{*}{ See Table B1-6 } & \multirow[t]{3}{*}{$\begin{array}{l}\text { Organic vapors, hydrogen, } \\
\text { nitrous oxide, carbon dioxide, } \\
\text { carbon monoxide, ammonia, } \\
\text { nitrogen dioxide, nitric oxide, } \\
\text { water vapor }\end{array}$} \\
\hline & $\begin{array}{l}\text { Rotary core } \\
\text { vapor }\end{array}$ & & \\
\hline & $\begin{array}{l}\text { Organic } \\
\text { solvent } \\
\text { screening }\end{array}$ & & \\
\hline
\end{tabular}

Notes:

'Winkelman (1996)

${ }^{2}$ No applicable DQOs required more than two core samples; however, Tank Waste Characterization Basis (Brown et al. 1995) required that three cores be taken.

One grab sample was broken down and subsampled for compatibility according to Schreiber (1995) following delivery to the 222-S Laboratory. Visual inspection of sample U-108-1 indicated that the sample contained less than 2 percent settled solids and that no organic layer was present. The remaining grab samples were archived. 
Table B1-2. Sample Receipt and Extrusion Information for Tank 241-U-108, Core 141, Riser 7. (2 sheets)

\begin{tabular}{|c|c|c|c|c|c|c|c|}
\hline sosin & 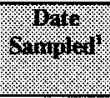 & 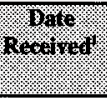 & Mare: & Whom & 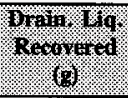 & 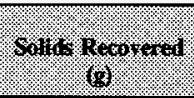 & 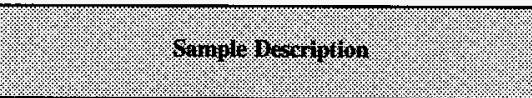 \\
\hline 1 & $4 / 5 / 96$ & $4 / 16 / 96$ & $4 / 23 / 96$ & 0.0 & 152.5 & 84.1 (lower half) & $\begin{array}{l}\text { Approximately } 100 \mathrm{~mL} \text { of drainable liquid were } \\
\text { collected. The liquid was yellow but clear, and was } \\
\text { placed into one jar. Approximately } 10 \mathrm{~cm} \text { ( } 4 \text { in.) of } \\
\text { shudge, dark gray in color, were extruded. The } \\
\text { sludge was subsampled in one jar. }\end{array}$ \\
\hline 2 & $4 / 8 / 96$ & $4 / 16 / 96$ & $4 / 22 / 96$ & 0.0 & 0.0 & 195.0 (upper half) & $\begin{array}{l}\text { About } 19 \mathrm{~cm}(7.5 \mathrm{in} \text {.) of solids were extruded. The } \\
\text { solids were collected in one jar and were gray in } \\
\text { color. The texture resembled a mixture of sludge and } \\
\text { saltcake. }\end{array}$ \\
\hline 3 & $4 / 8 / 96$ & $4 / 16 / 96$ & $4 / 22 / 96$ & 0.0 & 0.0 & 129.5 (upper half) & $\begin{array}{l}\text { About } 10 \mathrm{~cm}(4 \mathrm{in} .) \text { of solids were extruded. The } \\
\text { solids were medium gray in color and the texture } \\
\text { resembled a mixture of sludge and saltcake. }\end{array}$ \\
\hline $3 \mathrm{~A}$ & $4 / 9 / 96$ & $4 / 16 / 96$ & $4 / 22 / 96$ & 0.0 & 0.0 & 85.7 (upper half) & $\begin{array}{l}\text { Approximately } 10 \mathrm{~cm}(4 \mathrm{in} \text {.) of solids were collected } \\
\text { in one jar. The solids were medium gray in color and } \\
\text { the texture resembled a mixture of sludge and } \\
\text { saltcake. }\end{array}$ \\
\hline 4 & $4 / 15 / 96$ & $4 / 16 / 96$ & $4 / 23 / 96$ & 0.0 & 0.0 & $\begin{array}{l}145.3 \text { (upper half) } \\
214.2 \text { (lower half) }\end{array}$ & $\begin{array}{l}\text { Roughly } 38 \mathrm{~cm} \text { ( } 15 \mathrm{in} \text {.) of solids were recovered. } \\
\text { The solids were blue gray in color and the texture } \\
\text { resembled a mixture of sludge and saltcake. The } \\
\text { solids were divided into upper and lower half } \\
\text { subsegments. }\end{array}$ \\
\hline $4 \mathrm{~A}$ & $4 / 15 / 96$ & $4 / 16 / 96$ & $4 / 24 / 96$ & 0.0 & 0.0 & $\begin{array}{c}59.4 \text { (upper half) } \\
212.7 \text { (lower half) }\end{array}$ & $\begin{array}{l}28 \mathrm{~cm} \text { (11 in.) of solids, dark gray in color and } \\
\text { resembling a sludge, were extruded. The solids were } \\
\text { divided into upper and lower half subsegments. }\end{array}$ \\
\hline 5 & $4 / 15 / 96$ & $4 / 23 / 96$ & $4 / 29 / 96$ & 0.0 & 0.0 & $\begin{array}{l}242.7 \text { (upper half) } \\
237.7 \text { (lower half) }\end{array}$ & $\begin{array}{l}48 \mathrm{~cm} \text { (19 in.) of solids were collected. The solids } \\
\text { were a damp, crystalline, bluish-gray saltcake, and } \\
\text { were partitioned in upper and lower halves. }\end{array}$ \\
\hline
\end{tabular}


Table B1-2. Sample Receipt and Extrusion Information for Tank 241-U-108, Core 141, Riser 7. (2 sheets)

\begin{tabular}{|c|c|c|c|c|c|c|c|}
\hline 88 & Orrover, & Merainged & Binition & Wopto & 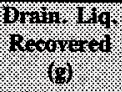 & 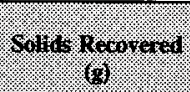 & 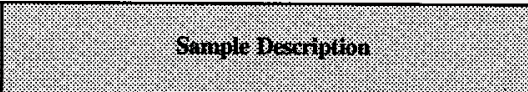 \\
\hline 6 & $4 / 18 / 96$ & $4 / 23 / 96$ & $4 / 29 / 96$ & 0.0 & 0.0 & $\begin{array}{l}226.6 \text { (upper half) } \\
260.0 \text { (lower half) }\end{array}$ & $\begin{array}{l}48 \mathrm{~cm} \text { ( } 19 \text { in.) of solids were collected. The solids } \\
\text { were medium bluish gray in color and were a damp, } \\
\text { crystalline saltcake. They were divided into upper } \\
\text { and lower half subsegments. }\end{array}$ \\
\hline 7 & $4 / 19 / 96$ & $4 / 30 / 96$ & $5 / 3 / 96$ & 0.0 & 0.0 & $\begin{array}{l}245.8 \text { (upper half) } \\
208.3 \text { (lower half) }\end{array}$ & $\begin{array}{l}\text { A full segment was collected as upper and lower half } \\
\text { subsegments. The wet solids were medium gray in } \\
\text { color and appeared as a sludge and saltcake mix. }\end{array}$ \\
\hline 8 & $4 / 19 / 96$ & $4 / 30 / 96$ & $5 / 3 / 96$ & 0.0 & 0.0 & $\begin{array}{l}186.5 \text { (upper half) } \\
230.8 \text { (lower half) }\end{array}$ & 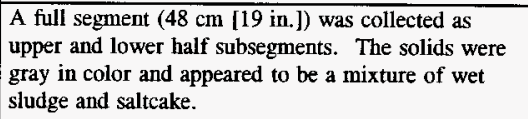 \\
\hline 9 & $4 / 19 / 96$ & $4 / 23 / 96$ & $5 / 6 / 96$ & 0.0 & 0.0 & 193.5 (upper half) & $\begin{array}{l}\text { Approximately } 15 \mathrm{~cm} \text { (6 in.) of solids were collected } \\
\text { into one jar. The solids were gray/white in color and } \\
\text { appeared to be a moist saltcake. Less than } 5 \mathrm{~mL} \text { of } \\
\text { liner liquid was observed but it was not retained. }\end{array}$ \\
\hline
\end{tabular}

Note:

${ }^{1}$ Dates are in $\mathrm{mm} / \mathrm{dd} / \mathrm{yy}$ format. 
Table B1-3. Sample Receipt and Extrusion Information for Tank 241-U-108, Core 145, Riser 9. (2 sheets)

\begin{tabular}{|c|c|c|c|c|c|c|c|}
\hline 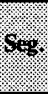 & 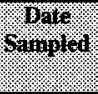 & ondo & 3\%+10\% & $\log$ & 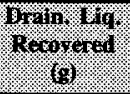 & 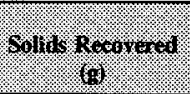 & 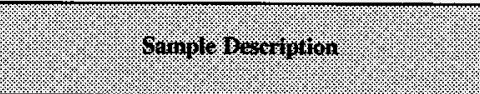 \\
\hline 1 & $4 / 23 / 96$ & $4 / 30 / 96$ & $5 / 6 / 96$ & 0.0 & 168.1 & 0.0 & $\begin{array}{l}\text { Approximately } 120 \mathrm{~mL} \text { of drainable liquid were } \\
\text { collected. The liquid was dark gray in color and } \\
\text { opaque. No solids were present. }\end{array}$ \\
\hline 2 & $4 / 23 / 96$ & $4 / 30 / 96$ & $5 / 7 / 96$ & 0.0 & 0.0 & 194.2 (upper half) & $\begin{array}{l}\text { Approximately } 18 \mathrm{~cm} \text { ( } 7 \text { in.) of solids were } \\
\text { extruded. The solids were medium gray in color } \\
\text { and the texture resembled a salt slurry. The } \\
\text { solids were subsampled into one jar. }\end{array}$ \\
\hline 3 & $4 / 25 / 96$ & $5 / 10 / 96$ & $5 / 14 / 96$ & 0.0 & 0.0 & $\begin{array}{l}207.7 \text { (upper half) } \\
182.7 \text { (lower half) }\end{array}$ & $\begin{array}{l}\text { Approximately } 14 \text { in. of wet saltcake, light to } \\
\text { dark gray in color, were extruded. The solids } \\
\text { were partitioned into upper and lower halves. }\end{array}$ \\
\hline 4 & $4 / 25 / 96$ & $5 / 10 / 96$ & $5 / 14 / 96$ & 0.0 & 0.0 & $\begin{array}{l}212.9 \text { (upper half) } \\
184.8 \text { (lower half) }\end{array}$ & $\begin{array}{l}\text { Approximately } 38 \mathrm{~cm}(15 \mathrm{in} .) \text { of medium gray, } \\
\text { wet saltcake were collected as upper and lower } \\
\text { half portions. }\end{array}$ \\
\hline 5 & $4 / 25 / 96$ & $5 / 10 / 96$ & $5 / 14 / 96$ & 0.0 & 0.0 & $\begin{array}{l}253.7 \text { (upper half) } \\
226.6 \text { (lower half) }\end{array}$ & $\begin{array}{l}\text { A full segment ( } 48 \mathrm{~cm} \text { [19 in.]) was collected as } \\
\text { upper and lower half subsegments. The wet } \\
\text { saltcake was dark gray in color and was } \\
\text { subsampled as upper and lower halves. }\end{array}$ \\
\hline 6 & $4 / 25 / 96$ & $4 / 30 / 96$ & $5 / 7 / 96$ & 0.0 & 0.0 & $\begin{array}{l}249.0 \text { (upper half) } \\
248.3 \text { (lower half) }\end{array}$ & $\begin{array}{l}\text { A full segment ( } 48 \mathrm{~cm} \text { [19 in.]) was collected as } \\
\text { upper and lower half subsegments. The moist } \\
\text { saltcake was medium gray in color and was } \\
\text { subsampled as upper and lower halves. }\end{array}$ \\
\hline
\end{tabular}


Table B1-3. Sample Receipt and Extrusion Information for Tank 241-U-108, Core 145, Riser 9. (2 sheets)

\begin{tabular}{|c|c|c|c|c|c|c|c|}
\hline $4 \%$ & Painter & $\operatorname{lochlog}$ & Berogides & 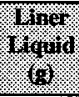 & 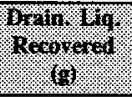 & folitis heoriver & Shinilo Drocinition \\
\hline 7 & $4 / 25 / 96$ & $4 / 30 / 96$ & $5 / 14 / 96$ & 0.0 & 0.0 & $\begin{array}{l}261.8 \text { (upper half) } \\
217.9 \text { (lower half) }\end{array}$ & $\begin{array}{l}\text { Approximately } 48 \mathrm{~cm} \text { ( } 19 \mathrm{in} \text {.) of wet saltcake, } \\
\text { medium gray in color, were extruded. The core } \\
\text { was divided into upper and lower halves. }\end{array}$ \\
\hline 8 & $4 / 26 / 96$ & $4 / 30 / 96$ & $5 / 8 / 96$ & 0.0 & 0.0 & $\begin{array}{l}249.9 \text { (upper half) } \\
225.3 \text { (lower half) }\end{array}$ & $\begin{array}{l}\text { Approximately } 48 \mathrm{~cm} \text { ( } 19 \text { in.) of moist saltcake, } \\
\text { medium gray in color, were extruded. The core } \\
\text { was divided into upper and lower halves. Less } \\
\text { than } 5 \mathrm{~mL} \text { of liner liquid were observed but not } \\
\text { retained. }\end{array}$ \\
\hline 9 & $4 / 26 / 96$ & $4 / 30 / 96$ & $5 / 15 / 96$ & 0.0 & 0.0 & $\begin{array}{l}167.7 \text { (upper half) } \\
32.0 \text { (lower half) }\end{array}$ & $\begin{array}{l}\text { Roughly } 23 \mathrm{~cm} \text { ( } 9 \text { in.) of solids were collected. } \\
\text { The upper portion was } 20 \mathrm{~cm}(8 \mathrm{in} \text {.) of wet } \\
\text { sludge and was dark gray in color. The lower } \\
2.5 \text {-cm (1-in.) portion was moist saltcake that } \\
\text { was medium gray in color. The two portions } \\
\text { were placed in separate jars. }\end{array}$ \\
\hline
\end{tabular}


Table B1-4. Sample Receipt and Extrusion Information for Tank 241-U-108, Core 146, Riser 2. (2 sheets)

\begin{tabular}{|c|c|c|c|c|c|c|c|}
\hline Sese. & Sonoled & Hecinted & mavidad & Giond & 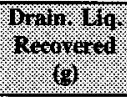 & 6rofids recoriared & 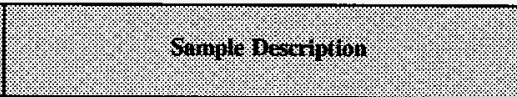 \\
\hline FB & $4 / 30 / 96$ & $5 / 3 / 96$ & $5 / 7 / 96$ & 0.0 & 233.8 & 0.0 & $\begin{array}{l}\text { Roughly } 250 \mathrm{~mL} \text { of clear, colorless drainable liquid } \\
\text { were collected. }\end{array}$ \\
\hline 1 & $5 / 1 / 96$ & $5 / 10 / 96$ & $5 / 14 / 96$ & 0.0 & 85.0 & 177.9 (upper half) & $\begin{array}{l}70 \mathrm{~mL} \text { of dark gray, opaque drainable liquid were } \\
\text { collected. In addition, approximately } 18 \mathrm{~cm} \text { ( } 7 \mathrm{in} \text {.) } \\
\text { of dark gray, wet saltcake were collected. }\end{array}$ \\
\hline 2 & $5 / 1 / 96$ & $5 / 17 / 96$ & $5 / 21 / 96$ & 0.0 & 0.0 & $\begin{array}{l}139.9 \text { (upper half) } \\
176.1 \text { (lower half) }\end{array}$ & $\begin{array}{l}\text { About } 33 \mathrm{~cm}(13 \mathrm{in} .) \text { of light to dark gray, dry } \\
\text { saltcake were collected. The sample was partitioned } \\
\text { in upper and lower half portions. }\end{array}$ \\
\hline 3 & $5 / 1 / 96$ & $5 / 16 / 96$ & $5 / 22 / 96$ & 0.0 & 0.0 & $\begin{array}{l}80.2 \text { (upper half) } \\
104.4 \text { (lower half) }\end{array}$ & $\begin{array}{l}\text { About } 16 \mathrm{~cm}(6.5 \mathrm{in} \text {.) of gray, moist saltcake were } \\
\text { extruded. The sample was divided into upper and } \\
\text { lower halves. }\end{array}$ \\
\hline $3 \mathrm{~A}$ & $5 / 3 / 96$ & $5 / 3 / 96$ & $5 / 7 / 96$ & 0.0 & 0.0 & 249.4 (whole) & $\begin{array}{l}\text { Approximately } 23 \mathrm{~cm}(9 \mathrm{in} \text {.) of gray, moist saltcake } \\
\text { were collected. All solids were placed into one jar. }\end{array}$ \\
\hline 4 & $5 / 3 / 96$ & $5 / 3 / 96$ & $5 / 7 / 96$ & 0.0 & 0.0 & $\begin{array}{l}141.8 \text { (upper half) } \\
162.9 \text { (lower half) }\end{array}$ & $\begin{array}{l}\text { Approximately } 35 \mathrm{~cm}(14 \mathrm{in} .) \text { of medium gray, } \\
\text { moist saltcake were collected as upper and lower } \\
\text { halves. }\end{array}$ \\
\hline 5 & $5 / 3 / 96$ & $5 / 3 / 96$ & $5 / 7 / 96$ & 0.0 & 0.0 & $\begin{array}{l}241.0 \text { (upper half) } \\
216.8 \text { (lower half) }\end{array}$ & $\begin{array}{l}\text { Extruded approximately } 46 \mathrm{~cm} \text { ( } 18 \text { in.) of medium } \\
\text { gray, wet saltcake were extruded, and partitioned } \\
\text { into upper and lower half subsegments. }\end{array}$ \\
\hline 6 & $5 / 3 / 96$ & $5 / 14 / 96$ & $5 / 15 / 96$ & 0.0 & 0.0 & $\begin{array}{l}260.1 \text { (upper half) } \\
243.4 \text { (lower half) }\end{array}$ & $\begin{array}{l}\text { A full segment of medium gray, wet saltcake was } \\
\text { extruded and divided into upper and lower half } \\
\text { subsegments. }\end{array}$ \\
\hline
\end{tabular}


Table B1-4. Sample Receipt and Extrusion Information for Tank 241-U-108, Core 146, Riser 2. (2 sheets)

\begin{tabular}{|c|c|c|c|c|c|c|c|}
\hline 6. & 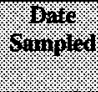 & 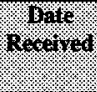 & Gringat & Hines & 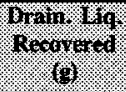 & 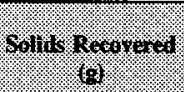 & 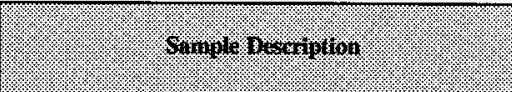 \\
\hline 7 & $5 / 3 / 96$ & $5 / 14 / 96$ & $5 / 15 / 96$ & 0.0 & 0.0 & $\begin{array}{l}232.9 \text { (upper half) } \\
237.8 \text { (lower half) }\end{array}$ & $\begin{array}{l}\text { About } 19 \text { in, of gray, moist saltcake, was collected } \\
\text { and divided in upper and lower half subsegments. }\end{array}$ \\
\hline 8 & $5 / 3 / 96$ & $5 / 17 / 96$ & $5 / 22 / 96$ & 0.0 & 0.0 & $\begin{array}{l}229.4 \text { (upper half) } \\
220.4 \text { (lower half) }\end{array}$ & $\begin{array}{l}\text { Approximately } 46 \mathrm{~cm} \text { ( } 18 \mathrm{in} \text {.) of gray, wet saltcake } \\
\text { was extruded. The sample was divided in upper and } \\
\text { lower halves. }\end{array}$ \\
\hline 9 & $5 / 6 / 96$ & $5 / 14 / 96$ & $5 / 15 / 96$ & 0.0 & 0.0 & $\begin{array}{l}105.1 \text { (qtr seg. A) } \\
112.7 \text { (lower half) }\end{array}$ & $\begin{array}{l}\text { Roughly } 23 \mathrm{~cm}(9 \mathrm{in} \text {.) of solids were extruded. The } \\
\text { upper } 10 \mathrm{~cm} \text { ( } 4 \text { in.) were gray, wet, sludge collected } \\
\text { as the upper quarter segment. The remaining } 13 \mathrm{~cm} \\
\text { ( } 5 \text { in.) were light to medium gray, moist saltcake. } \\
\text { They were collected as the lower half subsegment. }\end{array}$ \\
\hline
\end{tabular}

Note:

$\mathrm{FB}=$ field blank 


\section{B1.3 SAMPLE ANALYSIS (1996 CORE AND 1995 GRAB SAMPLES)}

The analyses performed at the half segment level on solid samples, at the segment level on drainable liquid samples, and on the core composite samples from cores 141, 145, and 146 were not limited to those needed to satisfy the safety screening, organic, and historical DQO requirements. Additional analytical results for metals, anions, and radionuclides were obtained on an opportunistic basis (Kristofzski 1996) in the process of meeting the DQO requirements. The waste compatibility DQO requirements were performed on a 1995 liquid grab sample. The results of those analyses were limited to those necessary to satisfy the DQO document. Core and grab sample analyses results have been reported in Bell (1996a) and Esch (1995) respectively.

Depending on the analysis, solid subsamples were analyzed directly or after a fusion, acid, or water digestion. Drainable liquid core subsamples and liquid grab subsamples were analyzed directly or after dilution in water or acid. Analysis for percent of the LFL, bulk density, total organic carbon (TOC), and total inorganic carbon (TIC) was performed directly. Analysis by thermogravimetric analysis (TGA) and differential scanning calorimetry (DSC) was performed directly under a nitrogen purge. One sample from core 141 exceeded the DSC notification limit specified in the core SAP and an immediate notification was made (Bell 1996b). No other notification limits were exceeded.

Total alpha activity, inductively coupled plasma (ICP), strontium-90 (Sr-90), cesium-137 (Cs-137), total beta, and total uranium measurements were performed on samples that had been fused with potassium hydroxide and then dissolved in acid. Ion chromatography (IC) and inductively coupled plasma spectroscopy (ICP) on the core composites were performed on samples that had been prepared by water digestion. Analysis by ICP was also performed on acid-digested core composite samples. Analysis of fusion-digested samples requires a high dilution, resulting in higher detection limits. Analysis of acid-digested samples allows quantification of some trace elements that are not quantified in the fusion digests.

The results of the analyses are presented and discussed in Section B2.0. The results of the quality control (QC) tests and the implications for data quality are discussed in Section B3.2. Table B1-5 is a summary of the cores, segments, segment portions, individual sample numbers, and the analyses performed on each sample. All reported analyses were performed in accordance with approved laboratory procedures. The procedure numbers are presented in the discussion in Section B2.0. 
Table B1-5. Tank 241-U-103 Sample Analysis Summary. ( 8 sheets)

\begin{tabular}{|c|c|c|c|}
\hline (6.10) & 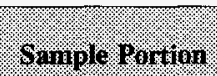 & WHinow & whalyos \\
\hline \multirow[t]{25}{*}{141} & $\begin{array}{l}\text { Segment } 1 \text {, } \\
\text { drainable liq. }\end{array}$ & S96T002282 & $\begin{array}{l}\text { DSC, TGA, specific gravity, TOC, TIC, ICP, } \\
\text { IC, total alpha }\end{array}$ \\
\hline & \multirow{4}{*}{$\begin{array}{l}\text { Segment } 1 \text {, lower } \\
\text { half }\end{array}$} & S96T002270 & IC \\
\hline & & S96T002230 & Bulk density \\
\hline & & S96T002241 & DSC, TGA, TOC, TIC \\
\hline & & S96T002257 & ICP-fusion, GEA, total alpha \\
\hline & \multirow{4}{*}{$\begin{array}{l}\text { Segment 2, upper } \\
\text { half }\end{array}$} & S96T002231 & Bulk density \\
\hline & & S96T002273 & IC \\
\hline & & S96T002242 & DSC, TGA, TOC, TIC \\
\hline & & S96T002258 & ICP-fusion, GEA, total alpha \\
\hline & \multirow{4}{*}{$\begin{array}{l}\text { Segment 3, upper } \\
\text { half }\end{array}$} & S96T002232 & Bulk density \\
\hline & & S96T002274 & IC \\
\hline & & S96T002243 & DSC, TGA, TOC, TIC \\
\hline & & S96T002259 & ICP-fusion, GEA, total alpha \\
\hline & \multirow{4}{*}{$\begin{array}{l}\text { Segment } 3 A \text {, } \\
\text { upper half }\end{array}$} & S96T002233 & Bulk density \\
\hline & & S96T002275 & IC \\
\hline & & S96T002244 & DSC, TGA, TOC, TIC \\
\hline & & S96T002260 & ICP-fusion, GEA, total alpha \\
\hline & \multirow{4}{*}{$\begin{array}{l}\text { Segment } 4 \text {, lower } \\
\text { half }\end{array}$} & S96T002235 & Bulk density \\
\hline & & S96T002277 & $\mathrm{IC}$ \\
\hline & & S96T002246 & DSC, TGA, TOC, TIC \\
\hline & & S96T002262 & ICP-fusion, GEA, total alpha \\
\hline & \multirow{4}{*}{$\begin{array}{l}\text { Segment } 4 \text {, upper } \\
\text { half }\end{array}$} & S96T002234 & Bulk density \\
\hline & & S96T002276 & IC \\
\hline & & S96T002245 & DSC, TGA, TOC, TIC \\
\hline & & S96T002261 & ICP-fusion, GEA, total alpha \\
\hline
\end{tabular}


Table B1-5. Tank 241-U-103 Sample Analysis Summary. (8 sheets)

\begin{tabular}{|c|c|c|c|}
\hline Oore & Sample gortion. & Somplo & Anarysis: \\
\hline \multirow{28}{*}{$\begin{array}{l}141 \\
\text { (Cont'd) }\end{array}$} & \multirow{4}{*}{$\begin{array}{l}\text { Segment } 4 A \text {, } \\
\text { lower half }\end{array}$} & S96T002237 & Bulk density \\
\hline & & S967002279 & $\overline{\mathrm{IC}}$ \\
\hline & & S96T002248 & DSC, TGA, TOC, TIC \\
\hline & & S96T002264 & ICP-fusion, GEA, total alpha \\
\hline & \multirow{4}{*}{$\begin{array}{l}\text { Segment } 4 A \text {, } \\
\text { upper half }\end{array}$} & S96T002236 & Bulk density \\
\hline & & S96T002278 & $\overline{\mathrm{IC}}$ \\
\hline & & S96T002247 & $\overline{\mathrm{DSC}}, \overline{\mathrm{TG}} \mathrm{A}, \overline{\mathrm{TOC}}, \overline{\mathrm{TIC}}$ \\
\hline & & S96T002263 & ICP-fusion, GEA, total alpha \\
\hline & \multirow{4}{*}{$\begin{array}{l}\text { Segment } 5 \text {, lower } \\
\text { half }\end{array}$} & S96T002563 & Bulk density \\
\hline & & S96T002586 & DSC, TGA, TOC, TIC \\
\hline & & S96T002618 & IC \\
\hline & & S96T002609 & ICP-fusion, GEA, total alpha \\
\hline & \multirow{4}{*}{$\begin{array}{l}\text { Segment 5, upper } \\
\text { half }\end{array}$} & \$96T002562 & Bulk density \\
\hline & & S96T002617 & IC \\
\hline & & S96T002585 & DSC, TGA, TOC, TIC \\
\hline & & S96T002608 & ICP-fusion, GEA, total alpha \\
\hline & \multirow{4}{*}{$\begin{array}{l}\text { Segment } 6 \text {, lower } \\
\text { half }\end{array}$} & S96T002565 & Bulk density \\
\hline & & S96T002620 & IC \\
\hline & & S96T002588 & DSC, TGA, TOC, TIC \\
\hline & & S96T002611 & ICP-fusion, GEA, total alpha \\
\hline & \multirow{4}{*}{$\begin{array}{l}\text { Segment } 6 \text {, upper } \\
\text { half }\end{array}$} & S96T002564 & Bulk density \\
\hline & & S96T002619 & $\mathrm{IC}$ \\
\hline & & S96T002587 & DSC, TGA, TOC, TIC \\
\hline & & S96T002610 & ICP-fusion, GEA, total alpha \\
\hline & \multirow{4}{*}{$\begin{array}{l}\text { Segment 7, lower } \\
\text { half }\end{array}$} & S96T002567 & Bulk density \\
\hline & & S96T002622 & IC \\
\hline & & S96T002590 & $\overline{\mathrm{DSC}}, \mathrm{TGA}, \mathrm{TOC}, \overline{\mathrm{TIC}}$ \\
\hline & & S96T002613 & ICP-fusion, GEA, total alpha \\
\hline
\end{tabular}


Table B1-5. Tank 241-U-103 Sample Analysis Summary. (8 sheets)

\begin{tabular}{|c|c|c|c|}
\hline Core: & Sample fortinn: & Sumples & analisis: \\
\hline \multirow[t]{22}{*}{141} & \multirow{4}{*}{$\begin{array}{l}\text { Segment } 7 \text {, upper } \\
\text { half }\end{array}$} & S96T002566 & Bulk density \\
\hline & & S96T002621 & IC \\
\hline & & S96T002589 & DSC, TGA, TOC, TIC \\
\hline & & S96T002612 & ICP-fusion, GEA, total alpha \\
\hline & \multirow{4}{*}{$\begin{array}{l}\text { Segment } 8 \text {, lower } \\
\text { half }\end{array}$} & S96T002569 & Bulk density \\
\hline & & S96T002624 & $\overline{\mathrm{IC}}$ \\
\hline & & S96T002592 & DSC, TGA, TOC, TIC \\
\hline & & S96T002615 & ICP-fusion, GEA, total alpha \\
\hline & \multirow{4}{*}{$\begin{array}{l}\text { Segment } 8 \text {, upper } \\
\text { half }\end{array}$} & S96T002568 & Bulk density \\
\hline & & S96T002623 & IC \\
\hline & & S96T002591 & DSC, TGA, TOC, TIC \\
\hline & & S96T002614 & ICP-fusion, GEA, total alpha \\
\hline & \multirow{4}{*}{$\begin{array}{l}\text { Segment 9, upper } \\
\text { half }\end{array}$} & S96T002570 & Bulk density \\
\hline & & S96T002625 & IC \\
\hline & & S96T002593 & DSC, TGA, TOC, TIC \\
\hline & & S96T002616 & ICP-fusion, GEA, total alpha \\
\hline & \multirow{6}{*}{$\begin{array}{l}\text { Solid core } \\
\text { composite }\end{array}$} & S96T003448 & Bulk density \\
\hline & & S96T003449 & DSC, TGA, TOC, TIC \\
\hline & & S96T003452 & IC \\
\hline & & S96T003451 & ICP-Acid \\
\hline & & S96T003450 & $\begin{array}{l}\text { ICP-fusion, GEA, total uranium, total beta, } \\
\text { Sr-90 }\end{array}$ \\
\hline & & S96T003453 & ICP-Water \\
\hline \multirow[t]{9}{*}{145} & $\begin{array}{l}\text { Segment } 1, \\
\text { drainable liq. }\end{array}$ & S96T002942 & $\begin{array}{l}\text { DSC, TGA, specific gravity, TOC, TIC, ICP, } \\
\text { IC, total alpha }\end{array}$ \\
\hline & \multirow{4}{*}{$\begin{array}{l}\text { Segment } 2 \text {, upper } \\
\text { half }\end{array}$} & S96T002864 & Bulk density \\
\hline & & S96T002893 & DSC, TGA, TOC, TIC \\
\hline & & S96T002924 & IC \\
\hline & & S96T002909 & ICP-fusion, GEA, total alpha \\
\hline & \multirow{4}{*}{$\begin{array}{l}\text { Segment 3, lower } \\
\text { half }\end{array}$} & S96T002866 & Bulk density \\
\hline & & S96T002880 & DSC, TGA, TOC, TIC \\
\hline & & S96T002926 & IC \\
\hline & & S96T002911 & ICP-fusion, GEA, total alpha \\
\hline
\end{tabular}


Table B1-5. Tank 241-U-103 Sample Analysis Summary. (8 sheets)

\begin{tabular}{|c|c|c|c|}
\hline Coro: & Simple Torition & Sianiple & analisis \\
\hline \multirow{32}{*}{$\begin{array}{l}145 \\
\text { (Cont'd) }\end{array}$} & \multirow{4}{*}{$\begin{array}{l}\text { Segment 3, upper } \\
\text { half }\end{array}$} & S96T002865 & Bulk density \\
\hline & & S96T002879 & DSC, TGA, TOC, TIC \\
\hline & & S96T002925 & IC \\
\hline & & S96T002910 & ICP-fusion, GEA, total alpha \\
\hline & \multirow{4}{*}{$\begin{array}{l}\text { Segment } 4 \text {, lower } \\
\text { half }\end{array}$} & S96T002868 & Bulk density \\
\hline & & S96T002882 & DSC, TGA, TOC, TIC \\
\hline & & S96T002928 & $\overline{\mathrm{IC}}$ \\
\hline & & S96T002913 & ICP-fusion, GEA, total alpha \\
\hline & \multirow{4}{*}{$\begin{array}{l}\text { Segment 4, upper } \\
\text { half }\end{array}$} & S96T002867 & Bulk density \\
\hline & & S96T002881 & DSC, TGA, TOC, TIC \\
\hline & & S96T002927 & IC \\
\hline & & S96T002912 & ICP-fusion, GEA, total alpha \\
\hline & \multirow{4}{*}{$\begin{array}{l}\text { Segment 5, lower } \\
\text { half }\end{array}$} & S96T002870 & Bulk density \\
\hline & & S96T002884 & DSC, TGA, TOC, TIC \\
\hline & & S96T002930 & IC \\
\hline & & S96T002915 & ICP-fusion, GEA, total alpha \\
\hline & \multirow{4}{*}{$\begin{array}{l}\text { Segment 5, upper } \\
\text { half }\end{array}$} & S96T002869 & Bulk density \\
\hline & & S96T002883 & DSC, TGA, TOC, TIC \\
\hline & & S96T002929 & $\mathrm{IC}$ \\
\hline & & S96T002914 & ICP-fusion, GEA, total alpha \\
\hline & \multirow{4}{*}{$\begin{array}{l}\text { Segment } 6 \text {, lower } \\
\text { half }\end{array}$} & S96T002872 & Bulk density \\
\hline & & S96T002886 & DSC, TGA, TOC, TIC \\
\hline & & S96T002932 & IC \\
\hline & & S96T002917 & ICP-fusion, GEA, total alpha \\
\hline & \multirow{4}{*}{$\begin{array}{l}\text { Segment } 6 \text {, upper } \\
\text { half }\end{array}$} & S96T002871 & Bulk density \\
\hline & & S96T002885 & DSC, TGA, TOC, TIC \\
\hline & & S96T002931 & IC \\
\hline & & S96T002916 & ICP-fusion, GEA, total alpha \\
\hline & \multirow{4}{*}{$\begin{array}{l}\text { Segment 7, lower } \\
\text { half }\end{array}$} & S96T002874 & Bulk density \\
\hline & & S96T002888 & DSC, TGA, TOC, TIC \\
\hline & & S96T002934 & IC \\
\hline & & S96T002919 & ICP-fusion, GEA, total alpha \\
\hline
\end{tabular}


Table B1-5. Tank 241-U-103 Sample Analysis Summary. (8 sheets)

\begin{tabular}{|c|c|c|c|}
\hline (.) & Stample Portient & 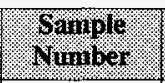 & Aualysis \\
\hline \multirow{25}{*}{$\begin{array}{l}145 \\
\text { (Cont'd) }\end{array}$} & \multirow{4}{*}{$\begin{array}{l}\text { Segment } 7 \text {, upper } \\
\text { half }\end{array}$} & S96T002873 & Bulk density \\
\hline & & S96T002887 & DSC, TGA, TOC, TIC \\
\hline & & S96T002933 & IC \\
\hline & & S96T002918 & ICP-fusion, GEA, total alpha \\
\hline & \multirow{4}{*}{$\begin{array}{l}\text { Segment } 8 \text {, lower } \\
\text { half }\end{array}$} & S96T002876 & Bulk density \\
\hline & & S96T002890 & DSC, TGA, TOC, TIC \\
\hline & & S96T002936 & IC \\
\hline & & S96T002921 & ICP-fusion, GEA, total alpha \\
\hline & \multirow{4}{*}{$\begin{array}{l}\text { Segment } 8 \text {, upper } \\
\text { half }\end{array}$} & S96T002875 & Bulk density \\
\hline & & S96T002935 & IC \\
\hline & & S96T002889 & DSC, TGA, TOC, TIC \\
\hline & & S96T002920 & ICP-fusion, GEA, total alpha \\
\hline & \multirow{3}{*}{$\begin{array}{l}\text { Segment } 9 \text {, lower } \\
\text { half }\end{array}$} & S96T002892 & DSC, TGA, TOC, TIC \\
\hline & & S96T002938 & $\mathrm{IC}$ \\
\hline & & S96T002923 & ICP-fusion, GEA, total alpha \\
\hline & \multirow{4}{*}{$\begin{array}{l}\text { Segment 9, upper } \\
\text { half }\end{array}$} & S96T002877 & Bulk density \\
\hline & & S96T002891 & DSC, TGA, TOC, TIC \\
\hline & & S96T002937 & IC \\
\hline & & S96T002922 & ICP-fusion, GEA, total alpha \\
\hline & \multirow{6}{*}{$\begin{array}{l}\text { Solid core } \\
\text { composite }\end{array}$} & S96T003454 & Bulk density \\
\hline & & S96T003659 & DSC, TGA, TOC, TIC \\
\hline & & S96T003661 & IC \\
\hline & & S96T003457 & ICP-Acid \\
\hline & & S96T003660 & $\begin{array}{l}\text { ICP-fusion, GEA, total uranium, total beta, } \\
\text { Sr-90, total alpha }\end{array}$ \\
\hline & & S96T003662 & ICP-Water \\
\hline \multirow[t]{5}{*}{146} & $\begin{array}{l}\text { Segment } 1 \text {, } \\
\text { drainable liq. }\end{array}$ & S96T003163 & $\begin{array}{l}\text { DSC, TGA, specific gravity, TOC, TIC, ICP, } \\
\text { IC, total alpha }\end{array}$ \\
\hline & \multirow{4}{*}{$\begin{array}{l}\text { Segment } 1 \text {, upper } \\
\text { balf }\end{array}$} & S96T003112 & Bulk density \\
\hline & & S96T003151 & IC \\
\hline & & S96T003121 & DSC, TGA, TOC, TIC \\
\hline & & S96T003143 & ICP-fusion, GEA, total alpha \\
\hline
\end{tabular}


Table B1-5. Tank 241-U-103 Sample Analysis Summary. (8 sheets)

\begin{tabular}{|c|c|c|c|}
\hline 0018 & Somingle Porron & 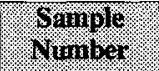 & (1na) \\
\hline \multirow{32}{*}{$\begin{array}{l}146 \\
\text { (Cont'd) }\end{array}$} & \multirow{4}{*}{$\begin{array}{l}\text { Segment } 2 \text {, lower } \\
\text { half }\end{array}$} & S96T003114 & Bulk density \\
\hline & & S96T003124 & DSC, TGA, TOC, TIC \\
\hline & & S96T003153 & $\overline{\mathrm{IC}}$ \\
\hline & & S96T003145 & ICP-fusion, GEA, total alpha \\
\hline & \multirow{4}{*}{$\begin{array}{l}\text { Segment 2, upper } \\
\text { half }\end{array}$} & S96T003113 & Bulk density \\
\hline & & S96T003152 & IC \\
\hline & & S96T003123 & DSC, TGA, TOC, TIC \\
\hline & & S96T003144 & ICP-fusion, GEA, total alpha \\
\hline & \multirow{4}{*}{$\begin{array}{l}\text { Segment 3, lower } \\
\text { half }\end{array}$} & S96T003116 & Bulk density \\
\hline & & S96T003126 & DSC, TGA, TOC, TIC \\
\hline & & S96T003155 & IC \\
\hline & & S96T003147 & ICP-fusion, GEA, total alpha \\
\hline & \multirow{4}{*}{$\begin{array}{l}\text { Segment 3, upper } \\
\text { half }\end{array}$} & S96T003115 & Bulk density \\
\hline & & S96T003154 & IC \\
\hline & & S96T003125 & DSC, TGA, TOC, TIC \\
\hline & & S96T003146 & ICP-fusion, GEA, total alpha \\
\hline & \multirow{4}{*}{$\begin{array}{l}\text { Segment } 3 A \text {, } \\
\text { upper half }\end{array}$} & S96T002950 & Bulk density \\
\hline & & S96T002959 & DSC, TGA, TOC, TIC \\
\hline & & S96T003052 & IC \\
\hline & & S96T003001 & ICP-fusion, GEA, total alpha \\
\hline & \multirow{4}{*}{$\begin{array}{l}\text { Segment } 4 \text {, lower } \\
\text { half }\end{array}$} & S96T002952 & Bulk density \\
\hline & & S96T003054 & IC \\
\hline & & S96T002961 & DSC, TGA, TOC, TIC \\
\hline & & S96T003003 & ICP-fusion, GEA, total alpha \\
\hline & \multirow{4}{*}{$\begin{array}{l}\text { Segment } 4 \text {, upper } \\
\text { half }\end{array}$} & S96T002951 & Bulk density \\
\hline & & S96T003053 & IC \\
\hline & & S96T002960 & DSC, TGA, TOC, TIC \\
\hline & & S96T003002 & ICP-fusion, GEA, total alpha \\
\hline & \multirow{4}{*}{$\begin{array}{l}\text { Segment 5, lower } \\
\text { half }\end{array}$} & S96T002954 & Bulk density \\
\hline & & S96T002963 & DSC, TGA, TOC, TIC \\
\hline & & S96T003056 & IC \\
\hline & & S96T003005 & ICP-fusion, GEA, total alpha \\
\hline
\end{tabular}


HNF-SD-WM-ER-639 Rev. 0

Table B1-5. Tank 241-U-103 Sample Analysis Summary. (8 sheets)

\begin{tabular}{|c|c|c|c|}
\hline (8) & Sample Iortion & $\begin{array}{l}\text { Sample } \\
\text { Yinnber }\end{array}$ & lnavis. \\
\hline \multirow{32}{*}{$\begin{array}{l}146 \\
\text { (Cont'd) }\end{array}$} & \multirow{4}{*}{$\begin{array}{l}\text { Segment } 5, \text { upper } \\
\text { half }\end{array}$} & S96T002953 & Bulk density \\
\hline & & S96T003055 & IC \\
\hline & & S96T002962 & DSC, TGA, TOC, TIC \\
\hline & & S96T003004 & ICP-fusion, GEA, total alpha \\
\hline & \multirow{4}{*}{$\begin{array}{l}\text { Segment 6, lower } \\
\text { half }\end{array}$} & S96T002956 & Bulk density \\
\hline & & S96T002965 & DSC, TGA, TOC, TIC \\
\hline & & S967003058 & IC \\
\hline & & S96T003007 & ICP-fusion, GEA, total alpha \\
\hline & \multirow{4}{*}{$\begin{array}{l}\text { Segment 6, upper } \\
\text { half }\end{array}$} & S96T002955 & Bulk density \\
\hline & & S96T003057 & IC \\
\hline & & S96T002964 & DSC, TGA, TOC, TIC \\
\hline & & S96T003006 & ICP-fusion, GEA, total alpha \\
\hline & \multirow{4}{*}{$\begin{array}{l}\text { Segment } 7 \text {, lower } \\
\text { half }\end{array}$} & S96T002958 & Bulk density \\
\hline & & S96T002967 & DSC, TGA, TOC, TIC \\
\hline & & S96T003060 & IC \\
\hline & & S96T003009 & ICP-fusion, GEA, total alpha \\
\hline & \multirow{4}{*}{$\begin{array}{l}\text { Segment } 7 \text {, upper } \\
\text { half }\end{array}$} & S96T002957 & Bulk density \\
\hline & & S96T003059 & IC \\
\hline & & S96T002966 & DSC, TGA, TOC, TIC \\
\hline & & S96T003008 & ICP-fusion, GEA, total alpha \\
\hline & \multirow{4}{*}{$\begin{array}{l}\text { Segment } 8 \text {, lower } \\
\text { half }\end{array}$} & S96T003118 & Bulk density \\
\hline & & S96T003128 & DSC, TGA, TOC, TIC \\
\hline & & S96T003157 & IC \\
\hline & & S96T003149 & ICP-fusion, GEA, total alpha \\
\hline & \multirow{4}{*}{$\begin{array}{l}\text { Segment } 8, \text { upper } \\
\text { half }\end{array}$} & S96T003117 & Bulk density \\
\hline & & S96T003156 & IC \\
\hline & & S96T003127 & DSC, TGA, TOC, TIC \\
\hline & & S96T003148 & ICP-fusion, GEA, total alpha \\
\hline & \multirow{4}{*}{$\begin{array}{l}\text { Segment } 9 \text {, lower } \\
\text { half }\end{array}$} & S96T003120 & Bulk density \\
\hline & & \$96T004178 & DSC, TGA, TOC, TIC \\
\hline & & S96T004181 & IC \\
\hline & & S96T004180 & ICP-fusion, GEA, total alpha \\
\hline
\end{tabular}


Table B1-5. Tank 241-U-103 Sample Analysis Summary. (8 sheets)

\begin{tabular}{|c|c|c|c|}
\hline Core. & Sample Porrion & $\begin{array}{l}\text { Sample: } \\
\text { Number: }\end{array}$ & Analysis \\
\hline \multirow{10}{*}{$\begin{array}{l}146 \\
\text { (Cont'd) }\end{array}$} & \multirow{4}{*}{$\begin{array}{l}\text { Segment } 9 \text {, upper } \\
\text { half }\end{array}$} & S96T003119 & Bulk density \\
\hline & & S96T003129 & DSC, TGA, TOC, TIC \\
\hline & & S96T003158 & IC \\
\hline & & S96T003150 & ICP-fusion, GEA, total alpha \\
\hline & \multirow[t]{6}{*}{$\begin{array}{l}\text { Solid core } \\
\text { composite }\end{array}$} & S96T004197 & Bulk density \\
\hline & & S96T004198 & DSC, TGA, TOC, TIC \\
\hline & & S96T004201 & IC -2 \\
\hline & & S96T004200 & ICP-Acid \\
\hline & & S96T004199 & $\begin{array}{l}\text { ICP-fusion, GEA, total uranium, total beta, } \\
\text { Sr-90, total alpha }\end{array}$ \\
\hline & & S96T004202 & ICP-Water \\
\hline
\end{tabular}

Note:

$\mathrm{GEA}=$ Gamma energy analysis

\section{B1.4 DESCRIPTION OF 1995 VAPOR SAMPLING}

Tank 241-U-108 headspace gas and vapor samples were collected and analyzed to help determine the potential risks from fugitive emissions to tank farm workers. Analysis of these samples also satisfies the organic solvent screening requirements (Cash 1996). The drivers and objectives of waste tank headspace sampling and analysis are discussed in Program Plan for the Resolution of Tank Vapor Issues (Osborne and Huckaby 1994). Tank 241-U-108 was vapor sampled in accordance with Data Quality Objectives for Generic In-Tank Health and Safety Issue Resolution (Osborne et al. 1995).

Headspace gas and vapor samples were collected from tank 241-U-108 using the Vapor Sampling System (VSS) on August 29, 1995 by Westinghouse Hanford Company (WHC) Sampling and Mobile Laboratories. Sample collection and analyses were performed as directed by the vapor sampling and analysis plan (Homi 1995). Air from the tank 241-U-108 headspace was withdrawn via a heated sampling probe mounted in riser 10 , and transferred via heated tubing to the VSS sampling manifold. All heated zones of the VSS were maintained at approximately $60{ }^{\circ} \mathrm{C}\left(140{ }^{\circ} \mathrm{F}\right)$. Further discussion of the methods used for collection and analysis of the vapor samples can be found in Mahon (1995). 


\section{B1.5 SAMPLE HANDLING AND ANALYSIS (1995 VAPOR SAMPLES)}

Tank 241-U-108 headspace samples were analyzed at Pacific Northwest National Laboratory (PNNL) for inorganic and organic analytes. The analytical work was performed by the PNNL Vapor Analytical Laboratory in the 300 Area of the Hanford Site. Analytical results were reported in Thomas et al. (1996). Sampling devices, sample volumes, analytes, and number of samples are shown in Table B1-6.

Table B1-6. Tank 241-U-108 Gas and Vapor Sample Type and Number for the 1995 Tank 241-U-108 Vapor Samples. ${ }^{1}$

\begin{tabular}{|c|c|c|c|}
\hline 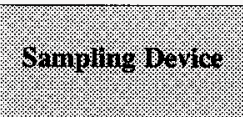 & 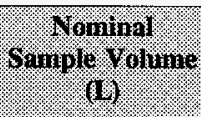 & 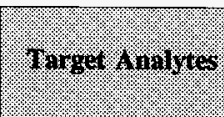 & hinimar. of gamples \\
\hline SUMMA $^{2}$ canister & 6.0 & $\begin{array}{l}\text { Hydrogen, nitrous } \\
\text { oxide, carbon } \\
\text { dioxide, carbon } \\
\text { monoxide }\end{array}$ & $\begin{array}{l}3 \text { tank air samples, } 2 \text { ambient } \\
\text { air blanks, } 3 \text { field blanks }\end{array}$ \\
\hline $\begin{array}{l}\text { Acidified carbon } \\
\text { sorbent trap }\end{array}$ & 3.0 & Ammonia & $\begin{array}{l}6 \text { tank air samples, } 2 \text { trip } \\
\text { blanks }\end{array}$ \\
\hline $\begin{array}{l}\text { Triethanolamine } \\
\text { sorbent trap }\end{array}$ & 3.0 & Nitrogen dioxide & $\begin{array}{l}6 \text { tank air samples, } 2 \text { trip } \\
\text { blanks }\end{array}$ \\
\hline $\begin{array}{l}\text { Oxidation bed plus } \\
\text { triethanolamine } \\
\text { sorbent trap }\end{array}$ & 3.0 & Nitric oxide & $\begin{array}{l}6 \text { tank air samples, } 2 \text { trip } \\
\text { blanks }\end{array}$ \\
\hline $\begin{array}{l}\text { Silica gel sorbent } \\
\text { trap }\end{array}$ & 3.0 & Water vapor & $\begin{array}{l}8 \text { tank air samples, } 2 \text { trip } \\
\text { blanks }\end{array}$ \\
\hline SUMMA $^{\mathrm{TM}}$ canister & 6.0 & $\begin{array}{l}\text { Organic vapors } \\
\text { (including total } \\
\text { non-methane } \\
\text { hydrocarbons) }\end{array}$ & $\begin{array}{l}3 \text { tank air samples, } 2 \text { ambient } \\
\text { air samples, } 3 \text { field blanks }\end{array}$ \\
\hline Triple sorbent trap & 1.0 & $\begin{array}{l}\text { Semi-volatile } \\
\text { organic vapors }\end{array}$ & $\begin{array}{l}6 \text { tank air samples, } 6 \text { field } \\
\text { blanks, } 2 \text { trip blanks }\end{array}$ \\
\hline
\end{tabular}

Notes:

'Thomas et al. (1996)

${ }^{2}$ SUMMA is a trademark of Molectrics, Inc., Cleveland, Ohio. 
Sorbent traps for inorganic analytes were either weighed (for water analysis) or weighed and desorbed with the appropriate aqueous solutions for analyzing inorganic analytes by either ion-selective electrode or ion chromatography. Triple sorbent traps were used for semi-volatile organic analytes by gas chromatography/mass spectroscopy. Tank headspace samples were also analyzed for permanent gases (hydrogen, nitrous oxide, carbon dioxide, and carbon monoxide) by gas chromatography (GC), total non-methane hydrocarbons using cryogenic preconcentration followed by $\mathrm{GC}$, and volatile organic analysis using cryogenic preconcentration followed by GC.

\section{B1.6 HISTORICAL SAMPLING EVENTS}

Historical analytical data are available for tank 241-U-108's sampling events. Data from waste samples obtained from the tank in 1971, 1973 or 1974, and 1975 were taken from Atlantic Richfield Hanford Company internal memoranda. The analytical results are presented in Section B2.0. Because of the uncertainty in sampling locations and analytical procedures, and the age of some of these data, these historical data were not used to assess the current contents or the status of the tank with respect to safety. Furthermore, pre-1989 analytical data have not been validated and should be used with caution.

\section{B1.6.1 Description of Historical Sampling Events}

Samples were removed from tank 241-U-108 in 1971 (Puryear 1971) and in late 1973 or early 1974 (Sant 1974) in order to identify feed material for the 242-S Evaporator. It is not clear whether the 1971 sample was solid or liquid. The 1973 or 1974 sample was identified as clear and yellow with no solids. A sludge sample was taken and analyzed in 1975 because tank 241-U-108 was being considered as a slurry receiving tank for 242-S Evaporator waste at that time (Horton 1975). Sampling dates and locations were not provided for any of the three sampling events. It appears the analyses were performed at the 222-S Laboratory, although the above references are not clear in that regard.

\section{B2.0 ANALYTICAL RESULTS}

\section{B2.1 OVERVIEW}

This section summarizes the sampling and analytical results associated with the 1996 core, and the 1995 grab and vapor samples from tank 241-U-108. The location of the analytical results associated with this tank are presented in Table B2-1. The core sampling results were documented in Bell (1996a), the grab sampling results were documented in Esch (1995), and the vapor results are reported in Thomas et al. (1996). 
Table B2-1. Analytical Presentation Tables.

\begin{tabular}{|l|l|}
\hline \multirow{2}{*}{ Percent water by TGA } & \multicolumn{1}{|c|}{ Table Number } \\
\hline DSC & B2-2, B2-3 \\
\hline Bulk density & B2-4, B2-5 \\
\hline Specific gravity & B2-6 \\
\hline pH & B2-7, B2-8 \\
\hline TOC & B2-9 \\
\hline TIC & B2-10, B2-11 \\
\hline Hydroxide & B2-12, B2-13 \\
\hline Summary data for metals by ICP & B2-14 \\
\hline Anions by IC & B2-16 through B2-37 \\
\hline Total alpha activity & B2-38 through B2-50 \\
\hline Sr-90 & B2-51 \\
\hline Total beta & B2-52, B2-53 \\
\hline Total uranium & B2-54 \\
\hline Pu-239/240 & B2-55 \\
\hline Am-241 & B2-56 \\
\hline Radionuclides by GEA & B2-57 \\
\hline 1995 vapor results summary & B2-58 through B2-64 \\
\hline Flammable gas monitoring & B2-65 \\
\hline
\end{tabular}

The four QC parameters assessed in conjunction with the tank 241-U-108 samples were standard recoveries, spike recoveries, duplicate analyses (relative percent difference [RPDs]), and blanks. The QC criteria specified in the core SAP (Homi 1996) were either 80 to 120 or 90 to 110 percent recovery for standards and spikes, and $\leq 20$ or $\leq 10$ percent for RPDs, depending on the analyte. The QC criteria specified in the grab SAP (Schreiber 1995) were 80 to 120 percent recovery for standards and spikes and $\leq 20$ percent for RPDs. The only QC parameter for which limits are not specified in the core and grab SAPs is blank contamination. The limits for blanks are set forth in guidelines followed by the laboratory, and all data results presented in this report have met those guidelines. The QC criteria for the 1995 vapor samples are discussed in Section B2.5. 
Sample and duplicate pairs in which any of the QC parameters were outside of these limits are footnoted in the sample mean column of the following data summary tables with an $a, b$, $c, d$, or e as follows:

- "a" indicates that the standard recovery was below the QC limit.

- " $b$ " indicates that the standard recovery was above the QC limit.

- "c" indicates that the spike recovery was below the QC limit.

- "d" indicates that the spike recovery was above the QC limit.

- " $\mathrm{e}$ " indicates that the RPD was above the QC limit.

- "f" indicates that there was blank contamination.

In the analytical tables in this section, the "Mean" column is the average of the result and duplicate values. All values, including those below the detection level (denoted by the less-than symbol, "<"), were averaged. If both sample and duplicate values were non-detected, the mean is expressed as a non-detected value. If one value was detected while the other was not, the mean is expressed as a detected value. If both values were detected, the mean is expressed as a detected value.

\section{B2.2 THERMODYNAMIC ANALYSES}

As required by the safety screening, organic, historical, and compatibility DQOs, and the organic test plan, analysis by TGA and DSC was performed on all samples. Other physical tests required were bulk density (Section B2.3.1), specific gravity (Section B2.3.2), and pH (Section B2.3.3).

\section{B2.2.1 Thermogravimetric Analysis}

Thermogravimetric analysis measures the mass of a sample while its temperature is increased at a constant rate. Nitrogen is passed over the sample during heating to remove any released gases. Any decrease in the weight of a sample during TGA represents a loss of gaseous matter from the sample, either through evaporation or through a reaction that forms gas phase products. The moisture content is estimated by assuming all TGA sample weight loss up to a certain temperature (typically 150 to $200^{\circ} \mathrm{C}\left[300\right.$ to $390^{\circ} \mathrm{F}$ ) is caused by water evaporation. The temperature limit for moisture loss is chosen by the operator at an inflection point on the TGA plot. Other volatile matter fractions can often be differentiated by inflection points as well. 
Analyses for percent water by TGA were performed on the core samples using procedures LA-560-112, Rev. B-1 (Mettler ${ }^{1}$ instrument) and LA-514-114, Rev. C-1 (Perkin-Elmer ${ }^{2}$ instrument). Grab sample U-108-1 was analyzed by procedure LA-560-112, Rev. A-2. All samples were run under a nitrogen atmosphere. The TGA results for the tank 241-U-108 samples are summarized in Tables B2-2 and B2-3.

Although the core SAP (Homi 1996) indicates that there is no notification limit for moisture by TGA, the organic DQO does have a notification limit of $<17$ percent. Only the sample from the lower half of segment 2 of core 146 had an average moisture value below 17 percent $(12.27$ percent). However, the TOC content of that sample was far below 3 percent $(0.174$ percent) and the sample had a small exotherm $(69 \mathrm{~J} / \mathrm{g})$.

Table B2-2. Tank 241-U-108 Core Sample Analytical Results: Percent Water (TGA). (3 sheets)

\begin{tabular}{|c|c|c|c|c|c|c|}
\hline Samples & Sormple & $\begin{array}{l}\text { Sirriple } \\
\text { rortion }\end{array}$ & Rernit & Dilulicate & Triplicare: & Merin: \\
\hline \multicolumn{2}{|c|}{ :- } & & 1.96 & 1.92 & 96 & 8.8 \\
\hline S96T002241 & 141: 1 & Lower $1 / 2$ & 45.92 & 45.08 & & 45.5 \\
\hline S96T002242 & 141: 2 & Upper $1 / 2$ & 23.05 & 24.43 & & 23.74 \\
\hline S96T002243 & $141: 3$ & Upper $1 / 2$ & 33.54 & 26.98 & & $30.26^{\mathrm{QC}: \mathrm{e}}$ \\
\hline S96T002244 & $141: 3 A$ & Upper $1 / 2$ & $19 . \overline{44}$ & 32.04 & & $25.74^{\mathrm{QC}: \mathrm{e}}$ \\
\hline S96T002245 & \multirow[t]{2}{*}{ 141: 4} & Upper $1 / 2$ & 33.04 & 32.57 & & 32.805 \\
\hline S96T002246 & & Lower $1 / 2$ & 28.59 & 31.19 & & 29.89 \\
\hline S96T002247 & \multirow[t]{2}{*}{ 141: 4A } & Upper $1 / 2$ & 0.986 & 44.42 & 44.53 & $29.979^{\mathrm{QC}: \mathrm{e}}$ \\
\hline S96T002248 & & Lower $1 / 2$ & 39.46 & 38.73 & & 39.095 \\
\hline S96T002585 & \multirow[t]{2}{*}{$141: 5$} & Upper $1 / 2$ & 24.85 & 29.17 & & $27.01^{\mathrm{QC}: \mathrm{e}}$ \\
\hline S96T002586 & & Lower $1 / 2$ & 28.40 & 35.74 & & $32.07^{\mathrm{QC}: \mathrm{e}}$ \\
\hline S96T002587 & \multirow[t]{2}{*}{$141: 6$} & Upper $1 / 2$ & 23.06 & 21.32 & & 22.19 \\
\hline S96T002588 & & Lower $1 / 2$ & 21.21 & 15.68 & & $18.445^{\mathrm{QC}: e}$ \\
\hline S96T002589 & \multirow[t]{2}{*}{$141: 7$} & Upper $1 / 2$ & 39.52 & 38.20 & & 38.86 \\
\hline S96T002590 & & Lower $1 / 2$ & 40.30 & 40.15 & & 40.225 \\
\hline S96T002591 & \multirow[t]{2}{*}{$141: 8$} & Upper $1 / 2$ & 38.04 & 42.06 & 42.04 & $40.7133^{\mathrm{QC}: \mathrm{e}}$ \\
\hline S96T002592 & & Lower $1 / 2$ & 41.31 & 37.90 & & 39.605 \\
\hline
\end{tabular}

\footnotetext{
${ }^{1}$ Mettler is a trademark of Mettler Electronics, Anaheim, California.

${ }^{2}$ Perkin-Elmer is a trademark of Perkins Research and Manufacturing Company, Inc., Canoga Park, California.
} 
Table B2-2. Tank 241-U-108 Core Sample Analytical Results: Percent Water (TGA). (3 sheets)

\begin{tabular}{|c|c|c|c|c|c|c|}
\hline $\begin{array}{l}\text { Sanple } \\
\text { Iumiler }\end{array}$ & $\begin{array}{l}\text { Gample } \\
\text { Eqcation }\end{array}$ & $\begin{array}{l}\text { Sample } \\
\text { Eortion. }\end{array}$ & Resulit & Buplinate & Tribilicate & Meari \\
\hline \multicolumn{2}{|c|}{ 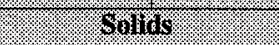 } & 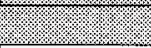 & 9. & 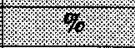 & 9.8 & \%: 80 \\
\hline S96T002593 & $141: 9$ & Upper $1 / 2$ & 30.76 & 33.88 & & 32.32 \\
\hline S96T002893 & $145: 2$ & Upper $1 / 2$ & 34.42 & 31.90 & & 33.16 \\
\hline S96T002879 & \multirow[t]{2}{*}{$145: 3$} & Upper $1 / 2$ & 69.61 & 47.71 & 39.27 & $52.1967^{\mathrm{QC}: \mathrm{e}}$ \\
\hline S96T002880 & & Lower $1 / 2$ & 45.56 & 45.98 & & 45.77 \\
\hline S96T002881 & \multirow[t]{2}{*}{$145: 4$} & Upper $1 / 2$ & 41.97 & 39.96 & & 40.965 \\
\hline S96T002882 & & Lower $1 / 2$ & 25.88 & 25.56 & & 25.72 \\
\hline S96T002883 & \multirow[t]{2}{*}{ 145: 5} & Upper $1 / 2$ & 18.15 & 17.30 & & $17 . \overline{725}$ \\
\hline S96T002884 & & Lower $1 / 2$ & 28.41 & 33.46 & & $30.935^{\mathrm{oC} e}$ \\
\hline S96T002885 & \multirow[t]{2}{*}{$145: 6$} & Upper $1 / 2$ & 28.67 & 27.50 & & 28.085 \\
\hline S96T002886 & & Lower $1 / 2$ & 29.39 & 38.89 & & $34.14^{\mathrm{QC}: \mathrm{e}}$ \\
\hline S96T002887 & \multirow[t]{2}{*}{$145: 7$} & Upper $1 / 2$ & 45.85 & 42.45 & & 44.15 \\
\hline S96T002888 & & Lower $1 / 2$ & 42.33 & 42.24 & & 42.285 \\
\hline S96T002889 & \multirow[t]{2}{*}{$145: 8$} & Upper $1 / 2$ & 41.50 & 42.92 & & 42.21 \\
\hline S96T002890 & & Lower $1 / 2$ & 41.17 & 40.04 & & 40.605 \\
\hline S96T002891 & \multirow[t]{2}{*}{$145: 9$} & Upper $1 / 2$ & 40.10 & 38.34 & & 39.22 \\
\hline S96T002892 & & Lower $1 / 2$ & 48.86 & 49.67 & & 49.265 \\
\hline S96T003121 & 146: 1 & Upper $1 / 2$ & 41.79 & 37.70 & & $39.745^{\text {QC:e }}$ \\
\hline S96T003123 & \multirow[t]{2}{*}{ 146: 2} & Upper $1 / 2$ & 29.51 & 23.66 & & $26.585^{\mathrm{QC}: e}$ \\
\hline S96T003124 & & Lower $1 / 2$ & 9.59 & 14.94 & & $12.265^{\mathrm{QC}: \mathrm{e}}$ \\
\hline S96T003125 & \multirow[t]{2}{*}{ 146: 3} & Upper $1 / 2$ & 29.49 & 41.56 & & $35.525^{\mathrm{QC}: \mathrm{e}}$ \\
\hline S96T003126 & & Lower $1 / 2$ & 19.20 & 21.63 & & $20.415^{\mathrm{QC}: \mathrm{e}}$ \\
\hline S96T002959 & 146: $3 \mathrm{~A}$ & Upper $1 / 2$ & 17.24 & 23.12 & & $20.18^{\mathrm{QC}: \mathrm{e}}$ \\
\hline S96T002960 & 146: 4 & Upper $1 / 2$ & 17.89 & 17.21 & & 17.55 \\
\hline S96T002962 & \multirow[t]{2}{*}{ 146: 5} & Upper $1 / 2$ & 41.13 & 36.72 & & $38.925^{\text {QC:e }}$ \\
\hline S96T002963 & & Lower $1 / 2$ & 38.38 & 31.17 & & $34.775^{\text {QC:e }}$ \\
\hline S96T002964 & \multirow[t]{2}{*}{ 146: 6} & Upper $1 / 2$ & 29.48 & 35.35 & & $32.415^{\mathrm{QC}: \mathrm{e}}$ \\
\hline S96T002965 & & Lower $1 / 2$ & 44.30 & 45.61 & & 44.955 \\
\hline S96T002966 & \multirow[t]{2}{*}{ 146: 7} & Upper $1 / 2$ & 33.55 & 33.30 & & 33.425 \\
\hline S96T002967 & & Lower $1 / 2$ & 41.14 & 40.96 & & 41.05 \\
\hline S96T003127 & \multirow[t]{2}{*}{$146: 8$} & Upper $1 / 2$ & 41.25 & 41.28 & & 41.265 \\
\hline S96T003128 & & Lower $1 / 2$ & 40.39 & 40.07 & & 40.23 \\
\hline
\end{tabular}


Table B2-2. Tank 241-U-108 Core Sample Analytical Results: Percent Water (TGA). (3 sheets)

\begin{tabular}{|c|c|c|c|c|c|c|}
\hline $\begin{array}{l}\text { Sample: } \\
\text { Inminer }\end{array}$ & $\begin{array}{l}\text { Sample } \\
\text { I Gucation }\end{array}$ & $\begin{array}{l}\text { Sample } \\
\text { Iortion }\end{array}$ & resiliti & Binpiluate & Truplicate & Mran \\
\hline \multicolumn{2}{|c|}{ SWits: } & 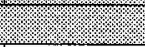 & 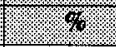 & 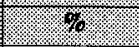 & \% & 9.96 \\
\hline S96T004178 & \multirow[t]{2}{*}{ 146: 9} & Lower $1 / 2$ & 48.59 & 47.83 & & 48.21 \\
\hline S96T003129 & & \begin{tabular}{|l|} 
Upper $1 / 2$ \\
\end{tabular} & 38.62 & 38.46 & & 38.54 \\
\hline S96T003449 & Core 141 & $\begin{array}{l}\text { Solid } \\
\text { composite }\end{array}$ & 30.82 & 40.19 & & $35.505^{\mathrm{QC}: \mathrm{e}}$ \\
\hline S96T003659 & Core 145 & $\begin{array}{l}\text { Solid } \\
\text { composite }\end{array}$ & 32.60 & 29.12 & & $30.86^{\mathrm{QC}: \mathrm{e}}$ \\
\hline S96T004198 & Core 146 & $\begin{array}{l}\text { Solid } \\
\text { composite }\end{array}$ & 28.00 & 28.35 & & 28.175 \\
\hline \multicolumn{2}{|c|}{ Wiquiris } & Y & 96 & $1 \%$ & (6) & 4 \\
\hline S96T002282 & $141: 1$ & $\begin{array}{l}\text { Drainable } \\
\text { liquid }\end{array}$ & 51.08 & 47.33 & & 49.205 \\
\hline S96T002942 & 145: 1 & $\begin{array}{l}\text { Drainable } \\
\text { liquid }\end{array}$ & 49.25 & 49.66 & & 49.455 \\
\hline S96T003163 & $146: 1$ & $\begin{array}{l}\text { Drainable } \\
\text { liquid }\end{array}$ & 51.15 & 51.11 & & 51.13 \\
\hline
\end{tabular}

Table B2-3. Tank 241-U-108 Grab Sample Analytical Results: Percent Water (TGA).

\begin{tabular}{|c|c|c|c|c|c|}
\hline $\begin{array}{l}\text { Sumplo. } \\
\text { trimber }\end{array}$ & 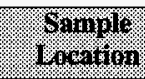 & $\begin{array}{l}\text { Somple: } \\
\text { Portion }\end{array}$ & Mesuli & Diplieare & Mean \\
\hline \multicolumn{2}{|c|}{ Tlquars } & & $\% 8$ & 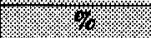 & 9 \\
\hline S95T000978 & Riser 7 & Grab sample & 50.20 & 50.35 & 50.275 \\
\hline
\end{tabular}

\section{B2.2.2 Differential Scanning Calorimetry}

In a DSC analysis, heat absorbed or emitted by a sample is measured while the temperature of the sample is heated at a constant rate. Nitrogen is passed over the sample material to remove any gases being released. The onset temperature for an endothermic or exothermic event is determined graphically.

Analyses by DSC were performed on all 1996 core subsegments and drainable liquids, and the 1995 grab sample from tank 241-U-108. The core sample analyses were performed under a nitrogen purge using procedures LA-514-113, Rev. C-1 (Mettler ${ }^{\mathrm{TM}}$ instrument) and LA-514-114, Rev. C-1 (Perkin-Elmer ${ }^{\mathrm{TM}}$ instrument). The grab sample was analyzed per 
procedure LA-514-113, Rev. B-1. Because two instruments were used during the DSC analyses, it must be noted that each instrument produces raw data scans that present exotherms differently. On the Mettler ${ }^{\mathrm{TM}}$ instrument, an exotherm is represented by a peak, while the Perkin-Elmer ${ }^{\mathrm{TM}}$ instrument shows an exotherm as a valley. Results are presented in Tables B2-4 and B2-5. Only samples that showed or had exotherms were included in the tables. Most core samples displayed exothermic behavior, and one sample from the upper half of segment 4 from core 141 had an average DSC result of $496 \mathrm{~J} / \mathrm{g}$ on a dry-weight basis, thus exceeding the DSC notification limit specified in the SAP. Two other samples exceeded the DSC notification limit at the one-sided 95 percent upper confidence limit of the mean, although the individual measurements did not exceed the $480-\mathrm{J} / \mathrm{g}$ notification limit. The upper limits are provided in Table C1-2.

Table B2-4. Tank 241-U-108 Core Sample Analytical Results: Exotherm Transition 1 (DSC). (2 sheets)

\begin{tabular}{|c|c|c|c|c|c|c|}
\hline Sample & $\begin{array}{l}\text { Saraplo } \\
\text { Socutina }\end{array}$ & $\begin{array}{l}\text { Saripple: } \\
\text { Portion }\end{array}$ & irsinili & Byplicato & Triplicate & Ken \\
\hline \multicolumn{2}{|c|}{ Solids: } & & $\sqrt{118}$ & 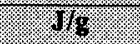 & 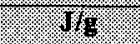 & 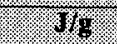 \\
\hline S96T002241 & $141: 1$ & Lower $1 / 2$ & 85.2 & 110.9 & & $98.1^{\mathrm{QC:e}}$ \\
\hline S96T002242 & 141: 2 & Upper $1 / 2$ & 47.8 & 45.4 & & 46.6 \\
\hline S96T002245 & \multirow[t]{3}{*}{$141: 4$} & Upper $1 / 2$ & 408.9 & 257.3 & & $333.1^{\mathrm{QC}: \mathrm{e}}$ \\
\hline S96T002245 & & Upper $1 / 2$ & 67.0 & 44.0 & & $55.5^{\mathrm{QC}: \mathrm{e}}$ \\
\hline S96T002246 & & Lower $1 / 2$ & 232.3 & 74.4 & 159.7 & $155.5^{\mathrm{QC}: \mathrm{e}}$ \\
\hline S96T002589 & \multirow[t]{2}{*}{ 141: 7} & Upper $1 / 2$ & 76.4 & 36.5 & & $56.5^{\mathrm{QC}: \mathrm{e}}$ \\
\hline S96T002590 & & Lower $1 / 2$ & 184.4 & 68.6 & & $126.5^{\mathrm{QC:e}}$ \\
\hline S96T002591 & \multirow[t]{2}{*}{ 141: 8} & Upper $1 / 2$ & 77.4 & 89.2 & 96.7 & $87.8^{\mathrm{QCe}}$ \\
\hline S96T002592 & & Lower $1 / 2$ & 71.9 & 81.9 & & $76.9^{\mathrm{QC:c}}$ \\
\hline S96T002879 & \multirow[t]{2}{*}{$145: 3$} & Upper $1 / 2$ & 86.7 & 99.8 & & $93.2^{\mathrm{oC}: \mathrm{e}}$ \\
\hline S96T002880 & & Lower $1 / 2$ & 88.5 & 89.6 & & 89.1 \\
\hline S96T002881 & 145: 4 & Upper $1 / 2$ & 90.1 & 58.2 & 84.1 & $77.5^{\mathrm{QC:e}}$ \\
\hline S96T002884 & $145: 5$ & Lower $1 / 2$ & 21.4 & 18.1 & & $19.7^{\mathrm{QC:e}}$ \\
\hline S96T002885 & \multirow[t]{2}{*}{$145: 6$} & Upper $1 / 2$ & 26.2 & 80.3 & & $53.3^{\mathrm{QC}: \mathrm{e}}$ \\
\hline S96T002886 & & Lower $1 / 2$ & 153.5 & 0.0 & & $76.7^{\mathrm{QC:e}}$ \\
\hline S96T002887 & \multirow[t]{2}{*}{$145: 7$} & Upper $1 / 2$ & 141.3 & 72.8 & & $107.1^{\mathrm{QC}: \mathrm{e}}$ \\
\hline S96T002888 & & Lower $1 / 2$ & 97.2 & 98.7 & & 97.9 \\
\hline S96T002891 & \multirow[t]{2}{*}{$145: 9$} & Upper $1 / 2$ & 67.8 & 63.6 & & 65.7 \\
\hline S96T002892 & & Lower $1 / 2$ & 52.7 & 22.5 & & $37.6^{\mathrm{QC:e}}$ \\
\hline S96T003121 & $146: 1$ & Upper $1 / 2$ & 65.6 & 71.5 & & 68.6 \\
\hline S96T003124 & 146: 2 & Lower $1 / 2$ & 77.4 & 60.6 & & $69.0^{\mathrm{QC}: \mathrm{e}}$ \\
\hline
\end{tabular}


HNF-SD-WM-ER-639 Rev. 0

Table B2-4. Tank 241-U-108 Core Sample Analytical Results: Exotherm Transition 1 (DSC). (2 sheets)

\begin{tabular}{|c|c|c|c|c|c|c|}
\hline $\begin{array}{l}\text { Gample } \\
\text { Sumber. }\end{array}$ & $\begin{array}{l}\text { Sanple } \\
\text { tercation }\end{array}$ & $\begin{array}{l}\text { Sample. } \\
\text { rortion }\end{array}$ & Resillt & Bripligate & Troliteate & Mean \\
\hline \multicolumn{2}{|c|}{ 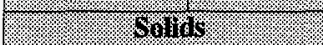 } & (2. & $\sqrt{88}$ & $\sqrt{1 / 9}=$ & $\frac{1}{168}=$ & $\sqrt{18}$ \\
\hline S96T003125 & \multirow[t]{2}{*}{$146: 3$} & Upper $1 / 2$ & 45.9 & 11.1 & & $28.5^{\mathrm{QC:e}}$ \\
\hline S96T003126 & & Lower $1 / 2$ & 3.70 & 0.0 & & 3.70 \\
\hline S96T002959 & 146: $3 \mathrm{~A}$ & Upper $1 / 2$ & $0 . \overline{0}$ & 0.0 & 22.9 & $7.63^{\mathrm{QC:e}}$ \\
\hline S96T002960 & $146: 4$ & Upper $1 / 2$ & 34.1 & 0 & & $17.1^{\mathrm{QC:e}}$ \\
\hline S96T002961 & 146: 4 & Lower $1 / 2$ & 62.4 & $60 . \overline{5}$ & & 61.5 \\
\hline S96T002962 & \multirow[t]{2}{*}{$146: 5$} & Upper $1 / 2$ & 96.9 & $\overline{92.1}$ & & 94.5 \\
\hline S96T002963 & & Lower $1 / 2$ & 101.6 & 87.5 & & $94 . \overline{0^{\mathrm{CC}: \mathrm{e}}}$ \\
\hline S96T002964 & 146: 6 & Upper $1 / 2$ & 30.3 & 18.5 & & $24.4^{\overline{Q C: e}}$ \\
\hline S96T002967 & 146: 7 & Lower $1 / 2$ & 64.5 & 73.3 & & $68.9^{\overline{Q C: e}}$ \\
\hline S96T004178 & \multirow[t]{2}{*}{ 146: 9} & Lower $1 / 2$ & 26.1 & 37.42 & & $31.8^{\mathrm{QC:e}}$ \\
\hline S96T003129 & & Upper $1 / 2$ & 55.6 & 57.6 & & $\overline{56.6}$ \\
\hline S96T003449 & Core 141 & $\begin{array}{l}\text { Solid } \\
\text { composite }\end{array}$ & 76.8 & 110.4 & & $93.6^{\text {QC:e }}$ \\
\hline \multicolumn{2}{|c|}{. Equids } & (1) & 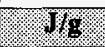 & $1 / 8$ & $1 / 8$ & 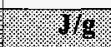 \\
\hline S96T002942 & 145: 1 & $\begin{array}{l}\text { Drainable } \\
\text { liquid }\end{array}$ & 78.3 & 79.5 & & 78.9 \\
\hline S96T003163 & 146: 1 & $\begin{array}{l}\text { Drainable } \\
\text { liquid }\end{array}$ & 77.9 & 88.8 & & $83.3^{\mathrm{QCe} e}$ \\
\hline
\end{tabular}

Table B2-5. Tank 241-U-108 Grab Sample Analytical Results: Exotherm - Transition 1.

\begin{tabular}{|c|c|c|c|c|c|}
\hline Somple. & Somple? & $\begin{array}{l}\text { Samplo: } \\
\text { Fortion: }\end{array}$ & Reswif & Doplleare & Mean \\
\hline & & & T/p & J/g: & T/8 \\
\hline S95T000978 & Riser 7 & Grab sample & 64.2 & 66.7 & 65.45 \\
\hline
\end{tabular}




\section{B2.3 PHYSICAL MEASUREMENTS}

\section{B2.3.1 Bulk Density}

Bulk density was determined on all solid subsamples from cores 141,145 , and 146 except the upper half of segment 9 from core 141, according to procedure LO-160-103, Rev. B-0. The sample from segment 9 of core 141 was a dry, clay-like material. An accurate volume measurement could not be made using the bulk density procedure.

Results shown in Table B2-6 were consistent from core to core and segment to segment. The highest measured bulk density was $2.1 \mathrm{~g} / \mathrm{mL}$ (upper half of segment 2 , core 145 ) and the lowest result was $1.57 \mathrm{~g} / \mathrm{mL}$ (lower half of segment 1 , core 141 ). No duplicate analyses were performed as part of the bulk density measurements.

Table B2-6. Tank 241-U-108 Core Sample Analytical Results: Bulk Density. (2 sheets)

\begin{tabular}{|c|c|c|c|c|}
\hline $\begin{array}{l}\text { Grmple } \\
\text { Nrmaler }\end{array}$ & 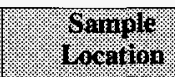 & Geriole & Revali & Mean \\
\hline \multicolumn{3}{|c|}{ Sollos } & 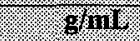 & 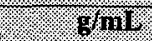 \\
\hline S96T002230 & 141: 1 & Lower $1 / 2$ & 1.57 & 1.57 \\
\hline S96T002231 & 141:2 & Upper $1 / 2$ & 1.75 & 1.75 \\
\hline S96T002232 & $141: 3$ & Upper $1 / 2$ & 1.74 & 1.74 \\
\hline S96T002233 & $141: 3 A$ & Upper $1 / 2$ & 1.77 & 1.77 \\
\hline$\overline{\text { S96T002234 }}$ & \multirow[t]{2}{*}{$141: 4$} & Upper $1 / 2$ & 1.69 & 1.69 \\
\hline S96T002235 & & Lower $1 / 2$ & 1.68 & 1.68 \\
\hline S96T002236 & \multirow[t]{2}{*}{ 141: $4 \mathrm{~A}$} & Upper $1 / 2$ & 1.67 & 1.67 \\
\hline S96T002237 & & Lower $1 / 2$ & 1.69 & 1.69 \\
\hline S96T002562 & \multirow[t]{2}{*}{$141: 5$} & Upper $1 / 2$ & 1.66 & 1.66 \\
\hline S96T002563 & & Lower $1 / 2$ & 1.71 & $1 . \overline{71}$ \\
\hline S96T002564 & \multirow[t]{2}{*}{ 141: 6} & Upper $1 / 2$ & 1.75 & 1.75 \\
\hline S96T002565 & & Lower $1 / 2$ & 1.76 & 1.76 \\
\hline S96T002566 & \multirow[t]{2}{*}{ 141: 7} & Upper $1 / 2$ & $1 . \overline{66}$ & 1.66 \\
\hline S96T002567 & & Lower $1 / 2$ & 1.63 & 1.63 \\
\hline S96T002568 & \multirow[t]{2}{*}{ 141: 8} & Upper $1 / 2$ & 1.61 & 1.61 \\
\hline S96T002569 & & Lower $1 / 2$ & 1.63 & $1 . \overline{63}$ \\
\hline S96T002864 & $145: 2$ & Upper $1 / 2$ & 2.1 & 2.1 \\
\hline S96T002865 & \multirow[t]{2}{*}{$145: 3$} & Upper $1 / 2$ & 1.85 & 1.85 \\
\hline S96T002866 & & Lower $1 / 2$ & 1.85 & 1.85 \\
\hline S96T002867 & \multirow[t]{2}{*}{$145: 4$} & Upper $1 / 2$ & 1.7 & 1.7 \\
\hline S96T002868 & & Lower $1 / 2$ & 1.71 & 1.71 \\
\hline
\end{tabular}


HNF-SD-WM-ER-639 Rev. 0

Table B2-6. Tank 241-U-108 Core Sample Analytical Results: Bulk Density. (2 sheets)

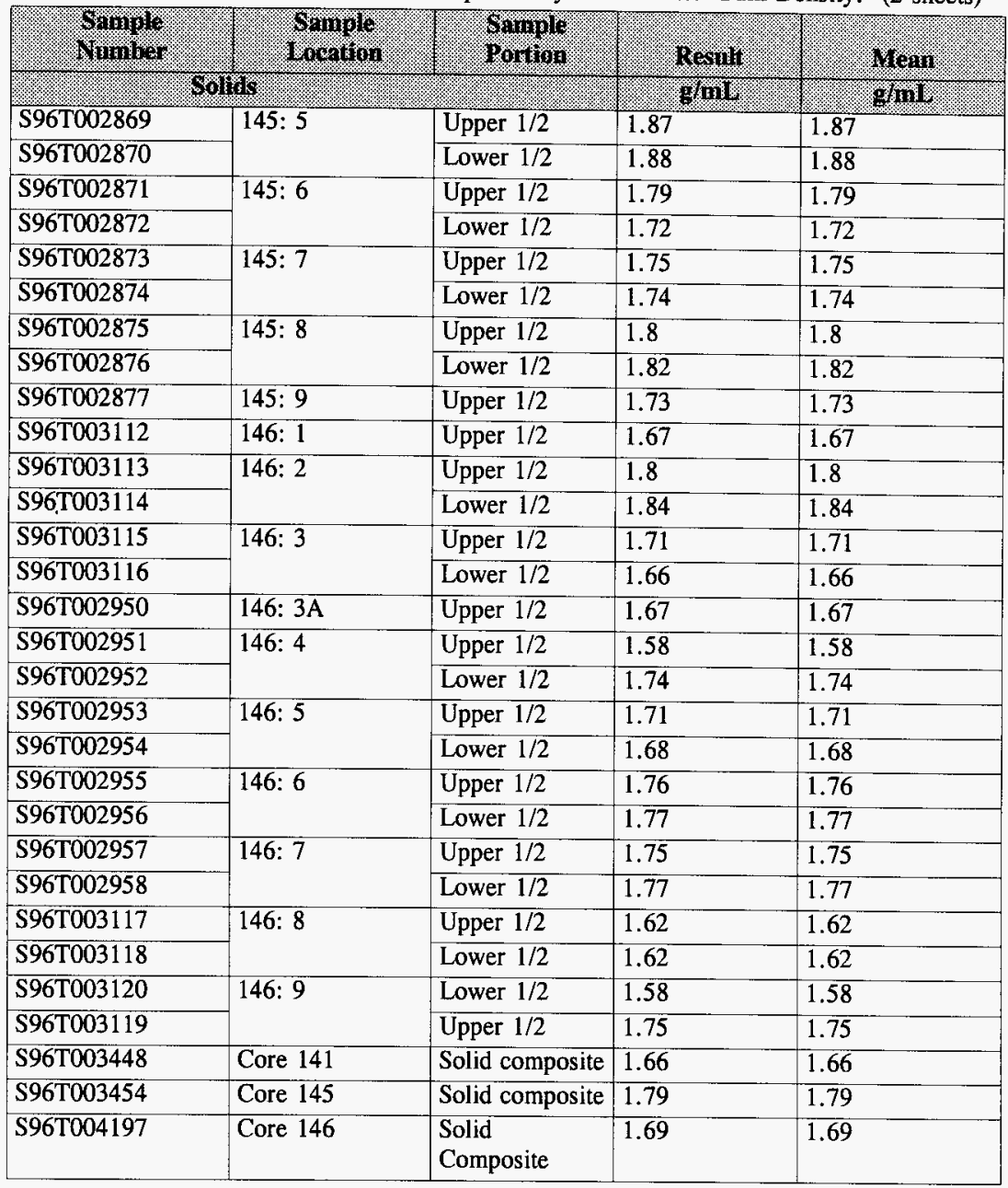




\section{B2.3.2 Specific Gravity}

Specific gravity was determined on the segment 1 drainable liquid from each 1996 core sample, according to procedure LA-510-112, Rev. C-3, and on the one 1995 grab sample per procedure LA-510-112, Rev. C-3. Sample results are shown in Tables B2-7 and B2-8 and varied from 1.38 (core 146) to 1.67 (core 141).

Table B2-7. Tank 241-U-108 Analytical Results: Specific Gravity.

\begin{tabular}{|c|c|c|c|c|c|}
\hline $\begin{array}{l}\text { Sample } \\
\text { Muniner. }\end{array}$ & 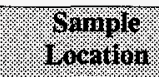 & $\begin{array}{l}\text { Sample } \\
\text { Portion }\end{array}$ & Result & Duplicate & Mean \\
\hline \multicolumn{2}{|c|}{ 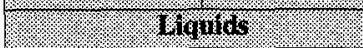 } & 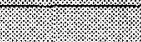 & initiless & 1010110 ss & mintess \\
\hline S96T002282 & 141: 1 & $\begin{array}{l}\text { Drainable } \\
\text { liquid }\end{array}$ & 1.448 & 1.424 & 1.436 \\
\hline S96T002942 & 145: 1 & $\begin{array}{l}\text { Drainable } \\
\text { liquid }\end{array}$ & 1.384 & 1.388 & 1.386 \\
\hline S96T003163 & $146: 1$ & $\begin{array}{l}\text { Drainable } \\
\text { liquid }\end{array}$ & 1.371 & 1.397 & 1.384 \\
\hline
\end{tabular}

Table B2-8. Tank 241-U-108 Grab Sample Analytical Results: Specific Gravity.

\begin{tabular}{|c|c|c|c|c|c|}
\hline $\begin{array}{l}\text { Sanple. } \\
\text { Trunlor. }\end{array}$ & $\begin{array}{l}\text { Ganiple } \\
\text { location }\end{array}$ & $\begin{array}{l}\text { Sampio: } \\
\text { Portion. }\end{array}$ & Rrosull & Duplicaite & Mear \\
\hline l. & 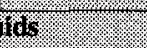 & (3.). & ITiniress & minifess & minitess \\
\hline S95T000978 & Riser 7 & Grab sample & 1.39 & 1.39 & 1.39 \\
\hline
\end{tabular}

\section{B2.3.3 pH}

The $\mathrm{pH}$ of grab sample U-108-1 was measured directly by glass electrode according to procedure LA-212-102, Rev. C-5. As shown in Table B2-9, the liquid was quite alkaline.

Table B2-9. Tank 241-U-108 Grab Sample Analytical Results: pH Measurement.

\begin{tabular}{|c|c|c|c|c|c|}
\hline 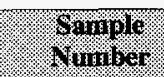 & $\begin{array}{l}\text { Gomplo } \\
\text { I Gentinan }\end{array}$ & $\begin{array}{l}\text { Samples } \\
\text { Portion. }\end{array}$ & Resail. & Buplicate & Mean \\
\hline l. & & & unitloss & anitless & initiess \\
\hline S95T000978 & Riser 7 & Grab sample & 13.73 & 13.70 & 13.72 \\
\hline
\end{tabular}




\section{B2.4 INORGANIC ANALYSES}

\section{B2.4.1 Total Organic Carbon}

Analyses for TOC were performed by direct persulfate oxidation on all 1996 core subsamples per procedure LA-342-100, Rev. D-0 and by furnace oxidation per procedure LA-344-105, Rev. B-2 on the 1995 grab sample. The TOC results from all cores are shown in Tables B2-10 and B2-11.

The lower half of segment 2 from core 146 had the lowest TOC (wet weight) concentration at $1,745 \mu \mathrm{g} / \mathrm{g}$. The highest concentration was $11,200 \mu \mathrm{g} / \mathrm{g}$ found in segment 9 of the same core. Even after conversion to a dry-weight basis, these values are below the programmatic dry weight notification limit of $30,000 \mu \mathrm{g} / \mathrm{g}$.

Table B2-10. Tank 241-U-108 Core Sample Analytical Results: Total Organic Carbon (Persulfate). ( 3 sheets)

\begin{tabular}{|c|c|c|c|c|c|c|}
\hline Sinipple & 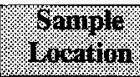 & $\begin{array}{l}\text { Simpole } \\
\text { Tortion }\end{array}$ & Resilt & Biplicate & Miplieare & Mean: \\
\hline \multicolumn{2}{|c|}{ S Srlat } & l. & .896 & $10 \mathrm{grg}$. & .696 & $\sqrt{1848}$ \\
\hline S96T002241 & 141: 1 & Lower $1 / 2$ & 6,000 & 6,560 & & 6,280 \\
\hline \$96T002242 & 141: 2 & Upper $1 / 2$ & $3, \overline{470}$ & 3,060 & & $3,265^{\mathrm{QC}: c}$ \\
\hline S96T002243 & $141: 3$ & Upper $1 / 2$ & 2,660 & 2,680 & & $2,670^{\mathrm{QC}: d}$ \\
\hline S96T002244 & 141: $3 A$ & Upper $1 / 2$ & 2,980 & 2,950 & & 2,965 \\
\hline S96T002245 & \multirow[t]{2}{*}{ 141: 4} & Upper $1 / 2$ & 2,750 & 4,430 & 4,600 & 3,927 \\
\hline S96T002246 & & Lower $1 / 2$ & 4,950 & 4,750 & & $4,850^{\mathrm{Q} C: \mathrm{c}}$ \\
\hline S96T002247 & \multirow[t]{2}{*}{ 141: 4A } & Upper $1 / 2$ & 4,440 & 4,560 & & 4,500 \\
\hline S96T002248 & & Lower $1 / 2$ & 4,350 & 4,570 & & 4,460 \\
\hline S96T002585 & \multirow[t]{2}{*}{$141: \overline{5}$} & Upper $1 / 2$ & 4,380 & 4,900 & & 4,640 \\
\hline S96T002586 & & Lower $1 / 2$ & 3,850 & 3,830 & & 3,840 \\
\hline S96T002587 & \multirow[t]{2}{*}{ 141: 6} & Upper $1 / 2$ & 3,380 & 3,280 & & 3,330 \\
\hline \$96T002588 & & Lower $1 / 2$ & 3,030 & 2,600 & & 2,815 \\
\hline S96T002589 & \multirow[t]{2}{*}{ 141: 7} & Upper $1 / 2$ & 6,550 & 6,080 & & 6,315 \\
\hline S96T002590 & & Lower $1 / 2$ & 5,260 & 5,270 & & 5,265 \\
\hline S96T002591 & \multirow[t]{2}{*}{ 141: 8} & Upper $1 / 2$ & 5,480 & 6,170 & & 5,825 \\
\hline S96T002592 & & Lower $1 / 2$ & 5,650 & 3,660 & 4,380 & 4,563 \\
\hline S96T002593 & 141: 9 & Upper $1 / 2$ & 2,290 & 2,630 & & 2,460 \\
\hline S96T002893 & 145: 2 & Upper $1 / 2$ & 2,610 & 2,760 & & 2,685 \\
\hline
\end{tabular}


Table B2-10. Tank 241-U-108 Core Sample Analytical Results: Total Organic Carbon (Persulfate). (3 sheets)

\begin{tabular}{|c|c|c|c|c|c|c|}
\hline $\begin{array}{l}\text { Somple. } \\
\text { Yimber. }\end{array}$ & $\begin{array}{l}\text { Samiries } \\
\text { locition. }\end{array}$ & $\begin{array}{l}\text { Sample: } \\
\text { rortion }\end{array}$ & Resuli: & Gipllonte & Mriplicate & Mesn \\
\hline \multicolumn{2}{|c|}{ 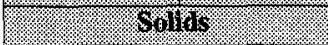 } & & 1988 & 1.189 & $108 \%$ & 3.4818 \\
\hline S96T002879 & \multirow[t]{2}{*}{$145: 3$} & Upper $1 / 2$ & 3,190 & 3,170 & & 3,180 \\
\hline S96T002880 & & Lower $1 / 2$ & $3, \overline{460}$ & 3,060 & & 3,260 \\
\hline S96T002881 & \multirow[t]{2}{*}{$145: 4$} & Upper $1 / 2$ & 2,920 & 2,950 & & 2,935 \\
\hline S96T002882 & & Lower $1 / 2$ & 3,870 & 3,860 & & 3,865 \\
\hline S96T002883 & \multirow[t]{2}{*}{$145: 5$} & Upper $1 / 2$ & 3,720 & 3,700 & & 3,710 \\
\hline S96T002884 & & Lower $1 / 2$ & 5,580 & 5,530 & & 5,555 \\
\hline S96T002885 & \multirow[t]{2}{*}{ 145: 6} & Upper $1 / 2$ & 4,850 & $4, \overline{920}$ & & 4,885 \\
\hline S96T002886 & & Lower $1 / 2$ & 3,980 & 4,230 & & $\overline{4,105}$ \\
\hline S96T002887 & \multirow[t]{2}{*}{$145: 7$} & Upper $1 / 2$ & 4,500 & 4,710 & & 4,605 \\
\hline S96T002888 & & Lower $1 / 2$ & 5,240 & 5,360 & & 5,300 \\
\hline S96T002889 & \multirow[t]{2}{*}{$145: 8$} & Upper $1 / 2$ & 2,900 & 2,880 & & 2,890 \\
\hline S96T002890 & & Lower $1 / 2$ & 4,530 & 4,650 & & 4,590 \\
\hline S96T002891 & \multirow[t]{2}{*}{$145: 9$} & Upper $1 / 2$ & 5,350 & 5,090 & & 5,220 \\
\hline S96T002892 & & Lower $1 / 2$ & 2,730 & 2,860 & & 2,795 \\
\hline S96T003121 & 146: 1 & Upper $1 / 2$ & 3,920 & 4,000 & & 3,960 \\
\hline S96T003123 & \multirow[t]{2}{*}{ 146: 2} & Upper $1 / 2$ & 2,820 & 2,790 & & 2,805 \\
\hline S96T003124 & & Lower $1 / 2$ & 1,780 & 1,710 & & 1,745 \\
\hline S96T003125 & \multirow[t]{2}{*}{$146: 3$} & Upper $1 / 2$ & 2,960 & 2,740 & 2,600 & 2,767 \\
\hline S96T003126 & & Lower $1 / 2$ & 2,250 & 2,630 & 2,140 & 2,340 \\
\hline S96T002959 & 146: $3 A$ & Upper $1 / 2$ & 2,620 & 2,730 & 3,930 & 3,093 \\
\hline S96T002960 & \multirow[t]{2}{*}{ 146: 4} & Upper $1 / 2$ & 2,640 & 2,600 & & 2,620 \\
\hline S96T002961 & & Lower $1 / 2$ & 4,270 & 4,150 & & 4,210 \\
\hline S96T002962 & \multirow[t]{2}{*}{ 146: 5} & Upper $1 / 2$ & 4,650 & 5,080 & & 4,865 \\
\hline S96T002963 & & Lower $1 / 2$ & 4,330 & 3,850 & & 4,090 \\
\hline S96T002964 & \multirow[t]{2}{*}{ 146: 6} & Upper $1 / 2$ & 4,180 & 4,220 & & 4,200 \\
\hline S96T002965 & & Lower $1 / 2$ & 4,430 & 4,440 & & 4,435 \\
\hline S96T002966 & \multirow[t]{2}{*}{ 146: 7} & Upper $1 / 2$ & 4,960 & 5,750 & & 5,355 \\
\hline S96T002967 & & Lower $1 / 2$ & 7,430 & 6,310 & & $6,870^{\overline{Q C: c}}$ \\
\hline S96T003127 & \multirow[t]{2}{*}{ 146: 8} & Upper $1 / 2$ & 6,010 & 5,980 & 5,200 & 5,730 \\
\hline S96T003128 & & Lower $1 / 2$ & 3,350 & 3,230 & & 3,290 \\
\hline S96T004178 & \multirow[t]{2}{*}{ 146: 9} & Lower $1 / 2$ & 5,540 & 5,180 & & $5,360^{\mathrm{QC}: \mathrm{d}}$ \\
\hline S96T003129 & & Upper $1 / 2$ & 11,100 & 11,200 & & 11,150 \\
\hline
\end{tabular}


Table B2-10. Tank 241-U-108 Core Sample Analytical Results: Total Organic Carbon (Persulfate). (3 sheets)

\begin{tabular}{|c|c|c|c|c|c|c|}
\hline Whingle & oromple & Samplo & Irsulle & Oiviluate & Hiplicate & Mean \\
\hline \multicolumn{2}{|c|}{. } & & 1098. & $64 \mathrm{~g}=$ & 1084 & $14 \mathrm{~g}$ \\
\hline S96T003449 & Core 141 & $\begin{array}{l}\text { Solid } \\
\text { composite }\end{array}$ & 5,100 & 5,080 & & 5,090 \\
\hline S96T003659 & Core 145 & $\begin{array}{l}\text { Solid } \\
\text { composite }\end{array}$ & 4,520 & 4,770 & & 4,645 \\
\hline S96T004198 & Core 146 & $\begin{array}{l}\text { Solid } \\
\text { composite }\end{array}$ & 3,510 & 3,850 & & 3,680 \\
\hline \multicolumn{2}{|c|}{ Glinins } & & $4+213$ & $198 \%$ & 14111 & $149 \%$ \\
\hline S96T002282 & 141: 1 & $\begin{array}{l}\text { Drainable } \\
\text { liquid }\end{array}$ & 7,030 & 7,150 & & $7,090^{\mathrm{eC:}: \mathrm{f}}$ \\
\hline S96T002942 & 145: 1 & $\begin{array}{l}\text { Drainable } \\
\text { liquid }\end{array}$ & 6,990 & $7, \overline{040}$ & & 7,015 \\
\hline S96T003163 & 146: 1 & $\begin{array}{l}\text { drainable } \\
\text { liquid }\end{array}$ & 7,240 & 6,970 & & $7,105^{\mathrm{QC:f}}$ \\
\hline
\end{tabular}

Table B2-11. Tank 241-U-108 Grab Sample Analytical Results: Total Organic Carbon (Furnace Oxidation).

\begin{tabular}{|c|c|c|c|c|c|}
\hline Squple & Whan & Gormple & Scsur & Bupleate & rosin \\
\hline \multicolumn{2}{|c|}{ (3.1. } & & 441 & Hafril & 4. \\
\hline S95T000978 & Riser 7 & Grab sample & 7,490 & 7,390 & 7,440 \\
\hline
\end{tabular}

\section{B2.4.2 Total Inorganic Carbon}

Analyses for TIC were performed directly on all solid and drainable liquid samples from all cores using procedure LA-342-100, Rev. D-0, and on the 1995 grab sample using procedure LA-622-102, Rev. B-2. Carbonate was determined by the TIC method instead of IC as specified in the core SAP. Most of the TIC levels from all solid and drainable liquid samples were consistent. Results (Tables B2-12 and B2-13) varied from a high of $16,400 \mu \mathrm{g} / \mathrm{g}$ in the lower half of segment 7 from core 146, to $844.5 \mu \mathrm{g} / \mathrm{g}$ in the upper half of segment 9 from core 141 . 
Table B2-12. Tank 241-U-108 Core Sample Analytical Results: Total Inorganic Carbon. (2 sheets)

\begin{tabular}{|c|c|c|c|c|c|c|}
\hline Whinos & 6010\%) & Waraplo & Yexilit & Suplicate & mivingre & Wean \\
\hline \multicolumn{3}{|c|}{ (2. } & 1898 & 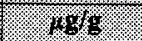 & $168 \mathrm{~s}$. & 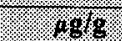 \\
\hline S96T002241 & 141: 1 & Lower $1 / 2$ & 6,880 & 6,920 & & 6,900 \\
\hline S96T002242 & 141: 2 & Upper $1 / 2$ & 6,360 & 5,700 & & 6,030 \\
\hline S96T002243 & 141: 3 & Upper $1 / 2$ & 5,230 & 5,410 & & 5,320 \\
\hline S96T002244 & 141: $3 \mathrm{~A}$ & Upper $1 / 2$ & 3,420 & 3,290 & & 3,355 \\
\hline S96T002245 & \multirow[t]{2}{*}{ 141: 4} & Upper $1 / 2$ & 6,880 & 6,930 & 6,910 & 6,907 \\
\hline S96T002246 & & Lower $1 / 2$ & 7,660 & 7,860 & & 7,760 \\
\hline S96T002247 & \multirow[t]{2}{*}{ 141: $4 \mathrm{~A}$} & Upper $1 / 2$ & 6,720 & 7,080 & & 6,900 \\
\hline S96T002248 & & Lower $1 / 2$ & 7,190 & 7,290 & & 7,240 \\
\hline S96T002585 & \multirow[t]{2}{*}{ 141: 5} & Upper $1 / 2$ & 7,270 & 7,980 & & 7,625 \\
\hline S96T002586 & & Lower $1 / 2$ & 6,790 & $6,7 \overline{40}$ & & 6,765 \\
\hline S96T002587 & \multirow[t]{2}{*}{$141: 6$} & Upper $1 / 2$ & 6,870 & 6,710 & & 6,790 \\
\hline S96T002588 & & Lower $1 / 2$ & 6,570 & 6,390 & & 6,480 \\
\hline S96T002589 & \multirow[t]{2}{*}{$141: 7$} & Upper $1 / 2$ & 13,100 & 14,100 & & 13,600 \\
\hline S96T002590 & & Lower $1 / 2$ & 12,400 & 13,100 & & 12,750 \\
\hline S96T002591 & \multirow[t]{2}{*}{ 141: 8} & Upper 1/2 & 13,700 & 11,800 & & 12,750 \\
\hline \$96T002592 & & Lower $1 / 2$ & 11,800 & 11,900 & 11,500 & 11,733 \\
\hline S96T002593 & 141: 9 & Upper $1 / 2$ & 892 & 797 & & 844.5 \\
\hline S96T002893 & 145: 2 & Upper $1 / 2$ & 3,040 & 3,360 & & 3,200 \\
\hline S96T002879 & \multirow[t]{2}{*}{ 145: 3} & Upper $1 / 2$ & 4,620 & 4,800 & & 4,710 \\
\hline S96T002880 & & Lower $1 / 2$ & 4,810 & 4,630 & & 4,720 \\
\hline S96T002881 & \multirow[t]{2}{*}{$145: 4$} & Upper $1 / 2$ & 4,850 & 5,020 & & 4,935 \\
\hline S96T002882 & & Lower $1 / 2$ & 5,680 & 5,740 & & 5,710 \\
\hline S96T002883 & \multirow[t]{2}{*}{$145: 5$} & Upper $1 / 2$ & 5,940 & 6,080 & & 6,010 \\
\hline S96T002884 & & Lower $1 / 2$ & 11,000 & 10,800 & & 10,900 \\
\hline S96T002885 & \multirow[t]{2}{*}{ 145: 6} & Upper $1 / 2$ & 11,300 & 11,200 & & 11,250 \\
\hline S96T002886 & & Lower $1 / 2$ & 10,200 & 10,300 & & 10,250 \\
\hline S96T002887 & \multirow[t]{2}{*}{ 145: 7} & Upper $1 / 2$ & 12,300 & 10,500 & & 11,400 \\
\hline S96T002888 & & Lower $1 / 2$ & 13,100 & 12,900 & & 13,000 \\
\hline S96T002889 & \multirow[t]{2}{*}{ 145: 8} & Upper $1 / 2$ & 8,350 & 7,930 & & 8,140 \\
\hline S96T002890 & & Lower $1 / 2$ & 13,000 & 13,200 & & 13,100 \\
\hline S96T002891 & \multirow[t]{2}{*}{ 145: 9} & Upper $1 / 2$ & 11,700 & 13,300 & & 12,500 \\
\hline S96T002892 & & Lower $1 / 2$ & 1,320 & 1,430 & & 1,375 \\
\hline
\end{tabular}


Table B2-12. Tank 241-U-108 Core Sample Analytical Results: Total Inorganic Carbon. (2 sheets)

\begin{tabular}{|c|c|c|c|c|c|c|}
\hline Shanger & Gamis & 3rimol & hesali: & Diplicate. & Wriplitate & 14641 \\
\hline \multicolumn{3}{|c|}{ 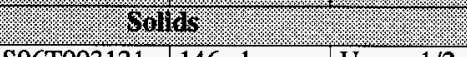 } & $1+8 / 8$ & $1+88$ & 1.86 & 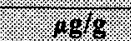 \\
\hline S96T003121 & 146: 1 & Upper $1 / 2$ & 10,300 & 9,070 & & 9,685 \\
\hline S96T003123 & \multirow[t]{2}{*}{$146: 2$} & Upper $1 / 2$ & 6,440 & 5,590 & & $6, \overline{015}$ \\
\hline S96T003124 & & Lower $1 / 2$ & 1,400 & 1,400 & & 1,400 \\
\hline S96T003125 & \multirow[t]{2}{*}{ 146: 3} & Upper $1 / 2$ & 7,190 & 7,680 & 7,620 & $7, \overline{497}$ \\
\hline S96T003126 & & Lower $1 / 2$ & 2,080 & 2,440 & & 2,260 \\
\hline S96T002959 & 146: $3 \mathrm{~A}$ & Upper $1 / 2$ & 4,210 & 5,760 & 3,910 & 4,627 \\
\hline S96T002960 & \multirow[t]{2}{*}{ 146: 4} & Upper $1 / 2$ & 4,060 & 4,020 & & 4,040 \\
\hline S96T002961 & & Lower $1 / 2$ & 9,470 & 9,410 & & 9,440 \\
\hline S96T002962 & \multirow[t]{2}{*}{$146: 5$} & Upper $1 / 2$ & 11,500 & 11,900 & & 11,700 \\
\hline S96T002963 & & Lower $1 / 2$ & 9,750 & 8,830 & & 9,290 \\
\hline S96T002964 & \multirow[t]{2}{*}{ 146: 6} & Upper $1 / 2$ & 10,500 & 10,700 & & 10,600 \\
\hline S96T002965 & & Lower $1 / 2$ & 11,700 & 11,600 & & 11,650 \\
\hline S96T002966 & \multirow[t]{2}{*}{ 146: 7} & Upper $1 / 2$ & 12,200 & 12,000 & & 12,100 \\
\hline S96T002967 & & Lower $1 / 2$ & 16,200 & 16,600 & & 16,400 \\
\hline S96T003127 & \multirow[t]{2}{*}{ 146: 8} & Upper $1 / 2$ & 10,500 & 12,800 & 13,800 & 12,370 \\
\hline S96T003128 & & Lower $1 / 2$ & 7,680 & 8,060 & & 7,870 \\
\hline S96T004178 & \multirow[t]{2}{*}{ 146: 9} & Lower $1 / 2$ & 1,220 & 1,220 & & 1,220 \\
\hline \$96T003129 & & Upper $1 / 2$ & 14,600 & 15,100 & & 14,850 \\
\hline S96T003449 & Core 141 & $\begin{array}{l}\text { Solid } \\
\text { composite }\end{array}$ & 7,590 & 7,800 & & 7,695 \\
\hline S96T003659 & Core 145 & $\begin{array}{l}\text { Solid } \\
\text { composite }\end{array}$ & 10,000 & 10,000 & & 10,000 \\
\hline S96T004198 & Core 146 & $\begin{array}{l}\text { Solid } \\
\text { composite }\end{array}$ & 7,080 & 8,100 & & 7,590 \\
\hline \multicolumn{2}{|c|}{ Wouros } & & 10/111 & 1.901171 & / glin! & $48 \mathrm{ml}$ \\
\hline S96T002282 & $141: 1$ & $\begin{array}{l}\text { Drainable } \\
\text { liquid }\end{array}$ & 4,220 & 4,750 & & 4,485 \\
\hline S96T002942 & 145: 1 & $\begin{array}{l}\text { Drainable } \\
\text { liquid }\end{array}$ & 4,070 & 4,490 & & 4,280 \\
\hline S96T003163 & 146: 1 & $\begin{array}{l}\text { Drainable } \\
\text { liquid }\end{array}$ & 4,340 & 4,510 & & 4,425 \\
\hline
\end{tabular}


Table B2-13. Tank 241-U-108 Grab Sample Analytical Results: Total Inorganic Carbon.

\begin{tabular}{|c|c|c|c|c|c|}
\hline Homolo & 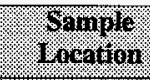 & Pormol & Rerul & Buploate & Morn \\
\hline \multicolumn{2}{|c|}{ Mging } & & (x) 6 min & $4 \mathrm{~g}_{111}$ & 198111 \\
\hline S95T000978 & Riser 7 & Grab sample & 3,200 & 3,220 & 3,210 \\
\hline
\end{tabular}

\section{B2.4.3 Hydroxide}

Analysis for hydroxide was performed directly by titration on the 1995 grab sample U-108-1 per procedure LA-211-102, Rev. C-2. Results are shown in Table B2-14.

Table B2-14. Tank 241-U-108 Grab Sample Analytical Results: Hydroxide.

\begin{tabular}{|c|c|c|c|c|c|}
\hline 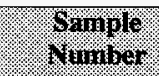 & Shoupor & gorision & Meswilt & Huplliste & Mron \\
\hline \multicolumn{3}{|c|}{ 1. } & $(48414$ & pgrol & \%\$11 \\
\hline S95T000978 & Riser 7 & Grab sample & 48,400 & 48,900 & 49,200 \\
\hline
\end{tabular}

\section{B2.4.4 Inductively Coupled Plasma}

Samples were analyzed directly following acid dilution or were prepared by either fusion, acid, or water digests. The ICP analyses on core samples were performed per procedures LA-505-161, Rev. D-3 (per the data package), or LA-505-151, Rev. D-3, depending on the ICP instrument used. The ICP analyses on the 1995 grab sample were performed per procedure LA-505-161, Rev. A-1. Although a full suite of analytes were reported on the acid- and water-digested 1996 core samples, the analytes of interest were $\mathrm{Al}, \mathrm{Bi}, \mathrm{Ca}, \mathrm{Cr}, \mathrm{Fe}$, $\mathrm{Li}, \mathrm{Mn}, \mathrm{Na}, \mathrm{Ni}, \mathrm{P}, \mathrm{Si}$, and $\mathrm{U}$. The ICP analytes $\mathrm{Al}, \mathrm{Fe}, \mathrm{Cr}, \mathrm{Bi}, \mathrm{Na}, \mathrm{Ni}, \mathrm{Si}$, and $\mathrm{U}$ were required for historical waste modeling per the historical DQO. The metals $\mathrm{Al}, \mathrm{Fe}$, and $\mathrm{Na}$ were required by the compatibility $\mathrm{DQO}$. Lithium was required to evaluate contamination by hydrostatic head fluid and/or wash water used during sampling. The analytes $\mathrm{Al}, \mathrm{Bi}, \mathrm{Ca}$, $\mathrm{Fe}, \mathrm{P}, \mathrm{Na}$, and $\mathrm{Mn}$ are required by the organic $\mathrm{DQO}$ as secondary analytes if the TOC exceeds $30,000 \mu \mathrm{g} / \mathrm{g}$. Because no TOC values exceeded that value, Mn was not a required analyte. The potassium and zirconium results for the ICP fusion analyses should be disregarded, because the samples were prepared in a zirconium crucible by fusion using potassium hydroxide. For the core samples, additional ICP data were collected on an opportunistic basis (Kristofzski 1996) and are reported here. Because these data were not identified in any DQO document, there were no programmatic QC requirements with respect to these data, and the QC information associated with them were not evaluated for this report. 
The ICP results are shown in Tables B2-16 through B2-37 for the 1996 core sample solids and drainable liquids, and on the 1995 liquid grab sample. Many ICP analytes were not detected at all for a given sample (digestate). These analytes are listed in Table B2-15 along with the respective digest and the highest detection limit. The solid core composite samples were prepared by fusion, water and acid digestion prior to analysis, the solid core samples were prepared by fusion digestion, and liquids were analyzed directly following acid dilution.

Table B2-15. Non-Detected ICP Analytes for Tank 241-U-108 Core Samples. (2 sheets)

\begin{tabular}{|c|c|c|}
\hline (. & $8486 s t$ & 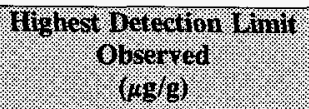 \\
\hline Arsenic & acid & 98.9 \\
\hline Arsenic & water & 103 \\
\hline Arsenic & none & 40.1 \\
\hline Barium & acid & 49.5 \\
\hline Barium & water & 51.3 \\
\hline Barium & none & 20.1 \\
\hline Beryllium & acid & 4.95 \\
\hline Beryllium & water & 5.13 \\
\hline Beryllium & none & 2 \\
\hline Bismuth & acid & 98.9 \\
\hline Bismuth & fusion & 2,270 \\
\hline Bismuth & water & 103 \\
\hline Bismuth & none & 40.1 \\
\hline Calcium & fusion & 2,270 \\
\hline Cerium & acid & 98.9 \\
\hline Cerium & water & 103 \\
\hline Cerium & none & 40.1 \\
\hline Cobalt & acid & 19.8 \\
\hline Cobalt & water & 20.5 \\
\hline Cobalt & none & 8.02 \\
\hline Lanthanum & acid & 49.5 \\
\hline Lanthanum & water & 51.3 \\
\hline Lanthanum & none & 20.1 \\
\hline Lead & acid & 98.9 \\
\hline Lead & water & 103 \\
\hline Lead & none & 40.1 \\
\hline Lithium & acid & 9.89 \\
\hline
\end{tabular}


Table B2-15. Non-Detected ICP Analytes for Tank 241-U-108 Core Samples. (2 sheets)

\begin{tabular}{|c|c|c|}
\hline 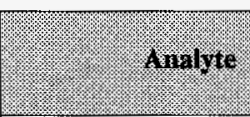 & 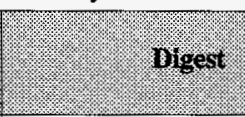 & 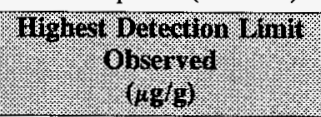 \\
\hline Lithium & fusion & 227 \\
\hline Lithium & water & 10.3 \\
\hline Magnesium & acid & 98.9 \\
\hline Magnesium & water & 103 \\
\hline Magnesium & none & 40.1 \\
\hline Neodymium & acid & 98.9 \\
\hline Neodymium & water & $\overline{103}$ \\
\hline Neodymium & none & 40.1 \\
\hline Samarium & acid & 98.9 \\
\hline Samarium & water & 103 \\
\hline Samarium & none & 40.1 \\
\hline Selenium & acid & 98.9 \\
\hline Selenium & water & 103 \\
\hline Selenium & none & 40.1 \\
\hline Strontium & acid & 9.89 \\
\hline Strontium & water & 10.3 \\
\hline Strontium & none & 4.01 \\
\hline Thallium & acid & 198 \\
\hline Thallium & water & 205 \\
\hline Thallium & none & 80.2 \\
\hline Titanium & acid & 9.89 \\
\hline Titanium & water & 10.3 \\
\hline Tantalum & none & 4.01 \\
\hline Uranium & acid & 495 \\
\hline Uranium & fusion & 11,400 \\
\hline Uranium & water & 513 \\
\hline Uranium & none & 200 \\
\hline Vanadium & acid & 49.5 \\
\hline Vanadium & water & 51.3 \\
\hline Vanadium & none & 20.1 \\
\hline Zirconium & acid & 9.89 \\
\hline Zirconium & water & 10.3 \\
\hline Zirconium & none & 4.01 \\
\hline
\end{tabular}


Table B2-16. Tank 241-U-108 Core Sample Analytical Results: Aluminum (ICP). ( 3 sheets)

\begin{tabular}{|c|c|c|c|c|c|}
\hline $\begin{array}{l}\text { Sarmple: } \\
\text { Tumber. }\end{array}$ & $\begin{array}{l}\text { Sampie } \\
\text { Locition }\end{array}$ & $\begin{array}{l}\text { Sample } \\
\text { Rortion }\end{array}$ & Tesuli & Buplicate & Mean \\
\hline \multicolumn{2}{|c|}{ Sollat aedoligest. } & & 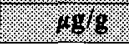 & 1.48 & $6.78 \mathrm{~s} / \mathrm{s}$ \\
\hline S96T003451 & Core 141 & $\begin{array}{l}\text { Solid } \\
\text { composite }\end{array}$ & 26,400 & 25,500 & $25,950^{\mathrm{QC}: \mathrm{b}}$ \\
\hline S96T003457 & Core 145 & $\begin{array}{l}\text { Solid } \\
\text { composite }\end{array}$ & 15,000 & 14,500 & $14,750^{\mathrm{QC}: b}$ \\
\hline S96T004200 & Core 146 & $\begin{array}{l}\text { Solid } \\
\text { composite }\end{array}$ & 12,900 & 16,200 & $14,550^{\mathrm{QC}: c, \mathrm{e}}$ \\
\hline \multicolumn{2}{|c|}{ Sollids. Wusion } & (3. & $.48 \%$ & $1.48 \%$ & $.98 \%$ \\
\hline S96T002257 & 141: 1 & Lower $1 / 2$ & 23,700 & 22,600 & 23,150 \\
\hline S96T002258 & 141: 2 & Upper $1 / 2$ & 11,900 & $12, \overline{500}$ & 12,200 \\
\hline S96T002259 & $141: 3$ & Upper $1 / 2$ & 12,200 & 11,900 & 12,050 \\
\hline S96T002260 & $141: 3 A$ & Upper $1 / 2$ & 11,200 & 10,600 & 10,900 \\
\hline S96T002261 & $141: 4$ & Upper $1 / 2$ & 15,400 & 16,500 & 15,950 \\
\hline S96T002262 & & Lower $1 / 2$ & 18,800 & 18,000 & 18,400 \\
\hline S96T002263 & 141: $4 \mathrm{~A}$ & Upper $1 / 2$ & 17,100 & 17,300 & 17,200 \\
\hline S96T002264 & & Lower $1 / 2$ & 15,600 & 17,500 & $16,5 \overline{50^{\mathrm{QC}: \mathrm{e}}}$ \\
\hline S96T002608 & $141: 5$ & Upper $1 / 2$ & 16,800 & 16,400 & 16,600 \\
\hline S96T002609 & & Lower $1 / 2$ & 13,500 & 14,600 & 14,050 \\
\hline S96T002610 & $141: 6$ & Upper $1 / 2$ & 14,000 & 13,300 & 13,650 \\
\hline S96T002611 & & Lower $1 / 2$ & 13,300 & 13,200 & 13,250 \\
\hline S96T002612 & 141: 7 & Upper $1 / 2$ & 21,400 & 20,900 & 21,150 \\
\hline S96T002613 & & Lower $1 / 2$ & 17,900 & 16,500 & 17,200 \\
\hline S96T002614 & 141: 8 & Upper $1 / 2$ & 20,300 & 20,900 & 20,600 \\
\hline S96T002615 & & Lower $1 / 2$ & 15,000 & 15,700 & 15,350 \\
\hline S96T002616 & 141: 9 & Upper $1 / 2$ & 83,500 & 74,400 & $78,950^{\mathrm{QC}: \mathrm{e}}$ \\
\hline S96T002909 & $145: 2$ & Upper $1 / 2$ & 9,690 & 10,500 & 10,095 \\
\hline S96T002910 & $145: 3$ & Upper $1 / 2$ & 12,500 & 12,400 & $12, \overline{450}$ \\
\hline S96T002911 & & Lower $1 / 2$ & 12,600 & 12,100 & 12,350 \\
\hline S96T002912 & $145: 4$ & Upper $1 / 2$ & 11,500 & 11,200 & 11,350 \\
\hline S96T002913 & & Lower $1 / 2$ & 15,100 & $14, \overline{200}$ & 14,650 \\
\hline S96T002914 & $145: 5$ & Upper $1 / 2$ & 13,600 & 14,400 & 14,000 \\
\hline S96T002915 & & Lower $1 / 2$ & 21,500 & 20,800 & 21,150 \\
\hline S96T002916 & $145: 6$ & Upper $1 / 2$ & 16,100 & 15,000 & 15,550 \\
\hline S96T002917 & & Lower $1 / 2$ & 13,900 & 14,100 & 14,000 \\
\hline
\end{tabular}


Table B2-16. Tank 241-U-108 Core Sample Analytical Results: Aluminum (ICP). (3 sheets)

\begin{tabular}{|c|c|c|c|c|c|}
\hline 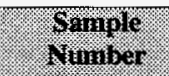 & $\begin{array}{l}\text { Samile } \\
\text { iccition }\end{array}$ & $\begin{array}{l}\text { Sample: } \\
\text { iortion }\end{array}$ & . Nesint & Diplicate & Mean \\
\hline \multicolumn{2}{|c|}{ 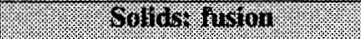 } & (.. & 1.818 & 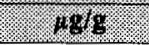 & 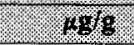 \\
\hline S96T002918 & $145: 7$ & Upper $1 / 2$ & 15,200 & 14,700 & 14,950 \\
\hline S96T002919 & & Lower $1 / 2$ & 17,400 & 17,200 & 17,300 \\
\hline S96T002920 & $145: 8$ & Upper $1 / 2$ & 10,300 & $10, \overline{400}$ & 10,350 \\
\hline S96T002921 & & Lower $\overline{1 / 2}$ & 12,700 & 13,300 & 13,000 \\
\hline S96T002922 & $145: 9$ & Upper $1 / 2$ & 13,500 & 13,400 & 13,450 \\
\hline S96T002923 & & Lower $1 / 2$ & 7,530 & 8,030 & 7,780 \\
\hline S96T003143 & 146: 1 & Upper $1 / 2$ & 14,200 & 15,400 & $14, \overline{800}$ \\
\hline S96T003144 & $146: 2$ & Upper $1 / 2$ & 10,100 & 10,700 & 10,400 \\
\hline S96T003145 & & Lower $1 / 2$ & 6,900 & 7,320 & 7,110 \\
\hline S96T003146 & $146: 3$ & Upper $1 / 2$ & $10, \overline{000}$ & 9,130 & 9,565 \\
\hline S96T003147 & & Lower $1 / 2$ & 8,410 & 8,210 & 8,310 \\
\hline S96T003001 & 146: $3 A$ & Upper $1 / 2$ & $9, \overline{940}$ & 8,660 & 9,000 \\
\hline S96T003002 & 146: 4 & Upper $1 / 2$ & 9,990 & 10,100 & 10,045 \\
\hline S96T003003 & & Lower $1 / 2$ & 15,900 & 15,400 & 15,650 \\
\hline S96T003004 & $146: 5$ & Upper $1 / 2$ & 19,300 & 19,600 & 19,450 \\
\hline S96T003005 & & Lower $1 / 2$ & 14,200 & 13,900 & 14,050 \\
\hline S96T003006 & 146: 6 & Upper $1 / 2$ & 14,700 & 14,100 & 14,400 \\
\hline S96T003007 & & Lower $1 / 2$ & 16,200 & 16,200 & 16,200 \\
\hline S96T003008 & 146: 7 & Upper $\overline{1 / 2}$ & 17,000 & 18,200 & 17,600 \\
\hline S96T003009 & & Lower $1 / 2$ & 20,800 & 20,900 & 20,850 \\
\hline S96T003148 & 146: 8 & Upper $1 / 2$ & 21,300 & 20,500 & 20,900 \\
\hline S96T003149 & & Lower $1 / 2$ & 19,700 & 20,600 & 20,150 \\
\hline S96T004180 & $146: 9$ & Lower $1 / 2$ & 9,470 & 9,320 & 9,395 \\
\hline S96T003150 & & Upper $1 / 2$ & 16,200 & $16, \overline{400}$ & 16,300 \\
\hline S96T003450 & Core 141 & $\begin{array}{l}\text { Solid } \\
\text { composite }\end{array}$ & 27,300 & 23,000 & $25,150^{\mathrm{QC}: \mathrm{e}}$ \\
\hline S96T003660 & Core 145 & $\begin{array}{l}\text { Solid } \\
\text { composite }\end{array}$ & 19,700 & 12,300 & $16,000^{\mathrm{QC}: \mathrm{e}}$ \\
\hline S96T004199 & Core 146 & $\begin{array}{l}\text { Solid } \\
\text { composite }\end{array}$ & 15,200 & 15,400 & 15,300 \\
\hline
\end{tabular}


Table B2-16. Tank 241-U-108 Core Sample Analytical Results: Aluminum (ICP). ( 3 sheets)

\begin{tabular}{|c|c|c|c|c|c|}
\hline $\begin{array}{l}\text { Sarmple } \\
\text { Runber. }\end{array}$ & $\begin{array}{l}\text { Sample } \\
\text { Isocition }\end{array}$ & $\begin{array}{l}\text { Shinple } \\
\text { Portion }\end{array}$ & Resint & Buplicate: & Miean \\
\hline \multicolumn{2}{|c|}{ Sollith: rater digest } & 2.: & 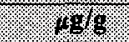 & 1948. & $10 \mathrm{~g} / \mathrm{B}$ \\
\hline S96T003453 & Core 141 & $\begin{array}{l}\text { Solid } \\
\text { composite }\end{array}$ & 21,700 & 21,300 & 21,500 \\
\hline S96T003662 & Core 145 & $\begin{array}{l}\text { Solid } \\
\text { composite }\end{array}$ & 14,500 & 13,900 & 14,200 \\
\hline S96T004202 & Core 146 & $\begin{array}{l}\text { Solid } \\
\text { composite }\end{array}$ & 13,800 & 13,800 & 13,800 \\
\hline \multicolumn{2}{|c|}{ IItuats. } & 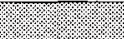 & $104 \mathrm{nin}$ & Womi: & 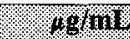 \\
\hline S96T002282 & $141: 1$ & $\begin{array}{l}\text { Drainable } \\
\text { liquid }\end{array}$ & 29,900 & 30,200 & 30,050 \\
\hline S96T002942 & 145: 1 & $\begin{array}{l}\text { Drainable } \\
\text { liquid }\end{array}$ & 31,100 & 31,100 & 31,100 \\
\hline S96T003163 & 146: 1 & $\begin{array}{l}\text { Drainable } \\
\text { liquid }\end{array}$ & 30,900 & 29,600 & 30,250 \\
\hline
\end{tabular}

Table B2-17. Tank 241-U-108 Grab Sample Analytical Results: Aluminum (ICP).

\begin{tabular}{|c|c|c|c|c|c|}
\hline Suningle & $\begin{array}{l}\text { Gimple } \\
\text { y. ocatron }\end{array}$ & $\begin{array}{l}\text { Squiplis } \\
\text { Pontion. }\end{array}$ & Res:uit & Supilcate & Mran \\
\hline \multicolumn{2}{|c|}{ 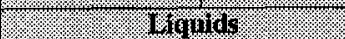 } & & Hotmis & $1.9 \mathrm{gm}$ & $108 \%$ \\
\hline S95T000978 & Riser 7 & Grab sample & 42,900 & 37,440 & 40,170 \\
\hline
\end{tabular}


Table B2-18. Tank 241-U-108 Core Sample Analytical Results: Antimony (ICP).

\begin{tabular}{|c|c|c|c|c|c|}
\hline $\begin{array}{l}\text { sompler } \\
\text { Number. }\end{array}$ & $\begin{array}{l}\text { Sormple } \\
\text { Ureation }\end{array}$ & $\begin{array}{l}\text { Saraple: } \\
\text { Portion. }\end{array}$ & Resul & Duplicate & Miean. \\
\hline \multicolumn{3}{|c|}{ Shios acid digest } & .408 & 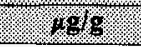 & $.49 / \mathrm{g}$ \\
\hline S96T003451 & Core 141 & $\begin{array}{l}\text { Solid } \\
\text { composite }\end{array}$ & 77.1 & $<57.8$ & $<67.45^{\mathrm{QC:}}$ \\
\hline S96T003457 & Core 145 & $\begin{array}{l}\text { Solid } \\
\text { composite }\end{array}$ & $<59.4$ & $<58$ & $<\overline{58.7}$ \\
\hline S96T004200 & Core 146 & $\begin{array}{l}\text { Solid } \\
\text { composite }\end{array}$ & $<28.9$ & $<31.5$ & $<30.2^{\mathrm{QC}: \mathrm{a}}$ \\
\hline \multicolumn{2}{|c|}{ Sollids yater diget } & 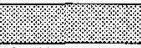 & 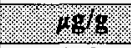 & 1818 & .9898 \\
\hline S96T003453 & Core 141 & $\begin{array}{l}\text { Solid } \\
\text { composite }\end{array}$ & $<61.6$ & $<61.5$ & $<61.55$ \\
\hline S96T003662 & Core 145 & $\begin{array}{l}\text { Solid } \\
\text { composite }\end{array}$ & $<56.5$ & $<43.7$ & $<50.1^{\mathrm{QC}: \mathrm{e}}$ \\
\hline S96T004202 & Core 146 & $\begin{array}{l}\text { Solid } \\
\text { composite }\end{array}$ & $<37$ & $<33$ & $<35^{\mathrm{Qce} \mathrm{e}}$ \\
\hline \multicolumn{2}{|c|}{ Virginds } & 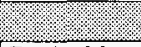 & $.1 \%$ & $. / 8 / \mathrm{mil}$ & 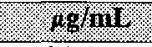 \\
\hline S96T002282 & 141: 1 & $\begin{array}{l}\text { Drainable } \\
\text { liquid }\end{array}$ & $<24.1$ & $<24.1$ & $<24.1$ \\
\hline S96T002942 & 145: 1 & $\begin{array}{l}\text { Drainable } \\
\text { liquid }\end{array}$ & $<24.1$ & $<24.1$ & $<24.1$ \\
\hline S96T003163 & 146: 1 & $\begin{array}{l}\text { Drainable } \\
\text { liquid }\end{array}$ & $<24.1$ & $<24.1$ & $<24.1$ \\
\hline
\end{tabular}


HNF-SD-WM-ER-639 Rev. 0

Table B2-19. Tank 241-U-108 Core Sample Analytical Results: Boron (ICP).

\begin{tabular}{|c|c|c|c|c|c|}
\hline Sample & Samiolis & $\begin{array}{l}\text { Gainglo } \\
\text { Prortion }\end{array}$ & Resillit & Buplicate & Mear \\
\hline \multicolumn{2}{|c|}{ Solids: acir digest } & (2) & 198: & . $\mu \mathrm{g} / \mathrm{g}$ & $198 \%$ \\
\hline S96T003451 & Core 141 & $\begin{array}{l}\text { Solid } \\
\text { composite }\end{array}$ & 106 & 117 & 111.5 \\
\hline S96T003457 & Core 145 & $\begin{array}{l}\text { Solid } \\
\text { composite }\end{array}$ & 71.2 & 64.5 & 67.85 \\
\hline S96T004200 & Core 146 & $\begin{array}{l}\text { Solid } \\
\text { composite }\end{array}$ & 57.6 & 66.2 & $61.9^{\mathrm{QC}: \mathrm{e}}$ \\
\hline \multicolumn{2}{|c|}{ Sollder vater digest } & (2) & 4.48 & 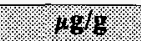 & 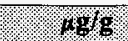 \\
\hline S96T003453 & Core 141 & $\begin{array}{l}\text { Solid } \\
\text { composite }\end{array}$ & 530 & 604 & $567^{\mathrm{QC:e}}$ \\
\hline S96T003662 & Core 145 & $\begin{array}{l}\text { Solid } \\
\text { composite }\end{array}$ & 538 & 454 & $496^{\mathrm{QC}: \mathrm{e}}$ \\
\hline S96T004202 & Core 146 & $\begin{array}{l}\text { Solid } \\
\text { composite }\end{array}$ & 589 & 431 & $510^{\mathrm{QC}: e}$ \\
\hline \multicolumn{2}{|c|}{. Lquals: } & (3) & $18 \mathrm{~g} / \mathrm{mi}$ & . $/ \mathrm{g} / \mathrm{mi}$ & $108 \mathrm{rin}$ \\
\hline S96T002282 & 141: 1 & $\begin{array}{l}\text { Drainable } \\
\text { liquid }\end{array}$ & 96.7 & 96.5 & 96.6 \\
\hline S96T002942 & 145: 1 & $\begin{array}{l}\text { Drainable } \\
\text { liquid }\end{array}$ & 89.2 & 91.3 & 90.25 \\
\hline S96T003163 & 146: 1 & $\begin{array}{l}\text { Drainable } \\
\text { liquid }\end{array}$ & 89.4 & 84.2 & 86.8 \\
\hline
\end{tabular}


HNF-SD-WM-ER-639 Rev. 0

Table B2-20. Tank 241-U-108 Core Sample Analytical Results: Cadmium (ICP).

\begin{tabular}{|c|c|c|c|c|c|}
\hline $\begin{array}{l}\text { Sumple. } \\
\text { Kuminer. }\end{array}$ & $\begin{array}{l}\text { Sarinilo } \\
\text { I gocation }\end{array}$ & $\begin{array}{l}\text { Saroulle } \\
\text { Portion }\end{array}$ & lResult: & Buplicate & Mean \\
\hline \multicolumn{2}{|c|}{ Sollost acid digert } & (2): & $4 \mathrm{~g}_{\mathrm{g}}$ & $1.6 \mathrm{~g} g$. & 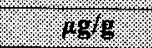 \\
\hline S96T003451 & Core 141 & $\begin{array}{l}\text { Solid } \\
\text { composite }\end{array}$ & $<4.8$ & 5.14 & $<4.97$ \\
\hline S96T003457 & Core 145 & $\begin{array}{l}\text { Solid } \\
\text { composite }\end{array}$ & $<4.95$ & $<4.84$ & $<\overline{4.895}$ \\
\hline S96T004200 & Core 146 & $\begin{array}{l}\text { Solid } \\
\text { composite }\end{array}$ & 2.95 & 4.06 & $3.505^{\mathrm{QC:a,e}}$ \\
\hline \multicolumn{2}{|c|}{ Sollitist vater digest: } & (1) & $=.998$ & $.481 \mathrm{~g}$ & $.9 \mathrm{~g} / 9$ \\
\hline S96T003453 & Core 141 & $\begin{array}{l}\text { Solid } \\
\text { composite }\end{array}$ & $<5.13$ & $<5.12$ & $<5.125$ \\
\hline S96T003662 & Core 145 & $\begin{array}{l}\text { Solid } \\
\text { composite }\end{array}$ & $<4.71$ & $<3.64$ & $<4.175^{\mathrm{QC}: \mathrm{e}}$ \\
\hline S96T004202 & Core 146 & $\begin{array}{l}\text { Solid } \\
\text { composite }\end{array}$ & $<3.08$ & $<2.75$ & $<2.915^{\text {QC:e }}$ \\
\hline \multicolumn{2}{|c|}{ (2.) Iquids } & & 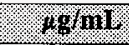 & 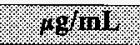 & $1.18 / \mathrm{mI}$ \\
\hline S96T002282 & 141: 1 & $\begin{array}{l}\text { Drainable } \\
\text { liquid }\end{array}$ & $<2$ & $<2$ & $<2$ \\
\hline S96T002942 & 145: 1 & $\begin{array}{l}\text { Drainable } \\
\text { liquid }\end{array}$ & $<2$ & $<2$ & $<2$ \\
\hline S96T003163 & 146: 1 & $\begin{array}{l}\text { Drainable } \\
\text { liquid }\end{array}$ & $<2$ & $<2$ & $<2$ \\
\hline
\end{tabular}


Table B2-21. Tank 241-U-108 Core Sample Analytical Results: Calcium (ICP).

\begin{tabular}{|c|c|c|c|c|c|}
\hline $\begin{array}{l}\text { Sumple. } \\
\text { Tumber. }\end{array}$ & $\begin{array}{l}\text { Sample } \\
\text { Lrocalion }\end{array}$ & $\begin{array}{l}\text { Sarnole } \\
\text { Portion }\end{array}$ & Resill & Duplicate & Morn \\
\hline \multicolumn{2}{|c|}{ S Solids acio digest: } & ?. & 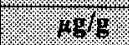 & $4 \mathrm{~g} / \mathrm{s}$ & $48 \mathrm{~g}$ \\
\hline S96T003451 & Core 141 & $\begin{array}{l}\text { Solid } \\
\text { composite }\end{array}$ & 178 & 137 & $157.5^{\mathrm{QC}: \mathrm{b}, \mathrm{e}}$ \\
\hline S96T003457 & Core 145 & $\begin{array}{l}\text { Solid } \\
\text { composite }\end{array}$ & 199 & 173 & $186^{\mathrm{QC:b,e}}$ \\
\hline S96T004200 & Core 146 & $\begin{array}{l}\text { Solid } \\
\text { composite }\end{array}$ & 109 & 137 & $123^{\mathrm{eC}: \mathrm{e}}$ \\
\hline \multicolumn{2}{|c|}{ Solidss water digest } & 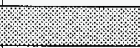 & 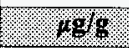 & $.48 \%$ & $98 \% 8$ \\
\hline S96T003453 & Core 141 & $\begin{array}{l}\text { Solid } \\
\text { composite }\end{array}$ & 151 & 128 & $139.5^{\mathrm{QCe}}$ \\
\hline S96T003662 & Core 145 & $\begin{array}{l}\text { Solid } \\
\text { composite }\end{array}$ & 127 & 114 & $120.5^{\mathrm{QC:e}}$ \\
\hline S96T004202 & Core 146 & $\begin{array}{l}\text { Solid } \\
\text { composite }\end{array}$ & 88.5 & 82.6 & 85.55 \\
\hline \multicolumn{2}{|c|}{ Tiguads } & 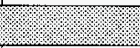 & . $18 \mathrm{gial}$ & $.89 \mathrm{gri}$ & (1) \\
\hline S96T002282 & 141: 1 & $\begin{array}{l}\text { Drainable } \\
\text { liquid }\end{array}$ & 41.9 & $<40.1$ & $<41$ \\
\hline S96T002942 & 145: 1 & $\begin{array}{l}\text { Drainable } \\
\text { liquid }\end{array}$ & $<40.1$ & $<40.1$ & $<40.1$ \\
\hline S96T003163 & 146: 1 & $\begin{array}{l}\text { Drainable } \\
\text { liquid }\end{array}$ & 41 & $<40.1$ & $<40.55$ \\
\hline
\end{tabular}


HNF-SD-WM-ER-639 Rev. 0

Table B2-22. Tank 241-U-108 Core Sample Analytical Results: Chromium (ICP). (3 sheets)

\begin{tabular}{|c|c|c|c|c|c|}
\hline $\begin{array}{l}\text { Sample } \\
\text { Vumibe; }\end{array}$ & $\begin{array}{l}\text { Samile } \\
\text { Locition }\end{array}$ & $\begin{array}{l}\text { Sainple } \\
\text { Prortion. }\end{array}$ & aresilit & miplicute & . nean \\
\hline \multicolumn{2}{|c|}{ 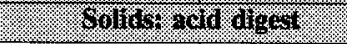 } & & 1998 & 1898 & 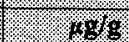 \\
\hline S96T003451 & Core 141 & $\begin{array}{l}\text { Solid } \\
\text { composite }\end{array}$ & 4,060 & 3,930 & 3,995 \\
\hline S96T003457 & Core 145 & $\begin{array}{l}\text { Solid } \\
\text { composite }\end{array}$ & 3,630 & 3,610 & 3,620 \\
\hline S96T004200 & Core 146 & $\begin{array}{l}\text { Solid } \\
\text { composite }\end{array}$ & 3,430 & 4,300 & $3,865^{\mathrm{QC} c \mathrm{e}}$ \\
\hline \multicolumn{2}{|c|}{ Sollder hision } & & 198 & $10 \mathrm{~g} / \mathrm{g}$ & 18968 \\
\hline S96T002257 & $141: 1$ & Lower $1 / 2$ & 12,600 & 9,310 & $10,960^{\mathrm{QC:e}}$ \\
\hline S96T002258 & 141: 2 & Upper $1 / 2$ & 3,630 & 3,650 & 3,640 \\
\hline S96T002259 & 141: 3 & Upper $1 / 2$ & 2,900 & 3,000 & 2,950 \\
\hline S96T002260 & 141: $3 A$ & Upper $1 / 2$ & 2,500 & 2,660 & 2,580 \\
\hline S96T002261 & \multirow[t]{2}{*}{ 141: 4} & Upper $1 / 2$ & 4,810 & 4,430 & 4,620 \\
\hline S96T002262 & & Lower $1 / 2$ & 5,100 & 5,300 & 5,200 \\
\hline S96T002263 & \multirow[t]{2}{*}{$141: 4 \mathrm{~A}$} & Upper $1 / 2$ & 5,390 & 5,420 & 5,405 \\
\hline S96T002264 & & Lower $1 / 2$ & 4,380 & 4,230 & 4,305 \\
\hline S96T002608 & \multirow[t]{2}{*}{ 141: 5} & Upper $1 / 2$ & 4,670 & 4,490 & 4,580 \\
\hline S96T002609 & & Lower $1 / 2$ & 3,320 & 3,210 & 3,265 \\
\hline S96T002610 & \multirow[t]{2}{*}{$141: 6$} & Upper $1 / 2$ & 2,920 & 2,830 & 2,875 \\
\hline S96T002611 & & Lower $1 / 2$ & 2,360 & 2,530 & 2,445 \\
\hline S96T002612 & \multirow[t]{2}{*}{ 141: 7} & Upper $1 / 2$ & 5,300 & 5,770 & 5,535 \\
\hline S96T002613 & & Lower $1 / 2$ & 4,600 & 4,470 & 4,535 \\
\hline S96T002614 & \multirow[t]{2}{*}{ 141: 8} & Upper $1 / 2$ & 6,300 & 6,080 & 6,190 \\
\hline S96T002615 & & Lower $1 / 2$ & 5,650 & 5,740 & 5,695 \\
\hline S96T002616 & 141: 9 & Upper $1 / 2$ & 937 & 918 & 927.5 \\
\hline S96T002909 & 145: 2 & Upper $1 / 2$ & 2,390 & 3,000 & $2,695^{\mathrm{QC}: e}$ \\
\hline S96T002910 & \multirow[t]{2}{*}{$145: 3$} & Upper $1 / 2$ & 3,700 & 3,160 & $3,430^{\mathrm{QC}: \mathrm{e}}$ \\
\hline S96T002911 & & Lower $1 / 2$ & 3,370 & 3,230 & 3,300 \\
\hline S96T002912 & \multirow[t]{2}{*}{ 145: 4} & Upper $1 / 2$ & 2,770 & 2,900 & 2,835 \\
\hline S96T002913 & & Lower $1 / 2$ & 3,790 & 3,660 & 3,725 \\
\hline S96T002914 & \multirow[t]{2}{*}{ 145: 5} & Upper $1 / 2$ & 3,070 & 3,320 & 3,195 \\
\hline S96T002915 & & Lower $1 / 2$ & 5,940 & 6,490 & 6,215 \\
\hline S96T002916 & \multirow[t]{2}{*}{$145: 6$} & Upper $1 / 2$ & 3,950 & 3,720 & 3,835 \\
\hline S96T002917 & & Lower $1 / 2$ & 3,340 & 3,310 & 3,325 \\
\hline
\end{tabular}


Table B2-22. Tank 241-U-108 Core Sample Analytical Results: Chromium (ICP). (3 sheets)

\begin{tabular}{|c|c|c|c|c|c|}
\hline $\begin{array}{l}\text { Simple } \\
\text { Numiser }\end{array}$ & $\begin{array}{l}\text { Gimples } \\
\text { Locition }\end{array}$ & $\begin{array}{l}\text { Sample: } \\
\text { romitun. }\end{array}$ & ficsilit & Buplicate & Mear \\
\hline \multicolumn{2}{|c|}{ 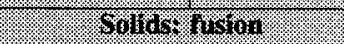 } & (3. & .898 & .486 & $1.98 \%$ \\
\hline S96T002918 & \multirow[t]{2}{*}{$145: 7$} & Upper $1 / 2$ & 3,960 & 3,980 & 3,970 \\
\hline S96T002919 & & Lower $1 / 2$ & 5,060 & 5,200 & 5,130 \\
\hline S96T002920 & \multirow[t]{2}{*}{$145: 8$} & Upper $1 / 2$ & 2,820 & 2,850 & 2,835 \\
\hline S96T002921 & & Lower $1 / 2$ & 3,940 & 3,820 & 3,880 \\
\hline S96T002922 & \multirow[t]{2}{*}{ 145: 9} & Upper $1 / 2$ & 7,890 & 7,960 & 7,925 \\
\hline S96T002923 & & Lower $1 / 2$ & 2,390 & 2,480 & 2,435 \\
\hline S96T003143 & 146: 1 & Upper $1 / 2$ & 3,520 & 3,870 & 3,695 \\
\hline S96T003144 & \multirow[t]{2}{*}{ 146: 2} & Upper $1 / 2$ & 3,380 & 3,500 & 3,440 \\
\hline S96T003145 & & Lower $1 / 2$ & 1,680 & 1,540 & 1,610 \\
\hline S96T003146 & \multirow[t]{2}{*}{ 146: 3} & Upper $1 / 2$ & 3,200 & 3,360 & 3,280 \\
\hline S96T003147 & & Lower $1 / 2$ & 1,290 & 1,330 & 1,310 \\
\hline S96T003001 & 146: $3 \mathrm{~A}$ & Upper $1 / 2$ & 2,940 & 2,900 & 2,920 \\
\hline S96T003002 & \multirow[t]{2}{*}{ 146: 4} & Upper $1 / 2$ & 3,400 & 3,390 & 3,395 \\
\hline S96T003003 & & Lower $1 / 2$ & 4,890 & 4,940 & 4,915 \\
\hline S96T003004 & \multirow[t]{2}{*}{$146: 5$} & Upper $1 / 2$ & 5,100 & 4,810 & 4,955 \\
\hline S96T003005 & & Lower $1 / 2$ & 3,650 & 3,280 & $3,465^{\mathrm{QC}: \mathrm{e}}$ \\
\hline S96T003006 & \multirow[t]{2}{*}{ 146: 6} & Upper $1 / 2$ & 3,520 & 3,990 & $3,755^{Q C: e}$ \\
\hline S96T003007 & & Lower $1 / 2$ & 3,970 & 3,850 & 3,910 \\
\hline S96T003008 & \multirow[t]{2}{*}{ 146: 7} & Upper $1 / 2$ & 3,990 & 4,350 & 4,170 \\
\hline S96T003009 & & Lower $1 / 2$ & 5,350 & 5,140 & 5,245 \\
\hline S96T003148 & \multirow[t]{2}{*}{ 146: 8} & Upper $1 / 2$ & 4,200 & 4,790 & $4,495^{\mathrm{QC}: \mathrm{e}}$ \\
\hline S96T003149 & & Lower $1 / 2$ & 4,890 & 5,070 & 4,980 \\
\hline S96T004180 & \multirow[t]{2}{*}{ 146: 9} & Lower $1 / 2$ & 6,300 & 6,510 & 6,405 \\
\hline S96T003150 & & Upper $1 / 2$ & 6,590 & 7,010 & 6,800 \\
\hline S96T003450 & Core 141 & $\begin{array}{l}\text { Solid } \\
\text { composite }\end{array}$ & 4,310 & 3,810 & $4,060^{\mathrm{QC}: \mathrm{e}}$ \\
\hline S96T003660 & Core 145 & $\begin{array}{l}\text { Solid } \\
\text { composite }\end{array}$ & 5,050 & 3,150 & $4,100^{\mathrm{QC}: \mathrm{e}}$ \\
\hline S96T004199 & Core 146 & $\begin{array}{l}\text { Solid } \\
\text { composite }\end{array}$ & 4,090 & 3,950 & 4,020 \\
\hline
\end{tabular}


Table B2-22. Tank 241-U-108 Core Sample Analytical Results: Chromium (ICP). (3 sheets)

\begin{tabular}{|c|c|c|c|c|c|}
\hline $\begin{array}{l}\text { Simple } \\
\text { Yumber. }\end{array}$ & Somple & $\begin{array}{l}\text { Sample } \\
\text { Portion }\end{array}$ & Reswilt & Diplicate & Mean. \\
\hline \multicolumn{3}{|c|}{ Soluts wate diget } & $=148$ & $108 \%$ & 1. $.1 .496 \mathrm{~s}$ \\
\hline S96T003453 & Core 141 & $\begin{array}{l}\text { Solid } \\
\text { composite }\end{array}$ & 321 & 317 & 319 \\
\hline S96T003662 & Core 145 & $\begin{array}{l}\text { Solid } \\
\text { composite }\end{array}$ & 287 & $27 \overline{3}$ & 280 \\
\hline S96T004202 & Core 146 & $\begin{array}{l}\text { Solid } \\
\text { composite }\end{array}$ & 462 & $\overline{474}$ & 468 \\
\hline \multicolumn{3}{|c|}{ 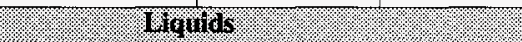 } & OWIIII & kg/ni. & $.98 \mathrm{mi}$ \\
\hline S96T002282 & 141: 1 & $\begin{array}{l}\text { Drainable } \\
\text { liquid }\end{array}$ & 1,230 & 1,250 & 1,240 \\
\hline S96T002942 & 145: 1 & $\begin{array}{l}\text { Drainable } \\
\text { liquid }\end{array}$ & 1,130 & 1,130 & 1,130 \\
\hline S96T003163 & 146: 1 & $\begin{array}{l}\text { Drainable } \\
\text { liquid }\end{array}$ & 1,500 & 1,420 & 1,460 \\
\hline
\end{tabular}


Table B2-23. Tank 241-U-108 Core Sample Analytical Results: Copper (ICP).

\begin{tabular}{|c|c|c|c|c|c|}
\hline Sample & Sampie. & Somple & Revif & Buplicate & Then \\
\hline \multicolumn{3}{|c|}{ - Solfor and digest } & 488 & .1689 & $169 \%$ \\
\hline S96T003451 & Core 141 & $\begin{array}{l}\text { Solid } \\
\text { composite }\end{array}$ & $<9.6$ & $<9.63$ & $<9.615$ \\
\hline S96T003457 & Core 145 & $\begin{array}{l}\text { Solid } \\
\text { composite }\end{array}$ & $<9.89$ & $<9.67$ & $<9 . \overline{78}$ \\
\hline S96T004200 & Core 146 & $\begin{array}{l}\text { Solid } \\
\text { composite }\end{array}$ & 5.77 & $<5.25$ & $<5.51$ \\
\hline \multicolumn{2}{|c|}{ Solliks rater olgert } & & $14 \mathrm{~g} / 8$ & .7868 & $10 \mathrm{~g} 8$ \\
\hline S96T003453 & Core 141 & $\begin{array}{l}\text { Solid } \\
\text { composite }\end{array}$ & $<10.3$ & $<10.2$ & $<10.25$ \\
\hline S96T003662 & Core 145 & $\begin{array}{l}\text { Solid } \\
\text { composite }\end{array}$ & $<9.42$ & $<7.28$ & $<8.35^{\mathrm{QC}: \mathrm{e}}$ \\
\hline S96T004202 & Core 146 & $\begin{array}{l}\text { Solid } \\
\text { composite }\end{array}$ & $<6.17$ & $<5.5$ & $<5.835^{Q \mathrm{QC}: \mathrm{e}}$ \\
\hline \multicolumn{2}{|c|}{ Tiquidis } & (2) & (1610 & $48 \%$ & 1 go/firl \\
\hline S96T002282 & 141: 1 & $\begin{array}{l}\text { Drainable } \\
\text { liquid }\end{array}$ & $<4.01$ & $<4.01$ & $<4.01$ \\
\hline S96T002942 & $145: 1$ & $\begin{array}{l}\text { Drainable } \\
\text { liquid }\end{array}$ & $<4.01$ & $<4.01$ & $<4.01$ \\
\hline S96T003163 & 146: 1 & $\begin{array}{l}\text { Drainable } \\
\text { liquid }\end{array}$ & $<4.01$ & $<4.01$ & $<4.01$ \\
\hline
\end{tabular}


Table B2-24. Tank 241-U-108 Core Sample Analytical Results: Iron (ICP). (3 sheets)

\begin{tabular}{|c|c|c|c|c|c|}
\hline Somol & Kacrinom & W11010 & thervil & Binplicate & Hean \\
\hline \multicolumn{3}{|c|}{ 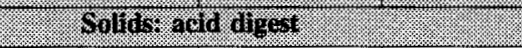 } & 28\%s & .1096 & 48 \\
\hline S96T003451 & Core 141 & $\begin{array}{l}\text { Solid } \\
\text { composite }\end{array}$ & 176 & 147 & $161.5^{Q C: e}$ \\
\hline \$96T003457 & Core 145 & $\begin{array}{l}\text { Solid } \\
\text { composite }\end{array}$ & 235 & 179 & $207^{\overline{\mathrm{QC}: \mathrm{e}}}$ \\
\hline S96T004200 & Core 146 & $\begin{array}{l}\text { Solid } \\
\text { composite }\end{array}$ & 144 & 171 & $157.5^{\mathrm{QC} e}$ \\
\hline \multicolumn{2}{|c|}{. Oolid Gision } & & 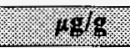 & 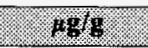 & (.). \\
\hline S96T002257 & 141: 1 & Lower $1 / 2$ & $<893$ & $<895$ & $<894$ \\
\hline S96T002258 & 141: 2 & Upper $1 / 2$ & $<929$ & $<967$ & $<948$ \\
\hline S96T002259 & $141: 3$ & Upper $1 / 2$ & $<878$ & $<864$ & $<871$ \\
\hline S96T002260 & $141: 3 \mathrm{~A}$ & Upper $1 / 2$ & $<978$ & $<941$ & $<9 \overline{959.5}$ \\
\hline S96T002261 & \multirow[t]{2}{*}{$141: 4$} & Upper $1 / 2$ & $<913$ & $<907$ & $<910$ \\
\hline S96T002262 & & Lower $1 / 2$ & $<\overline{991}$ & $<945$ & $<968$ \\
\hline S96T002263 & \multirow[t]{2}{*}{$141: 4 \mathrm{~A}$} & Upper $1 / 2$ & $<904$ & $<925$ & $<914.5$ \\
\hline S96T002264 & & Lower $1 / 2$ & $<904$ & $<893$ & $<898.5$ \\
\hline S96T002608 & \multirow[t]{2}{*}{$141: 5$} & Upper $1 / 2$ & $<9 \overline{44}$ & $<954$ & $<\overline{949}$ \\
\hline S96T002609 & & Lower $1 / 2$ & $<1,100$ & $<1,020$ & $<1,060$ \\
\hline S96T002610 & \multirow[t]{2}{*}{ 141: 6} & Upper $1 / 2$ & $<1,060$ & $<963$ & $<1,011$ \\
\hline S96T002611 & & Lower $1 / 2$ & $<1,100$ & $<1,120$ & $<1,110$ \\
\hline S96T002612 & \multirow[t]{2}{*}{$141: 7$} & Upper $1 / 2$ & $<\overline{947}$ & $<968$ & $<957.5$ \\
\hline S96T002613 & & Lower $1 / 2$ & $<1,100$ & $<1,060$ & $<\overline{1,080}$ \\
\hline S96T002614 & \multirow[t]{2}{*}{ 141: 8} & Upper $1 / 2$ & $<1,030$ & $<934$ & $<982$ \\
\hline \$96T002615 & & Lower $1 / 2$ & $<1,080$ & $<973$ & $<1,026^{\mathrm{QC:e}}$ \\
\hline S96T002616 & 141: 9 & Upper $1 / 2$ & $<1,080$ & $<1,060$ & $<1, \overline{070}$ \\
\hline S96T002909 & $145: 2$ & Upper $1 / 2$ & 1,070 & $<970$ & $<1,020$ \\
\hline S96T002910 & \multirow[t]{2}{*}{$145: 3$} & Upper $1 / 2$ & $<1,010$ & $<1,010$ & $<1,010$ \\
\hline S96T002911 & & Lower $1 / 2$ & $<1,010$ & $<1,020$ & $<1, \overline{015}$ \\
\hline S96T002912 & \multirow[t]{2}{*}{ 145: 4} & Upper $1 / 2$ & $<1,110$ & $<1,120$ & $<1,115$ \\
\hline S96T002913 & & Lower $1 / 2$ & $<1,050$ & $<1,090$ & $<1,070$ \\
\hline S96T002914 & \multirow[t]{2}{*}{$145: 5$} & Upper $1 / 2$ & $<1,110$ & $<1,090$ & $<1,100$ \\
\hline S96T002915 & & Lower $1 / 2$ & $<1,100$ & $<1,110$ & $<1,105$ \\
\hline S96T002916 & \multirow[t]{2}{*}{ 145: 6} & Upper $1 / 2$ & $<1,040$ & $<1,050$ & $<1,045$ \\
\hline$\overline{\text { S96T002917 }}$ & & Lower $1 / 2$ & $<1,000$ & $<990$ & $<995$ \\
\hline
\end{tabular}


Table B2-24. Tank 241-U-108 Core Sample Analytical Results: Iron (ICP). (3 sheets)

\begin{tabular}{|c|c|c|c|c|c|}
\hline ofmor & ogarng & 301101 & $1 \mathrm{tr} s \mathrm{H}$ & Buplicale & Mern \\
\hline S96T002918 & \multirow[t]{2}{*}{ 145: 7} & Upper $1 / 2$ & $<1,040$ & $<1,030$ & $<1,035$ \\
\hline S96T002919 & & Lower $1 / 2$ & $<1,050$ & $<1,040$ & $<1,045$ \\
\hline S96T002920 & \multirow[t]{2}{*}{ 145: 8} & Upper $1 / 2$ & $<983$ & $<967$ & $<975$ \\
\hline S96T002921 & & Lower $1 / 2$ & $<1,020$ & $<1,020$ & $<1,020$ \\
\hline S96T002922 & \multirow[t]{2}{*}{$145: 9$} & Upper $1 / 2$ & 1,310 & $1, \overline{440}$ & 1,375 \\
\hline S96T002923 & & Lower $1 / 2$ & $<982$ & $<984$ & $<983$ \\
\hline \$96T003143 & $146: 1$ & Upper $1 / 2$ & $<1,120$ & $<1,130$ & $<1,125$ \\
\hline S96T003144 & \multirow[t]{2}{*}{ 146: 2} & Upper $1 / 2$ & $<1,090$ & $<1,050$ & $<1,070$ \\
\hline S96T003145 & & Lower $1 / 2$ & $<934$ & $<1,130$ & $<1,032^{\mathrm{QC}: \mathrm{e}}$ \\
\hline S96T003146 & \multirow[t]{2}{*}{ 146: 3} & Upper $1 / 2$ & $<1,110$ & $<1,130$ & $<1,120$ \\
\hline S96T003147 & & Lower $1 / 2$ & $<961$ & $<1,020$ & $<990.5$ \\
\hline \$96T003001 & 146: $3 A$ & Upper $1 / 2$ & $<1,060$ & $<1,080$ & $<1,070$ \\
\hline S96T003002 & \multirow[t]{2}{*}{$146: 4$} & Upper $1 / 2$ & $<1,010$ & $<1,060$ & $<1,035$ \\
\hline S96T003003 & & Lower $1 / 2$ & $<976$ & $<1,060$ & $<1,018$ \\
\hline S96T003004 & \multirow[t]{2}{*}{ 146: 5} & Upper $1 / 2$ & $<1,100$ & $<969$ & $<1,034^{\mathrm{QC}: \mathrm{e}}$ \\
\hline S96T003005 & & Lower $1 / 2$ & $<1,050$ & $<990$ & $<1, \overline{020}$ \\
\hline S96T003006 & \multirow[t]{2}{*}{ 146: 6} & Upper $1 / 2$ & $<1,130$ & $<954$ & $<1,042^{\mathrm{QC}: \mathrm{e}}$ \\
\hline S96T003007 & & Lower $1 / 2$ & $<1,020$ & $<1,030$ & $<1,025$ \\
\hline S96T003008 & \multirow[t]{2}{*}{ 146: 7} & Upper $1 / 2$ & $<978$ & $<1,020$ & $<999$ \\
\hline S96T003009 & & Lower $1 / 2$ & $<932$ & $<1,110$ & $<1,021^{\mathrm{QC}: \mathrm{e}}$ \\
\hline S967003148 & \multirow[t]{2}{*}{ 146: 8} & Upper $1 / 2$ & $<\overline{965}$ & $<982$ & $<973.5$ \\
\hline S96T003149 & & Lower $1 / 2$ & $<982$ & $<946$ & $<964$ \\
\hline S96T004180 & \multirow[t]{2}{*}{ 146: 9} & Lower $1 / 2$ & 1,360 & 1,400 & 1,380 \\
\hline S96T003150 & & Upper $1 / 2$ & $<1,140$ & $<1,070$ & $<1,105$ \\
\hline S96T003450 & Core 141 & $\begin{array}{l}\text { Solid } \\
\text { composite }\end{array}$ & $<987$ & $<1,010$ & $<998.5$ \\
\hline S96T003660 & Core 145 & $\begin{array}{l}\text { Solid } \\
\text { composite }\end{array}$ & $<987$ & $<781$ & $<884^{Q C: e}$ \\
\hline S96T004199 & Core 146 & $\begin{array}{l}\text { Solid } \\
\text { composite }\end{array}$ & $<1,010$ & $<981$ & $<995.5$ \\
\hline
\end{tabular}


Table B2-24. Tank 241-U-108 Core Sample Analytical Results: Iron (ICP). (3 sheets)

\begin{tabular}{|c|c|c|c|c|c|}
\hline Sample & 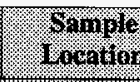 & $\begin{array}{l}\text { Saruplo } \\
\text { Iortion }\end{array}$ & Resull & Biplierse & Mean \\
\hline \multicolumn{2}{|c|}{ Wnith water aliest } & ?.: & 989 & Hgig & 14848 \\
\hline S96T003453 & Core 141 & $\begin{array}{l}\text { Solid } \\
\text { composite }\end{array}$ & $<51.3$ & $<51.2$ & $<51.25$ \\
\hline S96T003662 & Core 145 & $\begin{array}{l}\text { Solid } \\
\text { composite }\end{array}$ & $<47.1$ & $<36.4$ & $<41.75^{\mathrm{QC}: \mathrm{e}}$ \\
\hline S96T004202 & Core 146 & $\begin{array}{l}\text { Solid } \\
\text { composite }\end{array}$ & $<30.8$ & $<27.5$ & $<29.15^{\text {QC:e }}$ \\
\hline \multicolumn{2}{|c|}{ Giginits: } & 1. & 4.48101 & 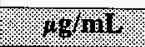 & $.46 \mathrm{~m}$. \\
\hline S96T002282 & 141: 1 & $\begin{array}{l}\text { Drainable } \\
\text { liquid }\end{array}$ & 29.2 & 29.2 & 29.2 \\
\hline S96T002942 & $145: 1$ & $\begin{array}{l}\text { Drainable } \\
\text { liquid }\end{array}$ & 28.9 & 29.1 & 29 \\
\hline S96T003163 & 146: 1 & $\begin{array}{l}\text { Drainable } \\
\text { liquid }\end{array}$ & 35.7 & 34.4 & 35.05 \\
\hline
\end{tabular}

Table B2-25. Tank 241-U-108 Grab Sample Analytical Results: Iron (ICP).

\begin{tabular}{|c|c|c|c|c|c|}
\hline $\begin{array}{l}\text { Sumple } \\
\text { Trumlet }\end{array}$ & $\begin{array}{l}\text { Shmivi } \\
\text { Uocation }\end{array}$ & $\begin{array}{l}\text { Sirmple } \\
\text { rortion }\end{array}$ & Resin & Bunlugue & Mean \\
\hline \multicolumn{2}{|c|}{ IFiquals } & 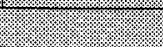 & 8.8611 & $49 \mathrm{mi}$ & $7.9 / 111$ \\
\hline S95T000978 & Riser 7 & Grab sample & 43.24 & 36.95 & 40.10 \\
\hline
\end{tabular}

Table B2-26. Tank 241-U-108 Core Sample Analytical Results: Lithium (ICP).

\begin{tabular}{|c|c|c|c|c|c|}
\hline $\begin{array}{l}\text { Sample } \\
\text { Vumile? }\end{array}$ & $\begin{array}{l}\text { Somple } \\
\text { Locontion }\end{array}$ & $\begin{array}{l}\text { Somple } \\
\text { Tortion }\end{array}$ & Pesral & Oyilicaro. & Mean \\
\hline \multicolumn{2}{|c|}{ 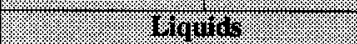 } & 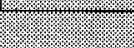 & 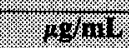 & . & 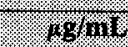 \\
\hline S96T002282 & 141: 1 & $\begin{array}{l}\text { Drainable } \\
\text { liquid }\end{array}$ & $<4.01$ & $<4.01$ & $<4.01$ \\
\hline S96T002942 & 145: 1 & $\begin{array}{l}\text { Drainable } \\
\text { liquid }\end{array}$ & $<4.01$ & $<4.01$ & $<4.01$ \\
\hline S96T003163 & 146: 1 & $\begin{array}{l}\text { Drainable } \\
\text { liquid }\end{array}$ & 4.09 & $<4.01$ & $<4.05$ \\
\hline
\end{tabular}


Table B2-27. Tank 241-U-108 Core Sample Analytical Results: Manganese (ICP). (3 sheets)

\begin{tabular}{|c|c|c|c|c|c|}
\hline Somple & $\begin{array}{l}\text { Somples } \\
\text { Locorious }\end{array}$ & $\begin{array}{l}\text { Sample } \\
\text { Portion }\end{array}$ & Irowil & Bupliente. & Meran \\
\hline \multicolumn{3}{|c|}{ 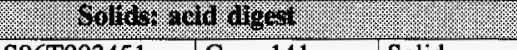 } & 4.98 & 1.986 & 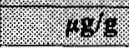 \\
\hline S96T003451 & Core 141 & $\begin{array}{l}\text { Solid } \\
\text { composite }\end{array}$ & 45.3 & 45.3 & 45.3 \\
\hline S96T003457 & Core 145 & $\begin{array}{l}\text { Solid } \\
\text { composite }\end{array}$ & 77.7 & 77 & 77.35 \\
\hline S96T004200 & Core 146 & $\begin{array}{l}\text { Solid } \\
\text { composite }\end{array}$ & 68.4 & 73.6 & $71^{\mathrm{QC}: \mathrm{a}}$ \\
\hline \multicolumn{2}{|c|}{ 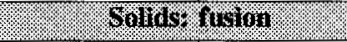 } & & 1.88 & $.69 \mathrm{~g}$ & 188 \\
\hline S96T002257 & 141: 1 & Lower $1 / 2$ & $<179$ & $<179$ & $<179$ \\
\hline S96T002258 & 141: 2 & Upper $1 / 2$ & $<186$ & $<193$ & $<189.5$ \\
\hline S96T002259 & $141: 3$ & Upper $1 / 2$ & $<176$ & $<173$ & $<174.5$ \\
\hline S96T002260 & 141: $3 \mathrm{~A}$ & Upper $1 / 2$ & $<196$ & $<188$ & $<192$ \\
\hline S96T002261 & \multirow[t]{2}{*}{$141: 4$} & Upper $1 / 2$ & $<183$ & $<181$ & $<182$ \\
\hline S96T002262 & & Lower $1 / 2$ & $<198$ & $<189$ & $<193.5$ \\
\hline S96T002263 & \multirow[t]{2}{*}{ 141: $4 \mathrm{~A}$} & Upper $1 / 2$ & $<181$ & $<185$ & $<183$ \\
\hline S96T002264 & & Lower $1 / 2$ & $<181$ & $<179$ & $<180$ \\
\hline S96T002608 & \multirow[t]{2}{*}{$141: 5$} & Upper $1 / 2$ & $<189$ & $<191$ & $<190$ \\
\hline S96T002609 & & Lower $1 / 2$ & $<221$ & $<205$ & $<213$ \\
\hline S96T002610 & \multirow[t]{2}{*}{$141: 6$} & Upper $1 / 2$ & $<212$ & $<193$ & $<202.5$ \\
\hline S96T002611 & & Lower $1 / 2$ & $<219$ & $<224$ & $<221.5$ \\
\hline S96T002612 & \multirow[t]{2}{*}{ 141: 7} & Upper $1 / 2$ & $<189$ & $<194$ & $<191.5$ \\
\hline S96T002613 & & Lower $1 / 2$ & $<221$ & $<212$ & $<216.5$ \\
\hline S96T002614 & \multirow[t]{2}{*}{$141: 8$} & Upper $1 / 2$ & $<205$ & $<187$ & $<196$ \\
\hline S96T002615 & & Lower $1 / 2$ & $<215$ & 198 & $<206.5$ \\
\hline S96T002616 & 141: 9 & Upper $1 / 2$ & $<216$ & $<212$ & $<\overline{214}$ \\
\hline S96T002909 & $145: 2$ & Upper $1 / 2$ & $<196$ & $<194$ & $<\overline{195}$ \\
\hline S96T002910 & \multirow[t]{2}{*}{$145: 3$} & Upper $1 / 2$ & $<202$ & $<20 \overline{2}$ & $<202$ \\
\hline S96T002911 & & Lower $1 / 2$ & $<202$ & $<203$ & $<202.5$ \\
\hline S96T002912 & \multirow[t]{2}{*}{ 145: 4} & Upper $1 / 2$ & $<223$ & $<223$ & $<223$ \\
\hline S96T002913 & & Lower $1 / 2$ & $<211$ & $<218$ & $<214.5$ \\
\hline S96T002914 & \multirow[t]{2}{*}{$145: 5$} & Upper $1 / 2$ & $<221$ & $<219$ & $<220$ \\
\hline S96T002915 & & Lower $1 / 2$ & $<220$ & $<221$ & $<220.5$ \\
\hline S96T002916 & \multirow[t]{2}{*}{$145: 6$} & Upper $1 / 2$ & $<209$ & $<210$ & $<209.5$ \\
\hline S96T002917 & & Lower $1 / 2$ & $<201$ & $<198$ & $<199.5$ \\
\hline
\end{tabular}


Table B2-27. Tank 241-U-108 Core Sample Analytical Results: Manganese (ICP). ( 3 sheets)

\begin{tabular}{|c|c|c|c|c|c|}
\hline 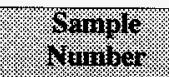 & $\begin{array}{l}\text { Sample } \\
\text { Yogarisan }\end{array}$ & $\begin{array}{l}\text { Sample } \\
\text { remtion }\end{array}$ & resalti & Duplicate & Mean \\
\hline \multicolumn{2}{|c|}{ 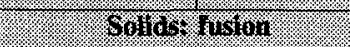 } & 1.8 .2 & 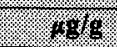 & 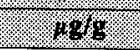 & $16 \mathrm{~g} / \mathrm{g}$ \\
\hline $96 \mathrm{~T} 002918$ & \multirow[t]{2}{*}{$145: 7$} & Upper $1 / 2$ & $<207$ & $<206$ & $<206.5$ \\
\hline S96T002919 & & Lower $1 / 2$ & $<211$ & $<207$ & $<209$ \\
\hline S96T002920 & \multirow[t]{2}{*}{ 145: 8} & Upper $1 / 2$ & $<197$ & $<193$ & $<195$ \\
\hline S96T002921 & & Lower $1 / 2$ & $<204$ & $<205$ & $<204.5$ \\
\hline S96T002922 & \multirow[t]{2}{*}{ 145: 9} & Upper $1 / 2$ & 671 & 712 & 691.5 \\
\hline S96T002923 & & Lower $1 / 2$ & $<196$ & $<197$ & $<196.5$ \\
\hline S96T003143 & 146: 1 & Upper $1 / 2$ & $<224$ & $<225$ & $<224.5$ \\
\hline S96T003144 & \multirow[t]{2}{*}{$146: 2$} & Upper $1 / 2$ & $<218$ & $<210$ & $<214$ \\
\hline S96T003145 & & Lower $1 / 2$ & $<187$ & $<227$ & $<207^{\mathrm{QC} ; \mathrm{e}}$ \\
\hline S96T003146 & \multirow[t]{2}{*}{$146: 3$} & Upper $1 / 2$ & $<222$ & $<226$ & $<224$ \\
\hline S96T003147 & & Lower $1 / 2$ & $<192$ & $<203$ & $<197.5$ \\
\hline S96T003001 & 146: $3 A$ & Upper $1 / 2$ & $<211$ & $<216$ & $<213.5$ \\
\hline S96T003002 & \multirow[t]{2}{*}{ 146: 4} & Upper $1 / 2$ & $<202$ & $<213$ & $<207.5$ \\
\hline S96T003003 & & Lower $1 / 2$ & $<195$ & $<212$ & $<203.5$ \\
\hline S96T003004 & \multirow[t]{2}{*}{ 146: 5} & Upper $1 / 2$ & $<219$ & $<194$ & $<206.5^{\mathrm{QC}: \mathrm{c}}$ \\
\hline S96T003005 & & Lower $1 / 2$ & $<209$ & $<198$ & $<203.5$ \\
\hline S96T003006 & \multirow[t]{2}{*}{$146: 6$} & Upper $1 / 2$ & $<226$ & $<191$ & $<208.5^{\mathrm{QC}: \mathrm{e}}$ \\
\hline S96T003007 & & Lower $1 / 2$ & $<204$ & $<206$ & $<205$ \\
\hline S96T003008 & \multirow[t]{2}{*}{$146: 7$} & Upper $1 / 2$ & $<196$ & $<203$ & $<199.5$ \\
\hline S96T003009 & & Lower $1 / 2$ & $<186$ & $<223$ & $<204.5^{Q C: e}$ \\
\hline S96T003148 & \multirow[t]{2}{*}{$146: 8$} & Upper $1 / 2$ & $<193$ & $<196$ & $<194.5$ \\
\hline S96T003149 & & Lower $1 / 2$ & $<196$ & $<189$ & $<192.5$ \\
\hline S96T004180 & \multirow[t]{2}{*}{ 146: 9} & Lower $1 / 2$ & 785 & 823 & 804 \\
\hline S96T003150 & & Upper $1 / 2$ & 455 & 474 & 464.5 \\
\hline S96T003450 & Core 141 & $\begin{array}{l}\text { Solid } \\
\text { composite }\end{array}$ & $<197$ & $<\overline{202}$ & $<199.5$ \\
\hline S96T003660 & Core 145 & $\begin{array}{l}\text { Solid } \\
\text { composite }\end{array}$ & $<197$ & $<156$ & $<176.5^{\mathrm{QC}: \mathrm{e}}$ \\
\hline S96T004199 & Core 146 & $\begin{array}{l}\text { Solid } \\
\text { composite }\end{array}$ & $<202$ & $<196$ & $<199$ \\
\hline
\end{tabular}


Table B2-27. Tank 241-U-108 Core Sample Analytical Results: Manganese (ICP). (3 sheets)

\begin{tabular}{|c|c|c|c|c|c|}
\hline $\begin{array}{l}\text { Simple. } \\
\text { Ninatior. }\end{array}$ & $\begin{array}{l}\text { Sample } \\
\text { I bezilion }\end{array}$ & $\begin{array}{l}\text { Sample } \\
\text { gotion }\end{array}$ & ressint & Buplicate & Mean \\
\hline \multicolumn{3}{|c|}{ Sollow water alger } & $4.4 \mathrm{gg}$ & $1.18 \%$ & $148 \%$ \\
\hline S96T003453 & Core 141 & $\begin{array}{l}\text { Solid } \\
\text { composite }\end{array}$ & $<10.3$ & $<10.2$ & $<10.25$ \\
\hline S96T003662 & Core 145 & $\begin{array}{l}\text { Solid } \\
\text { composite }\end{array}$ & $<9.42$ & $<7.28$ & $<8.35^{\mathrm{QCe}}$ \\
\hline S96T004202 & Core 146 & $\begin{array}{l}\text { Solid } \\
\text { composite }\end{array}$ & $<6.17$ & $<5.5$ & $<5.84^{\mathrm{QC} e \mathrm{e}}$ \\
\hline \multicolumn{3}{|c|}{ 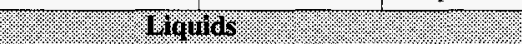 } & mg/nil. & $.4 \mathrm{~g} / \mathrm{m}$ & . $18 \mathrm{mi}$ \\
\hline S96T002282 & 141: 1 & $\begin{array}{l}\text { Drainable } \\
\text { liquid }\end{array}$ & $<4.01$ & $<4.01$ & $<4.01$ \\
\hline S96T002942 & 145: 1 & $\begin{array}{l}\text { Drainable } \\
\text { liquid }\end{array}$ & $<4.01$ & $<4.01$ & $<\overline{4.01}$ \\
\hline S96T003163 & $146: 1$ & $\begin{array}{l}\text { Drainable } \\
\text { liquid }\end{array}$ & $<4 . \overline{01}$ & $<4.01$ & $<4.01$ \\
\hline
\end{tabular}


Table B2-28. Tank 241-U-108 Core Sample Analytical Results: Molybdenum (ICP).

\begin{tabular}{|c|c|c|c|c|c|}
\hline $\begin{array}{l}\text { Sumplo: } \\
\text { Nuniber }\end{array}$ & $\begin{array}{l}\text { Sample } \\
\text { I location }\end{array}$ & $\begin{array}{l}\text { Somple } \\
\text { Portinu }\end{array}$ & Resull: & Duplicate & Meas \\
\hline \multicolumn{2}{|c|}{ Solits a cir digest } & 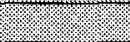 & (xg/g. & 408. & $10 \mathrm{~g}$ \\
\hline S96T003451 & Core 141 & $\begin{array}{l}\text { Solid } \\
\text { composite }\end{array}$ & 66.6 & 66 & 66.3 \\
\hline S96T003457 & Core 145 & $\begin{array}{l}\text { Solid } \\
\text { composite }\end{array}$ & $<49.5$ & $<48.4$ & $<48.95$ \\
\hline S96T004200 & Core 146 & $\begin{array}{l}\text { Solid } \\
\text { composite }\end{array}$ & 45.3 & 55.7 & $50.5^{\mathrm{QC}: \mathrm{e}}$ \\
\hline \multicolumn{2}{|c|}{. Sollds vater digest } & & 188 & $10 \mathrm{gg} 8$ & $1.2 .8 / 8:$ \\
\hline S96T003453 & Core 141 & $\begin{array}{l}\text { Solid } \\
\text { composite }\end{array}$ & 74 & 72.4 & 73.2 \\
\hline S96T003662 & Core 145 & $\begin{array}{l}\text { Solid } \\
\text { composite }\end{array}$ & 48.5 & 47.9 & 48.2 \\
\hline S96T004202 & Core 146 & $\begin{array}{l}\text { Solid } \\
\text { composite }\end{array}$ & 51.8 & 51.8 & 51.8 \\
\hline \multicolumn{2}{|c|}{ Ugaids } & 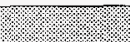 & $48 / \mathrm{m}$ & Hginl & 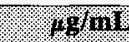 \\
\hline S96T002282 & $141: 1$ & $\begin{array}{l}\text { Drainable } \\
\text { liquid }\end{array}$ & 119 & 122 & 120.5 \\
\hline S96T002942 & $145: 1$ & $\begin{array}{l}\text { Drainable } \\
\text { liquid }\end{array}$ & 125 & 126 & 125.5 \\
\hline S96T003163 & 146: 1 & $\begin{array}{l}\text { Drainable } \\
\text { liquid }\end{array}$ & 125 & 119 & 122 \\
\hline
\end{tabular}


Table B2-29. Tank 241-U-108 Core Sample Analytical Results: Nickel (ICP). (3 sheets)

\begin{tabular}{|c|c|c|c|c|c|}
\hline $\begin{array}{l}\text { Gomingle } \\
\text { ramber }\end{array}$ & $\begin{array}{l}\text { Gampio } \\
\text { Eocation }\end{array}$ & $\begin{array}{l}\text { Sumplic } \\
\text { Portion. }\end{array}$ & Resilu & Buplicati & Mean \\
\hline \multicolumn{2}{|c|}{ Soldus geid bigest } & & s.ge & 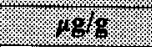 & $.148 \mathrm{~g}$ \\
\hline S96T003451 & Core 141 & $\begin{array}{l}\text { Solid } \\
\text { composite }\end{array}$ & 53.6 & 52.5 & 53.05 \\
\hline S96T003457 & Core 145 & $\begin{array}{l}\text { Solid } \\
\text { composite }\end{array}$ & 50.8 & 51.6 & 51.2 \\
\hline S96T004200 & Core 146 & $\begin{array}{l}\text { Solid } \\
\text { composite }\end{array}$ & 43.5 & 54.3 & $48.9^{\mathrm{QC}: \mathrm{e}}$ \\
\hline \multicolumn{2}{|c|}{ 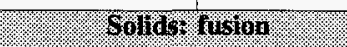 } & & 2.498 & 188 & 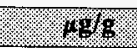 \\
\hline S96T002257 & 141: 1 & Lower $1 / 2$ & $<357$ & $<358$ & $<357.5$ \\
\hline S96T002258 & 141: 2 & Upper $1 / 2$ & $<372$ & $<387$ & $<379.5$ \\
\hline S96T002259 & $141: 3$ & Upper $1 / 2$ & $<351$ & $<345$ & $<348$ \\
\hline S96T002260 & 141: $3 A$ & Upper $1 / 2$ & $<391$ & $<376$ & $<383.5$ \\
\hline S96T002261 & \multirow[t]{2}{*}{ 141: 4} & Upper $1 / 2$ & $<365$ & $<363$ & $<364$ \\
\hline S96T002262 & & Lower $1 / 2$ & $<397$ & $<378$ & $<\longdiv { 3 8 7 . 5 }$ \\
\hline S96T002263 & \multirow[t]{2}{*}{ 141: $4 \mathrm{~A}$} & Upper $1 / 2$ & $<362$ & $<370$ & $<366$ \\
\hline S96T002264 & & Lower $1 / 2$ & $<361$ & $<357$ & $<359$ \\
\hline S96T002608 & \multirow[t]{2}{*}{$141: 5$} & Upper $1 / 2$ & $<377$ & $<382$ & $<379.5$ \\
\hline S96T002609 & & Lower $1 / 2$ & $<441$ & $<409$ & $<425$ \\
\hline S96T002610 & \multirow[t]{2}{*}{$141: 6$} & Upper 1/2 & $<424$ & $<385$ & $<404.5$ \\
\hline S96T002611 & & Lower $1 / 2$ & $<438$ & $<447$ & $<442.5$ \\
\hline S96T002612 & \multirow[t]{2}{*}{ 141: 7} & Upper $1 / 2$ & $<379$ & $<387$ & $<383$ \\
\hline S96T002613 & & Lower $1 / 2$ & $<441$ & $<424$ & $<432.5$ \\
\hline S96T002614 & \multirow[t]{2}{*}{$141: 8$} & Upper $1 / 2$ & $<411$ & $<373$ & $<392$ \\
\hline S96T002615 & & Lower $1 / 2$ & $<431$ & $<389$ & $<410^{\mathrm{QC}: \mathrm{e}}$ \\
\hline S96T002616 & $141: 9$ & Upper $1 / 2$ & $<432$ & $<423$ & $<427.5$ \\
\hline S96T002909 & $145: 2$ & Upper $1 / 2$ & $<393$ & $<388$ & $<390.5$ \\
\hline S96T002910 & \multirow[t]{2}{*}{$145: 3$} & Upper $1 / 2$ & $<404$ & $<404$ & $<404$ \\
\hline S96T002911 & & Lower $1 / 2$ & $<404$ & $<407$ & $<405.5$ \\
\hline S96T002912 & \multirow[t]{2}{*}{$145: 4$} & Upper $1 / 2$ & $<446$ & $<447$ & $<446.5$ \\
\hline S96T002913 & & Lower $1 / 2$ & $<422$ & $<436$ & $<429$ \\
\hline S96T002914 & \multirow[t]{2}{*}{$145: 5$} & Upper $1 / 2$ & $<442$ & $<438$ & $<440$ \\
\hline S96T002915 & & Lower $1 / 2$ & $<441$ & $<442$ & $<441.5$ \\
\hline S96T002916 & \multirow[t]{2}{*}{ 145: 6} & Upper $1 / 2$ & $<418$ & $<419$ & $<418.5$ \\
\hline S96T002917 & & Lower $1 / 2$ & $<401$ & $<396$ & $<398.5$ \\
\hline
\end{tabular}


Table B2-29. Tank 241-U-108 Core Sample Analytical Results: Nickel (ICP). (3 sheets)

\begin{tabular}{|c|c|c|c|c|c|}
\hline $\begin{array}{l}\text { Samples. } \\
\text { Numiger. }\end{array}$ & Simplo & Sample & Ressilt & byplicatc & yean \\
\hline \multicolumn{2}{|c|}{ 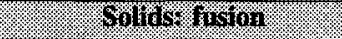 } & & $48 \mathrm{~g}$ & $4.9 \mathrm{~g} / \mathrm{g} \cdot \mathrm{s}$ & 1486 \\
\hline S96T002918 & \multirow[t]{2}{*}{$145: 7$} & Upper $1 / 2$ & $<414$ & $<412$ & $<413$ \\
\hline S96T002919 & & Lower $1 / 2$ & $<422$ & $<414$ & $<418$ \\
\hline S96T002920 & \multirow[t]{2}{*}{$145: 8$} & Upper $1 / 2$ & $<393$ & $<387$ & $<390$ \\
\hline S96T002921 & & Lower $1 / 2$ & $<409$ & $<410$ & $<\overline{409.5}$ \\
\hline S96T002922 & \multirow[t]{2}{*}{$145: 9$} & Upper $1 / 2$ & $<393$ & $<399$ & $<396$ \\
\hline S96T002923 & & Lower $1 / 2$ & $<393$ & $<393$ & $<393$ \\
\hline S96T003143 & 146: 1 & Upper $1 / 2$ & $<447$ & $<451$ & $<449$ \\
\hline S96T003144 & \multirow[t]{2}{*}{ 146: 2} & Upper $1 / 2$ & $<437$ & $<421$ & $<429$ \\
\hline S96T003145 & & Lower $1 / 2$ & $<373$ & $<454$ & $<413.5^{\mathrm{QC}: e}$ \\
\hline S96T003146 & \multirow[t]{2}{*}{ 146: 3} & Upper $1 / 2$ & $<444$ & $<451$ & $<447.5$ \\
\hline S96T003147 & & Lower $1 / 2$ & $<384$ & $<407$ & $<395.5$ \\
\hline S96T003001 & $146: 3 A$ & Upper $1 / 2$ & $<423$ & $<432$ & $<427.5$ \\
\hline S96T003002 & \multirow[t]{2}{*}{$146: 4$} & Upper $1 / 2$ & $<404$ & $<426$ & $<415$ \\
\hline S96T003003 & & Lower $1 / 2$ & $<390$ & $<425$ & $<407.5$ \\
\hline S96T003004 & \multirow[t]{2}{*}{ 146: 5} & Upper $1 / 2$ & $<438$ & $<388$ & $<413^{\mathrm{QC}: \mathrm{e}}$ \\
\hline S96T003005 & & Lower $1 / 2$ & $<418$ & $<396$ & $<407$ \\
\hline S96T003006 & \multirow[t]{2}{*}{$146: 6$} & Upper $1 / 2$ & $<452$ & $<382$ & $<417^{\mathrm{QC}: \mathrm{e}}$ \\
\hline S96T003007 & & Lower $1 / 2$ & $<408$ & $<413$ & $<410.5$ \\
\hline S96T003008 & \multirow[t]{2}{*}{$146: 7$} & Upper $1 / 2$ & $<391$ & $<407$ & $<399$ \\
\hline S96T003009 & & Lower $1 / 2$ & $<373$ & $<446$ & $<409.5^{\overline{\mathrm{QC}: \mathrm{e}}}$ \\
\hline S96T003148 & \multirow[t]{2}{*}{ 146: 8} & Upper $1 / 2$ & $<386$ & $<393$ & $<389.5$ \\
\hline S96T003149 & & Lower $1 / 2$ & $<393$ & $<378$ & $<385.5$ \\
\hline S96T004180 & \multirow[t]{2}{*}{ 146: 9} & Lower $1 / 2$ & $<395$ & $<398$ & $<396.5$ \\
\hline S96T003150 & & Upper $1 / 2$ & $<455$ & $<429$ & $<\overline{442}$ \\
\hline S96T003450 & Core 141 & $\begin{array}{l}\text { Solid } \\
\text { composite }\end{array}$ & $<395$ & $<404$ & $<399.5$ \\
\hline S96T003660 & Core 145 & $\begin{array}{l}\text { Solid } \\
\text { composite }\end{array}$ & $<395$ & $<312$ & $<353.5^{\mathrm{QC}: \mathrm{e}}$ \\
\hline S96T004199 & Core 146 & $\begin{array}{l}\text { Solid } \\
\text { composite }\end{array}$ & 2,280 & 1,920 & $2,100^{\mathrm{QC}: \mathrm{e}}$ \\
\hline
\end{tabular}


HNF-SD-WM-ER-639 Rev. 0

Table B2-29. Tank 241-U-108 Core Sample Analytical Results: Nickel (ICP). (3 sheets)

\begin{tabular}{|c|c|c|c|c|c|}
\hline Somple & Sample & $\begin{array}{l}\text { Saruplo } \\
\text { Portion }\end{array}$ & Result & Buplicate & Mean \\
\hline \multicolumn{2}{|c|}{ 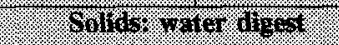 } & $9:=2$ & 4.68 & $1.48 \mathrm{~s}$. & $4 \mathrm{~g} / \mathrm{s}$ \\
\hline S96T003453 & Core 141 & $\begin{array}{l}\text { Solid } \\
\text { composite }\end{array}$ & $<20.5$ & $<20.5$ & $<20.5$ \\
\hline S96T003662 & Core 145 & $\begin{array}{l}\text { Solid } \\
\text { composite }\end{array}$ & $<18.8$ & $<14.6$ & $<\overline{16.7^{\mathrm{QC}: \mathrm{e}}}$ \\
\hline S96T004202 & Core 146 & $\begin{array}{l}\text { Solid } \\
\text { composite }\end{array}$ & 16.5 & 14.7 & $15.6^{\mathrm{QC}: e}$ \\
\hline \multicolumn{2}{|c|}{ Tiquidis: } & 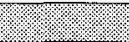 & $.14 \mathrm{~F} / \mathrm{mI}$ & 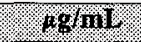 & T. $/ \mathrm{g} / \mathrm{mL}$ \\
\hline S96T002282 & 141: 1 & $\begin{array}{l}\text { Drainable } \\
\text { liquid }\end{array}$ & 27.3 & 30.3 & 28.8 \\
\hline S96T002942 & 145: 1 & $\begin{array}{l}\text { Drainable } \\
\text { liquid }\end{array}$ & 30.6 & 30.7 & 30.65 \\
\hline S96T003163 & 146: 1 & $\begin{array}{l}\text { Drainable } \\
\text { liquid }\end{array}$ & 35.2 & 33.4 & 34.3 \\
\hline
\end{tabular}


Table B2-30. Tank 241-U-108 Core Sample Analytical Results: Phosphorus (ICP). (3 sheets)

\begin{tabular}{|c|c|c|c|c|c|}
\hline $\begin{array}{l}\text { Somple } \\
\text { YHinuer }\end{array}$ & $\begin{array}{l}\text { Sample } \\
\text { yocrior }\end{array}$ & $\begin{array}{l}\text { Sample } \\
\text { Sortion. }\end{array}$ & Resint & Buplicare & Merar \\
\hline \multicolumn{2}{|c|}{ 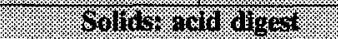 } & 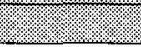 & 1.986 & 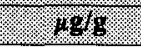 & 1.698 \\
\hline S96T003451 & Core 141 & $\begin{array}{l}\text { Solid } \\
\text { composite }\end{array}$ & 4,500 & 3,310 & $3,905^{\mathrm{QC}: \mathrm{e}}$ \\
\hline S96T003457 & Core 145 & $\begin{array}{l}\text { Solid } \\
\text { composite }\end{array}$ & 2,840 & 2,860 & 2,850 \\
\hline S96T004200 & Core 146 & $\begin{array}{l}\text { Solid } \\
\text { composite }\end{array}$ & 3,660 & $4, \overline{5} 10$ & $\overline{4,085^{\overline{Q C: c, e}}}$ \\
\hline \multicolumn{2}{|c|}{2 Sollas fusion } & W. & 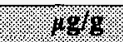 & $148 \%$ & 1048 \\
\hline S96T002257 & 141: 1 & Lower $1 / 2$ & 7,880 & 4,190 & $6,035^{\mathrm{QC:e}}$ \\
\hline S96T002258 & 141: 2 & Upper $1 / 2$ & $<3,720$ & $<3,870$ & $<3,795$ \\
\hline S96T002259 & 141: 3 & Upper $1 / 2$ & $<3,510$ & $<3,450$ & $<3,480$ \\
\hline S96T002260 & $141: 3 A$ & Upper $1 / 2$ & $<3,910$ & $<3,760$ & $<3,835$ \\
\hline S96T002261 & \multirow[t]{2}{*}{$141: 4$} & Upper $1 / 2$ & $<3,650$ & $<3,630$ & $<3,640$ \\
\hline S96T002262 & & Lower $1 / 2$ & $<3,970$ & $<3,780$ & $<\overline{3,875}$ \\
\hline S96T002263 & \multirow[t]{2}{*}{ 141: $4 \mathrm{~A}$} & Upper $1 / 2$ & $<3,620$ & $<3,700$ & $<3,660$ \\
\hline S96T002264 & & Lower $1 / 2$ & $<3,610$ & $<3,570$ & $<3,590$ \\
\hline S96T002608 & \multirow[t]{2}{*}{$141: 5$} & Upper $1 / 2$ & $<3,770$ & $<3,820$ & $<3,795$ \\
\hline S96T002609 & & Lower $1 / 2$ & $<4,410$ & $<4,090$ & $<4,250$ \\
\hline S96T002610 & \multirow[t]{2}{*}{ 141: 6} & Upper $1 / 2$ & $<4,240$ & $<3,850$ & $<4,045$ \\
\hline S96T002611 & & Lower $1 / 2$ & $<4,380$ & $<4,470$ & $<4,425$ \\
\hline S96T002612 & \multirow[t]{2}{*}{ 141: 7} & Upper $1 / 2$ & $<3,790$ & $<3,870$ & $<3,830$ \\
\hline S96T002613 & & Lower $1 / 2$ & $<4,410$ & $<4,240$ & $<4,325$ \\
\hline S96T002614 & \multirow[t]{2}{*}{$141: 8$} & Upper $1 / 2$ & $<4,110$ & $<3,730$ & $<3,920$ \\
\hline S96T002615 & & Lower $1 / 2$ & 11,200 & 11,000 & 11,100 \\
\hline S96T002616 & 141: 9 & Upper $1 / 2$ & 45,800 & 44,400 & 45,100 \\
\hline S96T002909 & 145: 2 & Upper $1 / 2$ & $<3,930$ & $<3,880$ & $<3,905$ \\
\hline S96T002910 & \multirow[t]{2}{*}{$145: 3$} & Upper $1 / 2$ & $<4,040$ & $<\overline{4,040}$ & $<4,040$ \\
\hline S96T002911 & & Lower $1 / 2$ & $<4,040$ & $<4,070$ & $<4,055$ \\
\hline S96T002912 & \multirow[t]{2}{*}{$145: 4$} & Upper $1 / 2$ & $<4,460$ & $<4,470$ & $<4,465$ \\
\hline S96T002913 & & Lower $1 / 2$ & $<4,220$ & $<4,360$ & $<4,290$ \\
\hline S96T002914 & \multirow[t]{2}{*}{$145: 5$} & Upper $1 / 2$ & $<4,420$ & $<4,380$ & $<4,400$ \\
\hline S96T002915 & & Lower $1 / 2$ & $<4,410$ & $<4,420$ & $<4,415$ \\
\hline S96T002916 & \multirow[t]{2}{*}{$145: 6$} & Upper $1 / 2$ & $<4,180$ & $<4,190$ & $<4,185$ \\
\hline S96T002917 & & Lower $1 / 2$ & $<4,010$ & $<3,960$ & $<3,985$ \\
\hline
\end{tabular}


Table B2-30. Tank 241-U-108 Core Sample Analytical Results: Phosphorus (ICP). ( 3 sheets)

\begin{tabular}{|c|c|c|c|c|c|}
\hline $\begin{array}{l}\text { Samples. } \\
\text { rinaliser. }\end{array}$ & $\begin{array}{l}\text { Sample. } \\
\text { locomion. }\end{array}$ & $\begin{array}{l}\text { Sample } \\
\text { rortion }\end{array}$ & Resiult & Buplicale? & Mean \\
\hline \multicolumn{2}{|c|}{ 1. Sollis Gurion } & 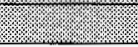 & 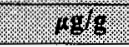 & 1.9918. & $.19 \mathrm{gb}$ \\
\hline S96T002918 & \multirow[t]{2}{*}{$145: 7$} & Upper $1 / 2$ & $<4,140$ & $<4,120$ & $<4,130$ \\
\hline S96T002919 & & Lower $1 / 2$ & $<4,220$ & $<4,140$ & $<4,180$ \\
\hline S96T002920 & \multirow[t]{2}{*}{$145: 8$} & Upper $1 / 2$ & $<3,930$ & $<3,870$ & $<3,900$ \\
\hline S96T002921 & & Lower $1 / 2$ & $<4,090$ & $<4,100$ & $<4,095$ \\
\hline S96T002922 & \multirow[t]{2}{*}{$145: 9$} & Upper $1 / 2$ & 15,400 & 20,000 & $17,700^{\mathrm{eC}: \mathrm{e}}$ \\
\hline S96T002923 & & Lower $1 / 2$ & 47,000 & 48,100 & 47,550 \\
\hline S96T003143 & 146: 1 & Upper $1 / 2$ & $<4,470$ & $<4,510$ & $<4,490$ \\
\hline S96T003144 & \multirow[t]{2}{*}{$146: 2$} & Upper $1 / 2$ & $<4,370$ & $<4,210$ & $<4,290$ \\
\hline S96T003145 & & Lower $1 / 2$ & $<3,730$ & $<4,540$ & $<4,135^{\mathrm{QC:e}}$ \\
\hline S96T003146 & \multirow[t]{2}{*}{$146: 3$} & Upper $1 / 2$ & $<4,440$ & $<4,510$ & $<4,475$ \\
\hline S96T003147 & & Lower $1 / 2$ & $<3,840$ & $<4,070$ & $<3, \overline{955}$ \\
\hline S96T003001 & 146: $3 \mathrm{~A}$ & Upper $1 / 2$ & $<4,230$ & $<4,320$ & $<4,275$ \\
\hline S96T003002 & \multirow[t]{2}{*}{$146: 4$} & Upper $1 / 2$ & $<4,040$ & $<4,260$ & $<4,150$ \\
\hline S96T003003 & & Lower $1 / 2$ & $<3,900$ & $<4,250$ & $<4,075$ \\
\hline S96T003004 & \multirow[t]{2}{*}{$146: 5$} & Upper $1 / 2$ & $<4,380$ & $<3,880$ & $<4,130^{\text {QC:e }}$ \\
\hline S96T003005 & & Lower $1 / 2$ & $<4,180$ & $<3,960$ & $<4,070$ \\
\hline S96T003006 & \multirow[t]{2}{*}{$146: 6$} & Upper $1 / 2$ & $<4,520$ & $<3,820$ & $<4,170^{\mathrm{QC: \textrm {e }}}$ \\
\hline S96T003007 & & Lower $1 / 2$ & $<4,080$ & $<4,130$ & $<4,105$ \\
\hline S96T003008 & \multirow[t]{2}{*}{ 146: 7} & Upper $1 / 2$ & $<3,910$ & $<4,070$ & $<3,990$ \\
\hline S96T003009 & & Lower $1 / 2$ & $<3,730$ & $<4,460$ & $<4,095^{\mathrm{Qc}: \mathrm{e}}$ \\
\hline S96T003148 & \multirow[t]{2}{*}{$146: 8$} & Upper $1 / 2$ & $<3,860$ & $<3,930$ & $<3,895$ \\
\hline S96T003149 & & Lower $1 / 2$ & $<3,930$ & $<3,780$ & $<3,855$ \\
\hline S96T004180 & \multirow[t]{2}{*}{$146: 9$} & Lower $1 / 2$ & 43,300 & 46,800 & 45,050 \\
\hline S96T003150 & & Upper $1 / 2$ & $<4,550$ & 4,690 & $<4,620$ \\
\hline S96T003450 & Core 141 & $\begin{array}{l}\text { Solid } \\
\text { composite }\end{array}$ & $<3,950$ & $<4,040$ & $<3,995$ \\
\hline S96T003660 & Core 145 & \begin{tabular}{|l|}
$\begin{array}{l}\text { Solid } \\
\text { composite }\end{array}$ \\
\end{tabular} & $<3,950$ & $<3,120$ & $<3,535^{\mathrm{QC} e \mathrm{e}}$ \\
\hline S96T004199 & Core 146 & $\begin{array}{l}\text { Solid } \\
\text { composite }\end{array}$ & 4,090 & $<3,930$ & $<4,010$ \\
\hline
\end{tabular}


Table B2-30. Tank 241-U-108 Core Sample Analytical Results: Phosphorus (ICP). ( 3 sheets)

\begin{tabular}{|c|c|c|c|c|c|}
\hline Sample & $\begin{array}{l}\text { Sannple } \\
\text { Yocation }\end{array}$ & $\begin{array}{l}\text { Sample } \\
\text { Iiontion }\end{array}$ & 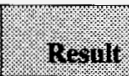 & Binileate & Mean \\
\hline \multicolumn{2}{|c|}{ Sollats vater aiget } & & 16918 & 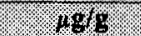 & $48 \%$ \\
\hline S96T003453 & Core 141 & $\begin{array}{l}\text { Solid } \\
\text { composite }\end{array}$ & 4,380 & 5,190 & $4,785^{\mathrm{QC}: \mathrm{e}}$ \\
\hline S96T003662 & Core 145 & $\begin{array}{l}\text { Solid } \\
\text { composite }\end{array}$ & 3,290 & $3, \overline{020}$ & 3,155 \\
\hline S96T004202 & Core 146 & $\begin{array}{l}\text { Solid } \\
\text { composite }\end{array}$ & 4,040 & 4,050 & 4,045 \\
\hline \multicolumn{2}{|c|}{ Lquares: } & & $1.8 \mathrm{ginu}$ & 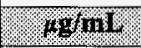 & $4.9 \mathrm{gmi}$ \\
\hline S96T002282 & $141: 1$ & $\begin{array}{l}\text { Drainable } \\
\text { liquid }\end{array}$ & 967 & 957 & 962 \\
\hline S96T002942 & 145: 1 & $\begin{array}{l}\text { Drainable } \\
\text { liquid }\end{array}$ & 1,030 & 1,040 & 1,035 \\
\hline S96T003163 & 146: 1 & $\begin{array}{l}\text { Drainable } \\
\text { liquid }\end{array}$ & 1,070 & 1,030 & 1,050 \\
\hline
\end{tabular}


Table B2-31. Tank 241-U-108 Core Sample Analytical Results: Potassium (ICP).

\begin{tabular}{|c|c|c|c|c|c|}
\hline S4ming & S Sample & $\begin{array}{l}\text { Sample } \\
\text { Portion }\end{array}$ & Iesuil & Biplicalo & Mean \\
\hline \multicolumn{2}{|c|}{ Solits acid digest } & & 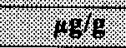 & $.19 \mathrm{~g} / \mathrm{s}$ & . $286 \mathrm{~g}$ \\
\hline S96T003451 & Core 141 & $\begin{array}{l}\text { Solid } \\
\text { composite }\end{array}$ & 1,980 & 1,830 & 1,905 \\
\hline S96T003457 & Core 145 & $\begin{array}{l}\text { Solid } \\
\text { composite }\end{array}$ & 1,300 & 1,220 & 1,260 \\
\hline S96T004200 & Core 146 & $\begin{array}{l}\text { Solid } \\
\text { composite }\end{array}$ & 1,470 & 1,680 & $1,575^{\mathrm{QC}: \mathrm{c}, \mathrm{e}}$ \\
\hline \multicolumn{2}{|c|}{ Solliks trater digest } & ?:- & 1098 & 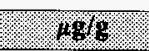 & .8969 \\
\hline S96T003453 & Core 141 & $\begin{array}{l}\text { Solid } \\
\text { composite }\end{array}$ & 2,050 & 2,070 & 2,060 \\
\hline S96T003662 & Core 145 & $\begin{array}{l}\text { Solid } \\
\text { composite }\end{array}$ & 1,420 & 1,430 & 1,425 \\
\hline S96T004202 & Core 146 & $\begin{array}{l}\text { Solid } \\
\text { composite }\end{array}$ & 1,520 & 1,530 & $1,5 \overline{25}$ \\
\hline \multicolumn{2}{|c|}{. Liquids: } & (2.1. & $.89 \mathrm{mil}$ & $.9 \mathrm{~g} \mathrm{~mL}$ & $10 \mathrm{gr} \mathbf{m L}$ \\
\hline S96T002282 & 141: 1 & $\begin{array}{l}\text { Drainable } \\
\text { liquid }\end{array}$ & 3,900 & 4,040 & 3,970 \\
\hline S96T002942 & 145: 1 & $\begin{array}{l}\text { Drainable } \\
\text { liquid }\end{array}$ & $4, \overline{160}$ & 4,120 & 4,140 \\
\hline S96T003163 & 146: 1 & $\begin{array}{l}\text { Drainable } \\
\text { liquid }\end{array}$ & 3,970 & 3,870 & 3,920 \\
\hline
\end{tabular}


Table B2-32. Tank 241-U-108 Core Sample Analytical Results: Silicon (ICP). (3 sheets)

\begin{tabular}{|c|c|c|c|c|c|}
\hline $\begin{array}{l}\text { Samples } \\
\text { Numiler. }\end{array}$ & $\begin{array}{l}\text { Gample } \\
\text { Lagation }\end{array}$ & $\begin{array}{l}\text { Sample: } \\
\text { Portion }\end{array}$ & Iescuit) & Buplicare: & Vean \\
\hline \multicolumn{2}{|c|}{ 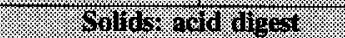 } & & $148 / \mathrm{s}$ & $4 \mathrm{glg}$ & 1089 \\
\hline S96T003451 & Core 141 & $\begin{array}{l}\text { Solid } \\
\text { composite }\end{array}$ & 147 & 161 & 154 \\
\hline S96T003457 & Core 145 & $\begin{array}{l}\text { Solid } \\
\text { composite }\end{array}$ & 108 & 92.6 & $100.3^{\mathrm{QC}: \mathrm{e}}$ \\
\hline S96T004200 & Core 146 & $\begin{array}{l}\text { Solid } \\
\text { composite }\end{array}$ & 95.3 & 110 & $102.7^{\mathrm{QC}: \mathrm{b}, \mathrm{e}}$ \\
\hline \multicolumn{2}{|c|}{ Soltds: hision } & 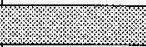 & 488 & 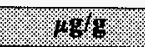 & 2. $\mathrm{pg} / \mathrm{g}$ \\
\hline S96T002257 & 141: 1 & Lower $1 / 2$ & $<893$ & $<895$ & $<894$ \\
\hline S96T002258 & 141: 2 & Upper $1 / 2$ & $<929$ & $<967$ & $<948$ \\
\hline S96T002259 & $141: 3$ & Upper $1 / 2$ & $<878$ & $<864$ & $<\overline{871}$ \\
\hline S96T002260 & 141: $3 \bar{A}$ & Upper $1 / 2$ & $<978$ & 1,180 & $<1,080^{\mathrm{QCe} e}$ \\
\hline S96T002261 & \multirow[t]{2}{*}{$141: 4$} & Upper $1 / 2$ & $<913$ & $<907$ & $<99$ \\
\hline S96T002262 & & Lower $1 / 2$ & $<991$ & $<945$ & $<968$ \\
\hline S96T002263 & \multirow[t]{2}{*}{ 141: $4 \mathrm{~A}$} & Upper $1 / 2$ & $<904$ & $<925$ & $<914.5$ \\
\hline S96T002264 & & Lower $1 / 2$ & $<904$ & 932 & $<918$ \\
\hline S96T002608 & \multirow[t]{2}{*}{ 141: 5} & Upper $1 / 2$ & $<944$ & $<954$ & $<949$ \\
\hline S96T002609 & & Lower $1 / 2$ & $<1,100$ & 1,590 & $<1,340^{\mathrm{oC}: \mathrm{e}}$ \\
\hline S96T002610 & \multirow[t]{2}{*}{ 141: 6} & Upper $1 / 2$ & $<1,060$ & $<\overline{963}$ & $<1,011$ \\
\hline S96T002611 & & Lower $1 / 2$ & $<1,100$ & $<1,120$ & $<1,110$ \\
\hline S96T002612 & \multirow[t]{2}{*}{ 141: 7} & Upper $1 / 2$ & $<947$ & $<968$ & $<957.5$ \\
\hline S96T002613 & & Lower $1 / 2$ & 2,450 & $<1,060$ & $<1,755^{\mathrm{QC}: \mathrm{e}}$ \\
\hline S96T002614 & \multirow[t]{2}{*}{$141: 8$} & Upper $1 / 2$ & $<1,030$ & $<934$ & $<982$ \\
\hline S96T002615 & & Lower $1 / 2$ & $<1,080$ & $<973$ & $<1,026^{\mathrm{QC:e}}$ \\
\hline S96T002616 & 141: 9 & Upper $1 / 2$ & 1,930 & 2,390 & $2,160^{\mathrm{Q} C: \mathrm{e}}$ \\
\hline S96T002909 & $145: 2$ & Upper $1 / 2$ & 984 & $<970$ & $<977$ \\
\hline S96T002910 & \multirow[t]{2}{*}{$145: 3$} & Upper $1 / 2$ & $<1,010$ & $<1,010$ & $<1,010$ \\
\hline S96T002911 & & Lower $1 / 2$ & $<1,010$ & $<1,020$ & $<1,015$ \\
\hline S96T002912 & \multirow[t]{2}{*}{$145: 4$} & Upper $1 / 2$ & $<1,110$ & $<1,120$ & $<1,115$ \\
\hline S96T002913 & & Lower $1 / 2$ & $<1,050$ & $<1,090$ & $<1,070$ \\
\hline S96T002914 & \multirow[t]{2}{*}{$145: 5$} & Upper $1 / 2$ & $<1,110$ & $<1,090$ & $<1,100$ \\
\hline S96T002915 & & Lower $1 / 2$ & $<1,100$ & $<1,110$ & $<1,105$ \\
\hline S96T002916 & \multirow[t]{2}{*}{$145: 6$} & Upper $1 / 2$ & $<1,040$ & $<1,050$ & $<1,045$ \\
\hline S96T002917 & & Lower $1 / 2$ & $<1,000$ & $<990$ & $<995$ \\
\hline
\end{tabular}


Table B2-32. Tank 241-U-108 Core Sample Analytical Results: Silicon (ICP). (3 sheets)

\begin{tabular}{|c|c|c|c|c|c|}
\hline Sarmple: & Somple & $\begin{array}{l}\text { Sample } \\
\text { Porioror }\end{array}$ & Result & Mapilozre & Hean \\
\hline \multicolumn{2}{|c|}{ S6hios: Gikinar: } & 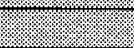 & 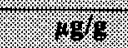 & 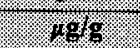 & $18 \mathrm{gg}$ \\
\hline S96T002918 & \multirow[t]{2}{*}{$145: 7$} & Upper 1/2 & $<1,040$ & $<1,030$ & $<1,035$ \\
\hline S96T002919 & & Lower $1 / 2$ & $<1,050$ & $<1,040$ & $<1,045$ \\
\hline S96T002920 & \multirow[t]{2}{*}{$145: 8$} & Upper $1 / 2$ & $<983$ & $<967$ & $<975$ \\
\hline S96T002921 & & Lower $1 / 2$ & $<\overline{1,020}$ & $<1,020$ & $<.1,020$ \\
\hline S96T002922 & \multirow[t]{2}{*}{$145: 9$} & Upper $1 / 2$ & $<982$ & $<998$ & $<990$ \\
\hline S96T002923 & & Lower $1 / 2$ & $<982$ & $<984$ & $<983$ \\
\hline S96T003143 & 146: 1 & Upper $1 / 2$ & $<1,120$ & $<1,130$ & $<1,125$ \\
\hline S96T003144 & \multirow[t]{2}{*}{ 146: 2} & Upper $1 / 2$ & $<1,090$ & $<1,050$ & $<1,070$ \\
\hline S96T003145 & & Lower $1 / 2$ & $<934$ & $<1,130$ & $<1,032^{\mathrm{QC}: \mathrm{e}}$ \\
\hline S96T003146 & \multirow[t]{2}{*}{ 146: 3} & Upper $1 / 2$ & $<1,110$ & $<1,130$ & $<1,120$ \\
\hline S96T003147 & & Lower $1 / 2$ & $<961$ & $<1,020$ & $<990.5$ \\
\hline S96T003001 & 146: $3 A$ & Upper $1 / 2$ & $<1,060$ & $<1,080$ & $<1,070$ \\
\hline S96T003002 & \multirow[t]{2}{*}{$146: 4$} & Upper $1 / 2$ & $<1,010$ & $<1,060$ & $<1,035$ \\
\hline S96T003003 & & Lower $1 / 2$ & $<976$ & $<1,060$ & $<1,018$ \\
\hline S96T003004 & \multirow[t]{2}{*}{$146: 5$} & Upper $1 / 2$ & $<1,100$ & $<969$ & $<1,034^{\mathrm{QC}: \mathrm{e}}$ \\
\hline S96T003005 & & Lower $1 / 2$ & $<1,050$ & $<990$ & $<1,020$ \\
\hline S96T003006 & \multirow[t]{2}{*}{ 146: 6} & Upper $1 / 2$ & $<1,130$ & $<954$ & $<1,042^{\mathrm{QC}: \mathrm{e}}$ \\
\hline S96T003007 & & Lower $1 / 2$ & $<1,020$ & $<1,030$ & $<1,025$ \\
\hline S96T003008 & \multirow[t]{2}{*}{ 146: 7} & Upper $1 / 2$ & $<978$ & $<1,020$ & $<\overline{999}$ \\
\hline S96T003009 & & Lower $1 / 2$ & $<932$ & $<1,110$ & $<1,021^{\mathrm{QC}: \mathrm{e}}$ \\
\hline S96T003148 & \multirow[t]{2}{*}{ 146: 8} & Upper $1 / 2$ & 2,980 & $<982$ & $<1,981^{\mathrm{QC}: \mathrm{e}}$ \\
\hline S96T003149 & & Lower $1 / 2$ & $<982$ & $<946$ & $<964$ \\
\hline S96T004 180 & \multirow[t]{2}{*}{ 146: 9} & Lower $1 / 2$ & $<987$ & $<994$ & $<990.5$ \\
\hline S96T003150 & & Upper $1 / 2$ & $<1,140$ & $<1,070$ & $<1,105$ \\
\hline S96T003450 & Core 141 & $\begin{array}{l}\text { Solid } \\
\text { composite }\end{array}$ & $<987$ & $<1,010$ & $<998.5$ \\
\hline S96T003660 & Core 145 & $\begin{array}{l}\text { Solid } \\
\text { composite }\end{array}$ & $<987$ & $<781$ & $<884^{\mathrm{QC}: \mathrm{e}}$ \\
\hline S96T004199 & Core 146 & $\begin{array}{l}\text { Solid } \\
\text { composite }\end{array}$ & $<1,010$ & $<981$ & $<995.5$ \\
\hline
\end{tabular}


Table B2-32. Tank 241-U-108 Core Sample Analytical Results: Silicon (ICP). (3 sheets)

\begin{tabular}{|c|c|c|c|c|c|}
\hline $\begin{array}{l}\text { sample. } \\
\text { fymlor }\end{array}$ & samigle & $\begin{array}{l}\text { Sample } \\
\text { Portion }\end{array}$ & Resull & Buplicate & Mean \\
\hline \multicolumn{2}{|c|}{ Gollos Yriter digest } & 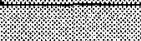 & $14 \mathrm{~g} / \mathrm{P}$ & . 159 & iste: \\
\hline S96T003453 & Core 141 & $\begin{array}{l}\text { Solid } \\
\text { composite }\end{array}$ & 308 & 351 & $329.5^{\mathrm{QC:e}}$ \\
\hline S96T003662 & Core 145 & $\begin{array}{l}\text { Solid } \\
\text { composite }\end{array}$ & 271 & 202 & $236.5^{Q C ; e}$ \\
\hline S96T004202 & Core 146 & $\begin{array}{l}\text { Solid } \\
\text { composite }\end{array}$ & 304 & 259 & $281.5^{\mathrm{QC}: \mathrm{e}}$ \\
\hline \multicolumn{2}{|c|}{. Yquils } & : & folinl & $1 \mathrm{~g} / \mathrm{mL}$ & $1 \% \mathrm{~g} / \mathrm{mI}$ \\
\hline S96T002282 & 141: 1 & $\begin{array}{l}\text { Drainable } \\
\text { liquid }\end{array}$ & 201 & 205 & 203 \\
\hline S96T002942 & 145: 1 & $\begin{array}{l}\text { Drainable } \\
\text { liquid }\end{array}$ & 185 & 186 & 185.5 \\
\hline S96T003163 & 146: 1 & $\begin{array}{l}\text { Drainable } \\
\text { liquid }\end{array}$ & 202 & 190 & 196 \\
\hline
\end{tabular}


Table B2-33. Tank 241-U-108 Core Sample Analytical Results: Silver (ICP).

\begin{tabular}{|c|c|c|c|c|c|}
\hline Shoriof & W. & 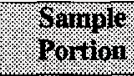 & $\mathrm{Resilt}$ & Myplicate & Mean \\
\hline \multicolumn{2}{|c|}{. } & & 489 & 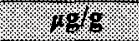 & .4 .486 \\
\hline S96T003451 & Core 141 & $\begin{array}{l}\text { Solid } \\
\text { composite }\end{array}$ & $<9.6$ & $<9.63$ & $<9.615^{\mathrm{QC}: \mathrm{s}}$ \\
\hline S96T003457 & Core 145 & $\begin{array}{l}\text { Solid } \\
\text { composite }\end{array}$ & $<9.89$ & $<9.67$ & $<9.78^{\mathrm{QC:a}}$ \\
\hline S96T004200 & Core 146 & $\begin{array}{l}\text { Solid } \\
\text { composite }\end{array}$ & 15.1 & 17.3 & $16.2^{\mathrm{QCa}, \mathrm{e}}$ \\
\hline \multicolumn{2}{|c|}{ Sollow ratter digert } & & $19 \% 8$ & 1898 & 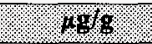 \\
\hline S96T003453 & Core 141 & $\begin{array}{l}\text { Solid } \\
\text { composite }\end{array}$ & $<10.3$ & $<10.2$ & $<10.25$ \\
\hline S96T003662 & Core 145 & $\begin{array}{l}\text { Solid } \\
\text { composite }\end{array}$ & $<9.42$ & $<7.28$ & $<8.35^{\mathrm{QC:}: \mathrm{e}}$ \\
\hline S96T004202 & Core 146 & $\begin{array}{l}\text { Solid } \\
\text { composite }\end{array}$ & 15.7 & 15.2 & 15.45 \\
\hline \multicolumn{2}{|c|}{ 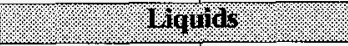 } & & 189 , & $189 \mathrm{~min}$ & $.0 .8101 \%$ \\
\hline S96T002282 & 141: 1 & $\begin{array}{l}\text { Drainable } \\
\text { liquid }\end{array}$ & 15.8 & 15.8 & 15.8 \\
\hline S96T002942 & $145: 1$ & $\begin{array}{l}\text { Drainable } \\
\text { liquid }\end{array}$ & 17.3 & 16.8 & 17.05 \\
\hline \$96T003163 & $146: 1$ & $\begin{array}{l}\text { Drainable } \\
\text { liquid }\end{array}$ & 16.2 & 15.9 & 16.05 \\
\hline
\end{tabular}


Table B2-34. Tank 241-U-108 Core Sample Analytical Results: Sodium (ICP), (3 sheets)

\begin{tabular}{|c|c|c|c|c|c|}
\hline $\begin{array}{l}\text { Shingle } \\
\text { romolice }\end{array}$ & $\begin{array}{l}\text { Simple } \\
\text { I geafion }\end{array}$ & $\begin{array}{l}\text { Sample } \\
\text { Sorrtion }\end{array}$ & Reswit & Diplicare & Mean: \\
\hline \multicolumn{2}{|c|}{ Soltis adod abert : } & & .096 & 4.919 & (.). 194 \\
\hline S96T003451 & Core 141 & $\begin{array}{l}\text { Solid } \\
\text { composite }\end{array}$ & $1.930 \mathrm{E}+05$ & $1.900 \mathrm{E}+05$ & ${ }_{Q \dot{c}: b, d} 1,5 \mathrm{E}+05$ \\
\hline S96T003457 & Core 145 & $\begin{array}{l}\text { Solid } \\
\text { composite }\end{array}$ & $2.080 \mathrm{E}+05$ & $2.180 \mathrm{E}+05$ & $2.130 \overline{\mathrm{E}}+05^{\mathrm{QC}: \mathrm{b}}$ \\
\hline S96T004200 & Core 146 & $\begin{array}{l}\text { Solid } \\
\text { composite }\end{array}$ & $1.930 \mathrm{E}+05$ & $2.320 \mathrm{E}+05$ & $2.125 \overline{\mathrm{E}+05^{\mathrm{QC}: \mathrm{b}, \mathrm{c}}}$ \\
\hline \multicolumn{2}{|c|}{ Solids rusion } & & 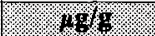 & 4818 & $1.9 \%$ \\
\hline S96T002257 & $141: 1$ & Lower $1 / 2$ & $1.850 \mathrm{E}+05$ & $1.800 \mathrm{E}+05$ & $1.825 \mathrm{E}+05$ \\
\hline S96T002258 & $141: 2$ & Upper $1 / 2$ & $2.220 \mathrm{E}+05$ & $2.220 \mathrm{E}+05$ & $2.220 \mathrm{E}+05$ \\
\hline S96T002259 & $141: 3$ & Upper $1 / 2$ & $2.240 \mathrm{E}+05$ & $2.210 \bar{E}+05$ & $2.225 \mathrm{E}+05$ \\
\hline S96T002260 & $141: 3 A$ & Upper $1 / 2$ & $2.250 \overline{\mathrm{E}}+05$ & $2.250 \mathrm{E}+05$ & $2 . \overline{250} \mathrm{E}+05$ \\
\hline S96T002261 & \multirow[t]{2}{*}{$141: 4$} & Upper $1 / 2$ & $2.150 \mathrm{E}+05$ & $2.080 \mathrm{E}+05$ & $2.115 \mathrm{E}+05$ \\
\hline S96T002262 & & Lower $1 / 2$ & $2.150 \mathrm{E}+05$ & $2.090 \mathrm{E}+05$ & $2.120 \mathrm{E}+05$ \\
\hline S96T002263 & \multirow[t]{2}{*}{ 141: $4 \mathrm{~A}$} & Upper $1 / 2$ & $2.180 \mathrm{E}+05$ & $2.200 \mathrm{E}+05$ & $2.190 \mathrm{E}+05$ \\
\hline S96T002264 & & Lower $1 / 2$ & $2.130 \mathrm{E}+05$ & $2.160 \mathrm{E}+05$ & $2.145 E+05$ \\
\hline S96T002608 & \multirow[t]{2}{*}{$141: 5$} & Upper $1 / 2$ & $2.610 \mathrm{E}+05$ & $2.690 \mathrm{E}+05$ & $2.650 \overline{\mathrm{E}}+05$ \\
\hline S96T002609 & & Lower $1 / 2$ & $2.830 \mathrm{E}+05$ & $2.760 \mathrm{E}+05$ & $2.795 \mathrm{E}+05$ \\
\hline \$96T002610 & \multirow[t]{2}{*}{$141: 6$} & Upper $1 / 2$ & $2.860 \mathrm{E}+05$ & $2.710 \mathrm{E}+05$ & $2.785 \mathrm{E}+05$ \\
\hline S96T002611 & & Lower $1 / 2$ & $2.850 \mathrm{E}+05$ & $2.770 \mathrm{E}+05$ & $2.810 \overline{\mathrm{E}+05}$ \\
\hline S96T002612 & \multirow[t]{2}{*}{ 141: 7} & Upper $1 / 2$ & $2.550 \mathrm{E}+05$ & $2.480 \mathrm{E}+05$ & $2.515 \mathrm{E}+05$ \\
\hline S96T002613 & & Lower $1 / 2$ & $2.650 \mathrm{E}+05$ & $2.610 \mathrm{E}+05$ & $2.630 \mathrm{E}+05$ \\
\hline S96T002614 & \multirow[t]{2}{*}{ 141: 8} & Upper $1 / 2$ & $2.400 \mathrm{E}+05$ & $2.470 \mathrm{E}+05$ & $2.435 \mathrm{E}+05$ \\
\hline S96T002615 & & Lower $1 / 2$ & $2.630 \overline{\mathrm{E}}+05$ & $2.530 \mathrm{E}+05$ & $2.580 \mathrm{E}+05$ \\
\hline S96T002616 & 141: 9 & Upper $1 / 2$ & $1.900 \mathrm{E}+05$ & $1.960 \overline{\mathrm{E}}+05$ & $1.930 \mathrm{E}+05$ \\
\hline S96T002909 & $145: 2$ & Upper $1 / 2$ & $2.570 \mathrm{E}+05$ & $2.570 \overline{\mathrm{E}+05}$ & $2.570 \mathrm{E}+05$ \\
\hline S96T002910 & \multirow[t]{2}{*}{$145: 3$} & Upper $1 / 2$ & $2.590 \mathrm{E}+05$ & $2.450 \mathrm{E}+05$ & $2.520 \mathrm{E}+05$ \\
\hline S96T002911 & & Lower $1 / 2$ & $2.690 \mathrm{E}+05$ & $2.670 \mathrm{E}+05$ & $2.680 \mathrm{E}+05$ \\
\hline S96T002912 & \multirow[t]{2}{*}{$145: 4$} & Upper $1 / 2$ & $2.740 \mathrm{E}+05$ & $2.680 \mathrm{E}+05$ & $2.710 \mathrm{E}+05$ \\
\hline S96T002913 & & Lower $1 / 2$ & $2.770 \mathrm{E}+05$ & $2.820 \mathrm{E}+05$ & $2.795 \mathrm{E}+05$ \\
\hline S96T002914 & \multirow[t]{2}{*}{$145: 5$} & Upper $1 / 2$ & $2.880 \mathrm{E}+05$ & $2.790 \mathrm{E}+05$ & $2.835 \mathrm{E}+05$ \\
\hline S96T002915 & & Lower $1 / 2$ & $2.630 \mathrm{E}+05$ & $2.580 \mathrm{E}+05$ & $2.605 \mathrm{E}+05$ \\
\hline S96T002916 & \multirow[t]{2}{*}{$145: 6$} & Upper $1 / 2$ & $2.700 \mathrm{E}+05$ & $2.660 \mathrm{E}+05$ & $2.680 \bar{E}+05$ \\
\hline S96T002917 & & Lower $1 / 2$ & $2.670 \mathrm{E}+05$ & $2.710 \mathrm{E}+05$ & $2.690 \mathrm{E}+05$ \\
\hline
\end{tabular}


Table B2-34. Tank 241-U-108 Core Sample Analytical Results: Sodium (ICP), (3 sheets)

\begin{tabular}{|c|c|c|c|c|c|}
\hline $\begin{array}{l}\text { Samples } \\
\text { Number. }\end{array}$ & 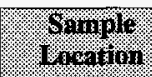 & $\begin{array}{l}\text { Sinnple: } \\
\text { Gortion: }\end{array}$ & Retril & Buplicare & Mean \\
\hline \multicolumn{2}{|c|}{ Soling liston } & & 498 & 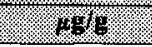 & 1.1940 \\
\hline S96T002918 & \multirow[t]{2}{*}{$145: 7$} & Upper $1 / 2$ & $2.610 \mathrm{E}+05$ & $2.590 \mathrm{E}+05$ & $2.600 \mathrm{E}+05$ \\
\hline S96T002919 & & Lower $1 / 2$ & $2.170 \mathrm{E}+05$ & $2.120 \mathrm{E}+05$ & $2.145 \mathrm{E}+05$ \\
\hline S96T002920 & \multirow[t]{2}{*}{ 145: 8} & Upper $1 / 2$ & $2.370 \mathrm{E}+05$ & $2.320 \mathrm{E}+05$ & $2.345 \mathrm{E}+05$ \\
\hline S96T002921 & & Lower $1 / 2$ & $2.460 \mathrm{E}+05$ & $2.280 \mathrm{E}+05$ & $2.370 \mathrm{E}+05$ \\
\hline S96T002922 & \multirow[t]{2}{*}{$145: 9$} & Upper $1 / 2$ & $2.090 \mathrm{E}+05$ & $2.1 \overline{10} \mathrm{E}+05$ & $2.100 \bar{E}+05$ \\
\hline S96T002923 & & Lower $1 / 2$ & $1.810 \mathrm{E}+05$ & $1.830 \mathrm{E}+05$ & $1.820 \bar{E}+05$ \\
\hline S96T003143 & 146: 1 & Upper $1 / 2$ & $2.220 \mathrm{E}+05$ & $2.200 \mathrm{E}+05$ & $2.210 \overline{\mathrm{E}}+05$ \\
\hline S96T003144 & \multirow[t]{2}{*}{$146: 2$} & Upper $1 / 2$ & $2.340 \mathrm{E}+05$ & $2.360 \mathrm{E}+05$ & $2.350 \mathrm{E}+05$ \\
\hline S96T003145 & & Lower $1 / 2$ & $2.390 \mathrm{E}+05$ & $2.360 \overline{\mathrm{E}+05}$ & $2.375 \mathrm{E}+05$ \\
\hline S96T003146 & \multirow[t]{2}{*}{ 146: 3} & Upper $1 / 2$ & $2.290 \mathrm{E}+05$ & $2.360 \mathrm{E}+05$ & $2.325 \bar{E}+05$ \\
\hline S96T003147 & & Lower $1 / 2$ & $2.340 \overline{\mathrm{E}+05}$ & $2.330 \mathrm{E}+05$ & $2.335 \mathrm{E}+05$ \\
\hline S96T003001 & 146: $3 \mathrm{~A}$ & Upper $1 / 2$ & $2.330 \mathrm{E}+05$ & $2.330 \mathrm{E}+05$ & $2.330 \overline{\mathrm{E}+05}$ \\
\hline S96T003002 & \multirow[t]{2}{*}{$146: 4$} & Upper $1 / 2$ & $3.040 \mathrm{E}+05$ & $3.030 \mathrm{E}+05$ & $3.035 \mathrm{E}+05$ \\
\hline S96T003003 & & Lower $1 / 2$ & $2.890 \mathrm{E}+05$ & $2.930 \mathrm{E}+05$ & $2.910 \mathrm{E}+05$ \\
\hline S96T003004 & \multirow[t]{2}{*}{$146: 5$} & Upper $1 / 2$ & $2.930 \mathrm{E}+05$ & $2.660 \mathrm{E}+05$ & $2.795 \mathrm{E}+05$ \\
\hline S96T003005 & & Lower $1 / 2$ & $2.320 \mathrm{E}+05$ & $2.270 \mathrm{E}+\overline{05}$ & $2.295 \mathrm{E}+05$ \\
\hline S96T003006 & \multirow[t]{2}{*}{ 146: 6} & Upper $1 / 2$ & $2.240 \mathrm{E}+05$ & $2.230 \mathrm{E}+05$ & $2.235 \mathrm{E}+05$ \\
\hline S96T003007 & & Lower $1 / 2$ & $2.180 \mathrm{E}+05$ & $2.260 \mathrm{E}+05$ & $2.220 \overline{\mathrm{E}}+05$ \\
\hline S96T003008 & \multirow[t]{2}{*}{$146: 7$} & Upper $1 / 2$ & $2.790 \mathrm{E}+05$ & $2.740 \mathrm{E}+05$ & $2.765 \mathrm{E}+05$ \\
\hline S96T003009 & & Lower $1 / 2$ & $2.620 \mathrm{E}+05$ & $2.760 \mathrm{E}+05$ & $2.690 \mathrm{E}+05$ \\
\hline S96T003148 & \multirow[t]{2}{*}{ 146: 8} & Upper $1 / 2$ & $2.780 \overline{\mathrm{E}}+05$ & $2.790 \mathrm{E}+05$ & $2.785 \mathrm{E}+05$ \\
\hline S96T003149 & & Lower $1 / 2$ & $2.030 \bar{E}+05$ & $2.150 \mathrm{E}+05$ & $2.090 \mathrm{E}+05$ \\
\hline S96T004180 & \multirow[t]{2}{*}{ 146: 9} & Lower $1 / 2$ & $2.090 \mathrm{E}+05$ & $2.040 \mathrm{E}+05$ & $2.065 \mathrm{E}+05$ \\
\hline S96T003150 & & Upper $1 / 2$ & $1.970 \mathrm{E}+05$ & $2.100 \bar{E}+05$ & $2.035 \bar{E}+05$ \\
\hline S96T003450 & Core 141 & $\begin{array}{l}\text { Solid } \\
\text { composite }\end{array}$ & $3.060 \mathrm{E}+05$ & $2.730 \mathrm{E}+05$ & $2.895 \mathrm{E}+05^{\mathrm{QC}: \mathrm{e}}$ \\
\hline S96T003660 & Core 145 & $\begin{array}{l}\text { Solid } \\
\text { composite }\end{array}$ & $3.680 \mathrm{E}+05$ & $2.480 \overline{\mathrm{E}+05}$ & $3.080 \mathrm{E}+05^{\mathrm{QC}: \mathrm{e}}$ \\
\hline S96T004199 & Core 146 & $\begin{array}{l}\text { Solid } \\
\text { composite }\end{array}$ & $2.440 \mathrm{E}+05$ & $2.500 \mathrm{E}+05$ & $2.470 \mathrm{E}+05$ \\
\hline
\end{tabular}


Table B2-34. Tank 241-U-108 Core Sample Analytical Results: Sodium (ICP). (3 sheets)

\begin{tabular}{|c|c|c|c|c|c|}
\hline $\begin{array}{l}\text { Gurple? } \\
\text { Wumber }\end{array}$ & $\begin{array}{l}\text { Squjolo } \\
\text { incarion }\end{array}$ & $\begin{array}{l}\text { Sample } \\
\text { Eortion. }\end{array}$ & Regill & Bupliente & Mernir \\
\hline \multicolumn{2}{|c|}{ Solids yater digest } & & $49 \mathrm{~g}:$ & (. 1989 & (19g/g \\
\hline S96T003453 & Core 141 & $\begin{array}{l}\text { Solid } \\
\text { composite }\end{array}$ & $2.050 \mathrm{E}+05$ & $2.000 \mathrm{E}+05$ & $2.025 \mathrm{E}+05$ \\
\hline S96T003662 & Core 145 & $\begin{array}{l}\text { Solid } \\
\text { composite }\end{array}$ & $2.270 \overline{\mathrm{E}}+05$ & $2.260 \bar{E}+05$ & $2.265 \mathrm{E}+05$ \\
\hline S96T004202 & Core 146 & $\begin{array}{l}\text { Solid } \\
\text { composite }\end{array}$ & $2.190 \mathrm{E}+05$ & $2.180 \overline{\mathrm{E}}+05$ & $2.185 E+05$ \\
\hline \multicolumn{2}{|c|}{ 1.12 Thaids: } & & 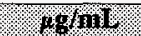 & $\%$ g/nL & $4 \mathrm{~g} / \mathrm{ni}$ I \\
\hline S96T002282 & 141: 1 & $\begin{array}{l}\text { Drainable } \\
\text { liquid }\end{array}$ & $2.220 \mathrm{E}+05$ & $2.240 \mathrm{E}+05$ & $2.230 \mathrm{E}+05^{\mathrm{QC}: \mathrm{d}}$ \\
\hline S96T002942 & 145: 1 & $\begin{array}{l}\text { Drainable } \\
\text { liquid }\end{array}$ & $2.320 \bar{E}+05$ & $2.320 \mathrm{E}+05$ & $2.320 \mathrm{E}+05$ \\
\hline S96T003163 & 146: 1 & $\begin{array}{l}\text { Drainable } \\
\text { liquid }\end{array}$ & $2.330 \mathrm{E}+05$ & $2.230 \mathrm{E}+05$ & $2.280 \mathrm{E}+05$ \\
\hline
\end{tabular}


Table B2-35. Tank 241-U-108 Grab Sample Analytical Results: Sodium (ICP).

\begin{tabular}{|c|c|c|c|c|c|}
\hline Womplo & \%omolo & 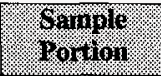 & Resuil & Buplicate & Mern \\
\hline \multicolumn{2}{|c|}{ (1.7. } & & 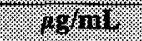 & g gru & 4.8101 \\
\hline S95T000978 & Riser 7 & Grab sample & $3.064 \mathrm{E}+05$ & $2.698 \mathrm{E}+05$ & $2.881 \mathrm{E}+05$ \\
\hline
\end{tabular}

Table B2-36. Tank 241-U-108 Core Sample Analytical Results: Sulfur (ICP).

\begin{tabular}{|c|c|c|c|c|c|}
\hline 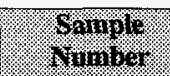 & \% & 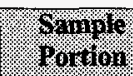 & Prosilin & Bublutise & Mentin \\
\hline \multicolumn{3}{|c|}{ 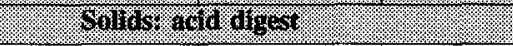 } & 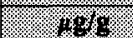 & m9 & (2. \\
\hline S96T003451 & Core 141 & $\begin{array}{l}\text { Solid } \\
\text { composite }\end{array}$ & 4,770 & 4,530 & 4,650 \\
\hline S96T003457 & Core 145 & $\begin{array}{l}\text { Solid } \\
\text { composite }\end{array}$ & 4,670 & 4,790 & 4,730 \\
\hline S96T004200 & Core 146 & $\begin{array}{l}\text { Solid } \\
\text { composite }\end{array}$ & 5,150 & 6,410 & $5,780^{Q \mathrm{C}: \mathrm{a}, \mathrm{c}, \mathrm{e}}$ \\
\hline \multicolumn{2}{|c|}{ 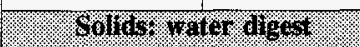 } & & . 496 & 498 & $14 \mathrm{~g}$ \\
\hline S96T003453 & Core 141 & $\begin{array}{l}\text { Solid } \\
\text { composite }\end{array}$ & 5,410 & 5,160 & 5,285 \\
\hline S96T003662 & Core 145 & $\begin{array}{l}\text { Solid } \\
\text { composite }\end{array}$ & 5,620 & 5,170 & 5,395 \\
\hline S96T004202 & Core 146 & $\begin{array}{l}\text { Solid } \\
\text { composite }\end{array}$ & 5,840 & $\overline{5,960}$ & 5,900 \\
\hline \multicolumn{2}{|c|}{ 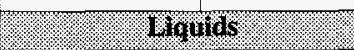 } & & . & $110 \%$ & . $10 \mathrm{~m}$ \\
\hline S96T002282 & 141: 1 & $\begin{array}{l}\text { Drainable } \\
\text { liquid }\end{array}$ & 1,390 & 1,410 & 1,400 \\
\hline S96T002942 & 145: 1 & $\begin{array}{l}\text { Drainable } \\
\text { liquid }\end{array}$ & 1,410 & 1,410 & 1,410 \\
\hline S96T003163 & 146: 1 & $\begin{array}{l}\text { Drainable } \\
\text { liquid }\end{array}$ & 1,450 & 1,390 & 1,420 \\
\hline
\end{tabular}


Table B2-37. Tank 241-U-108 Core Sample Analytical Results: Zinc (ICP).

\begin{tabular}{|c|c|c|c|c|c|}
\hline $\begin{array}{l}\text { Shimple? } \\
\text { Timimer }\end{array}$ & Sample & $\begin{array}{l}\text { Sarrple } \\
\text { Portion }\end{array}$ & Resint & Duplicate & Mein. \\
\hline \multicolumn{2}{|c|}{ S Sollds acid diget } & (1). & 1016 & 19g. & 1356 \\
\hline S96T003451 & Core 141 & $\begin{array}{l}\text { Solid } \\
\text { composite }\end{array}$ & 74.5 & 81.8 & 78.15 \\
\hline S96T003457 & Core 145 & $\begin{array}{l}\text { Solid } \\
\text { composite }\end{array}$ & 40.9 & 41.2 & 41.05 \\
\hline S96T004200 & Core 146 & $\begin{array}{l}\text { Solid } \\
\text { composite }\end{array}$ & 16 & 19.2 & $17.6^{\mathrm{QC}: \mathrm{a}, \mathrm{e}}$ \\
\hline \multicolumn{2}{|c|}{ Solinls rater digect } & & 1488 & 14818 & 148 \\
\hline S96T003453 & Core 141 & $\begin{array}{l}\text { Solid } \\
\text { composite }\end{array}$ & 22.7 & 31.3 & $27^{\mathrm{QC:} \mathrm{e}}$ \\
\hline S96T003662 & Core 145 & $\begin{array}{l}\text { Solid } \\
\text { composite }\end{array}$ & 40.9 & 24.3 & $32.6^{\mathrm{QC:e}}$ \\
\hline S96T004202 & Core 146 & $\begin{array}{l}\text { Solid } \\
\text { composite }\end{array}$ & 10.9 & 10.4 & 10.65 \\
\hline \multicolumn{2}{|c|}{ Whurds: } & 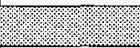 & $16 \% \mathrm{mI}$ & 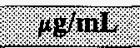 & 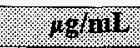 \\
\hline S96T002282 & $141: 1$ & $\begin{array}{l}\text { Drainable } \\
\text { liquid }\end{array}$ & 32.9 & 34.8 & 33.85 \\
\hline S96T002942 & 145: 1 & $\begin{array}{l}\text { Drainable } \\
\text { liquid }\end{array}$ & 56.9 & 60.2 & 58.55 \\
\hline S96T003163 & 146: 1 & $\begin{array}{l}\text { Drainable } \\
\text { liquid }\end{array}$ & 40.7 & 45.7 & $\overline{43.2}$ \\
\hline
\end{tabular}

\section{B2.4.5 Ion Chromatography}

Solid core samples and the core composites were analyzed by IC following water digestion per procedure LA-533-105, Rev. D-1. Liquid core samples and the 1995 grab sample were analyzed directly following dilution per procedure LA-533-105, Rev. C-2.

The analytes of bromide, carbonate, and nitrate were required by the core SAP for solid and core composite samples. Only bromide was required on the drainable liquid samples.

Bromide was measured to evaluate intrusion and contamination of the sample by hydrostatic head fluid or wash water containing LiBr. Carbonate was determined by TIC methodology and not IC (as specified in the core SAP) because the IC eluent is a carbonate buffer. The analytes of chloride, fluoride, phosphate, sulfate, nitrate, and nitrite were required by the grab SAP. The concentrations of anions except bromide by IC are shown in Tables B2-38 
through B2-50. Bromide was not detected in the water-digested solid samples or the drainable liquids. The highest detection limit of the water-digested samples was $1,411 \mu \mathrm{g} / \mathrm{g}$, and the highest detection limit of the drainable liquid samples was $649 \mu \mathrm{g} / \mathrm{mL}$.

For the core samples, additional IC data were collected on an opportunistic basis and are reported here. Because these data were not identified in any DQO document, there were no programmatic QC requirements with respect to these data and the QC information associated with them were not evaluated for this report.

Table B2-38. Tank 241-U-108 Core Sample Analytical Results: Chloride (IC). (3 sheets)

\begin{tabular}{|c|c|c|c|c|c|}
\hline $\begin{array}{l}\text { Simingle } \\
\text { Rumber }\end{array}$ & $\begin{array}{l}\text { Shimple } \\
\text { Encation }\end{array}$ & $\begin{array}{l}\text { Sample } \\
\text { rotifoun }\end{array}$ & resulis & Buplicite & Mrean \\
\hline \multicolumn{2}{|c|}{ Sollos water afgert } & (3) & (36y) & . $198 \%$ : & $10 \%$ \\
\hline \$96T002270 & 141: 1 & Lower $1 / 2$ & 4,214 & 4,150 & 4,182 \\
\hline S96T002273 & 141: 2 & Upper $1 / 2$ & 2,946 & 3,250 & 3,098 \\
\hline S96T002274 & 141: 3 & Upper $1 / 2$ & 3,178 & 3,420 & 3,299 \\
\hline S96T002275 & $141: 3 A$ & Upper $1 / 2$ & 2,936 & 3,100 & 3,018 \\
\hline S96T002276 & \multirow[t]{2}{*}{ 141: 4} & Upper $1 / 2$ & 4,625 & 5,330 & $4,977^{\mathrm{QC}: \mathrm{e}}$ \\
\hline S96T002277 & & Lower $1 / 2$ & 5,478 & 4,760 & $5,119^{\mathrm{QC}: \mathrm{e}}$ \\
\hline S96T002278 & \multirow[t]{2}{*}{$141: 4 \mathrm{~A}$} & Upper $1 / 2$ & 4,790 & 5,290 & $5,0 \overline{40}$ \\
\hline S96T002279 & & Lower $1 / 2$ & 4,731 & 4,580 & 4,655 \\
\hline S96T002617 & \multirow[t]{2}{*}{$141: 5$} & Upper $1 / 2$ & 3,398 & 4,110 & $3,754^{\mathrm{QC}: \mathrm{e}}$ \\
\hline S96T002618 & & Lower $1 / 2$ & 3,093 & 3,050 & 3,071 \\
\hline S96T002619 & \multirow[t]{2}{*}{ 141: 6} & Upper $1 / 2$ & 4,009 & 4,120 & 4,064 \\
\hline S96T002620 & & Lower $1 / 2$ & 3,165 & 3,030 & 3,097 \\
\hline S96T002621 & \multirow[t]{2}{*}{ 141: 7} & Upper $1 / 2$ & 4,806 & 4,740 & 4,773 \\
\hline S96T002622 & & Lower $1 / 2$ & 3,967 & 4,180 & 4,073 \\
\hline S96T002623 & \multirow[t]{2}{*}{$141: 8$} & Upper $1 / 2$ & 4,650 & 4,730 & $4,6 \overline{90}$ \\
\hline S96T002624 & & Lower $1 / 2$ & 1,050 & 1,040 & 1,045 \\
\hline S96T002625 & 141: 9 & Upper $1 / 2$ & 3,922 & 4,040 & 3,981 \\
\hline S96T002924 & $145: 2$ & Upper $1 / 2$ & 2,546 & 2,350 & 2,448 \\
\hline S96T002925 & \multirow[t]{2}{*}{$145: 3$} & Upper $1 / 2$ & 3,461 & 3,370 & 3,415 \\
\hline S96T002926 & & Lower $1 / 2$ & 3,064 & 3,820 & $3,442^{\mathrm{QC}: \mathrm{e}}$ \\
\hline S96T002927 & \multirow[t]{2}{*}{$145: 4$} & Upper $1 / 2$ & 3,717 & 2,710 & $3,21 \overline{3^{\mathrm{QC}: \mathrm{e}}}$ \\
\hline S96T002928 & & Lower $1 / 2$ & 4,011 & 4,050 & 4,030 \\
\hline S96T002929 & \multirow[t]{2}{*}{$145: 5$} & Upper $1 / 2$ & 3,912 & 4,360 & $4,136^{\mathrm{QC}: \mathrm{e}}$ \\
\hline S96T002930 & & Lower $1 / 2$ & 5,278 & 5,560 & $5, \overline{419}$ \\
\hline
\end{tabular}


HNF-SD-WM-ER-639 Rev. 0

Table B2-38. Tank 241-U-108 Core Sample Analytical Results: Chloride (IC). (3 sheets)

\begin{tabular}{|c|c|c|c|c|c|}
\hline $\begin{array}{l}\text { Gimpler. } \\
\text { Cunilorer. }\end{array}$ & C Sample & $\begin{array}{l}\text { Sample } \\
\text { rortion }\end{array}$ & isesill & Hujlicato & Mrean \\
\hline \multicolumn{2}{|c|}{ sollar vafer digert } & : & 1.989 & 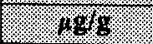 & $.8 \% 8$ \\
\hline S96T002931 & \multirow[t]{2}{*}{$145: 6$} & Upper $1 / 2$ & 3,974 & 4,140 & 4,057 \\
\hline S96T002932 & & Lower $1 / 2$ & 3,317 & 3,690 & $3,503^{Q C: e}$ \\
\hline S96T002933 & \multirow[t]{2}{*}{ 145: 7} & Upper $1 / 2$ & 3,023 & 2,970 & 2,996 \\
\hline S96T002934 & & Lower $1 / 2$ & 2,174 & 2,850 & $2,512^{\mathrm{QC}: \mathrm{e}}$ \\
\hline S96T002935 & \multirow[t]{2}{*}{$145: 8$} & Upper $1 / 2$ & 2,031 & 5,310 & $3,670^{\mathrm{QC}: e^{-}}$ \\
\hline S96T002936 & & Lower $1 / 2$ & $2, \overline{905}$ & 2,650 & 2,777 \\
\hline S96T002937 & \multirow{2}{*}{$145: 9$} & Upper $1 / 2$ & 3,386 & 2,190 & $2,788^{\mathrm{QC}: e}$ \\
\hline S96T002938 & & Lower $1 / 2$ & 3,673 & 1,720 & $2,696^{\mathrm{QC:e}}$ \\
\hline S96T003151 & 146: 1 & Upper $1 / 2$ & 3,714 & 3,750 & 3,732 \\
\hline S96T003152 & \multirow[t]{2}{*}{ 146: 2} & Upper $1 / 2$ & 1,907 & 1,760 & 1,833 \\
\hline S96T003153 & & Lower $1 / 2$ & 2,416 & 1,680 & $2,048^{\mathrm{QC}: \mathrm{e}}$ \\
\hline S96T003154 & \multirow[t]{2}{*}{$146: 3$} & Upper $1 / 2$ & 2,131 & 2,070 & 2,100 \\
\hline S96T003155 & & Lower $1 / 2$ & 1,761 & 1,980 & $1,870^{\overline{Q C: e}}$ \\
\hline S96T003052 & 146: $3 \mathrm{~A}$ & Upper $1 / 2$ & 2,032 & $2, \overline{010}$ & 2,021 \\
\hline S96T003053 & \multirow[t]{2}{*}{$146: 4$} & Upper $1 / 2$ & 2,308 & 2,190 & 2,249 \\
\hline S96T003054 & & Lower $1 / 2$ & 3,894 & 3,940 & 3,917 \\
\hline S96T003055 & \multirow[t]{2}{*}{$146: 5$} & Upper $1 / 2$ & 4,407 & 4,490 & 4,448 \\
\hline S96T003056 & & Lower $1 / 2$ & 3,261 & 3,280 & 3,270 \\
\hline S96T003057 & \multirow[t]{3}{*}{$146: 6$} & Upper $1 / 2$ & 3,620 & 3,460 & 3,540 \\
\hline S96T003058 & & Lower $1 / 2$ & $2,6 \overline{62}$ & 10,000 & $6,331^{\mathrm{QC}: \mathrm{e}}$ \\
\hline S96T004322 & & Lower $1 / 2$ & 3,621 & 3,420 & 3,520 \\
\hline S96T003059 & \multirow[t]{2}{*}{ 146: 7} & Upper $1 / 2$ & 3,470 & 3,490 & 3,480 \\
\hline S96T003060 & & Lower $1 / 2$ & 3,753 & 3,880 & 3,816 \\
\hline S96T003156 & \multirow[t]{2}{*}{$146: 8$} & Upper $1 / 2$ & 3,514 & 3,540 & 3,527 \\
\hline S96T003157 & & Lower $1 / 2$ & 3,523 & 3,590 & 3,556 \\
\hline S96T004181 & \multirow[t]{2}{*}{$146: 9$} & Lower $1 / 2$ & 2,228 & 1,880 & $2,054^{\mathrm{QC}: \mathrm{e}}$ \\
\hline S96T003158 & & Upper $1 / 2$ & 3,044 & 3,130 & 3,087 \\
\hline S96T003452 & Core 141 & $\begin{array}{l}\text { Solid } \\
\text { composite }\end{array}$ & 5,707 & 5,700 & 5,703 \\
\hline S96T003661 & Core 145 & $\begin{array}{l}\text { Solid } \\
\text { composite }\end{array}$ & 3,865 & 3,940 & 3,902 \\
\hline S96T004201 & Core 146 & $\begin{array}{l}\text { Solid } \\
\text { composite }\end{array}$ & 3,540 & 3,510 & 3,525 \\
\hline
\end{tabular}


Table B2-38. Tank 241-U-108 Core Sample Analytical Results: Chloride (IC). (3 sheets)

\begin{tabular}{|c|c|c|c|c|c|}
\hline $\begin{array}{l}\text { Sample } \\
\text { Nuniber }\end{array}$ & $\begin{array}{l}\text { Sampile } \\
\text { Lacition }\end{array}$ & $\begin{array}{l}\text { Sainple } \\
\text { Rortion }\end{array}$ & Resullt & Duplicate & Meari \\
\hline \multicolumn{3}{|c|}{ I. } & 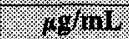 & , & $48 / \mathbf{m i}$ \\
\hline S96T002282 & 141: 1 & $\begin{array}{l}\text { Drainable } \\
\text { liquid }\end{array}$ & 8,598 & 8,800 & 8,699 \\
\hline S96T002942 & $145: 1$ & $\begin{array}{l}\text { Drainable } \\
\text { liquid }\end{array}$ & 9,141 & $9, \overline{140}$ & $9, \overline{140}$ \\
\hline S96T003163 & $146: 1$ & $\begin{array}{l}\text { Drainable } \\
\text { liquid }\end{array}$ & 9,551 & 9,490 & 9,520 \\
\hline
\end{tabular}

Table B2-39. Tank 241-U-108 Grab Sample Analytical Results: Chloride (IC).

\begin{tabular}{|c|c|c|c|c|c|}
\hline Maminger & oramile & 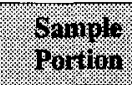 & Ersuly & Bipilicals & Mear \\
\hline \multicolumn{2}{|c|}{ Winasis } & & $40 \% 11$ & 61111 & 48401 \\
\hline S95T000978 & Riser 7 & $\begin{array}{l}\text { Grab } \\
\text { sample }\end{array}$ & 8,270 & 8,560 & 8,415 \\
\hline
\end{tabular}


Table B2-40. Tank 241-U-108 Core Sample Analytical Results: Fluoride (IC). (2 sheets)

\begin{tabular}{|c|c|c|c|c|c|}
\hline $\begin{array}{l}\text { Simingle: } \\
\text { Vimnier. }\end{array}$ & $\begin{array}{l}\text { Gample } \\
\text { Eocation }\end{array}$ & $\begin{array}{l}\text { Sample } \\
\text { Srortion }\end{array}$ & Resinil & Mipliente & Mean \\
\hline \multicolumn{2}{|c|}{ 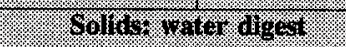 } & (2) & 6.489 & 1.86g & 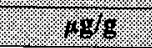 \\
\hline S96T002270 & 141: 1 & Lower $1 / 2$ & 1,525 & 1,510 & 1,517 \\
\hline S96T002273 & $141: 2$ & Upper $1 / 2$ & 548.8 & 600 & $5 \overline{74.4}$ \\
\hline S96T002274 & $141: 3$ & Upper $1 / 2$ & $<122.1$ & $<120$ & $<121.1$ \\
\hline S96T002275 & 141: $3 A$ & Upper $1 / 2$ & $<123.7$ & $<123$ & $<123.4$ \\
\hline S96T002276 & \multirow[t]{2}{*}{ 141: 4} & Upper $1 / 2$ & $<123.1$ & $<126$ & $<124.6$ \\
\hline S96T002277 & & Lower $1 / 2$ & $<117.2$ & $<114$ & $<115.6$ \\
\hline S96T002278 & \multirow[t]{2}{*}{ 141: $4 \mathrm{~A}$} & Upper $1 / 2$ & $<116.8$ & $<118$ & $<117.4$ \\
\hline S96T002279 & & Lower $1 / 2$ & $<113.5$ & $<\overline{113}$ & $<113.3$ \\
\hline S96T002617 & \multirow[t]{2}{*}{$141: 5$} & Upper $1 / 2$ & 558.1 & 576 & 567.1 \\
\hline S96T002618 & & Lower $1 / 2$ & 499.7 & 469 & 484.4 \\
\hline S96T002619 & \multirow[t]{2}{*}{ 141: 6} & Upper $1 / 2$ & $<115.6$ & $<105$ & $<110.3$ \\
\hline S96T002620 & & Lower $1 / 2$ & 535.9 & 549 & 542.4 \\
\hline S96T002621 & \multirow[t]{2}{*}{ 141: 7} & Upper $1 / 2$ & 878.2 & 886 & 882.1 \\
\hline S96T002622 & & Lower $1 / 2$ & 448.4 & $<114$ & $<281.2^{\mathrm{QC}: \mathrm{e}}$ \\
\hline S96T002623 & \multirow[t]{2}{*}{ 141: 8} & Upper $1 / 2$ & $<104.6$ & $<110$ & $<107.3$ \\
\hline S96T002624 & & Lower $1 / 2$ & 632.4 & 4,020 & $2,326^{\mathrm{QC}: \mathrm{e}}$ \\
\hline S96T002625 & $141: 9$ & Upper $1 / 2$ & 2,904 & 2,760 & 2,832 \\
\hline S96T002924 & $145: 2$ & Upper $1 / 2$ & 576.3 & 571 & 573.6 \\
\hline S96T002925 & \multirow[t]{2}{*}{$145: 3$} & Upper $1 / 2$ & 770.9 & 719 & 744.9 \\
\hline S96T002926 & & Lower $1 / 2$ & 587.5 & $<141$ & $<364.2^{\mathrm{QC}: \mathrm{e}}$ \\
\hline S96T002927 & \multirow[t]{2}{*}{$145: 4$} & Upper $1 / 2$ & $<130.8$ & $<129$ & $<129.9$ \\
\hline S96T002928 & & Lower $1 / 2$ & $<105.6$ & $<104$ & $<104.8$ \\
\hline S96T002929 & \multirow[t]{2}{*}{$145: 5$} & Upper $1 / 2$ & $<105.8$ & $<108$ & $<106.9$ \\
\hline S96T002930 & & Lower $1 / 2$ & 882.8 & 954 & 918.4 \\
\hline S96T002931 & \multirow[t]{2}{*}{$145: 6$} & Upper 1/2 & 1,063 & 1,070 & 1,066 \\
\hline S96T002932 & & Lower $1 / 2$ & 868.7 & 839 & 853.8 \\
\hline S96T002933 & \multirow[t]{2}{*}{ 145: 7} & Upper $1 / 2$ & 938.6 & 937 & 937.8 \\
\hline S96T002934 & & Lower $1 / 2$ & 740.6 & 839 & $789.8^{\mathrm{QC}: \mathrm{e}}$ \\
\hline S96T002935 & \multirow[t]{2}{*}{$145: 8$} & Upper $1 / 2$ & 859.8 & 835 & 847.4 \\
\hline S96T002936 & & Lower $1 / 2$ & $<104$ & $<104$ & $<104$ \\
\hline S96T002937 & \multirow[t]{2}{*}{$145: 9$} & Upper $1 / 2$ & 3,159 & 2,310 & $2,734^{\mathrm{QC}: e}$ \\
\hline S96T002938 & & Lower $1 / 2$ & 2,728 & 2,620 & 2,674 \\
\hline
\end{tabular}


Table B2-40. Tank 241-U-108 Core Sample Analytical Results: Fluoride (IC). (2 sheets)

\begin{tabular}{|c|c|c|c|c|c|}
\hline Shmole & S Samplention & $\begin{array}{l}\text { Sanple } \\
\text { Purtion }\end{array}$ & Iresint & Shipiliento & Mearia \\
\hline \multicolumn{2}{|c|}{ 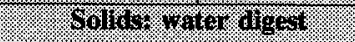 } & 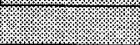 & .498 & 1.969 & $1.78 \mathrm{~g} / \mathrm{s}$ \\
\hline S96T003151 & 146: 1 & Upper $1 / 2$ & $<104$ & $<101$ & $<102.5$ \\
\hline S96T003152 & \multirow[t]{2}{*}{ 146: 2} & Upper $1 / 2$ & $<101.3$ & $<106$ & $<103.6$ \\
\hline S96T003153 & & Lower $1 / 2$ & 819.3 & 818 & 818.6 \\
\hline S96T003154 & \multirow[t]{2}{*}{$146: 3$} & Upper $1 / 2$ & 583.7 & 554 & 568.8 \\
\hline S96T003155 & & Lower $1 / 2$ & $<167.4$ & 802 & $<484.7^{\mathrm{QC}: \mathrm{e}}$ \\
\hline S96T003052 & 146: $3 \mathrm{~A}$ & Upper $1 / 2$ & 865.5 & 957 & $911.2^{\mathrm{QC}: \mathrm{e}}$ \\
\hline S96T003053 & \multirow[t]{2}{*}{ 146: 4} & Upper $1 / 2$ & 1,131 & 1,070 & 1,100 \\
\hline S96T003054 & & Lower $1 / 2$ & 1,534 & 1,590 & 1,562 \\
\hline S96T003055 & \multirow[t]{2}{*}{$146: 5$} & Upper $1 / 2$ & 1,685 & 1,770 & 1,727 \\
\hline S96T003056 & & Lower $1 / 2$ & 1,344 & 1,260 & 1,302 \\
\hline S96T003057 & \multirow[t]{3}{*}{ 146: 6} & Upper $1 / 2$ & 1,557 & 1,350 & $1,453^{\mathrm{QC}: \mathrm{e}}$ \\
\hline S96T003058 & & Lower $1 / 2$ & 1,205 & 1,580 & $1,392^{\mathrm{QC}: \mathrm{e}}$ \\
\hline S96T004322 & & Lower $1 / 2$ & 645.2 & 609 & 627.1 \\
\hline S96T003059 & \multirow[t]{2}{*}{$146: 7$} & Upper $1 / 2$ & 1,615 & 1,640 & 1,627 \\
\hline S96T003060 & & Lower $1 / 2$ & 2,213 & 2,300 & 2,256 \\
\hline S96T003156 & \multirow[t]{2}{*}{$146: 8$} & Upper $1 / 2$ & 1,715 & 1,680 & 1,697 \\
\hline S96T003157 & & Lower $1 / 2$ & 2,027 & 2,060 & 2,043 \\
\hline S96T004181 & \multirow[t]{2}{*}{ 146: 9} & Lower $1 / 2$ & 2,074 & 2,410 & $2,242^{\mathrm{QC:c}}$ \\
\hline S96T003158 & & Upper $1 / 2$ & 3,512 & 3,540 & 3,526 \\
\hline S96T003452 & Core 141 & \begin{tabular}{|l|}
$\begin{array}{l}\text { Solid } \\
\text { composite }\end{array}$ \\
\end{tabular} & $<54.59$ & $<54.5$ & $<54.55$ \\
\hline S96T003661 & Core 145 & $\begin{array}{l}\text { Solid } \\
\text { composite }\end{array}$ & $<97.76$ & $<75.6$ & $<86.68^{\mathrm{QC}: \mathrm{e}}$ \\
\hline S96T004201 & Core 146 & $\begin{array}{l}\text { Solid } \\
\text { composite }\end{array}$ & 570.4 & 577 & 573.7 \\
\hline \multicolumn{2}{|c|}{ Gropids } & 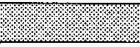 & 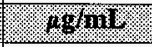 & 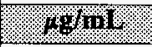 & (19garl \\
\hline S96T002282 & $141: 1$ & $\begin{array}{l}\text { Drainable } \\
\text { liquid }\end{array}$ & $<53.83$ & $<53.8$ & $<53.82$ \\
\hline S96T002942 & 145: 1 & $\begin{array}{l}\text { Drainable } \\
\text { liquid }\end{array}$ & $<53.83$ & $<53.8$ & $<53.82$ \\
\hline S96T003163 & 146: 1 & $\begin{array}{l}\text { Drainable } \\
\text { liquid }\end{array}$ & 804.2 & 776 & 790.1 \\
\hline
\end{tabular}


Table B2-41. Tank 241-U-108 Grab Sample Analytical Results: Fluoride (IC).

\begin{tabular}{|c|c|c|c|c|c|}
\hline 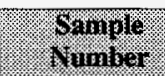 & 6ograpon & 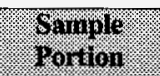 & Resill & Buplinatio & Wean \\
\hline & 16s & & \% 411 & , $97 \mathrm{ml}$ & $796 / 110$ \\
\hline S95T000978 & Riser 7 & Grab sample & 1,130 & 1,110 & 1,120 \\
\hline
\end{tabular}

Table B2-42. Tank 241-U-108 Core Sample Analytical Results: Nitrate (IC). (3 sheets)

\begin{tabular}{|c|c|c|c|c|c|}
\hline \% & $\frac{9}{404010}$ & (4) & 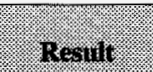 & $314018 \% 2 \%$ & 16an. \\
\hline \multicolumn{2}{|c|}{ (1) } & & (2) & 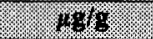 & $1 \% 48$ \\
\hline S96T002270 & 141: 1 & Lower $1 / 2$ & 94,880 & 96,100 & 95,490 \\
\hline S96T002273 & $141: 2$ & Upper $1 / 2$ & $3.451 \mathrm{E}+05$ & $3.440 \mathrm{E}+05$ & $3.446 \mathrm{E}+05$ \\
\hline S96T002274 & $141: 3$ & Upper $1 / 2$ & $4.273 \mathrm{E}+05$ & $4.490 \mathrm{E}+05$ & $4.382 \mathrm{E}+05$ \\
\hline S96T002275 & $141: 3 \AA$ & Upper $1 / 2$ & $4.571 \bar{E}+05$ & $4.320 \mathrm{E}+05$ & $4.446 \mathrm{E}+05$ \\
\hline S96T002276 & \multirow[t]{2}{*}{$141: 4$} & Upper $1 / 2$ & $3.4 \overline{98 \mathrm{E}}+05$ & $2.950 \mathrm{E}+05$ & $3.224 \mathrm{E}+05^{\mathrm{QC}: \mathrm{e}}$ \\
\hline S96T002277 & & Lower $1 / 2$ & $2.813 \mathrm{E}+05$ & $3.120 E+05$ & $2.967 \mathrm{E}+05^{\mathrm{QC} e \mathrm{e}}$ \\
\hline S96T002278 & \multirow[t]{2}{*}{$141: 4 \mathrm{~A}$} & Upper $1 / 2$ & $3.386 \mathrm{E}+05$ & $2.990 \mathrm{E}+05$ & $3.188 \mathrm{E}+05^{\mathrm{QC}: \mathrm{E}}$ \\
\hline S96T002279 & & Lower $1 / 2$ & $3.482 \mathrm{E}+05$ & $3.460 \mathrm{E}+05$ & $3.471 \mathrm{E}+05$ \\
\hline S96T002617 & \multirow[t]{2}{*}{$141: 5$} & Upper $1 / 2$ & $2.475 \mathrm{E}+05$ & $2.370 \mathrm{E}+05$ & $2.423 \mathrm{E}+05$ \\
\hline \$96Г002618 & & Lower $1 / 2$ & $2.901 \mathrm{E}+05$ & $2.990 \mathrm{E}+05$ & $2.946 \mathrm{E}+05$ \\
\hline S96T002619 & \multirow[t]{2}{*}{$141: 6$} & Upper $1 / 2$ & $4.070 \mathrm{E}+05$ & $3.930 \mathrm{E}+05$ & $4.000 \mathrm{E}+05$ \\
\hline S96T002620 & & Lower $1 / 2$ & $3.987 \mathrm{E}+05$ & $4.100 \mathrm{E}+05$ & $4.044 \mathrm{E}+05$ \\
\hline S96T002621 & \multirow[t]{2}{*}{$141: 7$} & Upper $1 / 2$ & $1.316 \mathrm{E}+05$ & $1.320 \mathrm{E}+05$ & $1.318 \mathrm{E}+05$ \\
\hline \$96T002622 & & Lower $1 / 2$ & $2.407 \mathrm{E}+05$ & $2.260 \mathrm{E}+05$ & $2.334 \mathrm{E}+05$ \\
\hline S96T002623 & \multirow[t]{2}{*}{$141: 8$} & Upper $1 / 2$ & $1.137 \mathrm{E}+05$ & $1.160 \mathrm{E}+05$ & $1.149 \mathrm{E}+05$ \\
\hline S96T002624 & & Lower $1 / 2$ & 26,920 & 29,900 & $28,410^{\mathrm{QC}: \mathrm{e}}$ \\
\hline S96T002625 & $141: 9$ & Upper $1 / 2$ & $1.722 \mathrm{E}+05$ & $1.580 \mathrm{E}+05$ & $1.651 \mathrm{E}+05$ \\
\hline S96T002924 & $145: 2$ & Upper $1 / 2$ & $4.511 \mathrm{E}+05$ & $4.770 \mathrm{E}+05$ & $4.641 \mathrm{E}+05$ \\
\hline S96T002925 & \multirow[t]{2}{*}{$145: 3$} & Upper $1 / 2$ & $4.045 E+05$ & $4.100 \mathrm{E}+05$ & $4.073 \mathrm{E}+05$ \\
\hline S96T002926 & & Lower $1 / 2$ & $4.079 \mathrm{E}+05$ & $4.010 \mathrm{E}+05$ & $4.045 E+05$ \\
\hline S96T002927 & \multirow[t]{2}{*}{$145: 4$} & Upper $1 / 2$ & $4.339 \mathrm{E}+05$ & $4.520 \mathrm{E}+05$ & $4.430 \mathrm{E}+05$ \\
\hline S96T002928 & & Lower $1 / 2$ & $3.389 \mathrm{E}+05$ & $3.380 \mathrm{E}+05$ & $3.385 \mathrm{E}+05$ \\
\hline S96T002929 & \multirow[t]{2}{*}{$145: 5$} & Upper $1 / 2$ & $3.593 \mathrm{E}+05$ & $3.540 \mathrm{E}+05$ & $3.567 \mathrm{E}+05$ \\
\hline S96T002930 & & Lower $1 / 2$ & $2.012 \mathrm{E}+05$ & $1.910 \mathrm{E}+05$ & $1.961 \mathrm{E}+05$ \\
\hline
\end{tabular}


Table B2-42. Tank 241-U-108 Core Sample Analytical Results: Nitrate (IC). (3 sheets)

\begin{tabular}{|c|c|c|c|c|c|}
\hline 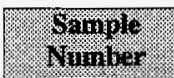 & 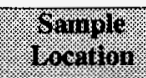 & 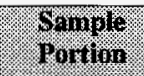 & Tresilit & Oriplicate & Nentr \\
\hline \multicolumn{2}{|c|}{ V. } & & 1.96 & 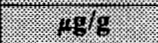 & (1) \\
\hline S96T002931 & \multirow[t]{2}{*}{$145: 6$} & Upper $1 / 2$ & $2.753 \mathrm{E}+05$ & $2.550 \mathrm{E}+05$ & $2.652 \mathrm{E}+05$ \\
\hline S96T002932 & & Lower $1 / 2$ & $3.094 \mathrm{E}+05$ & $3.150 \mathrm{E}+05$ & $3.122 \mathrm{E}+05$ \\
\hline S96T002933 & \multirow[t]{2}{*}{$145: 7$} & Upper 1/2 & $2.669 \mathrm{E}+05$ & $2.700 \mathrm{E}+05$ & $2.685 \mathrm{E}+05$ \\
\hline S96T002934 & & Lower $1 / 2$ & $1.452 \mathrm{E}+05$ & $1.700 \mathrm{E}+05$ & $1.5 \overline{76 \mathrm{E}+05^{\mathrm{QC:e}}}$ \\
\hline S96T002935 & \multirow[t]{2}{*}{ 145: 8} & Upper $1 / 2$ & $4.106 \mathrm{E}+05$ & $3.580 \mathrm{E}+05$ & $3.843 \mathrm{E}+05^{\mathrm{QC}: \mathrm{e}}$ \\
\hline S96T002936 & & Lower $1 / 2$ & $2.810 \mathrm{E}+05$ & $3.050 \mathrm{E}+05$ & $2.930 \mathrm{E}+05$ \\
\hline S96T002937 & \multirow[t]{2}{*}{ 145: 9} & Upper $1 / 2$ & $1.153 \mathrm{E}+05$ & 73,100 & $94 \overline{200^{\text {QC:e }}}$ \\
\hline S96T002938 & & Lower $1 / 2$ & 59,620 & 56,400 & $58, \overline{010}$ \\
\hline S96T003151 & 146: 1 & Upper $1 / 2$ & $2.418 \mathrm{E}+05$ & $2.870 \mathrm{E}+05$ & $2.644 \mathrm{E}+05^{\mathrm{QC:B}}$ \\
\hline S96T003152 & \multirow[t]{2}{*}{$146: 2$} & Upper $1 / 2$ & $4 . 5 \longdiv { 4 5 E + 0 5 }$ & $4.750 \mathrm{E}+05$ & $4.648 \mathrm{E}+05$ \\
\hline S96T003153 & & Lower $1 / 2$ & $5.549 \mathrm{E}+05$ & $5.420 \mathrm{E}+05$ & $5.485 \mathrm{E}+05$ \\
\hline S96T003154 & \multirow[t]{2}{*}{$146: 3$} & Upper $1 / 2$ & $4.192 \mathrm{E}+05$ & $4.430 \mathrm{E}+05$ & $4.311 \mathrm{E}+05$ \\
\hline S96T003155 & & Lower $1 / 2$ & $5.212 \mathrm{E}+05$ & $5.220 \mathrm{E}+05$ & $5.216 \mathrm{E}+05$ \\
\hline S96T003052 & 146: $3 \mathrm{~A}$ & Upper $1 / 2$ & $4.680 \mathrm{E}+05$ & $4.860 \mathrm{E}+05$ & $4.770 \mathrm{E}+05$ \\
\hline S96T003053 & \multirow[t]{2}{*}{$146: 4$} & Upper $1 / 2$ & $4.530 \mathrm{E}+05$ & $4.590 \mathrm{E}+05$ & $4.560 \mathrm{E}+05$ \\
\hline S96T003054 & & Lower $1 / 2$ & $2.985 \mathrm{E}+05$ & $3.030 \mathrm{E}+05$ & $3.008 \mathrm{E}+05$ \\
\hline S96T003055 & \multirow[t]{2}{*}{$146: 5$} & Upper $1 / 2$ & $2.400 \mathrm{E}+05$ & $2.450 \mathrm{E}+05$ & $2 . \overline{425 E+05}$ \\
\hline S96T003056 & & Lower $1 / 2$ & $3.549 \mathrm{E}+05$ & $3.610 \mathrm{E}+05$ & $3.580 \mathrm{E}+05$ \\
\hline S96T003057 & \multirow[t]{3}{*}{$146: 6$} & Upper $1 / 2$ & $3.189 \mathrm{E}+05$ & $3.110 \mathrm{E}+05$ & $3.150 \overline{\mathrm{E}}+05$ \\
\hline S96T003058 & & Lower $1 / 2$ & $2.108 \mathrm{E}+05$ & $2.760 \mathrm{E}+05$ & $2.434 \mathrm{E}+05^{\mathrm{QCic}}$ \\
\hline S96T004322 & & Lower $1 / 2$ & $3.350 \mathrm{E}+05$ & $3.150 \mathrm{E}+05$ & $3.250 \mathrm{E}+05$ \\
\hline S96T003059 & \multirow[t]{2}{*}{$146: 7$} & Upper $1 / 2$ & $2.243 \mathrm{E}+05$ & $2.230 \mathrm{E}+05$ & $2.237 \bar{E}+05$ \\
\hline S96T003060 & & Lower $1 / 2$ & $1.110 \mathrm{E}+05$ & $1.090 \mathrm{E}+05$ & $1.100 \mathrm{E}+05$ \\
\hline S96T003156 & \multirow[t]{2}{*}{ 146: 8} & Upper $1 / 2$ & $1.654 \mathrm{E}+05$ & $1.630 \mathrm{E}+05$ & $1.642 \mathrm{E}+05$ \\
\hline S96T003157 & & Lower $1 / 2$ & $1.089 \mathrm{E}+05$ & $1.020 \mathrm{E}+05$ & $1.055 \mathrm{E}+05$ \\
\hline S96T004181 & \multirow[t]{2}{*}{ 146: 9} & Lower $1 / 2$ & 61,370 & 52,500 & $56, \overline{935^{\mathrm{QC}: \mathrm{e}}}$ \\
\hline S96T003158 & & Upper $1 / 2$ & $1.022 \mathrm{E}+05$ & 99,900 & $1.011 \mathrm{E}+05$ \\
\hline S96T003452 & Core $1 \overline{41}$ & $\begin{array}{l}\text { Solid } \\
\text { composite }\end{array}$ & $1.504 \mathrm{E}+05$ & $1.420 \mathrm{E}+05$ & $1.462 \mathrm{E}+05$ \\
\hline S96T003661 & Core 145 & $\begin{array}{l}\text { Solid } \\
\text { composite }\end{array}$ & $3.232 \mathrm{E}+05$ & $3.240 \mathrm{E}+05$ & $3.236 \mathrm{E}+05$ \\
\hline S96T004201 & Core 146 & $\begin{array}{l}\text { Solid } \\
\text { composite }\end{array}$ & $3.002 \mathrm{E}+05$ & $2.980 \mathrm{E}+05$ & $2.991 \mathrm{E}+05$ \\
\hline
\end{tabular}


Table B2-42. Tank 241-U-108 Core Sample Analytical Results: Nitrate (IC). (3 sheets)

\begin{tabular}{|c|c|c|c|c|c|}
\hline $\begin{array}{l}\text { Gumples. } \\
\text { Number }\end{array}$ & $\begin{array}{l}\text { Somple. } \\
\text { Eocation }\end{array}$ & $\begin{array}{l}\text { Somple } \\
\text { Partion }\end{array}$ & Ressilt & Duplicate & Mean \\
\hline \multicolumn{2}{|c|}{ (1) Siquirs: } & ? & aginis & thror & \%g/mit. \\
\hline S96T002282 & 141: 1 & $\begin{array}{l}\text { Drainable } \\
\text { liquid }\end{array}$ & $1.738 \mathrm{E}+05$ & $1.740 \mathrm{E}+05$ & $1.739 \mathrm{E}+05^{\mathrm{QC}: \mathrm{c}}$ \\
\hline S96T002942 & $145: 1$ & $\begin{array}{l}\text { Drainable } \\
\text { liquid }\end{array}$ & $1.825 \mathrm{E}+05$ & $1.830 \mathrm{E}+05$ & $1.828 \mathrm{E}+05^{\mathrm{QC}: c}$ \\
\hline S96T003163 & $146: 1$ & $\begin{array}{l}\text { Drainable } \\
\text { liquid }\end{array}$ & $1.951 \mathrm{E}+05$ & $1.950 \mathrm{E}+05$ & $1.951 \mathrm{E}+05$ \\
\hline
\end{tabular}

Table B2-43. Tank 241-U-108 Grab Sample Analytical Results: Nitrate (IC).

\begin{tabular}{|c|c|c|c|c|c|}
\hline Srenple & $\begin{array}{l}\text { Grouje } \\
\text { gocatlon }\end{array}$ & $\begin{array}{l}\text { Shingle } \\
\text { Iortion }\end{array}$ & Resin: & Buplicate & Mean \\
\hline & ivi : & & 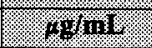 & . & U. $1 \mathrm{~g} / \mathrm{mi}$ \\
\hline S95T000978 & Riser 7 & Grab sample & $1.730 \mathrm{E}+05$ & $1.740 \mathrm{E}+05$ & $1.735 E+05$ \\
\hline
\end{tabular}


HNF-SD-WM-ER-639 Rev. 0

Table B2-44. Tank 241-U-108 Core Sample Analytical Results: Nitrite (IC). (2 sheets)

\begin{tabular}{|c|c|c|c|c|c|}
\hline $\begin{array}{l}\text { Gomple. } \\
\text { number }\end{array}$ & 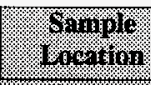 & $\begin{array}{l}\text { STiniele } \\
\text { Portion }\end{array}$ & Resuly & Buplicitis. & Mrean \\
\hline \multicolumn{2}{|c|}{ 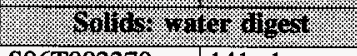 } & & 1089 & (ygge & (1.89 \\
\hline \$96T002270 & 141: 1 & Lower $1 / 2$ & 63,020 & 62,700 & 62,860 \\
\hline S96T002273 & 141: 2 & Upper $1 / 2$ & 44,920 & 49,000 & 46,960 \\
\hline S96T002274 & $141: 3$ & Upper $1 / 2$ & 42,760 & 45,200 & 43,980 \\
\hline S96T002275 & 141: $3 \mathrm{~A}$ & Upper $1 / 2$ & 38,340 & 41,000 & 39,670 \\
\hline S96T002276 & \multirow[t]{2}{*}{ 141: 4} & Upper $1 / 2$ & 61,530 & 69,100 & $65,310^{\mathrm{oC}: \mathrm{e}}$ \\
\hline S96T002277 & & Lower $1 / 2$ & 72,650 & 63,300 & $67,970^{\mathrm{oC}: \mathrm{e}}$ \\
\hline S96T002278 & \multirow[t]{2}{*}{$141: 4 \mathrm{~A}$} & Upper $1 / 2$ & 59,750 & 69,400 & $64,570^{\mathrm{oC}: \mathrm{e}}$ \\
\hline S96T002279 & & Lower $1 / 2$ & 62,070 & 61,200 & 61,630 \\
\hline S96T002617 & \multirow[t]{2}{*}{$141: 5$} & Upper $1 / 2$ & 48,820 & 49,200 & 49,010 \\
\hline S96T002618 & & Lower $1 / 2$ & 41,550 & 39,600 & 40,570 \\
\hline S96T002619 & \multirow[t]{2}{*}{$141: 6$} & Upper $1 / 2$ & 45,570 & 46,800 & 46,180 \\
\hline S96T002620 & & Lower $1 / 2$ & 41,760 & 40,000 & 40,880 \\
\hline S96T002621 & \multirow[t]{2}{*}{ 141: 7} & Upper $1 / 2$ & 72,490 & 71,700 & 72,090 \\
\hline S96T002622 & & Lower $1 / 2$ & 59,750 & 62,700 & 61,220 \\
\hline S96T002623 & \multirow[t]{2}{*}{ 141: 8} & Upper $1 / 2$ & 74,360 & 76,100 & 75,230 \\
\hline S96T002624 & & Lower $1 / 2$ & 14,440 & 14,900 & 14,670 \\
\hline S96T002625 & $141: 9$ & Upper $1 / 2$ & 60,030 & 63,800 & 61,910 \\
\hline S96T002924 & $145: 2$ & Upper $1 / 2$ & 37,520 & 34,000 & 35,760 \\
\hline S96T002925 & \multirow[t]{2}{*}{$145: 3$} & Upper $1 / 2$ & 48,070 & 45,600 & 46,830 \\
\hline S96T002926 & & Lower $1 / 2$ & 42,530 & 49,800 & $46,160^{\mathrm{QC}: \mathrm{e}}$ \\
\hline S96T002927 & \multirow[t]{2}{*}{$145: 4$} & Upper $1 / 2$ & 44,820 & 40,400 & $42,610^{\mathrm{QC}: \mathrm{e}}$ \\
\hline S96T002928 & & Lower $1 / 2$ & 56,530 & 56,300 & 56,410 \\
\hline S96T002929 & \multirow[t]{2}{*}{$145: 5$} & Upper $1 / 2$ & 49,700 & 52,000 & 50,850 \\
\hline S96T002930 & & Lower $1 / 2$ & 66,950 & 70,000 & 68,470 \\
\hline S96T002931 & \multirow[t]{2}{*}{$145: 6$} & Upper $1 / 2$ & 53,460 & 57,100 & 55,280 \\
\hline S96T002932 & & Lower $1 / 2$ & 49,930 & 49,100 & 49,510 \\
\hline S96T002933 & \multirow[t]{2}{*}{$145: 7$} & Upper $1 / 2$ & 53,620 & 53,200 & $\overline{53,410}$ \\
\hline S96T002934 & & Lower $1 / 2$ & 39,220 & 51,600 & $45,410^{\text {कC:e }}$ \\
\hline S96T002935 & \multirow[t]{2}{*}{$145: 8$} & Upper $1 / 2$ & 37,790 & 35,200 & 36,490 \\
\hline S96T002936 & & Lower $1 / 2$ & 48,640 & 44,200 & 46,420 \\
\hline S96T002937 & \multirow[t]{2}{*}{$145: 9$} & Upper $1 / 2$ & 56,020 & 35,900 & $45,960^{\text {QC:e }}$ \\
\hline S96T002938 & & Lower $1 / 2$ & 32,840 & 31,600 & 32,220 \\
\hline
\end{tabular}


Table B2-44. Tank 241-U-108 Core Sample Analytical Results: Nitrite (IC). (2 sheets)

\begin{tabular}{|c|c|c|c|c|c|}
\hline (6) & \% & 3011004 & Resull: & Ouplingro & Hean \\
\hline \multicolumn{2}{|c|}{ 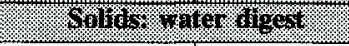 } & & 40 & 169 & $1 \% f_{9}$ \\
\hline S96T003151 & $146: 1$ & Upper $1 / 2$ & 53,140 & 53,300 & 53,220 \\
\hline S96T003152 & \multirow{2}{*}{ 146: 2} & Upper $1 / 2$ & 32,760 & 29,900 & 31,330 \\
\hline S96T003153 & & Lower $1 / 2$ & 25,800 & 27,100 & 26,450 \\
\hline S96T003154 & \multirow{2}{*}{ 146: 3} & Upper $1 / 2$ & 32,590 & 33,300 & 32,940 \\
\hline S96T003155 & & Lower $1 / 2$ & 31,320 & 33,200 & 32,260 \\
\hline S96T003052 & 146: $3 A$ & Upper $1 / 2$ & 34,790 & 32,500 & 33,640 \\
\hline S96T003053 & \multirow[t]{2}{*}{ 146: 4} & Upper $1 / 2$ & 39,250 & 37,900 & 38,570 \\
\hline S96T003054 & & Lower $1 / 2$ & 54,410 & 56,000 & 55,200 \\
\hline \$96T003055 & \multirow[t]{2}{*}{$146: 5$} & Upper $1 / 2$ & 57,440 & 58,600 & 58,020 \\
\hline S96T003056 & & Lower $1 / 2$ & 45,070 & 46,800 & 45,930 \\
\hline S96T003057 & \multirow[t]{3}{*}{ 146: 6} & Upper $1 / 2$ & 51,400 & 50,700 & 51,050 \\
\hline S96T003058 & & Lower $1 / 2$ & 44,910 & 55,200 & $50,050^{\mathrm{QC}: e}$ \\
\hline S96T004322 & & Lower $1 / 2$ & 55,270 & 55,900 & 55,580 \\
\hline S96T003059 & \multirow[t]{2}{*}{ 146: 7} & Upper $1 / 2$ & 64,590 & 64,300 & 64,440 \\
\hline S96T003060 & & Lower $1 / 2$ & 72,400 & 72,600 & 72,500 \\
\hline S96T003156 & \multirow[t]{2}{*}{ 146: 8} & Upper $1 / 2$ & 67,810 & 69,300 & 68,550 \\
\hline S96T003157 & & Lower $1 / 2$ & 69,580 & 71,700 & 70,640 \\
\hline S96T004181 & \multirow[t]{2}{*}{ 146: 9} & Lower $1 / 2$ & 36,330 & 30,800 & $33,560^{\mathrm{QC}: e}$ \\
\hline S96T003158 & & Upper $1 / 2$ & 58,460 & 59,600 & 59,030 \\
\hline S96T003452 & Core 141 & $\begin{array}{l}\text { Solid } \\
\text { composite }\end{array}$ & 82,370 & 83,000 & 82,680 \\
\hline S96T003661 & Core 145 & $\begin{array}{l}\text { Solid } \\
\text { composite }\end{array}$ & 55,920 & 53,000 & 54,460 \\
\hline S96T004201 & Core 146 & $\begin{array}{l}\text { Solid } \\
\text { composite }\end{array}$ & 54,110 & 53,900 & 54,000 \\
\hline 1. & $18 \mathrm{~s}$. & 2. & $4.11 \%$ & 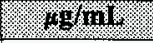 & 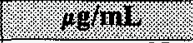 \\
\hline S96T002282 & 141: 1 & $\begin{array}{l}\text { Drainable } \\
\text { liquid }\end{array}$ & $1.311 \mathrm{E}+05$ & $1.310 \mathrm{E}+05$ & $1.311 \mathrm{E}+05^{\mathrm{QC:c}}$ \\
\hline S96T002942 & 145: 1 & $\begin{array}{l}\text { Drainable } \\
\text { liquid }\end{array}$ & $1.345 E+05$ & $1.360 \mathrm{E}+05$ & $1.353 \mathrm{E}+05^{\mathrm{QC}: \mathrm{c}}$ \\
\hline S96T003163 & 146: 1 & $\begin{array}{l}\text { Drainable } \\
\text { liquid }\end{array}$ & $1.359 \mathrm{E}+05$ & $1.370 \mathrm{E}+05$ & $1.365 \mathrm{E}+05$ \\
\hline
\end{tabular}


Table B2-45. Tank 241-U-108 Grab Sample Analytical Results: Nitrite (IC).

\begin{tabular}{|c|c|c|c|c|c|}
\hline owing & 6\%oring & 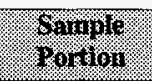 & Result & Buplicarto & 18an \\
\hline \multicolumn{2}{|c|}{ Wuids } & & 4o 4 ing & o. & W9611 \\
\hline S95T000978 & Riser 7 & Grab sample & $1.160 \mathrm{E}+05$ & $1.150 \mathrm{E}+05$ & $1.155 \mathrm{E}+05$ \\
\hline
\end{tabular}

Table B2-46. Tank 241-U-108 Core Sample Analytical Results: Phosphate (IC).

(3 sheets)

\begin{tabular}{|c|c|c|c|c|c|}
\hline $\begin{array}{l}\text { Sumple } \\
\text { Nimbor. }\end{array}$ & $\begin{array}{l}\text { Simple } \\
\text { Irection }\end{array}$ & $\begin{array}{l}\text { Sample } \\
\text { Portion. }\end{array}$ & Resul: & Buplicate & Hew1. \\
\hline \multicolumn{2}{|c|}{ 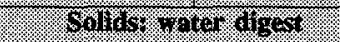 } & 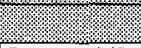 & 1.26 & $12 \mathrm{gla}^{2}$ & . $4 \mathrm{~g} / \mathrm{g}$ \\
\hline S96T002270 & 141: 1 & Lower $1 / 2$ & 40,710 & 40,600 & 40,650 \\
\hline S96T002273 & $141: 2$ & Upper $1 / 2$ & 7,911 & 8,250 & 8,080 \\
\hline S96T002274 & $141: 3$ & Upper $1 / 2$ & $3,67 \overline{7}$ & 4,030 & $3,8 \overline{53}$ \\
\hline S96T002275 & $141: 3 \mathrm{~A}$ & Upper $1 / 2$ & 3,640 & 2,640 & $3,140^{\mathrm{QC}: \mathrm{e}}$ \\
\hline S96T002276 & \multirow[t]{2}{*}{ 141: 4} & Upper $1 / 2$ & 6,919 & 6,590 & $6, \overline{754}$ \\
\hline S96T002277 & & Lower $1 / 2$ & 4,613 & $4, \overline{440}$ & 4,526 \\
\hline S96T002278 & \multirow[t]{2}{*}{$141: 4 \mathrm{~A}$} & Upper $1 / 2$ & 4,997 & 4,000 & $4,498^{\mathrm{QC}: \mathrm{c}}$ \\
\hline S96T002279 & & Lower $1 / 2$ & 6,007 & 6,180 & 6,093 \\
\hline S96T002617 & \multirow[t]{2}{*}{ 141: 5} & Upper $1 / 2$ & 5,884 & 6,180 & $6,0 \overline{032}$ \\
\hline S96T002618 & & Lower $1 / 2$ & 4,875 & 5,580 & $5,227^{\mathrm{QC}: \mathrm{e}}$ \\
\hline S96T002619 & \multirow[t]{2}{*}{ 141: 6} & Upper $1 / 2$ & 6,072 & 7,740 & $6,906^{\overline{\mathrm{QC}: \mathrm{e}}}$ \\
\hline S96T002620 & & Lower $1 / 2$ & $6, \overline{155}$ & 5,840 & 5,997 \\
\hline S96T002621 & \multirow[t]{2}{*}{$141: 7$} & Upper $1 / 2$ & 9,486 & 10,000 & 9,743 \\
\hline S96T002622 & & Lower $1 / 2$ & 10,290 & 8,850 & $9,570^{\mathrm{QC}: \bar{e}}$ \\
\hline S96T002623 & \multirow[t]{2}{*}{$141: 8$} & Upper $1 / 2$ & $10,4 \overline{20}$ & 9,680 & 10,050 \\
\hline S96T002624 & & Lower $1 / 2$ & $1.139 \mathrm{E}+05$ & $1.190 \mathrm{E}+05$ & $1.165 E+05$ \\
\hline S96T002625 & $141: 9$ & Upper $1 / 2$ & 40,400 & 37,100 & 38,750 \\
\hline S96T002924 & $1 \overline{45: 2}$ & Upper $1 / 2$ & 3,537 & 5,880 & $4,708^{\mathrm{QC}: \mathrm{e}}$ \\
\hline S96T002925 & \multirow[t]{2}{*}{ 145: $\overline{3}$} & Upper $1 / 2$ & 5,522 & 4,880 & $5,201^{\mathrm{QC}: \mathrm{e}}$ \\
\hline S96T002926 & & Lower $1 / 2$ & 3,648 & 4,130 & $3,889^{\mathrm{QC}: \mathrm{e}}$ \\
\hline S96T002927 & \multirow[t]{2}{*}{ 145: 4} & Upper $1 / 2$ & $\overline{6,849}$ & 6,550 & 6,699 \\
\hline S96T002928 & & Lower $1 / 2$ & 5,496 & 6,470 & $5,983^{\mathrm{oC} e \mathrm{e}}$ \\
\hline S96T002929 & \multirow[t]{2}{*}{$145: 5$} & Upper $\overline{1 / 2}$ & 4,403 & 4,620 & 4,511 \\
\hline S96T002930 & & Lower $1 / 2$ & 11,270 & 12,700 & $11,980^{\mathrm{QC}: \mathrm{e}}$ \\
\hline
\end{tabular}


Table B2-46. Tank 241-U-108 Core Sample Analytical Results: Phosphate (IC). (3 sheets)

\begin{tabular}{|c|c|c|c|c|c|}
\hline $\begin{array}{l}\text { Sample } \\
\text { Ninibr. }\end{array}$ & $\begin{array}{l}\text { Shingle. } \\
\text { I lacarton. }\end{array}$ & $\begin{array}{l}\text { Sample } \\
\text { Portion }\end{array}$ & Resul & Doulinere & Mera \\
\hline \multicolumn{2}{|c|}{ Sollths water ingest } & 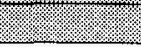 & .868 & 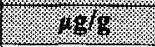 & $.186 \mathrm{c}$ \\
\hline S96T002931 & \multirow[t]{2}{*}{$145: 6$} & Upper $1 / 2$ & 13,720 & 14,500 & 14,110 \\
\hline S96T002932 & & Lower $1 / 2$ & 8,284 & 8,230 & $8, \overline{257}$ \\
\hline S96T002933 & \multirow[t]{2}{*}{$145: 7$} & Upper $1 / 2$ & 8,099 & 8,490 & 8,294 \\
\hline S96T002934 & & Lower $1 / 2$ & 5,737 & 7,130 & $6,43 \overline{3}^{\mathrm{QC}: \mathrm{e}}$ \\
\hline S96T002935 & \multirow[t]{2}{*}{$145: 8$} & Upper $1 / 2$ & 7,207 & 6,620 & 6,913 \\
\hline S96T002936 & & Lower $1 / 2$ & 7,026 & 8,680 & $7,853^{\text {कC:e }}$ \\
\hline S96T002937 & \multirow[t]{2}{*}{$145: 9$} & Upper $1 / 2$ & 39,760 & 40,100 & 39,930 \\
\hline S96T002938 & & Lower $1 / 2$ & $1.479 \mathrm{E}+05$ & $1.500 \mathrm{E}+05$ & $1.490 \mathrm{E}+05$ \\
\hline S96T003151 & 146: 1 & Upper $1 / 2$ & 5,552 & 7,960 & $6,756^{\mathrm{QC}: \mathrm{e}}$ \\
\hline S96T003152 & \multirow[t]{2}{*}{$146: 2$} & Upper $1 / 2$ & 6,977 & 7,770 & $7,373^{\mathrm{QC}: \mathrm{e}}$ \\
\hline S96T003153 & & Lower $1 / 2$ & 4,362 & 4,040 & 4,201 \\
\hline S96T003154 & \multirow[t]{2}{*}{$146: 3$} & Upper $1 / 2$ & 9,199 & 8,660 & 8,929 \\
\hline S96T003155 & & Lower $1 / 2$ & 2,098 & 1,770 & $1,934^{\mathrm{QC}: \mathrm{e}}$ \\
\hline S96T003052 & 146: $3 A$ & Upper $1 / 2$ & 4,261 & 4,300 & 4,280 \\
\hline S96T003053 & \multirow[t]{2}{*}{$146: 4$} & Upper $1 / 2$ & 7,488 & 7,770 & 7,629 \\
\hline S96T003054 & & Lower $1 / 2$ & 11,110 & 8,690 & $9,900^{\mathrm{QC}: \mathrm{e}}$ \\
\hline S96T003055 & \multirow[t]{2}{*}{ 146: 5} & Upper $1 / 2$ & 9,139 & 10,600 & $9,869^{\mathrm{oC}: \mathrm{e}}$ \\
\hline S96T003056 & & Lower $1 / 2$ & 7,657 & 7,360 & 7,508 \\
\hline S96T003057 & \multirow[t]{3}{*}{$146: 6$} & Upper $1 / 2$ & 10,770 & 9,400 & $10,090^{\mathrm{QC}: \mathrm{e}}$ \\
\hline S96T003058 & & Lower $1 / 2$ & 7,239 & 9,020 & $8,129^{\mathrm{QC:c}}$ \\
\hline S96T004322 & & Lower $1 / 2$ & 9,165 & 9,530 & 9,347 \\
\hline S96T003059 & \multirow[t]{2}{*}{$146: 7$} & Upper $1 / 2$ & 8,497 & 8,660 & 8,578 \\
\hline S96T003060 & & Lower $1 / 2$ & 15,290 & 15,600 & 15,440 \\
\hline S96T003156 & \multirow[t]{2}{*}{$146: 8$} & Upper $1 / 2$ & 6,367 & 6,380 & 6,373 \\
\hline S96T003157 & & Lower $1 / 2$ & 8,097 & 7,620 & 7,858 \\
\hline S96T004181 & \multirow[t]{2}{*}{$146: 9$} & Lower $1 / 2$ & $1.474 \mathrm{E}+05$ & $1.320 \mathrm{E}+05$ & $1.397 \mathrm{E}+05^{\mathrm{QC}: \mathrm{t}}$ \\
\hline S96T003158 & & Upper $1 / 2$ & 32,820 & 30,600 & $31,7 \overline{10}$ \\
\hline S96T003452 & Core 141 & $\begin{array}{l}\text { Solid } \\
\text { composite }\end{array}$ & 14,350 & 17,500 & $15,920^{\mathrm{eC}: \mathrm{e}}$ \\
\hline S96T003661 & Core 145 & $\begin{array}{l}\text { Solid } \\
\text { composite }\end{array}$ & 10,480 & 10,600 & 10,540 \\
\hline S96T004201 & Core 146 & $\begin{array}{l}\text { Solid } \\
\text { composite }\end{array}$ & 10,500 & 11,000 & 10,750 \\
\hline
\end{tabular}


Table B2-46. Tank 241-U-108 Core Sample Analytical Results: Phosphate (IC). (3 sheets)

\begin{tabular}{|c|c|c|c|c|c|}
\hline $\begin{array}{l}\text { Sample } \\
\text { Miminer }\end{array}$ & $\begin{array}{l}\text { Sample } \\
\text { L }\end{array}$ & $\begin{array}{l}\text { Samingle } \\
\text { Eortorn }\end{array}$ & Mesult & Dupplicate & Meari \\
\hline \multicolumn{2}{|c|}{ 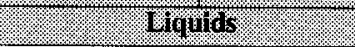 } & (2.: & $18 \mathrm{mi}$ & $.110 \mathrm{gmI}$ & Iglain \\
\hline S96T002282 & 141: 1 & $\begin{array}{l}\text { Drainable } \\
\text { liquid }\end{array}$ & 2,266 & 2,480 & 2,373 \\
\hline S96T002942 & 145: 1 & $\begin{array}{l}\text { Drainable } \\
\text { liquid }\end{array}$ & 2,690 & 2,690 & 2,690 \\
\hline S96T003163 & $146: 1$ & $\begin{array}{l}\text { Drainable } \\
\text { liquid }\end{array}$ & 2,529 & 2,470 & 2,499 \\
\hline
\end{tabular}

Table B2-47. Tank 241-U-108 Grab Sample Analytical Results: Phosphate (IC).

\begin{tabular}{|c|c|c|c|c|c|}
\hline $\begin{array}{l}\text { Sample } \\
\text { Vumber }\end{array}$ & $\begin{array}{l}\text { Sanviol } \\
\text { yocation }\end{array}$ & Sorioglo. & Resull & Buplate & Mean \\
\hline \multicolumn{2}{|c|}{ : Uquirs : } & & 4g/11. & Harits: & Holnis \\
\hline S95T000978 & Riser 7 & Grab sample & 3,700 & $<1,160$ & $<2,430^{\mathrm{eC}: \mathrm{e}}$ \\
\hline
\end{tabular}


Table B2-48. Tank 241-U-108 Core Sample Analytical Results: Sulfate (IC). (2 sheets)

\begin{tabular}{|c|c|c|c|c|c|}
\hline $\begin{array}{l}\text { Sanjols } \\
\text { Ninion }\end{array}$ & $\begin{array}{l}\text { Somple } \\
\text { Uraerano }\end{array}$ & $\begin{array}{l}\text { Sarmples } \\
\text { Yortion }\end{array}$ & Hesuil & Daphlantre & Mrean \\
\hline \multicolumn{2}{|c|}{ 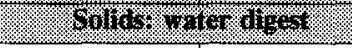 } & 3.12 & 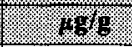 & 496 & $. / 4 \mathrm{~g} / \mathrm{s}$ \\
\hline S96T002270 & 141: 1 & Lower $1 / 2$ & 16,860 & 17,000 & 16,930 \\
\hline S96T002273 & $141: 2$ & Upper $1 / 2$ & 13,550 & 15,900 & $14,720^{\mathrm{QC: \textrm {e }}}$ \\
\hline S96T002274 & $141: 3$ & Upper $1 / 2$ & 11,290 & $\overline{10,600}$ & 10,940 \\
\hline S96T002275 & 141: $3 \mathrm{~A}$ & Upper $1 / 2$ & 10,860 & 9,000 & $9,930^{\mathrm{QC:e}}$ \\
\hline S96T002276 & \multirow[t]{2}{*}{ 141: 4} & Upper $1 / 2$ & 18,860 & 18,100 & 18,480 \\
\hline S96T002277 & & Lower $1 / 2$ & 14,310 & 14,700 & 14,500 \\
\hline S96T002278 & \multirow[t]{2}{*}{ 141: $4 \mathrm{~A}$} & Upper $1 / 2$ & 14,220 & 12,900 & 13,560 \\
\hline S96T002279 & & Lower $1 / 2$ & 15,650 & 16,600 & 16,120 \\
\hline S96T002617 & \multirow[t]{2}{*}{$141: 5$} & Upper $1 / 2$ & 12,070 & 12,300 & 12,180 \\
\hline S96T002618 & & Lower $1 / 2$ & 9,603 & 9,550 & 9,576 \\
\hline S96T002619 & \multirow[t]{2}{*}{$141: 6$} & Upper $1 / 2$ & 12,600 & 12,900 & 12,750 \\
\hline S96T002620 & & Lower $1 / 2$ & 10,960 & 10,500 & 10,730 \\
\hline S96T002621 & \multirow[t]{2}{*}{ 141: 7} & Upper $1 / 2$ & 20,100 & 20,100 & 20,100 \\
\hline S96T002622 & & Lower $1 / 2$ & 17,940 & 17,400 & 17,670 \\
\hline S96T002623 & \multirow[t]{2}{*}{$141: 8$} & Upper $1 / 2$ & 21,420 & 19,700 & 20,560 \\
\hline S96T002624 & & Lower $1 / 2$ & $<1,278$ & $<1,290$ & $<1,284$ \\
\hline S96T002625 & 141: 9 & Upper $1 / 2$ & 28,440 & 27,500 & 27,970 \\
\hline S96T002924 & $145: 2$ & Upper $1 / 2$ & 6,813 & 6,560 & 6,686 \\
\hline S96T002925 & \multirow[t]{2}{*}{$145: 3$} & Upper $1 / 2$ & 10,670 & 10,200 & 10,430 \\
\hline S96T002926 & & Lower $1 / 2$ & 12,570 & 13,900 & $13,230^{\mathrm{QC:e}}$ \\
\hline S96T002927 & \multirow[t]{2}{*}{ 145: 4} & Upper $1 / 2$ & 8,652 & 7,950 & 8,301 \\
\hline S96T002928 & & Lower $1 / 2$ & 11,430 & 11,900 & 11,660 \\
\hline S96T002929 & \multirow[t]{2}{*}{$145: 5$} & Upper $1 / 2$ & 15,390 & 15,900 & 15,640 \\
\hline S96T002930 & & Lower $1 / 2$ & 13,720 & 14,600 & $14, \overline{160}$ \\
\hline S96T002931 & \multirow[t]{2}{*}{$145: 6$} & Upper $1 / 2$ & 15,560 & 16,700 & 16,130 \\
\hline S96T002932 & & Lower $1 / 2$ & 16,240 & 16,600 & 16,420 \\
\hline S96T002933 & \multirow[t]{2}{*}{$145: 7$} & Upper $1 / 2$ & 17,880 & 18,000 & 17,940 \\
\hline S96T002934 & & Lower $1 / 2$ & 12,500 & 16,000 & $14,250^{\mathrm{QC: \textrm {e }}}$ \\
\hline S96T002935 & \multirow[t]{2}{*}{$145: 8$} & Upper $1 / 2$ & 12,440 & 12,800 & 12,620 \\
\hline S96T002936 & & Lower $1 / 2$ & 28,540 & 26,900 & 27,720 \\
\hline S96T002937 & \multirow[t]{2}{*}{$145: 9$} & Upper $1 / 2$ & 29,590 & 21,200 & $25,390^{\mathrm{QC:e}}$ \\
\hline S96T002938 & & Lower $1 / 2$ & 1,917 & 1,620 & $1,768^{\mathrm{QC}: \mathrm{e}}$ \\
\hline
\end{tabular}


Table B2-48. Tank 241-U-108 Core Sample Analytical Results: Sulfate (IC). (2 sheets)

\begin{tabular}{|c|c|c|c|c|c|}
\hline $\begin{array}{l}\text { Somplo } \\
\text { Juminer }\end{array}$ & 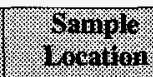 & $\begin{array}{l}\text { Saraple: } \\
\text { rotion. }\end{array}$ & Res.111 & 8uplicate & Nean \\
\hline \multicolumn{2}{|c|}{ 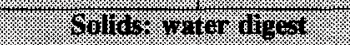 } & 1.: & .400 & 4.496 & 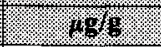 \\
\hline S96T003151 & 146: 1 & Upper $1 / 2$ & 22,100 & 22,600 & 22,350 \\
\hline S96T003152 & \multirow[t]{2}{*}{ 146: 2} & Upper $1 / 2$ & 15,850 & 16,800 & 16,320 \\
\hline S96T003153 & & Lower $1 / 2$ & 3,853 & 3,860 & $3,8 \overline{56}$ \\
\hline S96T003154 & \multirow[t]{2}{*}{$146: 3$} & Upper $1 / 2$ & $23, \overline{870}$ & $\overline{24}, 200$ & 24,030 \\
\hline S96T003155 & & Lower $1 / 2$ & $<1,750$ & 1,990 & $<1,870^{\mathrm{QC}: \mathrm{e}}$ \\
\hline S96T003052 & 146: $3 \mathrm{~A}$ & Upper $1 / 2$ & 12,230 & 12,300 & 12,260 \\
\hline S96T003053 & \multirow[t]{2}{*}{ 146: 4} & Upper $1 / 2$ & 9,000 & $9, \overline{070}$ & 9,035 \\
\hline S96T003054 & & Lower $1 / 2$ & 15,640 & 17,100 & 16,370 \\
\hline S96T003055 & \multirow[t]{2}{*}{$146: 5$} & Upper $1 / 2$ & 18,280 & 18,600 & 18,440 \\
\hline S96T003056 & & Lower $1 / 2$ & 13,580 & 13,500 & 13,540 \\
\hline S96T003057 & \multirow[t]{3}{*}{ 146: 6} & Upper $1 / 2$ & 16,240 & 16,200 & 16,220 \\
\hline \$96T003058 & & Lower $1 / 2$ & 13,640 & 18,300 & $15,970^{\mathrm{QC}: \mathrm{e}}$ \\
\hline S96T004322 & & Lower $1 / 2$ & 19,210 & 19,100 & 19,150 \\
\hline S96T003059 & \multirow[t]{2}{*}{ 146: 7} & Upper $1 / 2$ & 17,380 & 17,400 & 17,390 \\
\hline S96T003060 & & Lower $1 / 2$ & 20,080 & 20,000 & 20,040 \\
\hline S96T003156 & \multirow[t]{2}{*}{$146: 8$} & Upper $1 / 2$ & 19,420 & 19,400 & 19,410 \\
\hline S96T003157 & & Lower $1 / 2$ & 31,980 & 31,000 & 31,490 \\
\hline S96T004181 & \multirow[t]{2}{*}{ 146: 9} & Lower $1 / 2$ & 1,125 & 996 & $1,060^{\mathrm{QC}: \mathrm{e}}$ \\
\hline S96T003158 & & Upper $1 / 2$ & 33,110 & 33,700 & 33,450 \\
\hline S96T003452 & Core 141 & $\begin{array}{l}\text { Solid } \\
\text { composite }\end{array}$ & 17,290 & 16,900 & 17,090 \\
\hline S96T003661 & Core 145 & $\begin{array}{l}\text { Solid } \\
\text { composite }\end{array}$ & 17,940 & 17,000 & 17,470 \\
\hline S96T004201 & Core 146 & $\begin{array}{l}\text { Solid } \\
\text { composite }\end{array}$ & $18, \overline{490}$ & 17,900 & 18,190 \\
\hline \multicolumn{2}{|c|}{ Whowids: } & (3) & (nglint & 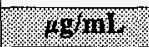 & ogmil. \\
\hline S96T002282 & 141: 1 & $\begin{array}{l}\text { Drainable } \\
\text { liquid }\end{array}$ & 3,058 & 2,920 & 2,989 \\
\hline S96T002942 & $145: 1$ & $\begin{array}{l}\text { Drainable } \\
\text { liquid }\end{array}$ & 2,958 & 2,950 & 2,954 \\
\hline S96T003163 & $146: 1$ & $\begin{array}{l}\text { Drainable } \\
\text { liquid }\end{array}$ & 3,630 & 4,320 & $3,975^{\mathrm{QC}: \mathrm{e}}$ \\
\hline
\end{tabular}


Table B2-49. Tank 241-U-108 Grab Sample Analytical Results: Sulfate (IC).

\begin{tabular}{|c|c|c|c|c|c|}
\hline $\begin{array}{l}\text { Sminglo. } \\
\text { funiser. }\end{array}$ & Shanglo & $\begin{array}{l}\text { Sample } \\
\text { Portion. }\end{array}$ & Resuil & Duplicare & Wean \\
\hline \multicolumn{2}{|c|}{ Irquals : } & & 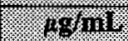 & 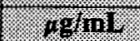 & $1.96111 \%$ \\
\hline S95T000978 & Riser 7 & Grab sample & 4,390 & 4,800 & 4,595 \\
\hline
\end{tabular}

Table B2-50. Tank 241-U-108 Core Sample Analytical Results: Oxalate (IC). (3 sheets)

\begin{tabular}{|c|c|c|c|c|c|}
\hline $\begin{array}{l}\text { Sample: } \\
\text { Trumber. }\end{array}$ & 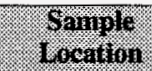 & $\begin{array}{l}\text { Sivingle } \\
\text { rortion }\end{array}$ & nesult & Duplicate & Mean: \\
\hline \multicolumn{2}{|c|}{ 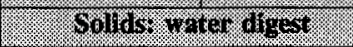 } & থ. & .894. & 1998 & 1088 \\
\hline S96T002270 & 141: 1 & Lower $1 / 2$ & 12,480 & 12,600 & 12,540 \\
\hline S96T002273 & $141: 2$ & Upper $1 / 2$ & 3,090 & 4,310 & $3,700^{\alpha \mathrm{C}} \mathrm{e}$ \\
\hline S96'T002274 & 141: 3 & Upper $1 / 2$ & 3,014 & 3,160 & 3,087 \\
\hline S96T002275 & $141: 3 \mathrm{~A}$ & Upper $1 / 2$ & 1,364 & 1,410 & 1,387 \\
\hline S96T002276 & \multirow[t]{2}{*}{ 141: 4} & Upper $1 / 2$ & 3,245 & 2,950 & 3,097 \\
\hline S96T002277 & & Lower $1 / 2$ & 3,155 & 3,790 & $3,472^{\overline{\mathrm{QC}: e}}$ \\
\hline S96T002278 & \multirow[t]{2}{*}{ 141: $4 \mathrm{~A}$} & Upper $1 / 2$ & 3,086 & 2,880 & 2,983 \\
\hline S96T002279 & & Lower $1 / 2$ & 2,887 & 3,200 & $\overline{3,043^{\mathrm{QC}: \mathrm{e}}}$ \\
\hline S96T002617 & \multirow[t]{2}{*}{$141: 5$} & Upper $1 / 2$ & $3, \overline{474}$ & 3,380 & 3,427 \\
\hline S96T002618 & & Lower $1 / 2$ & $2, \overline{931}$ & $2, \overline{750}$ & 2,840 \\
\hline S96T002619 & \multirow[t]{2}{*}{ 141: 6} & Upper $1 / 2$ & $<934$ & 2,670 & $<1,802^{\mathrm{QC}: \mathrm{e}}$ \\
\hline S96T002620 & & Lower $1 / 2$ & 2,655 & 2,840 & 2,747 \\
\hline S96T002621 & \multirow[t]{2}{*}{ 141: 7} & Upper $1 / 2$ & 5,043 & 5,360 & 5,201 \\
\hline S96T002622 & & Lower $1 / 2$ & 4,085 & 3,410 & $3,747^{\mathrm{QC}: e}$ \\
\hline S96T002623 & \multirow[t]{2}{*}{ 141: 8} & Upper $1 / 2$ & 4,764 & 4,520 & 4,642 \\
\hline S96T002624 & & Lower $1 / 2$ & 4,248 & $3, \overline{440}$ & $3,844^{\mathrm{QC} e \mathrm{e}}$ \\
\hline S96T002625 & $141: 9$ & Upper $1 / 2$ & 8,378 & 7,950 & 8,164 \\
\hline S96T002924 & 145: 2 & Upper $1 / 2$ & 2,270 & 2,420 & 2,345 \\
\hline S96T002925 & \multirow[t]{2}{*}{$145: 3$} & Upper $1 / 2$ & 2,984 & 2,950 & 2,967 \\
\hline S96T002926 & & Lower $1 / 2$ & 2,361 & 2,670 & $2,515^{Q C: e}$ \\
\hline S96T002927 & \multirow[t]{2}{*}{$145: 4$} & Upper $1 / 2$ & 2,696 & 2,030 & $2,363^{\mathrm{QC}: \mathrm{\epsilon}}$ \\
\hline S96T002928 & & Lower $1 / 2$ & 2,933 & 3,250 & $3,091^{\mathrm{QC}: \mathrm{e}}$ \\
\hline S96T002929 & \multirow[t]{2}{*}{$145: 5$} & Upper $1 / 2$ & 3,238 & $3,4 \overline{70}$ & 3,354 \\
\hline S96T002930 & & Lower $1 / 2$ & 5,302 & 5,410 & 5,356 \\
\hline
\end{tabular}


Table B2-50. Tank 241-U-108 Core Sample Analytical Results: Oxalate (IC). (3 sheets)

\begin{tabular}{|c|c|c|c|c|c|}
\hline $\begin{array}{l}\text { Sinule } \\
\text { Nomber }\end{array}$ & $\begin{array}{l}\text { Garivilo. } \\
\text { iscation }\end{array}$ & $\begin{array}{l}\text { Sarmple: } \\
\text { Portion. }\end{array}$ & Result & Duplicate: & Irean \\
\hline \multicolumn{2}{|c|}{ Solkits water digets } & & .856 & $199 / 8$ & $135 \mathrm{~g}$ \\
\hline S96T002931 & \multirow[t]{2}{*}{ 145: 6} & Upper $1 / 2$ & 4,547 & 4,770 & 4,658 \\
\hline S96T002932 & & Lower $1 / 2$ & 3,645 & 3,740 & 3,692 \\
\hline S96T002933 & \multirow[t]{2}{*}{$145: 7$} & Upper $1 / 2$ & 4,068 & 3,960 & 4,014 \\
\hline S96T002934 & & Lower $1 / 2$ & 3,363 & 4,400 & $3,881^{\mathrm{QC}: e}$ \\
\hline S96T002935 & \multirow[t]{2}{*}{$145: 8$} & Upper $1 / 2$ & 2,585 & 2,870 & $2,727^{\mathrm{QC}: \mathrm{e}}$ \\
\hline S96T002936 & & Lower $1 / 2$ & 4,737 & $\overline{4,590}$ & $\overline{4,663}$ \\
\hline S96T002937 & \multirow[t]{2}{*}{$145: 9$} & Upper $1 / 2$ & 18,440 & $\overline{12,700}$ & $15,570^{\mathrm{QC}: e}$ \\
\hline S96T002938 & & Lower $1 / 2$ & 2,747 & 2,780 & 2,763 \\
\hline S96T003151 & 146: 1 & Upper $1 / 2$ & 2,595 & 2,830 & 2,712 \\
\hline S96T003152 & \multirow[t]{2}{*}{ 146: 2} & Upper $1 / 2$ & 2,179 & $2, \overline{630}$ & $2,404^{\mathrm{QC}: \mathrm{e}}$ \\
\hline S96T003153 & & Lower $1 / 2$ & $<2,025$ & $<2,230$ & $<2,127$ \\
\hline S96T003154 & \multirow[t]{2}{*}{$146: 3$} & Upper $1 / 2$ & 2,913 & 2,930 & 2,921 \\
\hline S96T003155 & & Lower $1 / 2$ & $<1,352$ & $<1,470$ & $<1,411$ \\
\hline S96T003052 & 146: $3 A$ & Upper $1 / 2$ & 1,850 & 2,130 & $1,990^{\mathrm{QC:e}}$ \\
\hline S96T003053 & \multirow[t]{2}{*}{ 146: 4} & Upper $1 / 2$ & 2,532 & 2,670 & 2,601 \\
\hline S96T003054 & & Lower $1 / 2$ & 4,230 & 4,210 & 4,220 \\
\hline S96T003055 & \multirow[t]{2}{*}{$146: 5$} & Upper $1 / 2$ & 4,588 & 4,600 & 4,594 \\
\hline S96T003056 & & Lower $1 / 2$ & 3,917 & 3,790 & 3,853 \\
\hline S96T003057 & \multirow[t]{3}{*}{$146: 6$} & Upper $1 / 2$ & 3,905 & 4,450 & $4,177^{\mathrm{QC}: \mathrm{e}}$ \\
\hline S96T003058 & & Lower $1 / 2$ & 2,944 & 3,960 & $3,452^{\mathrm{QC}: \mathrm{e}}$ \\
\hline S96T004322 & & Lower $1 / 2$ & 4,208 & 3,750 & $3,979^{\mathrm{QC}: \mathrm{e}}$ \\
\hline S96T003059 & \multirow[t]{2}{*}{$146: 7$} & Upper $1 / 2$ & 3,717 & 3,960 & 3,838 \\
\hline S96T003060 & & Lower $1 / 2$ & 5,001 & 5,190 & 5,095 \\
\hline S96T003156 & \multirow[t]{2}{*}{ 146: 8} & Upper $1 / 2$ & $4, \overline{478}$ & 4,470 & 4,474 \\
\hline S96T003157 & & Lower $1 / 2$ & 6,418 & 6,320 & 6,369 \\
\hline S96T004181 & \multirow[t]{2}{*}{$146: 9$} & Lower $1 / 2$ & 6,532 & 6,740 & 6,636 \\
\hline S96T003158 & & Upper $1 / 2$ & 20,100 & 20,600 & $20, \overline{350}$ \\
\hline S96T003452 & Core 141 & $\begin{array}{l}\text { Solid } \\
\text { composite }\end{array}$ & 4,625 & 4,520 & $4,5 \overline{72}$ \\
\hline S96T003661 & Core 145 & $\begin{array}{l}\text { Solid } \\
\text { composite }\end{array}$ & 4,900 & 5,010 & 4,955 \\
\hline S96T004201 & Core 146 & $\begin{array}{l}\text { Solid } \\
\text { composite }\end{array}$ & 4,407 & 4,350 & 4,378 \\
\hline
\end{tabular}


Table B2-50. Tank 241-U-108 Core Sample Analytical Results: Oxalate (IC). (3 sheets)

\begin{tabular}{|c|c|c|c|c|c|}
\hline Squnglo & $\begin{array}{l}\text { Gamplo } \\
\text { yocation }\end{array}$ & $\begin{array}{l}\text { Gumple } \\
\text { Pongion }\end{array}$ & Resul & Duplicate & Mean \\
\hline \multicolumn{2}{|c|}{ 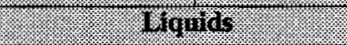 } & 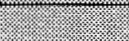 & 094111 & $10 \mathrm{mu}$ & $8 \mathrm{~min}$ \\
\hline S96T002282 & 141: 1 & $\begin{array}{l}\text { Drainable } \\
\text { liquid }\end{array}$ & $<434.8$ & $<435$ & $<434.9$ \\
\hline S96T002942 & $145: 1$ & $\begin{array}{l}\text { Drainable } \\
\text { liquid }\end{array}$ & $<434.8$ & 736 & $<585.4^{\mathrm{QC:e}}$ \\
\hline S96T003163 & 146: 1 & $\begin{array}{l}\text { Drainable } \\
\text { liquid }\end{array}$ & $<540.9$ & $<541$ & $<540.95$ \\
\hline
\end{tabular}

\section{B2.5 RADIOCHEMICAL ANALYSES}

\section{B2.5.1 Total Alpha Activity}

Analyses for total alpha activity were performed on core samples recovered from tank 241-U-108. The samples were prepared by potassium hydroxide fusion digestion according to procedure LA-549-141, Rev. F-0 and analyzed according to procedure LA-508-101, Rev. D-2. Two fusions were prepared per sample (for duplicate results). Each fused dilution was analyzed twice, the results were averaged and reported as one value. The highest result returned was $0.364 \mu \mathrm{Ci} / \mathrm{g}$. The sample results for total alpha are given in Table B2-51.

Table B2-51. Tank 241-U-108 Core Sample Analytical Results: Total Alpha. (3 sheets)

\begin{tabular}{|c|c|c|c|c|c|}
\hline 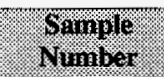 & $\begin{array}{l}\text { Sorinle } \\
\text { Yoration }\end{array}$ & $\begin{array}{l}\text { Simple: } \\
\text { Portion }\end{array}$ & Resuit & Duplicate & Yean \\
\hline \multicolumn{2}{|c|}{ 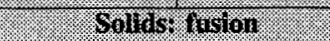 } & 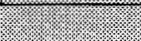 & 10.68 & is 0019 & 1018 \\
\hline S96T002257 & 141: 1 & Lower $1 / 2$ & 0.0932 & 0.0669 & $0.08005^{Q C:}$ \\
\hline S96T002258 & 141: 2 & Upper $1 / 2$ & 0.0352 & 0.0282 & $0.0317^{\mathrm{QC}: \mathrm{e}}$ \\
\hline S96T002259 & $141: 3$ & Upper $1 / 2$ & 0.0276 & 0.0313 & $0.02945^{\mathrm{QC}: \mathrm{e}}$ \\
\hline S96T002260 & 141: $3 \mathrm{~A}$ & Upper $1 / 2$ & 0.029 & 0.0232 & $0.0261^{\overline{Q C i e, \Gamma}}$ \\
\hline S96T002261 & \multirow[t]{2}{*}{ 141: 4} & Upper $1 / 2$ & 0.056 & 0.0456 & $0.0508^{\mathrm{QC}: e, \mathrm{f}}$ \\
\hline S96T002262 & & Lower $1 / 2$ & 0.0433 & 0.0374 & $0.04035^{0 C: e, f}$ \\
\hline S96T002263 & \multirow[t]{2}{*}{ 141: 4A } & Upper $1 / 2$ & 0.0538 & 0.0528 & 0.0533 \\
\hline S96T002264 & & Lower $1 / 2$ & 0.0291 & 0.0327 & $0.0309^{Q \mathrm{C}: e}$ \\
\hline S96T002608 & \multirow[t]{2}{*}{$141: 5$} & Upper $1 / 2$ & 0.0521 & 0.0604 & $0.05625^{\mathrm{QC}: \mathrm{e}}$ \\
\hline S96T002609 & & Lower $1 / 2$ & 0.0505 & 0.0452 & $0.04785^{\mathrm{QC}:}$ \\
\hline
\end{tabular}


Table B2-51. Tank 241-U-108 Core Sample Analytical Results: Total Alpha. (3 sheets)

\begin{tabular}{|c|c|c|c|c|c|}
\hline $\begin{array}{l}\text { Gonple } \\
\text { Ninnigrs. }\end{array}$ & Sorouition & $\begin{array}{l}\text { Sample } \\
\text { rortion }\end{array}$ & resailin & Bupliente & Mean \\
\hline \multicolumn{2}{|c|}{ Solids rusins : } & ?. & 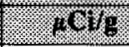 & .919. & 11019 \\
\hline S96T002610 & \multirow[t]{2}{*}{$141: 6$} & Upper $1 / 2$ & 0.0365 & 0.0279 & $0.0322^{\mathrm{QC}: \mathrm{e}}$ \\
\hline S96T002611 & & Lower $1 / 2$ & 0.0123 & $0.01 \overline{98}$ & $0.01605^{\overline{\mathrm{CC}: e}}$ \\
\hline S96T002612 & \multirow[t]{2}{*}{$141: 7$} & Upper $1 / 2$ & 0.0314 & 0.0383 & $0.03485^{\mathrm{QC:b,e}}$ \\
\hline S96T002613 & & Lower $1 / 2$ & 0.0296 & 0.0289 & $0.02925^{\mathrm{QC:b}}$ \\
\hline S96T002614 & \multirow[t]{2}{*}{$141: 8$} & Upper $1 / 2$ & 0.0714 & $<0.00429$ & $<0.03784^{\mathrm{QC:b}}$ \\
\hline S96T002615 & & Lower $1 / 2$ & 0.109 & 0.11 & $0.1095^{\overline{\mathrm{QC}: b}}$ \\
\hline S96T002616 & 141: 9 & Upper $1 / 2$ & 0.0486 & 0.0368 & $0.0427^{Q C: b, e}$ \\
\hline S96T002909 & $145: 2$ & Upper $1 / 2$ & 0.0269 & 0.0272 & 0.02705 \\
\hline S96T002910 & \multirow[t]{2}{*}{$145: 3$} & Upper $1 / 2$ & 0.0407 & 0.0423 & 0.0415 \\
\hline S96T002911 & & Lower $1 / 2$ & $0 . \overline{423}$ & 0.0369 & $0.0396^{\overline{\mathrm{QC}: e}}$ \\
\hline S96T002912 & \multirow[t]{2}{*}{$145: 4$} & Upper 1/2 & 0.0352 & 0.0317 & $0.03345^{\mathrm{QC:a,e,f}}$ \\
\hline S96T002913 & & Lower $1 / 2$ & 0.0464 & 0.0472 & $0.0468^{\mathrm{eC}: \mathrm{a}, \mathrm{f}}$ \\
\hline S96T002914 & \multirow[t]{2}{*}{$145: 5$} & Upper $1 / 2$ & 0.0353 & 0.0396 & $0.03745^{\overline{\mathrm{QC:a}, \mathrm{e}, \overline{\mathrm{f}}}}$ \\
\hline S96T002915 & & Lower $1 / 2$ & 0.0302 & 0.0329 & 0.03155 \\
\hline S96T002916 & \multirow[t]{2}{*}{$145: 6$} & Upper $1 / 2$ & 0.0195 & $0 . \overline{0145}$ & $0.017^{\mathrm{QC}: \mathrm{e}}$ \\
\hline S96T002917 & & Lower $1 / 2$ & 0.0151 & 0.0135 & $0.0143^{\mathrm{QCe}}$ \\
\hline S96T002918 & \multirow[t]{2}{*}{$145: 7$} & Upper $1 / 2$ & 0.0158 & 0.0177 & $0.01675^{\text {QC:b,e }}$ \\
\hline S96T002919 & & Lower $1 / 2$ & 0.0213 & 0.0214 & $0.02135^{\mathrm{QC}: \mathrm{b}}$ \\
\hline 596T002920 & \multirow[t]{2}{*}{$145: 8$} & Upper $1 / 2$ & 0.0126 & 0.0127 & $0.01265^{\mathrm{QC}: \mathrm{b}}$ \\
\hline S96T002921 & & Lower $1 / 2$ & 0.0313 & 0.0308 & $0.03105^{Q \mathrm{QC:} \mathrm{b}}$ \\
\hline S96T002922 & \multirow[t]{2}{*}{$145: 9$} & Upper $1 / 2$ & 0.262 & 0.245 & $0.2535^{\overline{Q C: b}}$ \\
\hline S96T002923 & & Lower $1 / 2$ & 0.0718 & 0.055 & $0.0634^{\mathrm{QC:b}, \mathrm{e}}$ \\
\hline S96T003143 & 146: 1 & Upper $1 / 2$ & 0.0387 & 0.0394 & $0.03905^{\mathrm{QC:s}}$ \\
\hline S96T003144 & \multirow[t]{2}{*}{$146: 2$} & Upper $1 / 2$ & 0.0255 & 0.0274 & $0.02645^{\mathrm{OC:a}}$ \\
\hline S96T003145 & & Lower $1 / 2$ & 0.0129 & 0.0117 & $0.0123^{\mathrm{QC}: \mathrm{a}}$ \\
\hline S96T003146 & \multirow[t]{2}{*}{$146: 3$} & Upper $1 / 2$ & 0.033 & 0.0332 & 0.0331 \\
\hline S96T003147 & & Lower $1 / 2$ & 0.0104 & 0.0111 & 0.01075 \\
\hline S96T003001 & 146: $3 \mathrm{~A}$ & Upper $1 / 2$ & 0.0289 & 0.0285 & 0.0287 \\
\hline S96T003002 & \multirow[t]{2}{*}{ 146: 4} & Upper $1 / 2$ & $0 . \overline{0396}$ & 0.0388 & $0.0392^{\mathrm{QC:b}}$ \\
\hline S96T003003 & & Lower $1 / 2$ & 0.112 & 0.0913 & $0.10165^{\text {QC:b,e }}$ \\
\hline S96T003004 & \multirow[t]{2}{*}{$146: 5$} & Upper $1 / 2$ & 0.0816 & 0.0975 & $0.08955^{\mathrm{QC}: \mathrm{b}, \mathrm{e}}$ \\
\hline S96T003005 & & Lower $1 / 2$ & 0.0461 & $<0.0602$ & $<0.05315^{\mathrm{Qce}}$ \\
\hline
\end{tabular}


Table B2-51. Tank 241-U-108 Core Sample Analytical Results: Total Alpha. (3 sheets)

\begin{tabular}{|c|c|c|c|c|c|}
\hline S1m & oocatisn & 301010 & Masili: & Bujlloute & rotern \\
\hline \multicolumn{2}{|c|}{ 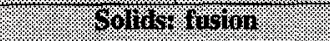 } & (3) & (z) & 1018 & (1) \\
\hline S96T003006 & \multirow[t]{2}{*}{ 146: 6} & Upper $1 / 2$ & $<0.0324$ & $<0.0581$ & $<0.04525^{Q C: e}$ \\
\hline \$96T003007 & & Lower $1 / 2$ & $<0.0925$ & $<0.126$ & $<0.10925^{\mathrm{QC}: \mathrm{e}}$ \\
\hline S96T003008 & \multirow[t]{2}{*}{$146: 7$} & Upper $1 / 2$ & 0.0178 & 0.0196 & 0.0187 \\
\hline \$96T003009 & & Lower $1 / 2$ & 0.0307 & 0.0249 & $0.0278^{Q C: e}$ \\
\hline S96T003148 & \multirow[t]{2}{*}{ 146: 8} & Upper $1 / 2$ & 0.0223 & 0.026 & $0.02415^{\mathrm{QC}: \mathrm{e}}$ \\
\hline S96T003149 & & Lower $1 / 2$ & 0.0389 & 0.0514 & $0.04515^{\mathrm{QC:e}}$ \\
\hline S96T004180 & \multirow[t]{2}{*}{ 146: 9} & Lower $1 / 2$ & 0.363 & 0.365 & 0.364 \\
\hline S96T003150 & & Upper $1 / 2$ & 0.182 & 0.171 & 0.1765 \\
\hline S96T003450 & Core 141 & $\begin{array}{l}\text { Solid } \\
\text { composite }\end{array}$ & 0.0312 & 0.0359 & $0.03355^{\mathrm{QC}: \mathrm{a}, \mathrm{e}}$ \\
\hline S96T003660 & Core 145 & $\begin{array}{l}\text { Solid } \\
\text { composite }\end{array}$ & 0.0549 & 0.0338 & $0.04435^{\overline{\mathrm{QC} ; \mathrm{a}, \mathrm{e}}}$ \\
\hline S96T004199 & Core 146 & $\begin{array}{l}\text { Solid } \\
\text { composite }\end{array}$ & 0.0539 & $0 . \overline{0541}$ & $0.054^{\mathrm{QC}: \mathrm{f}}$ \\
\hline
\end{tabular}

\section{B2.5.2 Strontium-90}

The activity of Sr-90 was determined on solid core samples by chemical separation of fusion digests followed by beta counting according to procedure LA-220-101, Rev. D-1. Analysis for the 1995 grab sample was by beta counting following chemical separation according to procedure LA-220-101, Rev. D-1. Activities of Sr-90 ranged from $0.345 \mu \mathrm{Ci} / \mathrm{g}$ (grab sample U-108-1) to $64.5 \mu \mathrm{Ci} / \mathrm{g}$ (upper half of segment 9, core 145). All results are shown in Tables B2-52 and B2-53. 
Table B2-52. Tank 241-U-108 Core Sample Analytical Results: Strontium-89/90. ( 2 sheets)

\begin{tabular}{|c|c|c|c|c|c|}
\hline $\begin{array}{l}\text { Gomple. } \\
\text { Nomilor }\end{array}$ & Soringle & Sample & Pesint & Buplicate & Mean \\
\hline \multicolumn{3}{|c|}{ 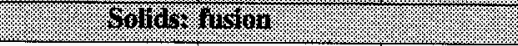 } & 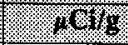 & 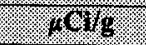 & 1006 \\
\hline S96T002257 & 141: 1 & Lower $1 / 2$ & 15 & 10.9 & 12.95 \\
\hline S96T002258 & 141: 2 & Upper $1 / 2$ & 5.96 & 6.42 & 6.19 \\
\hline S96T002259 & 141: 3 & Upper $1 / 2$ & 5.5 & 5.67 & 5.585 \\
\hline S96T002260 & 141: $3 \mathrm{~A}$ & Upper $1 / 2$ & 3.79 & 4.03 & 3.91 \\
\hline S96T002261 & \multirow[t]{2}{*}{ 141: 4} & Upper $1 / 2$ & 8.12 & 7.8 & 7.96 \\
\hline S96T002262 & & Lower $1 / 2$ & 6.84 & 7.66 & 7.25 \\
\hline S96T002263 & \multirow[t]{2}{*}{$141: 4 \mathrm{~A}$} & Upper $1 / 2$ & 7.19 & 7.19 & 7.19 \\
\hline S96T002264 & & Lower $1 / 2$ & 5.68 & 5.67 & 5.675 \\
\hline S96T002608 & \multirow[t]{2}{*}{$141: 5$} & Upper $1 / 2$ & 6.29 & 6.49 & 6.39 \\
\hline S96T002609 & & Lower $1 / 2$ & 5.16 & 5.08 & 5.12 \\
\hline S96T002610 & \multirow[t]{2}{*}{ 141: 6} & Upper $1 / 2$ & 4.92 & 4.92 & 4.92 \\
\hline S96T002611 & & Lower $1 / 2$ & 4.22 & 4.41 & 4.315 \\
\hline S96T002612 & \multirow{2}{*}{$141: 7$} & Upper $1 / 2$ & 10.2 & 10.6 & 10.4 \\
\hline S96T002613 & & Lower $1 / 2$ & 9.6 & 8.95 & 9.275 \\
\hline S96T002614 & \multirow[t]{2}{*}{ 141: 8} & Upper $1 / 2$ & 12.6 & 12.1 & 12.35 \\
\hline S96T002615 & & Lower $1 / 2$ & 21.2 & 21.1 & 21.15 \\
\hline S96T002616 & 141: 9 & Upper $1 / 2$ & 3.9 & 3.29 & 3.595 \\
\hline S96T002909 & $145: 2$ & Upper $1 / 2$ & 3.19 & 4.08 & 3.635 \\
\hline S96T002910 & \multirow[t]{2}{*}{$145: 3$} & Upper $1 / 2$ & 6.01 & 5.05 & 5.53 \\
\hline S96T002911 & & Lower $1 / 2$ & 5.22 & 4.98 & 5.10 \\
\hline S96T002912 & \multirow[t]{2}{*}{$145: 4$} & Upper $1 / 2$ & 4.31 & 4.42 & 4.365 \\
\hline S96T002913 & & Lower $1 / 2$ & 6.26 & 5.99 & 6.125 \\
\hline S96T002914 & \multirow[t]{2}{*}{$145: 5$} & Upper $1 / 2$ & 6.08 & 6.5 & 6.29 \\
\hline S96T002915 & & Lower $1 / 2$ & 11.5 & 11.4 & 11.45 \\
\hline S96T002916 & \multirow[t]{2}{*}{$145: 6$} & Upper $1 / 2$ & 9.25 & 8.83 & 9.04 \\
\hline S96T002917 & & Lower $1 / 2$ & 7.05 & 7.45 & 7.25 \\
\hline S96T002918 & \multirow[t]{2}{*}{$145: 7$} & Upper $1 / 2$ & 8.25 & 8.59 & 8.42 \\
\hline S96T002919 & & Lower $1 / 2$ & 10 & 10.1 & 10.05 \\
\hline S96T002920 & \multirow[t]{2}{*}{$145: 8$} & \begin{tabular}{|l|} 
Upper $1 / 2$ \\
\end{tabular} & 5.92 & 5.7 & 5.81 \\
\hline S96T002921 & & Lower $1 / 2$ & 13.2 & 12.7 & 12.95 \\
\hline S96T002922 & \multirow[t]{2}{*}{$145: 9$} & Upper $1 / 2$ & 63.1 & 65.9 & 64.5 \\
\hline S96T002923 & & Lower $1 / 2$ & 11.4 & 11.9 & 11.65 \\
\hline
\end{tabular}


Table B2-52. Tank 241-U-108 Core Sample Analytical Results: Strontium-89/90. (2 sheets)

\begin{tabular}{|c|c|c|c|c|c|}
\hline $\begin{array}{l}\text { Sumile } \\
\text { Yuniver }\end{array}$ & I Socition & $\begin{array}{l}\text { Sample } \\
\text { Poction }\end{array}$ & Resint & Duplicate & Mran \\
\hline \multicolumn{2}{|c|}{ 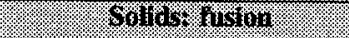 } & 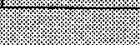 & 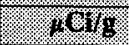 & .14016 & 1. \\
\hline S96T003143 & $146: 1$ & Upper $1 / 2$ & 5.79 & 6.32 & 6.055 \\
\hline S96T003144 & \multirow[t]{2}{*}{ 146: 2} & Upper $1 / 2$ & 5.89 & 6.34 & 6.115 \\
\hline S96T003145 & & Lower $1 / 2$ & 1.81 & 1.73 & 1.77 \\
\hline S96T003146 & \multirow[t]{2}{*}{ 146: 3} & Upper $1 / 2$ & 6.07 & 6.36 & 6.215 \\
\hline S96T003147 & & Lower $1 / 2$ & 1.86 & 2.01 & $1 . \overline{935}$ \\
\hline S96T003001 & $146: 3 A$ & Upper $1 / 2$ & 4.85 & 4.75 & 4.80 \\
\hline S96T003002 & \multirow[t]{2}{*}{$146: 4$} & Upper $1 / 2$ & 3.94 & 3.93 & 3.935 \\
\hline S96T003003 & & Lower $1 / 2$ & 8.38 & 8.34 & 8.36 \\
\hline S96T003004 & \multirow[t]{2}{*}{$146: 5$} & Upper $1 / 2$ & 4.98 & 4.76 & 4.87 \\
\hline S96T003005 & & Lower $1 / 2$ & 7.19 & 7.15 & 7.17 \\
\hline S96T003006 & \multirow[t]{2}{*}{ 146: 6} & Upper $1 / 2$ & 7.06 & 7.5 & 7.28 \\
\hline S96T003007 & & Lower $1 / 2$ & 7.6 & 7.65 & $7 . \overline{625}$ \\
\hline S96T003008 & \multirow[t]{2}{*}{ 146: 7} & Upper $1 / 2$ & 8.27 & 8.74 & 8.505 \\
\hline S96T003009 & & Lower $1 / 2$ & 11.1 & 11.2 & 11.15 \\
\hline S96T003148 & \multirow[t]{2}{*}{$146: 8$} & Upper $1 / 2$ & 10.5 & 11.6 & 11.05 \\
\hline S96T003149 & & Lower $1 / 2$ & 17.1 & 17.2 & 17.15 \\
\hline S96T004180 & \multirow[t]{2}{*}{$146: 9$} & Lower $1 / 2$ & 51.8 & 56.7 & 54.25 \\
\hline S96T003150 & & Upper $1 / 2$ & 45.5 & 46.3 & 45.90 \\
\hline S96T003450 & Core 141 & $\begin{array}{l}\text { Solid } \\
\text { composite }\end{array}$ & 7.35 & 6.43 & $6 . \overline{89}$ \\
\hline S96T003660 & Core 145 & $\begin{array}{l}\text { Solid } \\
\text { composite }\end{array}$ & 13.8 & $8.5 \overline{6}$ & 11.18 \\
\hline S96T004199 & Core 146 & $\begin{array}{l}\text { Solid } \\
\text { composite }\end{array}$ & 9.65 & 10.1 & 9.875 \\
\hline
\end{tabular}

Table B2-53. Tank 241-U-108 Grab Sample Analytical Results: Strontium-89/90.

\begin{tabular}{|c|c|c|c|c|c|}
\hline $\begin{array}{l}\text { Suringlo. } \\
\text { Tumber }\end{array}$ & Goringle & $\begin{array}{l}\text { Sorrigle } \\
\text { Iorgion }\end{array}$ & Reralus & Guplticate & Mean \\
\hline \multicolumn{2}{|c|}{ : Wrinds } & & 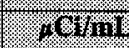 & I CWmir: & 1. $17 \mathrm{nI}$ \\
\hline S95T000978 & Riser 7 & Grab sample & 0.356 & 0.333 & $0.3445^{\text {QC:f }}$ \\
\hline
\end{tabular}




\section{B2.5.3 Total Beta}

Total beta activity was measured on the fusion digest of all core composite samples according to procedure LA-508-101, Rev. D-2 in support of the historical DQD. The results are shown in Table B2-54. Total beta activity ranged from 158.5 to $180.5 \mu \mathrm{Ci} / \mathrm{g}$.

Table B2-54. Tank 241-U-108 Core Sample Analytical Results: Total Beta.

\begin{tabular}{|c|c|c|c|c|c|}
\hline orample & Sorng & $\begin{array}{l}\text { Sarvple } \\
\text { Portion }\end{array}$ & Result & Guplizare & Mean \\
\hline \multicolumn{3}{|c|}{ Golfis rusien } & 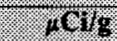 & IOH & Hovg \\
\hline S96T003450 & Core 141 & $\begin{array}{l}\text { Solid } \\
\text { composite }\end{array}$ & 191 & 170 & 180.5 \\
\hline S96T003660 & Core 145 & $\begin{array}{l}\text { Solid } \\
\text { composite }\end{array}$ & 194 & 123 & $158.5^{\mathrm{QCe}}$ \\
\hline S96T004199 & Core 146 & $\begin{array}{l}\text { Solid } \\
\text { composite }\end{array}$ & 165 & 157 & 161 \\
\hline
\end{tabular}

\section{B2.5.4 Total Uranium}

The concentration of uranium was determined by laser phosphorescence on fusion digests of the three core composite samples according to procedure LA-925-009, Rev. A-1. Results ranged from 140 (core 145) to $245 \mu \mathrm{g} / \mathrm{g}$ (core 146) and are shown in Table B2-55.

Table B2-55. Tank 241-U-108 Core Sample Analytical Results: Total Uranium.

\begin{tabular}{|c|c|c|c|c|c|}
\hline $\begin{array}{l}\text { Simople: } \\
\text { Number }\end{array}$ & Sample & $\begin{array}{l}\text { Sample } \\
\text { Poutum }\end{array}$ & Resull & Uublicate & Mear \\
\hline \multicolumn{2}{|c|}{ Soldos Gusion } & يis & (2. & . $186 \%$ & .198 \\
\hline S96T003450 & Core 141 & $\begin{array}{l}\text { Solid } \\
\text { composite }\end{array}$ & 192 & 162 & $177^{\mathrm{QC}: \mathrm{c}}$ \\
\hline S96T003660 & Core 145 & $\begin{array}{l}\text { Solid } \\
\text { composite }\end{array}$ & 134 & 146 & 140 \\
\hline S96T004199 & Core 146 & $\begin{array}{l}\text { Solid } \\
\text { composite }\end{array}$ & 230 & 260 & $245^{Q C: e}$ \\
\hline
\end{tabular}




\section{B2.5.5 Plutonium-239/240}

The activities of $\mathrm{Pu}-239$ and $\mathrm{Pu}-240$ were determined simultaneously by chemical separation followed by alpha counting. Only the grab sample was analyzed for Pu-239/240 using procedure LA-943-127, Rev. A-0. Results were below the detection limit of 6.8 E-05 and are shown in Table B2-56.

Table B2-56. Tank 241-U-108 Grab Sample Analytical Results: Plutonium-239/40.

\begin{tabular}{|c|c|c|c|c|c|}
\hline Wamilo & Womolo & Womplo & 19sinil. & Bunlare & $1 / 411$ \\
\hline \multicolumn{2}{|c|}{ Wrailis. } & & 16111\% & WOH & $40 \% 111$ \\
\hline S95T000978 & Riser 7 & $\begin{array}{l}\text { Grab } \\
\text { sample }\end{array}$ & $<6.840 \mathrm{E}-05$ & $<9.330 \mathrm{E}-05$ & $<8.085 \mathrm{E}-05$ \\
\hline
\end{tabular}

\section{B2.5.6 Americium-241}

The Am-241 activity of grab sample U-108-1 was determined by chemical separation followed by alpha counting per procedure LA-953-103, Rev. A-3. Core samples were analyzed for Am-241 by GEA on an opportunistic basis (Kristofzski 1996) as discussed in Section B2.6. Results are shown in Table B2-57.

Table B2-57. Tank 241-U-108 Grab Sample Analytical Results: Americium-241.

\begin{tabular}{|c|c|c|c|c|c|}
\hline W11mor & oogingen & 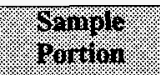 & istant & Hinlicile & Mran \\
\hline 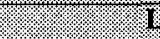 & MV & & H. Win & 16\%111. & IOY \\
\hline S95T000978 & Riser 7 & Grab sample & 0.00235 & 0.00244 & 0.002395 \\
\hline
\end{tabular}

\section{B2.6 GAMMA ENERGY ANALYSIS}

Although the activities of selected gamma-emitting radionuclides on solid core samples were determined by GEA, only the analyte Cs-137 was required by the core SAP. All analyses on the core samples were performed on fusion digests. Additional GEA data were collected on an opportunistic basis and are reported here. All GEA results are presented in Tables B2-58 through B2-63. Because data other than Cs-137 were not identified in any DQO document, there were no programmatic $\mathrm{QC}$ requirements with respect to these data, and the $\mathrm{QC}$ information associated with them were not evaluated for this report. 


\section{B2.6.1 Cesium-137}

The Cs-137 activity of the liquid grab sample was determined using procedure LA-548-121, Rev. D-1. At $416 \mu \mathrm{Ci} / \mathrm{mL}$, the grab sample analyzed had roughly twice the Cs- 137 activity as any core sample. The Cs-137 activities of the 1996 core samples were determined using procedure LA-548-121, Rev. E-0 and were comparable from segment to segment and core to core except for one sample (upper half of segment 9, core 141) that had an unusually low activity. With the exception of that sample, activities ranged from a low of $75 \mu \mathrm{Ci} / \mathrm{g}$ (lower half of segment 2 , core 146 ) to a high of $206 \mu \mathrm{Ci} / \mathrm{g}$ (upper half of segment 8 , core 141 ).

Table B2-58. Tank 241-U-108 Core Sample Analytical Results: Cesium-137 (GEA). (2 sheets)

\begin{tabular}{|c|c|c|c|c|c|}
\hline 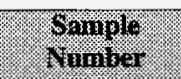 & $\begin{array}{l}\text { Saruplo } \\
\text { rogatirion }\end{array}$ & Somple & Rerail & Dippllcate & Merio \\
\hline \multicolumn{2}{|c|}{ 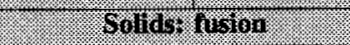 } & (3) & 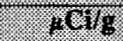 & $12 \mathrm{CI}$ & 1949 \\
\hline S96T002257 & 141: 1 & Lower $1 / 2$ & 180 & 205 & 192.5 \\
\hline S96T002258 & 141: 2 & Upper $1 / 2$ & 130.6 & 135 & 132.8 \\
\hline S96T002259 & 141: 3 & Upper $1 / 2$ & 129.7 & 128 & 128.85 \\
\hline S96T002260 & 141: $3 \mathrm{~A}$ & Upper $1 / 2$ & 119.7 & 113 & 116.35 \\
\hline S96T002261 & $141: 4$ & Upper $1 / 2$ & 166.5 & 182 & 174.25 \\
\hline S96T002262 & & Lower $1 / 2$ & 196.4 & 194 & 195.2 \\
\hline S96T002263 & $141: 4 \mathrm{~A}$ & Upper $1 / 2$ & 184.3 & 187 & $18 \overline{5.65}$ \\
\hline S96T002264 & & Lower $1 / 2$ & 167.5 & 191 & 179.25 \\
\hline S96T002608 & $141: 5$ & Upper $1 / 2$ & 168.9 & 171 & 169.95 \\
\hline S96T002609 & & Lower $1 / 2$ & 135.6 & 143 & 139.3 \\
\hline S96T002610 & $141: 6$ & Upper $1 / 2$ & 132.9 & 127 & 129.95 \\
\hline S96T002611 & & Lower $1 / 2$ & 130.4 & 127 & $\overline{128.7}$ \\
\hline S96T002612 & $141: 7$ & Upper $1 / 2$ & 204.6 & 182 & 193.3 \\
\hline S96T002613 & & Lower $1 / 2$ & 173.2 & 150 & 161.6 \\
\hline S96T002614 & 141: 8 & Upper $1 / 2$ & 200.4 & 212 & 206.2 \\
\hline S96T002615 & & Lower $1 / 2$ & 149.2 & 156 & 152.6 \\
\hline S96T002616 & 141: 9 & Upper $1 / 2$ & 43.96 & 44.1 & 44.03 \\
\hline S96T002909 & 145: 2 & Upper $1 / 2$ & 97.18 & 99.8 & 98.49 \\
\hline S96T002910 & $145: 3$ & Upper $1 / 2$ & 124.1 & 126 & 125.05 \\
\hline S96T002911 & & Lower $1 / 2$ & 126.6 & 122 & 124.3 \\
\hline S96T002912 & $145: 4$ & Upper $1 / 2$ & 123.4 & 118 & 120.7 \\
\hline S96T002913 & & Lower $1 / 2$ & 153.2 & 151 & 152.1 \\
\hline
\end{tabular}


Table B2-58. Tank 241-U-108 Core Sample Analytical Results: Cesium-137 (GEA). ( 2 sheets)

\begin{tabular}{|c|c|c|c|c|c|}
\hline sample & $\begin{array}{l}\text { Samples } \\
\text { I roartion }\end{array}$ & $\begin{array}{l}\text { Gomple } \\
\text { Sortion }\end{array}$ & Resill & Brylicans & Mena \\
\hline \multicolumn{2}{|c|}{ C. Solids: Gision : } & 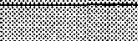 & . & 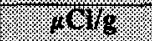 & 1.59 \\
\hline S96T002914 & $145: 5$ & Upper $1 / 2$ & 133.7 & 139 & 136.35 \\
\hline S96T002915 & & Lower $1 / 2$ & 199 & 196 & 197.5 \\
\hline S96T002916 & 145: 6 & Upper $1 / 2$ & 157.4 & 147 & 152.2 \\
\hline S96T002917 & & Lower $1 / 2$ & 141 & 144 & 142.5 \\
\hline S96T002918 & $145: 7$ & Upper $1 / 2$ & 152.1 & 149 & 150.55 \\
\hline S96T002919 & & Lower $1 / 2$ & 167.1 & 167 & 167.05 \\
\hline S96T002920 & 145: 8 & Upper $1 / 2$ & 98.75 & 97.2 & 97.97 \\
\hline S96T002921 & & Lower $1 / 2$ & 114.7 & 129 & 121.85 \\
\hline S96T002922 & 145: 9 & Upper $1 / 2$ & 130.8 & 128 & 129.4 \\
\hline S96T002923 & & Lower $1 / 2$ & 80.92 & 87.3 & 84.11 \\
\hline S96T003143 & 146: 1 & Upper $1 / 2$ & 152.1 & 163 & 157.55 \\
\hline S96T003144 & 146: 2 & Upper $1 / 2$ & 103.3 & 109 & 106.15 \\
\hline S96T003145 & & Lower $1 / 2$ & 73.32 & 76.9 & 75.11 \\
\hline S96T003146 & 146: 3 & Upper $1 / 2$ & 105.1 & 97.4 & 101.25 \\
\hline S96T003147 & & Lower $1 / 2$ & 89.54 & 89.8 & 89.67 \\
\hline S96T003001 & 146: $3 A$ & Upper $1 / 2$ & 98.49 & 93.2 & 95.84 \\
\hline S96T003002 & $146: 4$ & Upper $1 / 2$ & 100 & 100 & 100 \\
\hline S96T003003 & & Lower $1 / 2$ & 151.8 & 147 & 149.4 \\
\hline S96T003004 & $146: 5$ & Upper $1 / 2$ & 175.3 & 180 & 177.65 \\
\hline S96T003005 & & Lower $1 / 2$ & 137.2 & 139 & 138.1 \\
\hline S96T003006 & 146: 6 & Upper $1 / 2$ & 150.6 & 142 & 146.3 \\
\hline S96T003007 & & Lower $1 / 2$ & 163.2 & 157 & 160.1 \\
\hline S96T003008 & 146: 7 & Upper $1 / 2$ & 170.9 & 175 & 172.95 \\
\hline S96T003009 & & Lower $1 / 2$ & 188.8 & 197 & 192.9 \\
\hline S96T003148 & 146: 8 & Upper $\overline{1 / 2}$ & 183.2 & 188 & 185.6 \\
\hline S96T003149 & & Lower $1 / 2$ & 184.5 & 192 & 188.25 \\
\hline S96T004180 & 146: 9 & Lower $1 / 2$ & 96.75 & 94.5 & 95.63 \\
\hline S96T003150 & & Upper $1 / 2$ & 166.4 & 161 & 163.7 \\
\hline S96T003450 & Core 141 & $\begin{array}{l}\text { Solid } \\
\text { composite }\end{array}$ & 207.1 & 173 & 190.05 \\
\hline S96T003660 & Core 145 & $\begin{array}{l}\text { Solid } \\
\text { composite }\end{array}$ & 180 & 116 & $148^{\mathrm{QC}: \mathrm{e}}$ \\
\hline S96T004199 & Core 146 & \begin{tabular}{|l}
$\begin{array}{l}\text { Solid } \\
\text { composite }\end{array}$ \\
\end{tabular} & 149 & 149 & 149 \\
\hline
\end{tabular}


Table B2-59. Tank 241-U-108 Grab Sample Analytical Results: Cesium-137 (GEA).

\begin{tabular}{|c|c|c|c|c|c|}
\hline $\begin{array}{l}86 \text { mole } \\
\text { vimiser }\end{array}$ & $\begin{array}{l}\text { samils } \\
\text { yrachlion }\end{array}$ & $\begin{array}{l}\text { Sainplo } \\
\text { Pontion }\end{array}$ & Resull & Buplicate & Mean \\
\hline \multicolumn{3}{|c|}{ 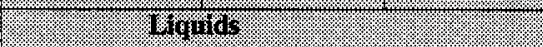 } & $121 \mathrm{~min}$ & WVirit. & Hormu \\
\hline S95T000978 & Riser 7 & Grab sample & 418 & 414 & 416 \\
\hline
\end{tabular}

Table B2-60. Tank 241-U-108 Core Sample Analytical Results: Americium-241 (GEA). ( 2 sheets)

\begin{tabular}{|c|c|c|c|c|c|}
\hline Sormifo & Somile & $\begin{array}{l}\text { Sranple } \\
\text { romerion. }\end{array}$ & Arevilit: & Bupllicate & Mean \\
\hline \multicolumn{2}{|c|}{ 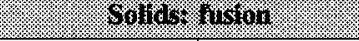 } & & 498 & I बirg & mas \\
\hline S96T002257 & 141: 1 & Lower $1 / 2$ & $<1.111$ & $<1.18$ & $<1.146$ \\
\hline S96T002258 & 141: 2 & Upper $1 / 2$ & $<0.9717$ & $<0.995$ & $<0.9834$ \\
\hline S96T002259 & $141: 3$ & Upper $1 / 2$ & $<0.6591$ & $<0.651$ & $<0.6550$ \\
\hline S96T002260 & $141: 3 A$ & Upper $1 / 2$ & $<0.09824$ & $<0.0944$ & $<0.0963$ \\
\hline S96T002261 & \multirow[t]{2}{*}{ 141: 4} & Upper $1 / 2$ & $<0.1118$ & $<0.115$ & $<0.1134$ \\
\hline S96T002262 & & Lower $1 / 2$ & $<0.1253$ & $<0.121$ & $<0.1232$ \\
\hline S96T002263 & \multirow[t]{2}{*}{ 141: 4A } & Upper $1 / 2$ & $<1.115$ & $<1.14$ & $<1.1275$ \\
\hline S96T002264 & & Lower $1 / 2$ & $<1.062$ & $<1.13$ & $<1.096$ \\
\hline S96T002608 & \multirow[t]{2}{*}{ 141: 5} & Upper $1 / 2$ & $<0.4211$ & $<0.427$ & $<0.4241$ \\
\hline S96T002609 & & Lower $1 / 2$ & $<0.4119$ & $<0.405$ & $<0.4084$ \\
\hline S96T002610 & \multirow[t]{2}{*}{ 141: 6} & Upper $1 / 2$ & $<0.3983$ & $<0.375$ & $<0.3866$ \\
\hline S96T002611 & & Lower $1 / 2$ & $<0.4007$ & $<0.4$ & $<0.4003$ \\
\hline S96T002612 & \multirow[t]{2}{*}{$141: 7$} & Upper $1 / 2$ & $<1.268$ & $<1.2$ & $<1.234$ \\
\hline S96T002613 & & Lower $1 / 2$ & $<1.242$ & $<1.13$ & $<1.186$ \\
\hline S96T002614 & \multirow[t]{2}{*}{ 141: 8} & Upper $1 / 2$ & $<1.302$ & $<1.26$ & $<1.281$ \\
\hline S96T002615 & & Lower $1 / 2$ & $<1.144$ & $<1.08$ & $<1.112$ \\
\hline S96T002616 & 141: 9 & Upper $1 / 2$ & $<0.6329$ & $<0.622$ & $<0.6275$ \\
\hline S96T002909 & $145: 2$ & Upper $1 / 2$ & $<0.7227$ & $<0.72$ & $<0.7214$ \\
\hline S96T002910 & \multirow[t]{2}{*}{$145: 3$} & Upper $1 / 2$ & $<0.8062$ & $<0.829$ & $<0.8176$ \\
\hline S96T002911 & & Lower $1 / 2$ & $<0.822$ & $<0.836$ & $<0.829$ \\
\hline S96T002912 & \multirow[t]{2}{*}{$145: 4$} & Upper $1 / 2$ & $<0.7187$ & $<0.699$ & $<0.7088$ \\
\hline S96T002913 & & Lower $1 / 2$ & $<0.8876$ & $<0.911$ & $<0.8993$ \\
\hline S96T002914 & \multirow[t]{2}{*}{$145: 5$} & Upper $1 / 2$ & $<0.8668$ & $<0.886$ & $<0.8764$ \\
\hline S96T002915 & & Lower $1 / 2$ & $<0.352$ & $<0.348$ & $<0.3500$ \\
\hline
\end{tabular}


Table B2-60. Tank 241-U-108 Core Sample Analytical Results: Americium-241 (GEA). ( 2 sheets)

\begin{tabular}{|c|c|c|c|c|c|}
\hline $\begin{array}{l}\text { Gimple. } \\
\text { Kumizen }\end{array}$ & S Samplét & $\begin{array}{l}\text { Sample } \\
\text { Portion }\end{array}$ & Meswiti. & Binilicite & Mean \\
\hline \multicolumn{2}{|c|}{ Sollus histor : } & & 12018 & $14 \% 16$ & 1901/8 \\
\hline S96T002916 & \multirow[t]{2}{*}{ 145: 6} & Upper $1 / 2$ & $<0.3038$ & $<0.295$ & $<0.2994$ \\
\hline S96T002917 & & Lower $1 / 2$ & $<0.2804$ & $<0.283$ & $<0.2817$ \\
\hline S96T002918 & \multirow[t]{2}{*}{$145: 7$} & Upper $1 / 2$ & $<0.7944$ & $<0.786$ & $<0.7902$ \\
\hline S96T002919 & & Lower $1 / 2$ & $<0.8498$ & $<0.822$ & $<0.8359$ \\
\hline S96T002920 & \multirow[t]{2}{*}{$145: 8$} & Upper $1 / 2$ & $<0.6337$ & $<0.608$ & $<0.6208$ \\
\hline S96T002921 & & Lower $1 / 2$ & $<0.2579$ & $<0.272$ & $<0.2649$ \\
\hline S96T002922 & \multirow[t]{2}{*}{$145: 9$} & Upper $1 / 2$ & $<0.3226$ & $<0.32$ & $<0.3213$ \\
\hline S96T002923 & & Lower $1 / 2$ & $<0.2151$ & $<0.222$ & $<0.2185$ \\
\hline S96T003143 & 146: 1 & Upper $1 / 2$ & $<0.3575$ & $<0.376$ & $<0.3667$ \\
\hline S96T003144 & \multirow[t]{2}{*}{ 146: 2} & Upper $1 / 2$ & $<0.2749$ & $<0.26$ & $<0.2674$ \\
\hline S96T003145 & & Lower $1 / 2$ & $<0.1957$ & $<0.226$ & $<0.2108$ \\
\hline S96T003146 & \multirow[t]{2}{*}{ 146: 3} & Upper $1 / 2$ & $<0.6593$ & $<0.65$ & $<0.6546$ \\
\hline S96T003147 & & Lower $1 / 2$ & $<0.5748$ & $<0.581$ & $<0.5779$ \\
\hline S96T003001 & 146: $3 A$ & Upper $1 / 2$ & $<0.6375$ & $<0.613$ & $<0.6253$ \\
\hline S96T003002 & \multirow[t]{2}{*}{$146: 4$} & Upper $1 / 2$ & $<0.2443$ & $<0.25$ & $<0.2471$ \\
\hline S96T003003 & & Lower $1 / 2$ & $<0.344$ & $<0.343$ & $<0.3435$ \\
\hline S96T003004 & \multirow[t]{2}{*}{$146: 5$} & Upper $1 / 2$ & $<0.3912$ & $<0.369$ & $<0.3801$ \\
\hline S96T003005 & & Lower $1 / 2$ & $<0.8673$ & $<0.852$ & $<0.8596$ \\
\hline S96T003006 & \multirow[t]{2}{*}{ 146: 6} & Upper $1 / 2$ & $<0.9512$ & $<0.845$ & $<0.8981$ \\
\hline S96T003007 & & Lower $1 / 2$ & $<0.9484$ & $<0.932$ & $<0.9402$ \\
\hline S96T003008 & \multirow[t]{2}{*}{ 146: 7} & Upper $1 / 2$ & $<0.9464$ & $<0.972$ & $<0.9592$ \\
\hline S96T003009 & & Lower $1 / 2$ & $<0.9729$ & $<1.09$ & $<1.0314$ \\
\hline S96T003148 & \multirow[t]{2}{*}{ 146: 8} & Upper $1 / 2$ & $<0.9722$ & $<0.999$ & $<0.9856$ \\
\hline S96T003149 & & Lower $1 / 2$ & $<0.9528$ & $<0.967$ & $<0.9599$ \\
\hline S96T004180 & \multirow[t]{2}{*}{$146: 9$} & Lower $1 / 2$ & $<0.584$ & $<0.505$ & $<0.5445$ \\
\hline S96T003150 & & Upper $1 / 2$ & $<0.979$ & $<0.941$ & $<0.9600$ \\
\hline S96T003450 & Core 141 & $\begin{array}{l}\text { Solid } \\
\text { composite }\end{array}$ & $<0.8692$ & $<0.808$ & $<0.8386$ \\
\hline S96T003660 & Core 145 & $\begin{array}{l}\text { Solid } \\
\text { composite }\end{array}$ & $<0.843$ & $<0.602$ & $<0.7225$ \\
\hline S96T004199 & Core 146 & $\begin{array}{l}\text { Solid } \\
\text { composite }\end{array}$ & $<0.9072$ & $<0.885$ & $<0.8961$ \\
\hline
\end{tabular}


Table B2-61. Tank 241-U-108 Core Sample Analytical Results: Cobalt-60 (GEA). (2 sheets)

\begin{tabular}{|c|c|c|c|c|c|}
\hline $\begin{array}{l}\text { Sorpole } \\
\text { Yominer. }\end{array}$ & S & $\begin{array}{l}\text { Sample } \\
\text { rotion }\end{array}$ & Resullil & Ouplicare & Wean \\
\hline \multicolumn{2}{|c|}{ S Solitsy fision. } & 12 & .2918 & .8218 & $18 \mathrm{CH}_{6}$ \\
\hline S96T002257 & 141: 1 & Lower $1 / 2$ & $<0.03376$ & $<0.0326$ & $<0.03318$ \\
\hline S96T002258 & $141: 2$ & Upper $1 / 2$ & $<0.02556$ & $<0.0315$ & $<0.02853$ \\
\hline S96T002259 & 141: 3 & Upper $1 / 2$ & $<0.01436$ & $<0.0139$ & $<0.01413$ \\
\hline S96T002260 & 141: $3 \mathrm{~A}$ & Upper $1 / 2$ & $<0.02614$ & $<0.0236$ & $<0.02487$ \\
\hline S96T002261 & \multirow[t]{2}{*}{ 141: 4} & Upper $1 / 2$ & $<0.02212$ & $<0.0219$ & $<0.02201$ \\
\hline S96T002262 & & Lower $1 / 2$ & $<0.02719$ & $<0.0253$ & $<0.02624$ \\
\hline S96T002263 & \multirow[t]{2}{*}{ 141: 4A } & Upper $1 / 2$ & $<0.02928$ & $<0.0273$ & $<0.02829$ \\
\hline S96T002264 & & Lower 1/2 & $<0.03441$ & $<0.0289$ & $<0.03165$ \\
\hline S96T002608 & \multirow[t]{2}{*}{$141: 5$} & Upper $1 / 2$ & $<0.01565$ & $<0.0187$ & $<0.01717$ \\
\hline S96T002609 & & Lower $1 / 2$ & $<0.02093$ & $<0.0178$ & $<0.01936$ \\
\hline S96T002610 & \multirow[t]{2}{*}{ 141: 6} & Upper $1 / 2$ & $<0.01802$ & $<0.0168$ & $<0.01741$ \\
\hline S96T002611 & & Lower $1 / 2$ & $<0.01752$ & $<0.0177$ & $<0.01761$ \\
\hline S96T002612 & \multirow[t]{2}{*}{ 141: 7} & Upper $1 / 2$ & $<0.02473$ & $<0.0308$ & $<0.02776$ \\
\hline S96T002613 & & Lower $1 / 2$ & $<0.04274$ & $<0.0314$ & $<0.03707$ \\
\hline S96T002614 & \multirow[t]{2}{*}{$141: 8$} & Upper $1 / 2$ & $<0.03513$ & $<0.0373$ & $<0.03621$ \\
\hline S96T002615 & & Lower $1 / 2$ & $<0.04125$ & $<0.0352$ & $<0.03822$ \\
\hline S96T002616 & $141: 9$ & Upper $1 / 2$ & $<0.03695$ & $<0.0277$ & $<0.03232$ \\
\hline S96T002909 & 145: 2 & Upper $1 / 2$ & $<0.02819$ & $<0.0203$ & $<0.02424$ \\
\hline S96T002910 & \multirow[t]{2}{*}{$145: 3$} & Upper $1 / 2$ & $<0.02214$ & $<0.0321$ & $<0.02712$ \\
\hline S96T002911 & & Lower $1 / 2$ & $<0.02971$ & $<0.0235$ & $<0.02660$ \\
\hline S96T002912 & \multirow[t]{2}{*}{$145: 4$} & Upper $1 / 2$ & $<0.01854$ & $<0.0175$ & $<0.01802$ \\
\hline S96T002913 & & Lower $1 / 2$ & $<0.02807$ & $<0.0208$ & $<0.02443$ \\
\hline S96T002914 & \multirow[t]{2}{*}{$145: 5$} & Upper $1 / 2$ & $<0.02181$ & $<0.0238$ & $<0.02280$ \\
\hline S96T002915 & & Lower $1 / 2$ & $<0.0106$ & $<0.0114$ & $<0.01100$ \\
\hline S96T002916 & \multirow[t]{2}{*}{$145: 6$} & Upper $1 / 2$ & $<0.009969$ & $<0.0101$ & $<0.01003$ \\
\hline S96T002917 & & Lower $1 / 2$ & $<0.008876$ & $<0.00933$ & $<0.009103$ \\
\hline S96T002918 & \multirow[t]{2}{*}{ 145: 7} & Upper $1 / 2$ & $<0.01912$ & $<0.0186$ & $<0.01886$ \\
\hline S96T002919 & & Lower $1 / 2$ & $<0.01675$ & $<0.0191$ & $<0.01793$ \\
\hline S96T002920 & \multirow[t]{2}{*}{$145: 8$} & Upper $1 / 2$ & $<0.01612$ & $<0.0151$ & $<0.01561$ \\
\hline S96T002921 & & Lower $1 / 2$ & $<0.01043$ & $<0.0106$ & $<0.01051$ \\
\hline S96T002922 & \multirow[t]{2}{*}{$145: 9$} & Upper $1 / 2$ & 0.01897 & 0.0229 & 0.020935 \\
\hline S96T002923 & & Lower $1 / 2$ & $<0.009559$ & $<0.00829$ & $<0.008924$ \\
\hline S96T003143 & 146: 1 & Upper $1 / 2$ & $<0.01338$ & $<0.0125$ & $<0.01294$ \\
\hline
\end{tabular}


Table B2-61. Tank 241-U-108 Core Sample Analytical Results: Cobalt-60 (GEA). (2 sheets)

\begin{tabular}{|c|c|c|c|c|c|}
\hline Sample & $\begin{array}{l}\text { Simple } \\
\text { Encarion }\end{array}$ & $\begin{array}{l}\text { Sample } \\
\text { Portion }\end{array}$ & resuir & Buplicarte & Mean \\
\hline \multicolumn{2}{|c|}{ (1) solids heron : } & 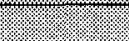 & 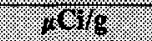 & Fing & 1018 \\
\hline S96T003144 & \multirow[t]{2}{*}{$146: 2$} & Upper $1 / 2$ & $<0.008736$ & $<0.00916$ & $<0.008948$ \\
\hline S96T003145 & & Lower $1 / 2$ & $<0.008127$ & $<0.0102$ & $<0.009163$ \\
\hline S96T003146 & \multirow[t]{2}{*}{ 146: 3} & Upper $1 / 2$ & $<0.01998$ & $<0.0204$ & $<0 . \overline{02019}$ \\
\hline S96T003147 & & Lower $1 / 2$ & $<0.01697$ & $<0.0144$ & $<0.01568$ \\
\hline S96T003001 & 146: $3 \mathrm{~A}$ & Upper $1 / 2$ & $<0.01673$ & $<0.0171$ & $<0.01691$ \\
\hline S96T003002 & \multirow[t]{2}{*}{ 146: 4} & Upper $1 / 2$ & $<0.009459$ & $<0.00977$ & $<0.009614$ \\
\hline S96T003003 & & Lower $1 / 2$ & $<0.01328$ & $<0.0135$ & $<0.01339$ \\
\hline S96T003004 & \multirow[t]{2}{*}{ 146: 5} & Upper $1 / 2$ & $<0.01252$ & $<0.0111$ & $<0.01181$ \\
\hline S96T003005 & & Lower $1 / 2$ & $<0.02485$ & $<0.0271$ & $<0.02597$ \\
\hline S96T003006 & \multirow[t]{2}{*}{ 146: 6} & Upper $1 / 2$ & $<0.01731$ & $<0.0154$ & $<0.01635$ \\
\hline S96T003007 & & Lower $1 / 2$ & $<0.02745$ & $<0.0198$ & $<0.02362$ \\
\hline S96T003008 & \multirow[t]{2}{*}{ 146: 7} & Upper $1 / 2$ & $<0.02409$ & $<0.0223$ & $<0.02319$ \\
\hline S96T003009 & & Lower $1 / 2$ & $<0.02213$ & $<0.0272$ & $<0.02466$ \\
\hline S96T003148 & \multirow[t]{2}{*}{$146: 8$} & Upper $1 / 2$ & $<0.01837$ & $<0.0172$ & $<0.01778$ \\
\hline S96T003149 & & Lower $1 / 2$ & $<0.02254$ & $<0.0159$ & $<0.01922$ \\
\hline S96T004180 & \multirow[t]{2}{*}{$146: 9$} & Lower $1 / 2$ & $<0.0446$ & $<0.038$ & $<0.04130$ \\
\hline S96T003150 & & Upper $1 / 2$ & $<0.02381$ & $<0.0225$ & $<0.02315$ \\
\hline S96T003450 & Core 141 & $\begin{array}{l}\text { Solid } \\
\text { composite }\end{array}$ & $<0 . \overline{01568}$ & $<0.0186$ & $<0.01714$ \\
\hline S96T003660 & Core 145 & $\begin{array}{l}\text { Solid } \\
\text { composite }\end{array}$ & $<0.02667$ & $<0.0102$ & $<0.01843$ \\
\hline S96T004199 & Core 146 & $\begin{array}{l}\text { Solid } \\
\text { composite }\end{array}$ & $<0.02152$ & $<0.0296$ & $<0.02556$ \\
\hline
\end{tabular}


Table B2-62. Tank 241-U-108 Core Sample Analytical Results: Europium-154 (GEA). (2 sheets)

\begin{tabular}{|c|c|c|c|c|c|}
\hline $\begin{array}{l}\text { Sample: } \\
\text { Nuniber. }\end{array}$ & Sample & $\begin{array}{l}\text { Samplo: } \\
\text { jortion. }\end{array}$ & Revil: & Buplicate & Menin \\
\hline \multicolumn{2}{|c|}{ 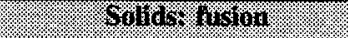 } & (3. & 1018 & 4016 & 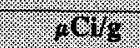 \\
\hline S96T002257 & 141: 1 & Lower $1 / 2$ & $<0.1333$ & $<0.118$ & $<0.12565$ \\
\hline S96T002258 & 141: 2 & Upper $1 / 2$ & $<0.1031$ & $<0.0962$ & $<0.09965$ \\
\hline S96T002259 & $141: 3$ & Upper $1 / 2$ & $<0.06633$ & $<0.0693$ & $<0.06781$ \\
\hline S96T002260 & 141: $3 \mathrm{~A}$ & Upper $1 / 2$ & $<0.08558$ & $<0.0791$ & $<0.08234$ \\
\hline S96T002261 & \multirow[t]{2}{*}{ 141: 4} & Upper $1 / 2$ & $<0.08931$ & $<0.0991$ & $<0.09420$ \\
\hline S96T002262 & & Lower $1 / 2$ & $<0.1042$ & $<0.098$ & $<0.10110$ \\
\hline S96T002263 & \multirow[t]{2}{*}{ 141: $4 \mathrm{~A}$} & Upper $1 / 2$ & $<0.1175$ & $<0.117$ & $<0.11725$ \\
\hline S96T002264 & & Lower $1 / 2$ & $<0.0853$ & $<0.108$ & $<0.09665$ \\
\hline S96T002608 & \multirow[t]{2}{*}{$141: 5$} & Upper $1 / 2$ & $<0.0605$ & $<0.0655$ & $<0.06300$ \\
\hline S96T002609 & & Lower $1 / 2$ & $<0.06897$ & $<0.0606$ & $<0.06478$ \\
\hline S96T002610 & \multirow[t]{2}{*}{ 141: 6} & Upper $1 / 2$ & $<0.05389$ & $<0 . \overline{0546}$ & $<0.05424$ \\
\hline S96T002611 & & Lower $1 / 2$ & $<0.05852$ & $<0.061$ & $<0.05976$ \\
\hline S96T002612 & \multirow[t]{2}{*}{$141: 7$} & Upper $1 / 2$ & $<0.09173$ & $<0.0975$ & $<0.09461$ \\
\hline S96T002613 & & Lower $1 / 2$ & $<0.123$ & $<0.137$ & $<0.1300$ \\
\hline S96T002614 & \multirow[t]{2}{*}{ 141: 8} & Upper $1 / 2$ & $<0.1247$ & $<0.115$ & $<0.1198$ \\
\hline S96T002615 & & Lower $1 / 2$ & $<0.1788$ & $<0.124$ & $<0.1514$ \\
\hline S96T002616 & $141: 9$ & Upper $1 / 2$ & $<0.09579$ & $<0.0983$ & $<0.09705$ \\
\hline \$96Т002909 & $145: 2$ & Upper $1 / 2$ & $<0.07418$ & $<0.0876$ & $<0.08089$ \\
\hline S96T002910 & \multirow[t]{2}{*}{$145: 3$} & Upper $1 / 2$ & $<0.1008$ & $<0.0853$ & $<0.09305$ \\
\hline S96T002911 & & Lower $1 / 2$ & $<0.09677$ & $<0.106$ & $<0.10138$ \\
\hline S96T002912 & \multirow[t]{2}{*}{$145: 4$} & Upper $1 / 2$ & $<0.07392$ & $<0.064$ & $<0.06896$ \\
\hline S96T002913 & & Lower $1 / 2$ & $<0.08475$ & $<0.102$ & $<0.09337$ \\
\hline S96T002914 & \multirow[t]{2}{*}{$145: 5$} & Upper $1 / 2$ & $<0.08122$ & $<0.0958$ & $<0.08851$ \\
\hline S96T002915 & & Lower $1 / 2$ & $<0.04459$ & $<0.0431$ & $<0.04384$ \\
\hline S96T002916 & \multirow[t]{2}{*}{$145: 6$} & Upper $1 / 2$ & $<0.03425$ & $<0.0345$ & $<0.03437$ \\
\hline S96T002917 & & Lower $1 / 2$ & $<0.03012$ & $<0.0323$ & $<0.03121$ \\
\hline S96T002918 & \multirow[t]{2}{*}{$145: 7$} & Upper $1 / 2$ & $<0.06448$ & $<0.063$ & $<0.06374$ \\
\hline S96T002919 & & Lower $1 / 2$ & $<0.06277$ & $<0.0758$ & $<0.06928$ \\
\hline S96T002920 & \multirow[t]{2}{*}{$145: 8$} & Upper $1 / 2$ & $<0.04999$ & $<0.0551$ & $<0.05254$ \\
\hline S96T002921 & & Lower $1 / 2$ & $<0.03427$ & $<0.0374$ & $<0.03583$ \\
\hline S96T002922 & \multirow[t]{2}{*}{ 145: 9} & Upper $1 / 2$ & 0.1998 & 0.213 & 0.2064 \\
\hline S96T002923 & & Lower $1 / 2$ & 0.05363 & $<0.0328$ & $<0.04321$ \\
\hline
\end{tabular}


Table B2-62. Tank 241-U-108 Core Sample Analytical Results: Europium-154 (GEA). (2 sheets)

\begin{tabular}{|c|c|c|c|c|c|}
\hline $\begin{array}{l}\text { Sample. } \\
\text { Hiniler. }\end{array}$ & $\begin{array}{l}\text { Gample } \\
\text { socintion }\end{array}$ & $\begin{array}{l}\text { Sample } \\
\text { Coutrian }\end{array}$ & Resiat: & Buplicate & Mear \\
\hline Giolas & W8401 & & 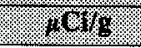 & 10919 & . \\
\hline S96T003143 & $146: 1$ & Upper $1 / 2$ & $<0.04867$ & $<0.0571$ & $<0.05288$ \\
\hline S96T003144 & \multirow[t]{2}{*}{ 146: 2} & Upper $1 / 2$ & 0.06233 & $<0.0382$ & $<0 . \overline{05026}$ \\
\hline S96T003145 & & Lower $1 / 2$ & $<0.02782$ & $<0.0307$ & $<0.02926$ \\
\hline S96T003146 & \multirow[t]{2}{*}{$146: 3$} & Upper $1 / 2$ & $<0.06112$ & $<0.0797$ & $<0.07041$ \\
\hline S96T003147 & & Lower $1 / 2$ & $<0.04996$ & $<0.0523$ & $<0.05113$ \\
\hline S96T003001 & 146: $3 A$ & Upper $1 / 2$ & $<0.07431$ & $<0.0664$ & $<0.07035$ \\
\hline S96T003002 & \multirow[t]{2}{*}{ 146: 4} & Upper $1 / 2$ & $<0.03901$ & $<0.0408$ & $<0.03990$ \\
\hline S96T003003 & & Lower $1 / 2$ & $<0.04918$ & $<0.0512$ & $<0.05019$ \\
\hline S96T003004 & \multirow[t]{2}{*}{$146: 5$} & Upper $1 / 2$ & $<0.04898$ & $<0.0465$ & $<0.04774$ \\
\hline S96T003005 & & Lower $1 / 2$ & $<0.08836$ & $<0.0866$ & $<0.08748$ \\
\hline S96T003006 & \multirow[t]{2}{*}{$146: 6$} & Upper $1 / 2$ & $<0.09557$ & $<0.0699$ & $<0.08273$ \\
\hline S96T003007 & & Lower $1 / 2$ & $<0.06238$ & $<0.0831$ & $<0.07274$ \\
\hline S96T003008 & \multirow[t]{2}{*}{$146: 7$} & Upper $1 / 2$ & $<0.08322$ & $<0.0622$ & $<0.07271$ \\
\hline S96T003009 & & Lower $1 / 2$ & $<0.0735$ & $<0.0997$ & $<0.0866$ \\
\hline S96T003148 & \multirow[t]{2}{*}{ 146: 8} & Upper $1 / 2$ & $<0.08311$ & $<0.085$ & $<0.08405$ \\
\hline S96T003149 & & Lower $1 / 2$ & $<0.07069$ & $<0.0868$ & $<0.07874$ \\
\hline S96T004180 & \multirow[t]{2}{*}{$146: 9$} & Lower $1 / 2$ & 0.3369 & $<0.145$ & $<0.24095$ \\
\hline S96T003150 & & Upper $1 / 2$ & $<0.1232$ & $<0.123$ & $<0.1231$ \\
\hline S96T003450 & Core 141 & $\begin{array}{l}\text { Solid } \\
\text { composite }\end{array}$ & $<0.07773$ & $<0.0678$ & $<0.07276$ \\
\hline S96T003660 & Core 145 & $\begin{array}{l}\text { Solid } \\
\text { composite }\end{array}$ & $<0.07935$ & $<0.0513$ & $<0.06532$ \\
\hline S96T004199 & Core 146 & $\begin{array}{l}\text { Solid } \\
\text { composite }\end{array}$ & $<0.09139$ & $<0.0659$ & $<0.07864$ \\
\hline
\end{tabular}


Table B2-63. Tank 241-U-108 Core Sample Analytical Results: Europium-155 (GEA). (2 sheets)

\begin{tabular}{|c|c|c|c|c|c|}
\hline $\begin{array}{l}\text { Sample: } \\
\text { Nominer }\end{array}$ & Sompile & Sariple & Resinil & Duplicate & Mean \\
\hline STIIdos & miston & (2.8. & 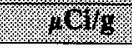 & (3) & 2018 \\
\hline S96T002257 & 141: 1 & Lower $1 / 2$ & $<0.425$ & $<0.453$ & $<0.4390$ \\
\hline S96T002258 & $141: 2$ & Upper $1 / 2$ & $<0.3674$ & $<0.387$ & $<0.3772$ \\
\hline S96T002259 & $141: 3$ & Upper $1 / 2$ & $<0.2494$ & $<0.246$ & $<0.2477$ \\
\hline S96T002260 & 141: $3 A$ & Upper $1 / 2$ & $<0.1804$ & $<0.172$ & $<0.1762$ \\
\hline S96T002261 & \multirow[t]{2}{*}{ 141: 4} & Upper $1 / 2$ & $<0.2043$ & $<0.213$ & $<0.2086$ \\
\hline S96T002262 & & Lower $1 / 2$ & $<0.2279$ & $<0.222$ & $<0.2249$ \\
\hline S96T002263 & \multirow[t]{2}{*}{ 141: 4A } & Upper $1 / 2$ & $<0.4257$ & $<0.435$ & $<0 . \overline{4303}$ \\
\hline S96T002264 & & Lower $1 / 2$ & $<0.4094$ & $<0.434$ & $<0.4217$ \\
\hline S96T002608 & \multirow[t]{2}{*}{$141: 5$} & Upper $1 / 2$ & $<0.1961$ & $<0.2$ & $<0.1980$ \\
\hline S96T002609 & & Lower $1 / 2$ & $<0.191$ & $<0.19$ & $<0.1905$ \\
\hline S96T002610 & \multirow[t]{2}{*}{$141: 6$} & Upper $1 / 2$ & $<0.18 \overline{62}$ & $<0.173$ & $<0.1796$ \\
\hline S96T002611 & & Lower $1 / 2$ & $<0.1848$ & $<0.184$ & $<0.1844$ \\
\hline S96T002612 & \multirow[t]{2}{*}{$141: 7$} & Upper $1 / 2$ & $<0.5999$ & $<0.575$ & $<0.5874$ \\
\hline S96T002613 & & Lower $1 / 2$ & $<0.5979$ & $<0.535$ & $<0.5664$ \\
\hline S96T002614 & \multirow[t]{2}{*}{ 141: 8} & Upper $1 / 2$ & $<0.6146$ & $<0.601$ & $<0.6078$ \\
\hline S96T002615 & & Lower $1 / 2$ & $<0.5449$ & $<0.523$ & $<0.5339$ \\
\hline S96T002616 & 141: 9 & Upper $1 / 2$ & $<0.3024$ & $<0.305$ & $<0.3037$ \\
\hline S96T002909 & $145: 2$ & Upper $1 / 2$ & $<0.3462$ & $<0.352$ & $<0.3491$ \\
\hline S96T002910 & \multirow[t]{2}{*}{$145: 3$} & Upper $1 / 2$ & $<0.3989$ & $<0.397$ & $<0.3979$ \\
\hline S96T002911 & & Lower $1 / 2$ & $<0.3958$ & $<0.391$ & $<0.3934$ \\
\hline S96T002912 & \multirow[t]{2}{*}{$145: 4$} & Upper $1 / 2$ & $<0.2811$ & $<0.27$ & $<0.2755$ \\
\hline S96T002913 & & Lower $1 / 2$ & $<0.3468$ & $<0.345$ & $<0.3459$ \\
\hline S96T002914 & \multirow[t]{2}{*}{$145: 5$} & Upper $1 / 2$ & $<0.3291$ & $<0.335$ & $<0.3320$ \\
\hline S96T002915 & & Lower $1 / 2$ & $<0.1635$ & $<0.163$ & $<0.1632$ \\
\hline S96T002916 & \multirow[t]{2}{*}{$145: 6$} & Upper $1 / 2$ & $<0.1414$ & $<0.136$ & $<0.1387$ \\
\hline S96T002917 & & Lower $1 / 2$ & $<0.1298$ & $<0.131$ & $<0.1304$ \\
\hline S96T002918 & \multirow[t]{2}{*}{$145: 7$} & Upper $1 / 2$ & $<0.3812$ & $<0.374$ & $<0.3776$ \\
\hline S96T002919 & & Lower $1 / 2$ & $<0.4044$ & $<0.397$ & $<0.4007$ \\
\hline S96T002920 & \multirow[t]{2}{*}{$145: 8$} & Upper $1 / 2$ & $<0.2989$ & $<0.294$ & $<0.2964$ \\
\hline S96T002921 & & Lower $1 / 2$ & $<0.12$ & $<0.125$ & $<0.1225$ \\
\hline S96T002922 & \multirow[t]{2}{*}{ 145: 9} & Upper $1 / 2$ & 0.2204 & $<0.15$ & $<0.1852$ \\
\hline S96T002923 & & Lower $1 / 2$ & $<0.1006$ & $<0.103$ & $<0.1018$ \\
\hline
\end{tabular}


Table B2-63. Tank 241-U-108 Core Sample Analytical Results: Europium-155 (GEA). (2 sheets)

\begin{tabular}{|c|c|c|c|c|c|}
\hline $\begin{array}{l}\text { Sample } \\
\text { Transer. }\end{array}$ & 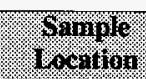 & $\begin{array}{l}\text { Sampio } \\
\text { Portion }\end{array}$ & Resint & Gupilicate & Mean. \\
\hline \multicolumn{2}{|c|}{ 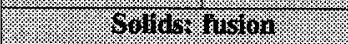 } & & $.30 \mathrm{gs}$ & 14018 & reflo \\
\hline S96T003143 & 146: 1 & Upper $1 / 2$ & $<0.1656$ & $<0.174$ & $<0.1698$ \\
\hline S96T003144 & \multirow[t]{2}{*}{ 146: 2} & Upper $1 / 2$ & $<0.1175$ & $<0.118$ & $<0.1177$ \\
\hline S96T003145 & & Lower $1 / 2$ & $<0.09056$ & $<0.102$ & $<0.09628$ \\
\hline S96T003146 & \multirow[t]{2}{*}{$146: 3$} & Upper $1 / 2$ & $<0.2538$ & $<0.246$ & $<0.2499$ \\
\hline S96T003147 & & Lower $1 / 2$ & $<0.2176$ & $<0.222$ & $<0.2198$ \\
\hline S96T003001 & 146: $3 A$ & Upper $1 / 2$ & $<0.2381$ & $<0.236$ & $<0.2370$ \\
\hline S96T003002 & \multirow[t]{2}{*}{$146: 4$} & Upper $1 / 2$ & $<0.111$ & $<0.114$ & $<0.1125$ \\
\hline S96T003003 & & Lower $1 / 2$ & $<0.1557$ & $<0.161$ & $<0.1583$ \\
\hline S96T003004 & \multirow[t]{2}{*}{ 146: 5} & Upper $1 / 2$ & $<0.1778$ & $<0.168$ & $<0.1729$ \\
\hline S96T003005 & & Lower $1 / 2$ & $<0.4228$ & $<0.408$ & $<0.4154$ \\
\hline S96T003006 & \multirow[t]{2}{*}{ 146: 6} & Upper $1 / 2$ & $<0.4551$ & $<0.41$ & $<0.4325$ \\
\hline S96T003007 & & Lower $1 / 2$ & $<0.4433$ & $<0.443$ & $<0.4431$ \\
\hline S96T003008 & \multirow[t]{2}{*}{ 146: 7} & Upper $1 / 2$ & $<0.4566$ & $<0.465$ & $<0.4608$ \\
\hline S96T003009 & & Lower $1 / 2$ & $<0.4654$ & $<0.52$ & $<0.4927$ \\
\hline S96T003148 & \multirow[t]{2}{*}{$146: 8$} & Upper $1 / 2$ & $<0.4664$ & $<0.476$ & $<0.4712$ \\
\hline S96T003149 & & Lower $1 / 2$ & $<0.3615$ & $<0.364$ & $<0.3627$ \\
\hline S96T004180 & \multirow[t]{2}{*}{ 146: 9} & Lower $1 / 2$ & $<0.2691$ & $<0.236$ & $<0.2525$ \\
\hline S96T003150 & & Üper $1 / 2$ & $<0.3816$ & $<0.367$ & $<0 . \overline{3743}$ \\
\hline S96T003450 & Core 141 & $\begin{array}{l}\text { Solid } \\
\text { composite }\end{array}$ & $<0.3341$ & $<0.309$ & $<0.3215$ \\
\hline S96T003660 & Core 145 & $\begin{array}{l}\text { Solid } \\
\text { composite }\end{array}$ & $<0.4058$ & $<0.29$ & $<0.3479$ \\
\hline S96T004199 & Core 146 & $\begin{array}{l}\text { Solid } \\
\text { composite }\end{array}$ & $<0.4305$ & $<0.429$ & $<0.4297$ \\
\hline
\end{tabular}

\section{B2.7 VAPOR PHASE MEASUREMENTS}

\section{B2.7.1 Safety Screening}

Before and during the April/May core sampling of tank 241-U-108, vapor phase measurements were made in support of the safety screening DQO. The vapor phase measurements reported here were taken in the tank headspace below a riser. Results were 
obtained in the field (i.e., no gas sample was sent to the laboratory for analysis). Multiple measurements were taken for each riser used for core sampling. The results in Table B2-64 are those with the highest LFL value for found for each riser. All data were reported in WHC (1996).

Table B2-64. Results of Vapor Phase Measurements of Tank 241-U-108.

\begin{tabular}{|c|c|c|c|}
\hline \multirow[t]{2}{*}{ Meavinenicin } & \multicolumn{3}{|c|}{ Terilis: } \\
\hline & 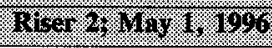 & 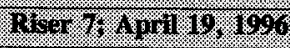 & Ruser 9.4 pril 26.1996 \\
\hline LFL & $4 \%$ of LFL & $3 \%$ of LFL & $6 \%$ of LFL \\
\hline TOC & $15 \mathrm{ppm}$ & $28 \mathrm{ppm}$ & $14 \mathrm{ppm}$ \\
\hline Oxygen & $20.8 \%$ & $20.8 \%$ & $21.0 \%$ \\
\hline Ammonia & $500 \mathrm{ppm}$ & $500 \mathrm{ppm}$ & $450 \mathrm{ppm}$ \\
\hline
\end{tabular}

\section{B2.7.2 1995 Tank Vapor Samples}

Data from the 1995 tank 241-U-108 vapor samples are shown in Table B2-65. Vapor samples were obtained in 1995 as required in Data Quality Objectives for Generic In-Tank Health and Safety Issue Resolution (Osborne et al. 1995). Since the 1995 vapor samples were taken, tank vapors are no longer being evaluated as a health concern (Hewitt 1996).

B2.7.2.1 Inorganic Analytes. The vapor concentrations of selected inorganic analytes, $\mathrm{NH}_{3}, \mathrm{NO}_{2}$, $\mathrm{NO}$, and vapor mass concentration (primarily $\mathrm{H}_{2} \mathrm{O}$ ), were determined. The average and one standard deviation of concentration results from inorganic sorbent sample trains used to sample headspace vapors were $692 \pm 7 \mathrm{ppmv}\left(\mathrm{NH}_{3}\right), \leq 0.02 \mathrm{ppmv}\left(\mathrm{NO}_{2}\right)$, $\leq$ $0.06 \mathrm{ppmv}(\mathrm{NO})$, and $15.5 \pm 0.1 \mathrm{mg} / \mathrm{L}$ (primarily $\mathrm{H}_{2} \mathrm{O}$ ). The vapor concentration results were based on six samples for each compound (eight samples for mass concentration). The $\mathrm{NO}_{2}$ and $\mathrm{NO}$ samples included four samples trailing (downstream of) $\mathrm{NH}_{3}$ sorbent traps and two samples unprotected by $\mathrm{NH}_{3}$ sorbent traps. All samples were successfully analyzed and used in the averages. Representative field blanks were also analyzed and used to correct data.

Two of the four average concentration results, $\mathrm{NH}_{3}$ and $\mathrm{H}_{2} \mathrm{O}$, exceeded the minimum of the expected ranges. The precision of results, based on one standard deviation of all samples, was $\leq 1$ percent (within the target level of \pm 25 percent) for analytes exceeding expected ranges. The estimated accuracies of vapor concentrations, assuming negligible sample volume uncertainty, were 90 to 110 percent (within the target range of 70 to 130 percent) for analytes exceeding the expected ranges. These uncertainties were confirmed by evaluation of spikes and continuing calibration standards $\left(\mathrm{NH}_{3}\right)$ and evaluation of the variability of field blanks $\left(\mathrm{H}_{2} \mathrm{O}\right)$. No procedural deviations were noted. Data and additional information on samples, analyses, and results are described in Thomas et al. (1996). 
B2.7.2.2 Permanent Gases. The complete results of the permanent-gas analysis of tank 241-U-108 can be found in Thomas et at. (1996). In summary, hydrogen (522 ppmv) and nitrous oxide (612 ppmv) were observed above the method detection limit (MDL) in the tank headspace samples, and carbon dioxide in the headspace samples was at a lower concentration than observed in the ambient air.

B2.7.2.3 Total Non-Methane Hydrocarbons. The complete results of the total non-methane hydrocarbons analysis of tank 241-U-108 can be found in Thomas et al. (1996). In summary, the average concentration in the three tank headspace samples was $11.99 \mathrm{mg} / \mathrm{m}^{3}$. This compares to $6.08 \mathrm{mg} / \mathrm{m}^{3}$ for the sum of all compounds identified in the target and tentatively identified compound (TID) analysis of the SUMMA ${ }^{\mathrm{TM}}$ canisters.

B2.7.2.4 Volatile Organic Analytes. The complete results of the volatile organic analysis of tank 241-U-108 can be also be found in Thomas et al. (1996). In summary, 14 target analytes above the 5-ppbv reporting cutoff and 12 tentatively identified compounds (TIDs) above the 10-ppbv reporting cutoff were detected in the tank headspace samples. Thirteen target analytes and all TIDs were identified in two or more tank headspace samples. The total concentration of the target analytes was found to be $3.47 \mathrm{mg} / \mathrm{m}^{3}$. The total TID concentration was found to be $2.60 \mathrm{mg} / \mathrm{m}^{3}$. The total concentration of all the compounds identified was $6.08 \mathrm{mg} / \mathrm{m}^{3}$. SUMMA ${ }^{\mathrm{Th}}$ canister PNL 48 was analyzed in replicate for target analytes and TIDs to determine analytical precision. Eight of 13 target analytes and 7 of 12 TIDs had RPDs of less than 10 percent. The compound 2-butanone was the only target analyte observed in the upwind ambient air sample. Acetone was observed in the ambient air through the VSS sample. No TIDs were observed in the two ambient air samples.

B2.7.2.5 Semi-Volatile Organic Analytes. The complete results of the sorbent trap analysis of tank 241-U-108 can be found in Thomas et al. (1996). In summary, 19 target analytes above the 5-ppbv reporting cutoff and 13 TIDs above the 10-ppbv reporting cutoff were detected in the tank headspace samples. Eighteen of 19 target analytes and 9 of 13 TIDs were observed in two or more sorbent traps. Two of 13 TIDs were identified as unknowns. The total concentration of the target analytes was found to be $4.49 \mathrm{mg} / \mathrm{m}^{3}$. The total concentration of the TIDs was found to be $4.25 \mathrm{mg} / \mathrm{m}^{3}$. The total concentration of all the compounds identified was $8.74 \mathrm{mg} / \mathrm{m}^{3}$. Triple sorbent trap sample PNL 635 was analyzed in replicate for target analytes and TIDs to determine analytical precision. Fifteen of 18 target analytes and 1 of 9 TIDs had RPDs of less than 10 percent. 
Table B2-65. Vapor Analytical Summary for Tank 241-U-108.

\begin{tabular}{|c|c|c|c|}
\hline Safey grom & Ifrget Anquition & 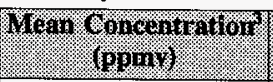 & 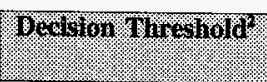 \\
\hline \multirow[t]{3}{*}{ Flammability } & Hydrogen & $522-2$ & $20 \%$ of LFL \\
\hline & Methane & $<25$ & $20 \%$ of LFL \\
\hline & Carbon monoxide & $<25$ & $20 \%$ of LFL \\
\hline \multirow{8}{*}{$\begin{array}{l}\text { Carcinogen } \\
\text { Teratogen } \\
\text { Mutagen }\end{array}$} & Acetonitrile & 0.029 & 250 \\
\hline & Benzene & 0.019 & 250 \\
\hline & 1,3-Butadiene & Not measured ${ }^{4}$ & 1000 \\
\hline & Hexane & 0.012 & 550 \\
\hline & Nitric oxide & $<0.06$ & 50 \\
\hline & Nitrogen dioxide & $<0.02$ & 10 \\
\hline & $\begin{array}{l}\mathrm{N} \text {-Nitrosodimethyl } \\
\text { amine }\end{array}$ & $0.051^{5}$ & $\begin{array}{l}\text { Any detectable } \\
\text { amount }^{6}\end{array}$ \\
\hline & Nitrous oxide & 612 & Not provided in SAP \\
\hline \multirow[t]{4}{*}{ Toxin } & Dodecane & Not detected & --- \\
\hline & Tridecane & Not detected & --- \\
\hline & Propane nitrile & Not detected & $\cdots$ \\
\hline & Carbon monoxide & $<25$ & $20 \%$ of $\mathrm{LFL}$ \\
\hline \multirow[t]{6}{*}{ Irritant } & Ammonia & 692 & 150 \\
\hline & Tributyl phosphate & Not measured ${ }^{4}$ & 15 \\
\hline & Carbon dioxide & $<25$ & Not provided in SAP \\
\hline & Butanol & 0.296 & 700 \\
\hline & Butanol & Not measured ${ }^{4}$ & $44,610 \mathrm{mg} / \mathrm{m}^{3} 7$ \\
\hline & Acetone & 0.25 & 1,250 \\
\hline
\end{tabular}

Notes:

${ }^{1}$ Mahlum et al. (1994)

${ }^{2}$ Homi (1995)

${ }^{3}$ Average of triple sorbent tube and SUMMA ${ }^{\mathrm{TM}}$ results

${ }^{4}$ Samples sent to PNNL for organic analysis were not analyzed for this compound. No sample was sent to Oak Ridge National Laboratory for organic analysis as required in the SAP.

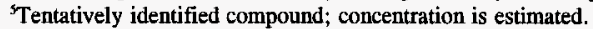

${ }^{6}$ The "immediately dangerous to life and health" concentration is not available for this compound. The National Institute for Occupational Safety and Health (NIOSH) recommends specific safeguards for any detectable amount of this carcinogen (NIOSH 1994).

7The "immediately dangerous to life and health" concentration is not available for this compound. The $\mathrm{LD}_{50}$ mouse inhalation value (Izmerov 1982) value was used. 


\section{B2.7.3 Flammable Gas Monitoring}

From June 1995 to May 1996, 14 headspace samples were removed from tank 241-U-108 using the Standard Hydrogen Monitoring System. The samples were analyzed for hydrogen, methane, and nitrous oxide. The samples were taken to support resolution of the flammable gas safety issue and to meet the requirements of Tri-Party Agreement (Ecology et al. 1996) milestone $\mathrm{M}-40-10$.

A description of the sampling methods and requirements is available in Wilkins et al. (1997). The analytical data are presented in Table B2-66. The results are consistent with the August 1995 and April/May 1996 vapor samples described in sections B2.7.1 and B2.7.2. The hydrogen concentrations reported in Wilkins et al. (1997) are well below 25 percent of the LFL, and the methane and nitrous oxide results compare well with the results from the August 1995 headspace samples.

Table B2-66. Tank 241-U-108 Flammable Gas Monitoring Vapor Sample Results.

\begin{tabular}{|c|c|c|c|}
\hline Sumple Dite & H. (ppm) & (6) (10m) & N. \\
\hline June 27,1995 & 430 & 11 & 330 \\
\hline June 30,1995 & 410 & 8 & 310 \\
\hline July 7, 1995 & 500 & 10 & 360 \\
\hline July 11,1995 & 470 & 10 & 530 \\
\hline July 14,1995 & 450 & 8 & $\overline{510}$ \\
\hline July 17,1995 & 430 & 18 & 450 \\
\hline September 11,1995 & 490 & 7 & 530 \\
\hline September 11, 1995 & 480 & 8 & 550 \\
\hline September 18,1995 & $\mathrm{n} / \mathrm{r}$ & $2^{1}$ & $\mathrm{n} / \mathrm{r}$ \\
\hline September 18, 1995 & 8 & 1 & $\mathrm{n} / \mathrm{r}$ \\
\hline February 7, 1996 & 296 & $\mathrm{n} / \mathrm{r}$ & 370 \\
\hline February 7, 1996 & 145 & $\mathrm{n} / \mathrm{r}$ & 120 \\
\hline May 9, 1996 & 520 & 10 & 600 \\
\hline May 9, 1996 & 530 & 20 & 600 \\
\hline
\end{tabular}

Notes:

$\mathrm{n} / \mathrm{r} \quad=\quad$ not reported

${ }^{1}$ The samples taken on September 18, 1995 are unusually low. A problem with the sample cylinder is suspected.

\section{B2.8 HISTORICAL SAMPLE RESULTS}

The results of the 1971,1973 or 1974, and 1975 samples are shown in Tables B2-67, B2-68, and B2-69. In the early to mid 1970 s, the contents of tank $241-\mathrm{U}-108$ were scheduled to be 
used as feed to the 242-S Evaporator. The 1971 sample was probably sludge since values for specific gravity and viscosity were not provided as with most other sample data given in Puryear (1971). None of the samples probably represent the present tank contents. These data have not been validated and should be used with caution.

Table B2-67. 1971 Sample., ${ }^{1,2}$

\begin{tabular}{|c|c|c|}
\hline & 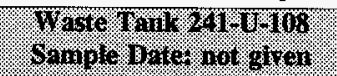 & \\
\hline 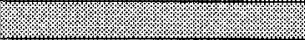 & 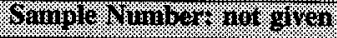 & \\
\hline WOMLOWIIII & 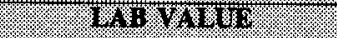 & WE WIVY \\
\hline 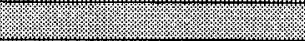 & 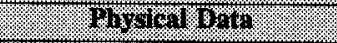 & \\
\hline 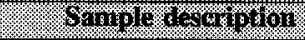 & \multicolumn{2}{|c|}{ rul orow wed } \\
\hline 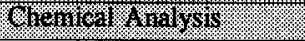 & 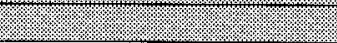 & \\
\hline $\mathrm{Al}$ & 0.25 & $M^{3}$ \\
\hline $\mathrm{Na}$ & 4.59 & $M^{3}$ \\
\hline $\mathrm{NO}_{2}$ & 0.231 & $\overline{M^{3}}$ \\
\hline $\mathrm{CO}_{3}$ & 0.086 & $M^{3}$ \\
\hline
\end{tabular}

Notes:

${ }^{1}$ Pre-1989 analytical data have not been validated and should be used with caution.

${ }^{2}$ Puryear (1971)

${ }^{3}$ Units were not provided in reference and are assumed to be molar.

Table B2-68. 1973 or 1974 Supernatant Sample. ${ }^{1,2}$ (2 sheets)

\begin{tabular}{|c|c|c|}
\hline & 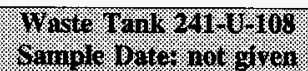 & \\
\hline & Saniple Timber T ThI & \\
\hline : .OMHONHKI & 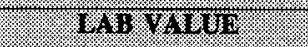 & 1.4. 3. U1\% \\
\hline : & 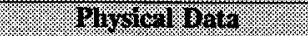 & \\
\hline 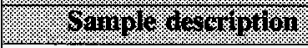 & 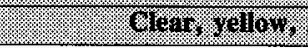 & 6ringin \\
\hline $\mathrm{pH}$ & 11.6 & $\mathrm{n} / \mathrm{a}$ \\
\hline Specific gravity & 1.0056 & $\mathrm{n} / \mathrm{a}$ \\
\hline Water & 96.38 & $\%$ \\
\hline Energetics & no exotherm & $\mathrm{J} / \mathrm{g}$ \\
\hline \multicolumn{3}{|l|}{ Chenvical A ralysio } \\
\hline $\mathrm{OH}$ & $<0.01$ & $M$ \\
\hline $\mathrm{Al}$ & $<7.60 \mathrm{E}-03$ & $M$ \\
\hline $\mathrm{Na}$ & 0.515 & $M$ \\
\hline $\mathrm{NO}_{2}$ & $3.17 \mathrm{E}-03$ & $M$ \\
\hline
\end{tabular}


Table B2-68. 1973 or 1974 Supernatant Sample. ${ }^{1,2}$ (2 sheets)

\begin{tabular}{|c|c|c|}
\hline 1.80 & 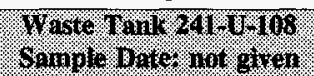 & \\
\hline 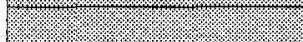 & Samplo Nomber t IG & \\
\hline COMTOMUTI & 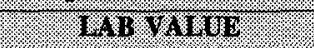 & Tab E.TIT \\
\hline $\mathrm{NO}_{3}$ & 0.381 & $M$ \\
\hline $\mathrm{SO}_{4}$ & $4.93 \mathrm{E}-03$ & $\bar{M}$ \\
\hline $\mathrm{PO}_{4}$ & 0.137 & $\bar{M}$ \\
\hline $\mathrm{F}$ & $1.27 \mathrm{E}-02$ & $M$ \\
\hline $\mathrm{CO}_{3}$ & 0.024 & $M$ \\
\hline 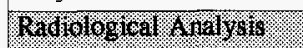 & 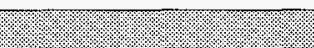 & (2:-1: \\
\hline${ }^{134} \mathrm{Cs}$ & 43.37 & $\mu \mathrm{Ci} / \mathrm{L}$ \\
\hline${ }^{137} \mathrm{Cs}$ & 1,690 & $\mu \mathrm{Ci} / \mathrm{L}$ \\
\hline $\mathrm{Pu}$ & $1.04 \mathrm{E}-06$ & $\mathrm{~g} / \mathrm{L}$ \\
\hline${ }^{89 / 90} \mathrm{Sr}$ & 4.54 & $\mu \mathrm{Ci} / \mathrm{L}$ \\
\hline
\end{tabular}

Notes:

'Pre-1989 analytical data have not been validated and should be used with caution.

${ }^{2}$ Sant (1974) 
HNF-SD-WM-ER-639 Rev. 0

Table B2-69. 1975 Sludge Sample. ${ }^{1,2}$

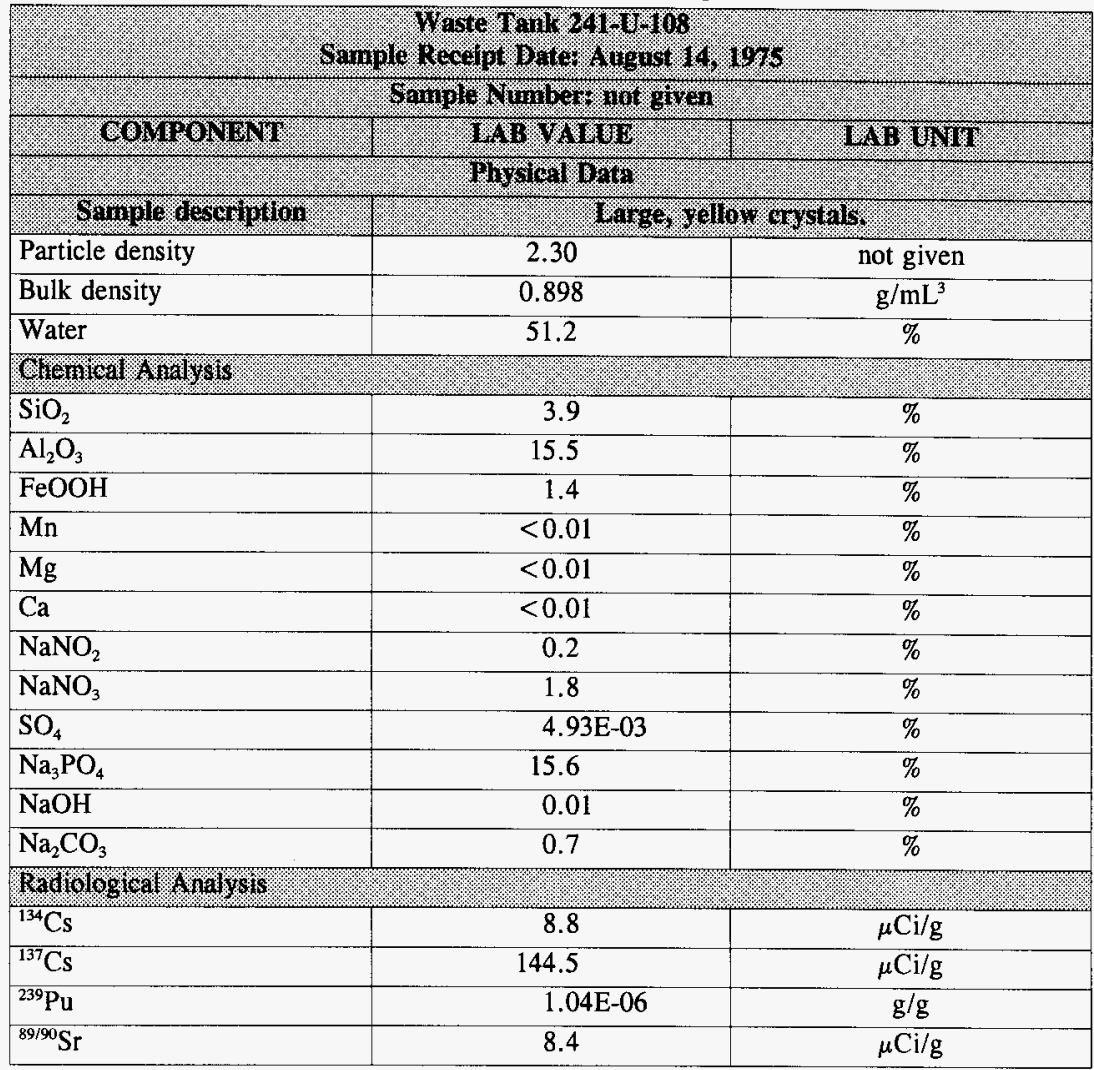

Notes:

'Pre-1989 analytical data have not been validated and should be used with caution.

${ }^{2}$ Horton (1975)

${ }^{3}$ Units were not provided in reference and are assumed to be $\mathrm{g} / \mathrm{mL}$. 


\section{B3.0 ASSESSMENT OF CHARACTERIZATION RESULTS}

The purpose of this chapter is to discuss the overall quality and consistency of the current sampling results for tank $241-\mathrm{U}-108$, and to present the results of the calculation of an analytical-based inventory.

This section also evaluates sampling and analysis factors that may impact interpretation of the data. These factors are used to assess the overall quality and consistency of the data and to identify any limitations in the use of the data.

\section{B3.1 FIELD OBSERVATIONS}

The safety screening, historical, and organic DQO requirements on the 1996 core samples that vertical profiles from at least two widely spaced risers be obtained was not completely fulfilled. Sample from the lower half of segment 2 was only recovered from core sample 146. A sample from each half segment was recovered from at least two of the three cores for all other segments. The sampling requirements of the waste compatibility and vapor DQOs were met. Water with a lithium bromide tracer was used to acquire most of the core samples. There was no evidence of contamination from hydrostatic head fluid or wash water. In addition, all analyses were performed in accordance with the required procedures, with the exception of carbonate which was determined by the TIC method rather than by IC. Carbonate cannot be determined by the IC method prescribed in the core SAP (Homi 1996).

\section{B3.2 QUALITY CONTROL ASSESSMENT}

The usual quality control assessment includes an evaluation of the appropriate standard recoveries, spike recoveries, duplicate analyses, and blanks that are performed in conjunction with the chemical analyses. All the pertinent quality control tests were conducted on the 1996 core and the 1995 grab and vapor samples, allowing a full assessment regarding the accuracy and precision of the data. The respective SAPs (Homi 1996, Schreiber 1995, and Homi 1995) established the specific criteria for all analytes. For the core and grab samples, sample and duplicate pairs that had one or more QC results outside the specified criteria were identified by footnotes in the data summary tables.

The standard and spike recovery results provide an estimate of the accuracy of the analysis. If a standard or spike recovery is above or below the given criterion, the analytical results may be biased high or low, respectively. The precision is estimated by the relative percent difference (RPD), which is defined as the absolute value of the difference between the primary and duplicate samples, divided by their mean, times one hundred. 


\section{B3.2.1 1996 Core Samples}

Occasional fusion preparation blanks showed results above the detection level for some ICP analytes. The level of the respective analyte in a preparation blank was inconsequential when compared to the result for the sample; therefore, the contamination did not impact the sample data quality. Most fusion preparation blanks showed results above the detection level for Sr-90 activity; , however, the contamination was low enough that it did not impact sample data quality.

Tank 241-U-108 core samples were affected by an incorrect spike recovery calculation for the TOC and TIC analyses (Rice 1996). Corrections were made to the TOC and TIC data summary tables (Tables B2-10 and B2-12). However, selected spike recovery data in the tank 241-U-108 data report (Bell 1996a) are not correct. Corrections to Bell (1996a) are due October 30, 1997.

B3.2.1.1 Direct Analyses. The core sample data show that many samples were heterogeneous at the 15 - to 30 -mg level as evidenced by the sample descriptions provided in Section B1.2 and the numerous DSC and TGA RPD values that exceeded the 10 percent core SAP criterion. The sample that exceeded the DSC notification limit was rerun due to the large RPD (45.6) between sample and duplicate results with little improvement (41.4). Triplicate runs were performed on several DSC, TGA, and TOC analyses with poor RPD values between the sample and duplicate results.

The data indicate that the fluctuation of TOC and TIC results from subsegment to subsegment was most likely due to heterogeneity in the samples rather than any analytical problems. Spike recovery and RPD TOC data were within the core SAP limits (80 to 120 percent spike recovery and $\leq 20$ percent RPD) for 51 of 57 subsamples. No re-runs were initiated because the results were far below the $30,000-\mu \mathrm{g} / \mathrm{g}$ notification limit; however, triplicate runs were performed and reported on six samples. For the TIC analyses, the QC criteria of RPD ( $\leq 10$ percent) and spike recovery ( 90 to 110 percent) were tighter and were not always met. The highest RPD value was 31.1 (upper half of segment $3 \mathrm{~A}$, core 146) and the poorest spike recovery was 142 percent (upper half of segment $4 \mathrm{~A}$, core 141).

B3.2.1.2 Inductively Coupled Plasma Analyses. At least three analyses for each of the elements $\mathrm{Al}, \mathrm{Cr}, \mathrm{Fe}, \mathrm{Na}, \mathrm{Ni}, \mathrm{P}$ and $\mathrm{Si}$ had $\mathrm{RPD}$ values and/or spike recoveries outside of the core SAP limit of $<=10$ percent for the RPD and 90 to 110 percent for the spike recovery. Because of the high concentrations of sodium in all samples and aluminum in the drainable liquid and water-digested samples, the poor spike recoveries associated with these samples were reported, but not evaluated. The acid-digested core composite samples analyzed for Si had low spike recoveries. This was most likely caused by the sample result being biased high because of Si leaching from the laboratory glassware.

As with the TOC and TIC results, the poor RPD values and spike recoveries were attributed to sample heterogeneity. Two fusion digests were re-run because of poor precision between the sample and duplicate results. 
B3.2.1.3 Ion Chromatography Analyses. Quality control issues are discussed for the bromide and nitrate determinations. Additional IC data were collected on an opportunistic basis and are reported here. Because these data were not identified in any DQO document, there were no programmatic QC requirements with respect to these data and the QC information associated with them were not evaluated for this report.

Bromide was only detected in the field blank; therefore, there were no exceptions to the QC parameters stated in the SAP for the samples analyzed for bromide. For nitrate, however, eight of 51 samples had RPD values in excess of the required 10 percent, and ten of the samples had spike recoveries outside the SAP 90-110 percent limit. The poor RPD values nd spike recoveries were attributed to sample heterogeneity.

B3.2.1.4 Radiochemical Analyses. Quality control issues are discussed for the total alpha, Cs-137 (by GEA), Sr-90, total uranium, and total beta determinations. Additional GEA data were collected on an opportunistic basis and are reported here. Because these data were not identified in any DQO document, there were no programmatic QC requirements with respect to these data and the QC information associated with them were not evaluated for this report.

For the total alpha activity analyses, the core SAP criterion for RPD between sample and duplicate results ( $\leq 10$ percent) was exceeded in 27 samples, and the core SAP criterion for spike recovery (90-110 percent) was exceeded on 18 samples. The samples that exceeded the RPD and/or spike recovery criteria probably did so due to sample heterogeneity and a high solids content.

One sample measured for Cs-137 activity (core 146 composite) had an RPD value of 43.2 , which exceeded the core SAP limit of 20 percent. Six of 53 fusion digest samples measured for $\mathrm{Sr}-90$ activity had RPD values greater than 10 percent as specified in the core SAP. These RPD values ranged from 11.3 to 46.9 percent. The sample with the RPD value of 46.9 (core 145 composite) had been re-run, as had three other samples.

The initial total uranium sample and duplicate results from core 145 had an RPD that exceeded the SAP criterion of $<20$ percent. The sample was re-run with a satisfactory RPD result, but with a low spike recovery (48.3 percent).

The initial sample and duplicate total beta activity results from core 145 had an RPD which exceeded the core SAP criterion of $<20$ percent. At 44.8 , the rerun RPD results still did not meet the SAP RPD criterion.

\section{B3.2.2 1995 Grab Samples}

All QC criteria were met for the 1995 grab sample analyses on sample U-108-1. 
In summary, the vast majority of the QC results were within the boundaries specified in the core and grab SAPs. The discrepancies mentioned here and footnoted in the data summary tables in Appendix B should not impact the validity or use of the data.

\section{B3.3 DATA CONSISTENCY CHECKS}

Comparisons of different analytical methods can help to assess the consistency and quality of the data. Several comparisons were possible with the data set provided by the three core samples. These include a comparison of phosphorous and sulfur as analyzed by ICP with phosphate and sulfate as analyzed by IC, and a comparison of TOC concentration as determined by the persulfate and furnace oxidation methods. In addition, mass and charge balances were calculated and a comparison of calculated total beta activity and measured total beta activity was made. A comparison of 1996 core drainable liquid and 1995 grab sample results helped assess the overall data consistency.

\section{B3.3.1 Comparison of Results from Different Analytical Methods}

The following data consistency checks compare the results from two different analytical methods. Agreement between the two methods strengthens the credibility of both results, whereas poor agreement brings the reliability of the data into question. All analytical mean results were taken from Table B3.6.

B3.3.1.1 Solid Core Samples. Phosphate and sulfate data were measured by IC, and phosphorous and sulfur were measured by ICP, which allows a comparison of the IC and ICP results. The mean phosphorous core composite result on the water-digested sample was $4,000 \mu \mathrm{g} / \mathrm{g}$. Surprisingly, this result was higher than the results on the acid-digested and fusion-digested samples and was the one used for comparison to the (water-digested) IC phosphate result. The concentration of phosphorous found converts to $12,264 \mu \mathrm{g} / \mathrm{g}$ of phosphate, and compares very well with the IC result of $12,400 \mu \mathrm{g} / \mathrm{g}$.

The mean sulfur core composite result on the water-digested sample was $5,530 \mu \mathrm{g} / \mathrm{g}$. This result was slightly higher than the acid-digested result and was the one used for comparison to the (water-digested) IC sulfate result. The concentration of sulfur found converts to $16,570 \mu \mathrm{g} / \mathrm{g}$ of sulfate, which compares very well with the IC result of $17,600 \mu \mathrm{g} / \mathrm{g}$.

B3.3.1.2 Drainable Liquid Samples. Phosphate and phosphorous, and sulfate and sulfur concentrations were also measured on the drainable liquid samples from segment 1 of cores 141,145 , and 146. The mean ICP phosphorous result was $1,020 \mu \mathrm{g} / \mathrm{mL}$, which converts to $3,130 \mu \mathrm{g} / \mathrm{mL}$ of phosphate. The phosphate result was $2,500 \mu \mathrm{g} / \mathrm{mL}$, which accounts for approximately 80 percent of the phosphorous. The sulfur/sulfate comparison on the drainable liquids was more favorable. The mean ICP sulfur result was $1,410 \mu \mathrm{g} / \mathrm{mL}$, which converts to $4,220 \mu \mathrm{g} / \mathrm{mL}$ of sulfate. The observed IC sulfate value was $3,630 \mu \mathrm{g} / \mathrm{mL}$; accounting for 86 percent of the sulfur. 


\section{B3.3.2 Mass and Charge Balance}

The principle objective in performing mass and charge balances is to determine if the measurements are self-consistent. In calculating the balances, only analytes listed in Table B3-2 detected at a concentration of $1,000 \mu \mathrm{g} / \mathrm{g}$ or greater were considered.

Except for sodium, all cations listed in Table B3-1 were assumed to be in their most common oxide form, and the concentrations of the assumed species were calculated stoichiometrically. The aluminum concentration was assumed to be present as the aluminate ion because of the high concentration of aluminum found in the drainable liquid and water-digested samples. Because precipitates are neutral species, all positive charge was attributed to the sodium cation. The anions listed in Table B3-2 were assumed to be present as sodium salts and were expected to balance the positive charge exhibited by the cations. Phosphate, as determined by IC, is assumed to be completely water soluble and appears only in the anion mass and charge calculations. The concentrations of cationic species in Table B3-1, the anionic species in Table B3-2, and the percent water were ultimately used to calculate the mass balance.

Table B3-1. Cation Mass and Charge Data.

\begin{tabular}{|c|c|c|c|c|}
\hline Analye & Gancentration & $\begin{array}{l}\text { Arrumed } \\
\text { Species }\end{array}$ & 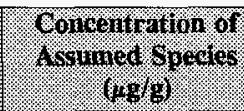 & $\begin{array}{l}\text { chinge } \\
\text { (peorg) }\end{array}$ \\
\hline Sodium & 241,000 & $\mathrm{Na}^{+}$ & 241,000 & 10,480 \\
\hline
\end{tabular}


HNF-SD-WM-ER-639 Rev. 0

Table B3-2. Anion and Neutral Species Mass and Charge Data.

\begin{tabular}{|c|c|c|c|c|}
\hline \multirow[b]{2}{*}{ Analyte } & Eoneritrintion & \multirow{2}{*}{ 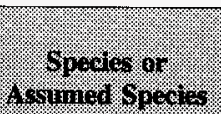 } & 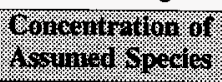 & \multirow{2}{*}{$\frac{\text { Charge }}{\text { requg }}$} \\
\hline & (1.96. & & $4=196$ : & \\
\hline Aluminum & 16,500 & $\mathrm{AlO}_{2}^{-}$ & 36,100 & 612 \\
\hline TOC & 4,160 & $\mathrm{C}_{2} \mathrm{O}_{4}{ }^{2-}$ & 15,300 & 347 \\
\hline TIC & 7,970 & $\mathrm{CO}_{3}{ }^{2-}$ & 39,800 & 1,330 \\
\hline Chromium & 4,120 & $\mathrm{Cr}(\mathrm{OH})_{3}$ & 8,160 & $\mathrm{n} / \mathbf{a}$ \\
\hline Chloride & 3,450 & $\mathrm{Cl}^{-}$ & 3,450 & 97 \\
\hline Nitrate & 295,000 & $\mathrm{NO}_{3}^{-}$ & 295,000 & 4,760 \\
\hline Nitrite & 50,000 & $\mathrm{NO}_{2}^{-}$ & 50,000 & 1,090 \\
\hline Phosphate & 17,200 & $\mathrm{PO}_{4}^{3-}$ & 17,200 & 542 \\
\hline Sulfate & 15,100 & $\mathrm{SO}_{4}{ }^{2-}$ & 15,100 & 314 \\
\hline Total & & & 480,100 & 9,090 \\
\hline
\end{tabular}

The mass balance was calculated from the formula below. The factor 0.0001 is the conversion factor from $\mu \mathrm{g} / \mathrm{g}$ to weight percent.

Mass balance $=\%$ Water $+0.0001 \times$ \{Total Analyte Concentration\}

$$
\begin{gathered}
\% \text { water }+0.0001 \times\left\{\left[\mathrm{Na}^{+}\right]+\left[\mathrm{AlO}_{2}\right]+\left[\mathrm{C}_{2} \mathrm{O}_{4}{ }^{2-}\right]+\left[\mathrm{CO}_{3}{ }^{2}\right]+\right. \\
\left.\mathrm{Cr}(\mathrm{OH})_{3}+[\mathrm{Cl}]+\left[\mathrm{NO}_{3}{ }^{-}\right]+\left[\mathrm{NO}_{2}\right]+\left[\mathrm{PO}_{4}{ }^{3-}\right]+\left[\mathrm{SO}_{4}{ }^{2-}\right]\right\}
\end{gathered}
$$

The mass balance resulting from adding the percent water to the total analyte concentration is 108.3 percent as shown in Table B3-3.

\begin{tabular}{|c|c|}
\hline (1) Total & Concentringing \\
\hline Total from Table B3-1 & 241,000 \\
\hline Total from Table B3-2 & 480,100 \\
\hline Water \% & 364,000 \\
\hline Grand total & $1,085,100$ \\
\hline
\end{tabular}

Table B3-3. Mass Balance Totals. 
The following equations demonstrate the derivation of total cations and total anions; the charge balance is the ratio of these two values.

Total cations $(\mu \mathrm{eq} / \mathrm{g})=\left[\mathrm{Na}^{+}\right] / 23.0=10,480 \mu \mathrm{eq} / \mathrm{g}$

Total anions $(\mu \mathrm{eq} / \mathrm{g})=\left[\mathrm{AlO}_{2}{ }^{-}\right] / 59.0+\left[\mathrm{C}_{2} \mathrm{O}_{4}{ }^{2-}\right] / 44.0+\left[\mathrm{CO}_{3}{ }^{2-}\right] / 30.0+\left[\mathrm{Cl}^{-}\right] / 35.5+$ $\left[\mathrm{NO}_{3}{ }^{-}\right] / 62.0+\left[\mathrm{NO}_{2}{ }^{-}\right] / 46.0+\left[\mathrm{PO}_{4}{ }^{3-}\right] / 31.7+\left[\mathrm{SO}_{4}{ }^{2-}\right] / 48.1=9,090 \mu \mathrm{eq} / \mathrm{g}$

The charge balance obtained by dividing the sum of the positive charge by the sum of the negative charge was 1.15 .

\section{B3.3.3 Total Beta Results}

The mean total beta result obtained from the core composite data was compared with the measured beta emitters ${ }^{137} \mathrm{Cs}$ and ${ }^{89 / 90} \mathrm{Sr}$. For consistency, all results used were the mean core composite data shown in Table B3-4.

Total beta $($ calculated $)=\left[{ }^{137} \mathrm{Cs}\right]+(2)\left(\left[{ }^{89 / 90} \mathrm{Sr}\right]\right)=162 \mu \mathrm{Ci} / \mathrm{g}+(2)(9.32)=181 \mu \mathrm{Ci} / \mathrm{g}$.

The factor of two arises from the fact that ${ }^{90} \mathrm{Sr}$ is in equilibrium with ${ }^{90} \mathrm{Y}$, which has a much shorter half life. Thus, for every beta decay from strontium, there is an additional beta decay from the yttrium.

The measured mean total beta activity on the core composites was $167 \mu \mathrm{Ci} / \mathrm{g}$.

In summary, the above calculations yield results $<=15$ percent of the theoretical values, indicating that the analytical results are generally self-consistent.

\section{B3.3.4 Comparison of 1996 Core Drainable Liquid and 1995 Grab Sample Results}

Because no waste transfers were made in or out of tank 241-U-108 between the collection of the 1995 grab samples and the 1996 core samples, the analytical results for the (liquid) grab samples and the drainable liquids should be similar. Recall that all drainable liquid was collected from the first segment of the three core samples. The results are taken from the tables in Section B2.0. 


\section{B3.3.5 Comparison of TOC by Persulfate and TOC by Furnace Oxidation}

As shown in Table B3-4, there is generally good agreement between the 1995 liquid grab samples and what is expected to be the same liquid obtained from the 1996 core sampling. This agreement allows for a comparison between the two TOC methods available at the 222-S Laboratory: furnace oxidation and persulfate oxidation. The furnace oxidation method has been shown (Baldwin et al. 1994) to be effective at oxidizing hydrocarbons such as dodecane, while the persulfate method is not. The TOC content of the 1995 liquid grab sample was measured at $7,440 \mu \mathrm{g} / \mathrm{mL}$ by furnace oxidation. The TOC content of the 1996 drainable liquid samples averaged $7,160 \mu \mathrm{g} / \mathrm{mL}$, indicating that there is little or no organic carbon that was not oxidized by the persulfate method.

\section{B3.3.6 Comparison of Transfer History and Analytical Results}

The HTCE of selected tank 241-U-108 analytes appears in Table B3-5 along with the concentration estimates from the core sample analytical results. This comparison is presented for information purposes only. The HTCE values are generated from a combination of inputs: transfer history, estimated compositions of various Hanford Site waste types, and modeled waste layering (see Agnew et al. 1996a). Each of the three inputs contains assumptions and/or other factors (such as transfers of an unknown waste type into the tank) that may impact HTCE data. Because of uncertainty of waste volumes and the fact that the HTCE values have not been validated, they should be used with caution.

Comparing the HTCE with the most recent analytical values produced varied results, yet some analytes such as bulk density, water, potassium, and nitrate compared quite favorably. The largest disparity was found with oxalate values. The HTCE value does not consider the TOC degradation that is probably responsible for the observed oxalate concentration. The discrepancy in the uranium numbers most likely results from the fact that MW was not recovered from the tank, although the HTCE model considers there to be metal waste in the tank. Agnew et al. (1996a) is believed to have overestimated the amount of cladding waste in the tank, which would account for the overestimation of aluminum, carbonate, and uranium. The reasons for the overestimation of the sulfate and strontium concentrations may be related to inaccurate source term assumptions about the waste composition.

\section{B3.4 MEAN CONCENTRATIONS AND CONFIDENCE INTERVALS}

The following evaluation was performed on the analytical data from the April/May 1995 core samples for tank 241-U-108. Analysis of variance (ANOVA) models were fit to the 1996 core samples from tank 241-U-108. These models were used to compute an estimate of mean concentration, the variance of the mean, and 95 percent confidence intervals on the mean for each detected analyte. 
Table B3-4. Comparison of 1995 Grab and 1996 Drainable Liquid Analytical Results.

\begin{tabular}{|c|c|c|}
\hline Anolite & 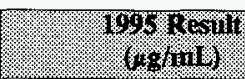 & 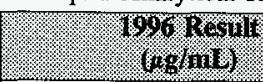 \\
\hline Al & 40,170 & 40,700 \\
\hline $\mathrm{Fe}$ & $\overline{40.1}$ & 33.3 \\
\hline $\mathrm{Na}$ & 288,100 & 243,000 \\
\hline Chloride & 8,415 & 8,940 \\
\hline Fluoride & 1,120 & $790^{1}$ \\
\hline Nitrate & 173,500 & 197,000 \\
\hline Nitrite & 115,500 & 145,000 \\
\hline Phosphate & $3,700^{2}$ & 2,500 \\
\hline Sulfate & 4,595 & 3,630 \\
\hline Water & $50.27 \%$ & $49.93 \%$ \\
\hline TIC & 3,210 & 4,400 \\
\hline
\end{tabular}

Notes:

'Fluoride was only detected in the core 146 drainable liquid.

${ }^{2}$ The duplicate result was a less-than value.

Table B3-5. Comparison of Historical Tank Content Estimate ${ }^{1}$ with 1996 Core Sample Analytical Results from Tank 241-U-108. (2 sheets)

\begin{tabular}{|c|c|c|}
\hline Analle: & 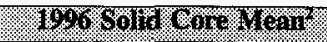 & HIT O Fstinate \\
\hline 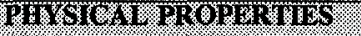 & 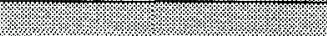 & 药 \\
\hline Percent water (TGA) & $31.5 \%$ & $33.0 \%$ \\
\hline Bulk density & $1.71 \mathrm{~g} / \mathrm{mL}$ & $1.62 \mathrm{~g} / \mathrm{mL}$ \\
\hline MUWHS: & 1. $48 \mathrm{~g}$ & $14 \mathrm{ge}$ \\
\hline Aluminum & 16,500 & 33,600 \\
\hline Chromium & 4,120 & 2,080 \\
\hline Potassium & $1,580^{3}$ & 1,420 \\
\hline Sodium & $2.41 \mathrm{E}+\overline{05}$ & $1.78 \mathrm{E}+05$ \\
\hline AMIONS : & 19: & ln: \\
\hline Carbonate (TIC) & 4,400 & 19,100 \\
\hline Chloride & 8,940 & 4,780 \\
\hline Nitrate & $1.81 \mathrm{E}+05$ & $2.06 \mathrm{E}+05$ \\
\hline Nitrite & $1.30 \mathrm{E}+05$ & 73,100 \\
\hline Oxalate & $4,640^{3}$ & 1.96 \\
\hline Phosphate & 2,500 & 5,840 \\
\hline Sulfate & 3,630 & 15,600 \\
\hline TOMM U FARON & $1.16 \mathrm{~g}$ & 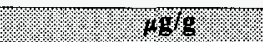 \\
\hline Total organic carbon & 7,160 & 9,240 \\
\hline
\end{tabular}


Table B3-5. Comparison of Historical Tank Content Estimate ${ }^{1}$ with 1996 Core Sample Analytical Results from Tank 241-U-108. (2 sheets)

\begin{tabular}{|c|c|c|}
\hline Mnire & 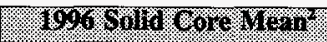 & 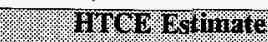 \\
\hline 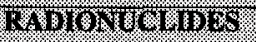 & S & $\sqrt{4} \times 1 / 3$ \\
\hline${ }^{139} \mathrm{Cs}$ & 166 & 117 \\
\hline Total uranium & $187^{3}(\mu \mathrm{g} / \mathrm{g})$ & $7,770(\mu \mathrm{g} / \mathrm{g})$ \\
\hline${ }^{90} \mathrm{Sr}$ & 10.1 & 84.3 \\
\hline
\end{tabular}

Notes:

${ }^{1}$ Agnew et al. (1996b)

${ }^{2}$ From Table B3-5

${ }^{3}$ From Table B3-4

Because an inventory estimate is needed without comparing it to a threshold value, two-sided 95 percent confidence intervals on the mean inventory are computed. These confidence intervals were done with both the composite-level and segment-level data. With segment-level data, the supernatant sample data and solid sample data were analyzed separately. Supernatant samples were only present in segment 1 of both cores.

The upper and lower limits (UL and LL) to a two-sided 95 percent confidence interval for the mean are

$$
\hat{\mu} \pm \mathrm{t}_{(\mathrm{df}, 0.025)} \times \hat{\sigma}_{\hat{\mu}} \text {. }
$$

In these equations, $\hat{\mu}$ is the estimate of the mean concentration, $\hat{\sigma}_{\hat{\mu}}$ is the estimate of the standard deviation of the mean concentration, and $t_{\mathrm{df}, 0.025)}$ is the quantile from Student's $t$ distribution with degrees of freedom (df) for a two-sided 95 percent confidence interval.

The mean, $\hat{\mu}$, and the standard deviation, $\hat{\sigma}_{\hat{\mu}}$, were estimated using restricted maximum likelihood estimation (REML) methods. The degrees of freedom (df), for tank 241-U-108, is the number of cores sampled minus one.

\section{B3.4.1 Composite, Solid Segment, and Liquid Segment Means}

The statistics in this section were based on analytical data from the most recent sampling event of tank 241-U-108. ANOVA techniques were used to estimate the mean, and calculate confidence limits on the mean, for all analytes that had at least 50 percent of reported values above the detection limit. If at least 50 percent of the reported values were above the detection limit, all of the data were used in the computations. The detection limit was used as the value for nondetected results. No ANOVA estimates were computed for analytes with less than 50 percent detected values. Only arithmetic means were computed for these analytes. 
The results given below are ANOVA estimates based on the core composite data from cores 141,145 , and 146 of tank 241-U-108. Estimates of the mean concentration, the standard deviation of the mean, the degrees of freedom, and confidence interval on the mean concentration, are given in Table B3-6. The LL to a 95 percent confidence interval can be negative. Because an actual concentration of less than zero is not possible, the lower limit is reported as zero, whenever this occurred.

Table B3-6. 95 Percent Two-Sided Confidence Interval for the Mean Concentration for Composite Sample Data. (4 sheets)

\begin{tabular}{|c|c|c|c|c|c|c|}
\hline Mnarte & Whis & 1. & to. & dif & XI. & $\sqrt{612}$ \\
\hline ICP.a.Al & $\mu \mathrm{g} / \mathrm{g}$ & $1.84 \mathrm{E}+04$ & $3.77 \mathrm{E}+03$ & 2 & $2.21 \mathrm{E}+03$ & $3.46 \mathrm{E}+04$ \\
\hline ICP.f.Al & $\mu \mathrm{g} / \mathrm{g}$ & $1.88 \mathrm{E}+04$ & $3.17 \mathrm{E}+03$ & 2 & $5.16 \overline{\mathrm{E}+03}$ & $3.25 \mathrm{E}+04$ \\
\hline ICP.w.Al & $\mu \mathrm{g} / \mathrm{g}$ & $1.65 \mathrm{E}+04$ & $2.50 \mathrm{E}+03$ & 2 & $5 . \overline{73 E+03}$ & $2.73 \mathrm{E}+04$ \\
\hline $\mathrm{Am}-241^{1}$ & $\mu \mathrm{Ci} / \mathrm{g}$ & $8.19 \mathrm{E}-01$ & $\mathrm{n} / \mathrm{a}$ & $\mathrm{n} / \mathrm{a}$ & $\mathrm{n} / \mathrm{a}$ & $\mathrm{n} / \mathrm{a}$ \\
\hline$\overline{\text { ICP.a.Sb }}^{\overline{1}}$ & $\mu \mathrm{g} / \mathrm{g}$ & $5.2 \overline{1 \mathrm{E}+01}$ & $n / a$ & $\mathrm{n} / \mathrm{a}$ & $\mathrm{n} / \mathrm{a}$ & $\mathrm{n} / \mathrm{a}$ \\
\hline ICP.w. $\mathrm{Sb}^{1}$ & $\mu \mathrm{g} / \mathrm{g}$ & $4.89 \mathrm{E}+01$ & $\mathrm{n} / \mathrm{a}$ & $\mathrm{n} / \mathrm{a}$ & $\mathrm{n} / \mathrm{a}$ & $\mathrm{n} / \mathrm{a}$ \\
\hline ICP.a.As ${ }^{1}$ & $\mu \mathrm{g} / \mathrm{g}$ & $8.14 \mathrm{E}+01$ & $n / a$ & $\mathrm{n} / \mathrm{a}$ & $\mathrm{n} / \mathrm{a}$ & $n / a$ \\
\hline ICP.W.As ${ }^{1}$ & $\mu \mathrm{g} / \mathrm{g}$ & $8.15 \mathrm{E}+01$ & $\mathrm{n} / \mathrm{a}$ & $\mathrm{n} / \mathrm{a}$ & $\mathrm{n} / \mathrm{a}$ & $\mathrm{n} / \mathrm{a}$ \\
\hline ICP.a.Ba ${ }^{1}$ & $\mu \mathrm{g} / \mathrm{g}$ & $4.07 \mathrm{E}+01$ & $\mathrm{n} / \mathrm{a}$ & $\mathrm{n} / \mathrm{a}$ & $\mathrm{n} / \mathrm{a}$ & $\mathrm{n} / \mathrm{a}$ \\
\hline ICP.w.Ba ${ }^{1}$ & $\mu \mathrm{g} / \mathrm{g}$ & $4.07 \mathrm{E}+01$ & $\mathrm{n} / \mathrm{a}$ & $\mathrm{n} / \mathrm{a}$ & $\mathrm{n} / \mathrm{a}$ & $\mathrm{n} / \mathrm{a}$ \\
\hline$\overline{\mathrm{I}} \overline{\mathrm{CP}} \cdot \mathrm{a} \cdot \mathrm{Be}^{\mathrm{I}}$ & $\mu \mathrm{g} / \mathrm{g}$ & $4.07 \mathrm{E}+00$ & $\mathrm{n} / \mathrm{a}$ & $n / a$ & $\mathrm{n} / \mathrm{a}$ & $\mathrm{n} / \mathrm{a}$ \\
\hline ICP.w.Be & $\mu \mathrm{g} / \mathrm{g}$ & $4.07 \mathrm{E}+00$ & $\mathrm{n} / \mathrm{a}$ & $\mathrm{n} / \mathrm{a}$ & $n / a$ & $\mathrm{n} / \mathrm{a}$ \\
\hline ICP.a.Bi ${ }^{1}$ & $\mu \mathrm{g} / \mathrm{g}$ & $8.14 \mathrm{E}+01$ & $n / a$ & $\mathrm{n} / \mathrm{a}$ & $\mathrm{n} / \mathrm{a}$ & $n / a$ \\
\hline ICP.f.Bi & $\mu \mathrm{g} / \mathrm{g}$ & $1.92 \mathrm{E}+03$ & $\mathrm{n} / \mathrm{a}$ & $\mathrm{n} / \mathrm{a}$ & $\mathrm{n} / \mathrm{a}$ & $\bar{n} / \mathbf{a}$ \\
\hline ICP.w.Bi ${ }^{1}$ & $\mu \mathrm{g} / \mathrm{g}$ & $8.15 \mathrm{E}+01$ & $\mathrm{n} / \mathrm{a}$ & $\mathrm{n} / \mathrm{a}$ & $\mathrm{n} / \mathrm{a}$ & $\mathrm{n} / \mathrm{a}$ \\
\hline ICP.a.B & $\mu \mathrm{g} / \mathrm{g}$ & $8.04 \mathrm{E}+01$ & $1.56 \bar{E}+01$ & 2 & $1.31 \mathrm{E}+01$ & $1.48 \mathrm{E}+02$ \\
\hline$\overline{\mathrm{ICP}} \cdot \overline{\mathrm{w} . \mathrm{B}}$ & $\mu \mathrm{g} / \mathrm{g}$ & $5.24 \mathrm{E}+02$ & $2.85 \mathrm{E}+01$ & 2 & $4.02 \mathrm{E}+02$ & $6.47 \mathrm{E}+02$ \\
\hline Bromide $^{1}$ & $\mu \mathrm{g} / \mathrm{g}$ & $6.23 \mathrm{E}+02$ & $\mathrm{n} / \mathrm{a}$ & $\mathrm{n} / \mathrm{a}$ & $n / a$ & $\mathrm{n} / \mathrm{a}$ \\
\hline $\begin{array}{l}\text { Bulk } \\
\text { density }\end{array}$ & $\mathrm{g} / \mathrm{mL}$ & $1.71 \mathrm{E}+00$ & $3.93 \mathrm{E}-02$ & 2 & $1.54 \mathrm{E}+00$ & $1.88 \mathrm{E}+00$ \\
\hline$\overline{\mathrm{ICP} . \mathrm{a} . \mathrm{Cd}^{2}}$ & $\mu \mathrm{g} / \mathrm{g}$ & $4.46 \mathrm{E}+00$ & $4.76 \mathrm{E}-01$ & 2 & $2.41 \mathrm{E}+00$ & $6.51 \mathrm{E}+00$ \\
\hline$\overline{\text { ICP.w. }} \cdot \overline{\mathrm{Cd}^{1}}$ & $\mu \mathrm{g} / \mathrm{g}$ & $4.07 \mathrm{E}+00$ & $\mathrm{n} / \mathbf{a}$ & $n / a$ & $\mathrm{n} / \mathrm{a}$ & $\mathrm{n} / \mathrm{a}$ \\
\hline ICP.a.Ca & $\mu \mathrm{g} / \mathrm{g}$ & $1.56 \mathrm{E}+02$ & $1.82 \mathrm{E}+01$ & 2 & $7.71 \mathrm{E}+01$ & $2.34 \mathrm{E}+02$ \\
\hline ICP.f.Ca & $\mu \mathrm{g} / \mathrm{g}$ & $1.92 \mathrm{E}+03$ & $\mathrm{n} / \mathrm{a}$ & $\mathrm{n} / \mathrm{a}$ & $\mathrm{n} / \mathrm{a}$ & $n / a$ \\
\hline ICP.w.Ca & $\mu \mathrm{g} / \mathrm{g}$ & $1.15 \mathrm{E}+02$ & $1.58 \mathrm{E}+01$ & 2 & $4 . \overline{72 E+01}$ & $1.83 \mathrm{E}+02$ \\
\hline ICP.a.Ce ${ }^{1}$ & $\mu \mathrm{g} / \mathrm{g}$ & $8.14 \mathrm{E}+01$ & $\mathrm{n} / \mathrm{a}$ & $\mathrm{n} / \mathrm{a}$ & $\mathrm{n} / \mathrm{a}$ & $\mathrm{n} / \mathrm{a}$ \\
\hline ICP.w.Ce ${ }^{1}$ & $\mu \mathrm{g} / \mathrm{g}$ & $8.15 \mathrm{E}+01$ & $\mathrm{n} / \mathrm{a}$ & $\mathrm{n} / \mathrm{a}$ & $\mathrm{n} / \mathrm{a}$ & $\mathrm{n} / \mathrm{a}$ \\
\hline Cs-137 & $\mu \mathrm{Ci} / \mathrm{g}$ & $1.62 \mathrm{E}+02$ & $1.39 \mathrm{E}+01$ & 2 & $1.03 \mathrm{E}+02$ & $2.22 \mathrm{E}+02$ \\
\hline Chloride & $\mu \mathrm{g} / \mathrm{g}$ & $4.38 \mathrm{E}+03$ & $6.72 \mathrm{E}+02$ & 2 & $1 . \overline{49 \mathrm{E}+03}$ & $7.27 \widehat{\mathrm{E}+03}$ \\
\hline
\end{tabular}


Table B3-6. 95 Percent Two-Sided Confidence Interval for the Mean Concentration for Composite Sample Data. (4 sheets)

\begin{tabular}{|c|c|c|c|c|c|c|}
\hline 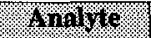 & Soins & \%. & 6 & iff & 16 & OU. \\
\hline ICP.a.Cr & $\mu \mathrm{g} / \mathrm{g}$ & $3.83 \mathrm{E}+03$ & $1.33 \mathrm{E}+02$ & 2 & $3.25 \mathrm{E}+03$ & $4.40 \mathrm{E}+03$ \\
\hline ICP.f.Cr & $\mu \mathrm{g} / \mathrm{g}$ & $4.06 \mathrm{E}+03$ & $2.55 \mathrm{E}+02$ & 2 & $2.96 \mathrm{E}+03$ & $5.16 \mathrm{E}+03$ \\
\hline ICP.w.Cr & $\mu \mathrm{g} / \mathrm{g}$ & $3.56 \mathrm{E}+02$ & $5.73 \mathrm{E}+01$ & 2 & $1.09 E+02$ & $6.02 \mathrm{E}+02$ \\
\hline ICP.a.Co ${ }^{1}$ & $\mu \mathrm{g} / \mathrm{g}$ & $1.63 \mathrm{E}+01$ & $\mathrm{n} / \mathrm{a}$ & $\mathrm{n} / \mathrm{a}$ & $\mathrm{n} / \mathrm{a}$ & $\mathrm{n} / \mathrm{a}$ \\
\hline ICP.w.Co' & $\mu \mathrm{g} / \mathrm{g}$ & $1.63 \mathrm{E}+01$ & $\mathrm{n} / \mathrm{a}$ & $\mathrm{n} / \mathrm{a}$ & $\mathrm{n} / \mathrm{a}$ & $\mathrm{n} / \mathrm{a}$ \\
\hline Co-60 & $\mu \mathrm{Ci} / \mathrm{g}$ & $2.04 \mathrm{E}-02$ & $\mathrm{n} / \mathrm{a}$ & $\mathrm{n} / \mathrm{a}$ & $\mathrm{n} / \mathrm{a}$ & $n / a$ \\
\hline ICP.a. $\mathrm{Cu}^{1}$ & $\mu \mathrm{g} / \mathrm{g}$ & $8.30 \mathrm{E}+00$ & $\mathrm{n} / \mathrm{a}$ & $\mathrm{n} / \mathrm{a}$ & $n / a$ & $\bar{n} / \mathrm{a}$ \\
\hline ICP.w.Cu ${ }^{1}$ & $\mu \mathrm{g} / \mathrm{g}$ & $8.15 \mathrm{E}+00$ & $\mathrm{n} / \mathrm{a}$ & $\mathrm{n} / \mathrm{a}$ & $\mathrm{n} / \mathrm{a}$ & $\mathrm{n} / \mathrm{a}$ \\
\hline $\mathrm{Eu}-\overline{154^{1}}$ & $\mu \mathrm{Ci} / \mathrm{g}$ & $7.22 \mathrm{E}-02$ & $\mathrm{n} / \mathrm{a}$ & $\mathrm{n} / \mathrm{a}$ & $\mathrm{n} / \mathrm{a}$ & $\mathrm{n} / \mathrm{a}$ \\
\hline Eu-155 & $\mu \overline{\mathrm{Ci} / \mathrm{g}}$ & $3.66 \mathrm{E}-01$ & $\mathrm{n} / \mathrm{a}$ & $\mathrm{n} / \mathrm{a}$ & $\mathrm{n} / \mathrm{a}$ & $\mathrm{n} / \mathrm{a}$ \\
\hline Fluoride $^{1}$ & $\mu \mathrm{g} / \mathrm{g}$ & $2.38 \mathrm{E}+02$ & $\mathrm{n} / \mathrm{a}$ & $\mathrm{n} / \mathrm{a}$ & $\mathrm{n} / \mathrm{a}$ & $\mathrm{n} / \mathrm{a}$ \\
\hline Alpha & $\mu \mathrm{Ci} / \mathrm{g}$ & $4.40 \mathrm{E}-02$ & $5.91 \mathrm{E}-03$ & 2 & $1.86 \mathrm{E}-02$ & $6.9 \overline{4 E}-02$ \\
\hline Beta & $\mu \mathrm{Ci} / \mathrm{g}$ & $1.67 \mathrm{E}+02$ & $1.06 \mathrm{E}+01$ & 2 & $1.21 \mathrm{E}+02$ & $2.12 \mathrm{E}+02$ \\
\hline ICP.a.Fe & $\mu \mathrm{g} / \mathrm{g}$ & $1.75 \mathrm{E}+02$ & $1.59 \mathrm{E}+01$ & 2 & $1.07 \mathrm{E}+02$ & $2.44 \mathrm{E}+02$ \\
\hline ICP.f.Fe ${ }^{1}$ & $\mu \mathrm{g} / \mathrm{g}$ & $9.59 \mathrm{E}+02$ & $\mathrm{n} / \mathrm{a}$ & $\mathrm{n} / \mathrm{a}$ & $n / a$ & $\mathrm{n} / \mathrm{a}$ \\
\hline ICP.w.Fe & $\mu \mathrm{g} / \mathrm{g}$ & $4.07 \mathrm{E}+01$ & $\mathrm{n} / \mathrm{a}$ & $\mathrm{n} / \mathrm{a}$ & $\mathrm{n} / \mathrm{a}$ & $n / a$ \\
\hline ICP.a. $\mathrm{La}^{1}$ & $\mu \mathrm{g} / \mathrm{g}$ & $4.07 \mathrm{E}+01$ & $\mathrm{n} / \mathrm{a}$ & $\mathrm{n} / \mathrm{a}$ & $n / a$ & $\mathrm{n} / \mathrm{a}$ \\
\hline ICP.w. $\mathrm{La}^{1}$ & $\mu \mathrm{g} / \mathrm{g}$ & $4.07 \mathrm{E}+01$ & $\mathrm{n} / \mathrm{a}$ & $\mathrm{n} / \mathrm{a}$ & $n / a$ & $n / a$ \\
\hline ICP.a.Pb ${ }^{1}$ & $\mu \mathrm{g} / \mathrm{g}$ & $8.14 \mathrm{E}+01$ & $\mathrm{n} / \mathrm{a}$ & $\mathrm{n} / \mathrm{a}$ & $\mathrm{n} / \mathrm{a}$ & $\mathrm{n} / \mathrm{a}$ \\
\hline ICP.W.Pb & $\mu \mathrm{g} / \mathrm{g}$ & $8.15 \mathrm{E}+01$ & $\mathrm{n} / \mathrm{a}$ & $\mathrm{n} / \mathrm{a}$ & $\mathrm{n} / \mathrm{a}$ & $\mathrm{n} / \mathrm{a}$ \\
\hline ICP.a.Li ${ }^{1}$ & $\mu \mathrm{g} / \mathrm{g}$ & $8.14 \mathrm{E}+00$ & $\mathrm{n} / \mathrm{a}$ & $\mathrm{n} / \mathrm{a}$ & $\mathrm{n} / \mathrm{a}$ & $\mathrm{n} / \mathrm{a}$ \\
\hline ICP.f. $\mathrm{Li}^{1}$ & $\mu \mathrm{g} / \mathrm{g}$ & $1.92 \mathrm{E}+02$ & $\mathrm{n} / \mathrm{a}$ & $\mathrm{n} / \mathrm{a}$ & $\mathrm{n} / \mathrm{a}$ & $\mathrm{n} / \mathrm{a}$ \\
\hline ICP.w.Li ${ }^{1}$ & $\mu \mathrm{g} / \mathrm{g}$ & $8.15 E+00$ & $\mathrm{n} / \mathrm{a}$ & $\mathrm{n} / \mathrm{a}$ & $\mathrm{n} / \mathrm{a}$ & $n / a$ \\
\hline ICP.a. $\mathbf{M g}^{1}$ & $\mu \mathrm{g} / \mathrm{g}$ & $8.14 \mathrm{E}+01$ & $\mathrm{n} / \mathrm{a}$ & $\mathrm{n} / \mathrm{a}$ & $\mathrm{n} / \mathrm{a}$ & $\mathrm{n} / \mathrm{a}$ \\
\hline ICP.w.Mg ${ }^{1}$ & $\mu \mathrm{g} / \mathrm{g}$ & $8.15 E+01$ & $\mathrm{n} / \mathrm{a}$ & $\mathrm{n} / \mathrm{a}$ & $\mathrm{n} / \mathrm{a}$ & $\mathrm{n} / \mathrm{a}$ \\
\hline ICP.a.Mn & $\mu \mathrm{g} / \mathrm{g}$ & $6.46 \mathrm{E}+01$ & $9.80 \mathrm{E}+00$ & 2 & $2.24 \mathrm{E}+01$ & $1.07 \mathrm{E}+02$ \\
\hline ICP.f.Mn ${ }^{1}$ & $\mu \mathrm{g} / \mathrm{g}$ & $1.92 \mathrm{E}+02$ & $\mathrm{n} / \mathrm{a}$ & $\mathrm{n} / \mathrm{a}$ & $\mathrm{n} / \mathrm{a}$ & $\mathrm{n} / \mathrm{a}$ \\
\hline ICP.w. $\mathrm{Mn}^{1}$ & $\mu \mathrm{g} / \mathrm{g}$ & $8.15 E+00$ & $\mathrm{n} / \mathrm{a}$ & $\mathrm{n} / \mathrm{a}$ & $\mathrm{n} / \mathrm{a}$ & $\mathrm{n} / \mathrm{a}$ \\
\hline ICP.a. $\mathrm{Mo}^{2}$ & $\mu \mathrm{g} / \mathrm{g}$ & $5.53 \mathrm{E}+01$ & $5.54 \mathrm{E}+00$ & 2 & $3.14 \mathrm{E}+01$ & $7.91 \mathrm{E}+01$ \\
\hline ICP.w.Mo & $\mu \mathrm{g} / \mathrm{g}$ & $5.77 \mathrm{E}+01$ & $7.80 \mathrm{E}+00$ & 2 & $2.42 \mathrm{E}+01$ & $9.13 \mathrm{E}+01$ \\
\hline ICP.a. $\mathrm{Nd}^{\mathrm{I}}$ & $\mu \mathrm{g} / \mathrm{g}$ & $8.14 \mathrm{E}+01$ & $\mathrm{n} / \mathrm{a}$ & $\mathrm{n} / \mathrm{a}$ & $\mathrm{n} / \mathrm{a}$ & $\mathrm{n} / \mathrm{a}$ \\
\hline ICP.w.Nd ${ }^{1}$ & $\mu \mathrm{g} / \mathrm{g}$ & $8.15 \mathrm{E}+01$ & $\mathrm{n} / \mathrm{a}$ & $\mathrm{n} / \mathrm{a}$ & $\mathrm{n} / \mathrm{a}$ & $n / a$ \\
\hline
\end{tabular}


Table B3-6. 95 Percent Two-Sided Confidence Interval for the Mean Concentration for Composite Sample Data. (4 sheets)

\begin{tabular}{|c|c|c|c|c|c|c|}
\hline Haillofo & Writs & \% & 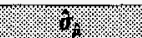 & 610 & Wh & 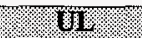 \\
\hline ICP.a.Ni & $\mu \mathrm{g} / \mathrm{g}$ & $5.11 \mathrm{E}+01$ & $1.60 \mathrm{E}+00$ & 2 & $4.42 \mathrm{E}+01$ & $5.79 \mathrm{E}+01$ \\
\hline ICP.f. $\mathrm{Ni}^{1}$ & $\mu \mathrm{g} / \mathrm{g}$ & $9.51 \mathrm{E}+02$ & $\mathrm{n} / \mathrm{a}$ & $\mathrm{n} / \mathrm{a}$ & $\mathrm{n} / \mathrm{a}$ & $\bar{n} / \mathrm{a}$ \\
\hline ICP.w. $\mathrm{Ni}^{1}$ & $\mu \mathrm{g} / \mathrm{g}$ & $1.76 \mathrm{E}+01$ & $\mathrm{n} / \mathrm{a}$ & $\mathrm{n} / \mathrm{a}$ & $\mathrm{n} / \mathrm{a}$ & $\mathrm{n} / \mathrm{a}$ \\
\hline Nitrate & $\mu \mathrm{g} / \mathrm{g}$ & $2.56 \mathrm{E}+05$ & $5.55 \mathrm{E}+04$ & 2 & $1.7 \overline{5 E}+04$ & $4.95 \mathrm{E}+05$ \\
\hline Nitrite & $\mu \mathrm{g} / \mathrm{g}$ & $6.37 \bar{E}+04$ & $9.49 \mathrm{E}+03$ & 2 & $2.2 \overline{\mathrm{E}}+04$ & $1.05 \mathrm{E}+05$ \\
\hline Oxalate & $\mu \mathrm{g} / \mathrm{g}$ & $4.64 E+03$ & $1.69 \mathrm{E}+02$ & 2 & $3.91 \mathrm{E}+03$ & $5.36 \mathrm{E}+03$ \\
\hline Phosphate & $\mu \mathrm{g} / \mathrm{g}$ & $1.24 \mathrm{E}+04$ & $1.76 \mathrm{E}+03$ & 2 & $4.8 \overline{3 E}+03$ & $2 . \overline{00 \mathrm{E}+04}$ \\
\hline ICP.a.P & $\mu \mathrm{g} / \mathrm{g}$ & $3.61 \overline{E+03}$ & $3.85 \mathrm{E}+02$ & 2 & $1.96 \mathrm{E}+03$ & $5.27 \overline{\mathrm{E}+03}$ \\
\hline ICP.f.P & $\mu \mathrm{g} / \mathrm{g}$ & $3.85 \mathrm{E}+03$ & $\mathrm{n} / \mathrm{a}$ & $\mathrm{n} / \mathrm{a}$ & $\bar{n} / \mathrm{a}$ & $\mathrm{n} / \mathrm{a}$ \\
\hline ICP.w.P & $\mu \mathrm{g} / \mathrm{g}$ & $4.00 \mathrm{E}+03$ & $4 . \overline{71 E+02}$ & 2 & $1.97 \mathrm{E}+03$ & $6.02 \mathrm{E}+03$ \\
\hline ICP.a.K & $\mu \mathrm{g} / \mathrm{g}$ & $1.58 \mathrm{E}+03$ & $1.86 \mathrm{E}+02$ & 2 & $7.79 \mathrm{E}+02$ & $2.38 \mathrm{E}+03$ \\
\hline ICP.w.K & $\mu \mathrm{g} / \mathrm{g}$ & $1.67 \mathrm{E}+03$ & $1.97 \bar{E}+02$ & 2 & $8.22 \mathrm{E}+02$ & $2.52 \mathrm{E}+03$ \\
\hline ICP.a.Sm ${ }^{1}$ & $\mu \mathrm{g} / \mathrm{g}$ & $8.14 \mathrm{E}+01$ & $\mathrm{n} / \mathrm{a}$ & $\mathrm{n} / \mathrm{a}$ & $\mathrm{n} / \mathrm{a}$ & $\mathrm{n} / \mathrm{a}$ \\
\hline ICP.w.Sm ${ }^{1}$ & $\mu \mathrm{g} / \mathrm{g}$ & $8.15 \mathrm{E}+01$ & $\mathrm{n} / \mathrm{a}$ & $\mathrm{n} / \mathrm{a}$ & $\mathrm{n} / \mathrm{a}$ & $\mathrm{n} / \mathrm{a}$ \\
\hline ICP.a.Se ${ }^{1}$ & $\mu \mathrm{g} / \mathrm{g}$ & $8.14 \mathrm{E}+01$ & $\mathrm{n} / \mathrm{a}$ & $\mathrm{n} / \mathrm{a}$ & $\mathrm{n} / \mathrm{a}$ & $\mathrm{n} / \mathrm{a}$ \\
\hline ICP.w.Se ${ }^{1}$ & $\mu \mathrm{g} / \mathrm{g}$ & $8.15 \mathrm{E}+01$ & $\mathrm{n} / \mathrm{a}$ & $\mathrm{n} / \mathrm{a}$ & $\mathrm{n} / \mathrm{a}$ & $\mathrm{n} / \mathrm{a}$ \\
\hline ICP.a.Si & $\mu \mathrm{g} / \mathrm{g}$ & $1.19 \mathrm{E}+02$ & $1.75 \mathrm{E}+01$ & 2 & $4.36 \mathrm{E}+01$ & $1.94 \mathrm{E}+02$ \\
\hline ICP.f.Si ${ }^{1}$ & $\mu \mathrm{g} / \mathrm{g}$ & $9.59 \mathrm{E}+02$ & $\mathrm{n} / \mathrm{a}$ & $n / a$ & $\mathrm{n} / \mathrm{a}$ & $\mathrm{n} / \mathrm{a}$ \\
\hline ICP.w.Si & $\mu \mathrm{g} / \mathrm{g}$ & $2.83 \mathrm{E}+02$ & $2.69 \mathrm{E}+01$ & 2 & $1.67 \mathrm{E}+02$ & $3.98 \mathrm{E}+02$ \\
\hline ICP.a. $\mathrm{Ag}^{\mathrm{I}}$ & $\mu \mathrm{g} / \mathrm{g}$ & $1.19 \mathrm{E}+01$ & $\mathrm{n} / \mathrm{a}$ & $\mathrm{n} / \mathrm{a}$ & $\mathrm{n} / \mathrm{a}$ & $\mathrm{n} / \mathrm{a}$ \\
\hline ICP.w. $\mathrm{Ag}^{1}$ & $\mu \mathrm{g} / \mathrm{g}$ & $1.14 \mathrm{E}+01$ & $\mathrm{n} / \mathrm{a}$ & $\mathrm{n} / \mathrm{a}$ & $\mathrm{n} / \mathrm{a}$ & $\mathrm{n} / \mathrm{a}$ \\
\hline ICP.a.Na & $\mu \mathrm{g} / \mathrm{g}$ & $2.06 \mathrm{E}+05$ & $7.08 \mathrm{E}+03$ & 2 & $1.75 \mathrm{E}+05$ & $2.36 \mathrm{E}+05$ \\
\hline ICP.f.Na & $\mu \mathrm{g} / \mathrm{g}$ & $2.82 \mathrm{E}+05$ & $1.97 \mathrm{E}+04$ & 2 & $1.97 \mathrm{E}+05$ & $3.66 \mathrm{E}+05$ \\
\hline ICP.w.Na & $\mu \mathrm{g} / \mathrm{g}$ & $2.16 \mathrm{E}+05$ & $7.06 \mathrm{E}+03$ & 2 & $1.86 \mathrm{E}+05$ & $2.46 \mathrm{E}+05$ \\
\hline ICP.a.Sr ${ }^{1}$ & $\mu \mathrm{g} / \mathrm{g}$ & $8.14 \mathrm{E}+00$ & $\mathrm{n} / \mathrm{a}$ & $\mathrm{n} / \mathrm{a}$ & $\mathrm{n} / \mathrm{a}$ & $\mathrm{n} / \mathrm{a}$ \\
\hline ICP.w.Sr ${ }^{1}$ & $\mu \mathrm{g} / \mathrm{g}$ & $8.15 \mathrm{E}+00$ & $n / a$ & $\mathrm{n} / \mathrm{a}$ & $\mathrm{n} / \mathrm{a}$ & $\mathrm{n} / \mathrm{a}$ \\
\hline $\mathrm{Sr}-89 / 90$ & $\mu \mathrm{Ci} / \mathrm{g}$ & $9 . \overline{32 \mathrm{E}+00}$ & $1.27 \mathrm{E}+00$ & 2 & $3.85 E+00$ & $1.48 \mathrm{E}+01$ \\
\hline Sulfate & $\mu \mathrm{g} / \mathrm{g}$ & $1.76 \bar{E}+04$ & $3.23 \mathrm{E}+02$ & 2 & $1.62 \mathrm{E}+04$ & $1.90 \mathrm{E}+04$ \\
\hline ICP.a.S & $\mu \mathrm{g} / \mathrm{g}$ & $5.05 \mathrm{E}+03$ & $3.64 \mathrm{E}+02$ & 2 & $3.49 \mathrm{E}+03$ & $6.62 \mathrm{E}+03$ \\
\hline ICP.w.S & $\mu \mathrm{g} / \mathrm{g}$ & $5.53 \mathrm{E}+03$ & $1.89 \mathrm{E}+02$ & 2 & $4.71 \mathrm{E}+03$ & $6.34 \mathrm{E}+03$ \\
\hline ICP.a.Tl $^{1}$ & $\mu \mathrm{g} / \mathrm{g}$ & $1.63 \mathrm{E}+02$ & $\mathrm{n} / \mathrm{a}$ & $\mathrm{n} / \mathrm{a}$ & $\mathrm{n} / \mathrm{a}$ & $\mathrm{n} / \mathrm{a}$ \\
\hline ICP.w.Tl $^{1}$ & $\mu \mathrm{g} / \mathrm{g}$ & $1.63 \mathrm{E}+02$ & $n / a$ & $\mathrm{n} / \mathrm{a}$ & $\mathrm{n} / \mathrm{a}$ & $\mathrm{n} / \mathrm{a}$ \\
\hline
\end{tabular}


Table B3-6. 95 Percent Two-Sided Confidence Interval for the Mean Concentration for Composite Sample Data. (4 sheets)

\begin{tabular}{|c|c|c|c|c|c|c|}
\hline snaryte & Whits & (4) & 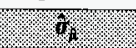 & oll & $13 \%$ & UI \\
\hline ICP.a.Ti & $\mu \mathrm{g} / \mathrm{g}$ & $8.14 \mathrm{E}+00$ & $\mathrm{n} / \mathrm{a}$ & $\mathrm{n} / \mathrm{a}$ & $\mathrm{n} / \mathrm{a}$ & $\mathrm{n} / \mathrm{a}$ \\
\hline ICP.w.Ti ${ }^{1}$ & $\mu \mathrm{g} / \mathrm{g}$ & $8.15 \mathrm{E}+00$ & $\mathrm{n} / \mathrm{a}$ & $\mathrm{n} / \mathrm{a}$ & $\mathrm{n} / \mathrm{a}$ & $\mathrm{n} / \mathrm{a}$ \\
\hline TIC & $\mu \mathrm{g} / \mathrm{g}$ & $8.43 E+03$ & $7.86 \mathrm{E}+02$ & 2 & $5.05 \mathrm{E}+03$ & $1.18 \mathrm{E}+04$ \\
\hline TOC & $\mu \mathrm{g} / \mathrm{g}$ & $4.47 \mathrm{E}+03$ & $4.16 \mathrm{E}+02$ & 2 & $2.68 \mathrm{E}+03$ & $6.26 \mathrm{E}+03$ \\
\hline ICP.a.U ${ }^{1}$ & $\mu \mathrm{g} / \mathrm{g}$ & $4.07 \mathrm{E}+02$ & $\mathrm{n} / \mathrm{a}$ & $\mathrm{n} / \mathrm{a}$ & $\mathrm{n} / \mathrm{a}$ & $\mathrm{n} / \mathrm{a}$ \\
\hline ICP.f.U $U^{1}$ & $\mu \mathrm{g} / \mathrm{g}$ & $9.59 \mathrm{E}+03$ & $\mathrm{n} / \mathrm{a}$ & $\mathrm{n} / \mathrm{a}$ & $\mathrm{n} / \mathrm{a}$ & $\mathrm{n} / \mathrm{a}$ \\
\hline ICP.w. $U^{1}$ & $\mu \mathrm{g} / \mathrm{g}$ & $4.07 \mathrm{E}+02$ & $\mathrm{n} / \mathrm{a}$ & $\mathrm{n} / \mathrm{a}$ & $n / a$ & $n / a$ \\
\hline Uranium & $\mu \mathrm{g} / \mathrm{g}$ & $1.87 \mathrm{E}+02$ & $3.07 \mathrm{E}+01$ & 2 & $5.50 \overline{\mathrm{E}+01}$ & $3.20 \mathrm{E}+02$ \\
\hline ICP.a. V ${ }^{1}$ & $\mu \mathrm{g} / \mathrm{g}$ & $4.07 \mathrm{E}+01$ & $\mathrm{n} / \mathrm{a}$ & $\mathrm{n} / \mathrm{a}$ & $\mathrm{n} / \mathrm{a}$ & $\mathrm{n} / \mathrm{a}$ \\
\hline ICP.w. $V^{1}$ & $\mu \mathrm{g} / \mathrm{g}$ & $4.07 \mathrm{E}+01$ & $\mathrm{n} / \mathrm{a}$ & $\mathrm{n} / \mathrm{a}$ & $\mathrm{n} / \mathrm{a}$ & $\mathrm{n} / \mathrm{a}$ \\
\hline ICP.a.Zn & $\mu \mathrm{g} / \mathrm{g}$ & $4.56 \mathrm{E}+01$ & $1.76 \mathrm{E}+01$ & 2 & $0.00 \mathrm{E}+00$ & $1.21 \mathrm{E}+02$ \\
\hline ICP.w.Zn & $\mu \mathrm{g} / \mathrm{g}$ & $2.34 \mathrm{E}+01$ & $6.58 \mathrm{E}+00$ & 2 & $0.00 \mathrm{E}+00$ & $5.18 \mathrm{E}+01$ \\
\hline ICP.a. $\mathrm{Zr}^{1}$ & $\mu \mathrm{g} / \mathrm{g}$ & $8.14 \mathrm{E}+00$ & $\mathrm{n} / \mathrm{a}$ & $\mathrm{n} / \mathrm{a}$ & $\mathrm{n} / \mathrm{a}$ & $\mathrm{n} / \mathrm{a}$ \\
\hline ICP.w. $\mathrm{Zr}^{1}$ & $\mu \mathrm{g} / \mathrm{g}$ & $8.15 E+00$ & $\mathrm{n} / \mathrm{a}$ & $\mathrm{n} / \mathrm{a}$ & $\mathrm{n} / \mathrm{a}$ & $\mathrm{n} / \mathrm{a}$ \\
\hline$\%$ Water & $\%$ & $3.15 \mathrm{E}+01$ & $2.14 \mathrm{E}+00$ & 2 & $2.23 \mathrm{E}+01$ & $4.07 \mathrm{E}+01$ \\
\hline DSC & Joules/g & $3.12 \mathrm{E}+01$ & $3.12 \mathrm{E}+01$ & 2 & $0.00 \mathrm{E}+00$ & $1.65 \mathrm{E}+02$ \\
\hline
\end{tabular}

Notes:

${ }^{1}$ More than 50 percent of the analytical results were less-than values; therefore, confidence intervals were not computed.

${ }^{2}$ Fewer than 50 percent, but some of the results, were less-than values.

In addition to core composite data, segment level data from tank 241-U-108 was also available. The supernatant sample data and solid sample data were analyzed separately. Supernatant samples were present only in segment 1 of both cores. Mean concentration estimates, along with 95 percent confidence intervals on the mean, are given in Table B3-6 for the solid segment sample data and Table B3-7 for the supernatant segment sample data.

Table B3-7. 95 Percent Two-Sided Confidence Interval for the Mean Concentration for Solid Segment Sample Data. (3 sheets)

\begin{tabular}{|c|c|c|c|c|c|c|}
\hline aninge & U1to & t. & x. & al & 18 & 4 \\
\hline ICP.f.Al & $\mu \mathrm{g} / \mathrm{g}$ & $1.65 \mathrm{E}+04$ & $2.74 \mathrm{E}+03$ & 2 & $4.70 \mathrm{E}+03$ & $2.83 \mathrm{E}+04$ \\
\hline Am- $241^{1}$ & $\mu \mathrm{Ci} / \mathrm{g}$ & $6.61 \mathrm{E}-01$ & $\mathrm{n} / \mathrm{a}$ & $\mathrm{n} / \mathrm{a}$ & $\mathrm{n} / \mathrm{a}$ & $\mathrm{n} / \mathrm{a}$ \\
\hline ICP.f.Bi ${ }^{1}$ & $\mu \mathrm{g} / \mathrm{g}$ & $2.03 \mathrm{E}+03$ & $\mathrm{n} / \mathrm{a}$ & $\mathrm{n} / \mathrm{a}$ & $\mathrm{n} / \mathrm{a}$ & $\mathrm{n} / \mathrm{a}$ \\
\hline
\end{tabular}


Table B3-7. 95 Percent Two-Sided Confidence Interval for the Mean Concentration for Solid Segment Sample Data. (3 sheets)

\begin{tabular}{|c|c|c|c|c|c|c|}
\hline linilyte. & Unis: & $1:$ & 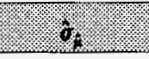 & $\mathrm{dr}$ & I.1. & ur. \\
\hline Bromide $^{1}$ & $\mu \mathrm{g} / \mathrm{g}$ & $1.04 \mathrm{E}+03$ & $\mathrm{n} / \mathrm{a}$ & $\mathrm{n} / \mathrm{a}$ & $\mathrm{n} / \mathrm{a}$ & $\mathrm{n} / \mathrm{a}$ \\
\hline Bulk density & --- & $1.74 \mathrm{E}+00$ & $4.06 \mathrm{E}-02$ & 2 & $1.56 \mathrm{E}+00$ & $1.91 \mathrm{E}+00$ \\
\hline ICP.f. $\mathrm{Ca}^{1}$ & $\mu \mathrm{g} / \mathrm{g}$ & $2.03 \mathrm{E}+03$ & $\mathrm{n} / \mathrm{a}$ & $\mathrm{n} / \mathbf{a}$ & $\mathrm{n} / \mathbf{a}$ & $\mathrm{n} / \mathrm{a}$ \\
\hline Cs-137 & $\mu \mathrm{Ci} / \mathrm{g}$ & $1.41 \mathrm{E}+02$ & $6.48 \mathrm{E}+00$ & 2 & $1.13 \mathrm{E}+02$ & $1.69 \mathrm{E}+02$ \\
\hline Chloride & $\mu \mathrm{g} / \mathrm{g}$ & $3.45 \mathrm{E}+03$ & $2.23 \mathrm{E}+02$ & 2 & $2.49 \mathrm{E}+03$ & $4.41 \mathrm{E}+03$ \\
\hline ICP.f.Cr & $\mu \mathrm{g} / \mathrm{g}$ & 4. $12 \mathrm{E}+03$ & $2.88 \mathrm{E}+02$ & 2 & $2.88 \mathrm{E}+03$ & $5.36 \mathrm{E}+03$ \\
\hline Co-60' & $\mu \mathrm{Ci} / \mathrm{g}$ & $2.10 \mathrm{E}-02$ & $\mathrm{n} / \mathrm{a}$ & $\mathrm{n} / \mathrm{a}$ & $\mathrm{n} / \mathrm{a}$ & $\mathrm{n} / \mathrm{a}$ \\
\hline $\mathrm{Eu}-154^{1}$ & $\mu \mathrm{Ci} / \mathrm{g}$ & $8.23 \mathrm{E}-02$ & $\mathrm{n} / \mathrm{a}$ & $\mathrm{n} / \mathrm{a}$ & $\mathrm{n} / \mathrm{a}$ & $\mathrm{n} / \mathrm{a}$ \\
\hline Eu-155 ${ }^{1}$ & $\mu \mathrm{Ci} / \mathrm{g}$ & $3.03 \mathrm{E}-01$ & $\mathrm{n} / \mathrm{a}$ & $\mathrm{n} / \mathrm{a}$ & $\mathrm{n} / \mathrm{a}$ & $\mathrm{n} / \mathrm{a}$ \\
\hline Fluoride $^{2}$ & $\mu \mathrm{g} / \mathrm{g}$ & $9.56 \mathrm{E}+02$ & $1.86 \mathrm{E}+02$ & 2 & $1.55 \mathrm{E}+02$ & $1.76 \mathrm{E}+03$ \\
\hline Alpha $^{2}$ & $\mu \mathrm{Ci} / \mathrm{g}$ & $5.25 \mathrm{E}-02$ & $1.01 \mathrm{E}-02$ & 2 & $9.11 \mathrm{E}-03$ & $9.59 \mathrm{E}-02$ \\
\hline ICP.f.Fe ${ }^{1}$ & $\mu \mathrm{g} / \mathrm{g}$ & $1.03 \mathrm{E}+03$ & $\mathrm{n} / \mathrm{a}$ & $\mathrm{n} / \mathrm{a}$ & $\mathrm{n} / \mathrm{a}$ & $\mathrm{n} / \mathrm{a}$ \\
\hline ICP.f. $\mathrm{Li}^{1}$ & $\mu \mathrm{g} / \mathrm{g}$ & $2.03 E+02$ & $\mathrm{n} / \mathrm{a}$ & $\mathrm{n} / \mathrm{a}$ & $\mathrm{n} / \mathrm{a}$ & $\mathrm{n} / \mathbf{a}$ \\
\hline ICP.f.Mn ${ }^{1}$ & $\mu \mathrm{g} / \mathrm{g}$ & $2.30 \mathrm{E}+02$ & $n / a$ & $\mathrm{n} / \mathrm{a}$ & $\mathrm{n} / \mathrm{a}$ & $\mathrm{n} / \mathrm{a}$ \\
\hline ICP.f. $\mathrm{Ni}^{1}$ & $\mu \mathrm{g} / \mathrm{g}$ & $4.06 \mathrm{E}+02$ & $\mathrm{n} / \mathrm{a}$ & $\mathrm{n} / \mathrm{a}$ & $\mathrm{n} / \mathrm{a}$ & n/a \\
\hline Nitrate & $\mu \mathrm{g} / \mathrm{g}$ & $2.95 \mathrm{E}+05$ & $2.45 \mathrm{E}+04$ & 2 & $1.90 \mathrm{E}+05$ & $4.01 \mathrm{E}+05$ \\
\hline Nitrite & $\mu \mathrm{g} / \mathrm{g}$ & $5.00 \mathrm{E}+04$ & $2.14 \mathrm{E}+03$ & 2 & $4.08 \mathrm{E}+04$ & $5.92 \mathrm{E}+04$ \\
\hline Oxalate $^{2}$ & $\mu \mathrm{g} / \mathrm{g}$ & $4.35 \mathrm{E}+03$ & $5.15 \mathrm{E}+02$ & 2 & $2.13 \mathrm{E}+03$ & $6.56 \mathrm{E}+03$ \\
\hline Phosphate & $\mu \mathrm{g} / \mathrm{g}$ & $1.72 \mathrm{E}+04$ & $4.85 E+03$ & 2 & $0.00 \mathrm{E}+00$ & $3.81 \mathrm{E}+04$ \\
\hline ICP.f.P ${ }^{1}$ & $\mu \mathrm{g} / \mathrm{g}$ & $7.04 \mathrm{E}+03$ & $\mathrm{n} / \mathrm{a}$ & $\mathrm{n} / \mathrm{a}$ & $\mathrm{n} / \mathrm{a}$ & $\mathrm{n} / \mathrm{a}$ \\
\hline ICP.f.Si ${ }^{1}$ & $\mu \mathrm{g} / \mathrm{g}$ & $1.08 \mathrm{E}+03$ & $\mathrm{n} / \mathrm{a}$ & $\mathrm{n} / \mathrm{a}$ & $\mathrm{n} / \mathrm{a}$ & $\mathrm{n} / \mathrm{a}$ \\
\hline ICP.f. Na & $\mu \mathrm{g} / \mathrm{g}$ & $2.41 \mathrm{E}+05$ & $5.31 E+03$ & 2 & $2.18 \mathrm{E}+05$ & $2.64 \mathrm{E}+05$ \\
\hline Sr-89/90 & $\mu \mathrm{Ci} / \mathrm{g}$ & $1.01 \mathrm{E}+01$ & $1.99 \mathrm{E}+00$ & 2 & $1.50 \mathrm{E}+00$ & $1.86 \mathrm{E}+01$ \\
\hline Sulfate ${ }^{2}$ & $\mu \mathrm{g} / \mathrm{g}$ & $1.51 \mathrm{E}+04$ & $1.01 \mathrm{E}+03$ & 2 & $1.08 \mathrm{E}+04$ & $1.95 \mathrm{E}+04$ \\
\hline TIC & $\mu \mathrm{g} / \mathrm{g}$ & $7.97 \mathrm{E}+03$ & $6.03 E+02$ & 2 & $5.37 \mathrm{E}+03$ & $1.06 \mathrm{E}+04$ \\
\hline TOC & $\mu \mathrm{g} / \mathrm{g}$ & $4.16 \mathrm{E}+03$ & $2.50 \mathrm{E}+02$ & 2 & $3.09 \mathrm{E}+03$ & $5.24 \mathrm{E}+03$ \\
\hline ICP.f. $U^{1}$ & $\mu \mathrm{g} / \mathrm{g}$ & $1.01 \mathrm{E}+04$ & $\mathrm{n} / \mathbf{a}$ & $\mathrm{n} / \mathrm{a}$ & $\mathrm{n} / \mathrm{a}$ & $\mathrm{n} / \mathrm{a}$ \\
\hline
\end{tabular}


Table B3-7. 95 Percent Two-Sided Confidence Interval for the Mean Concentration for Solid Segment Sample Data. (3 sheets)

\begin{tabular}{|c|c|c|c|c|c|c|}
\hline Analye & Uiliss & it. & (3. & df & 121. & (11) \\
\hline$\%$ Water & $\%$ & $3.43 \mathrm{E}+01$ & $1.57 \mathrm{E}+00$ & 2 & $2.75 \mathrm{E}+01$ & $4.10 \mathrm{E}+01$ \\
\hline DSC & Joules $/ \mathrm{g}$ & $4.23 \mathrm{E}+01$ & $8.63 \mathrm{E}+00$ & 2 & $5.18 \mathrm{E}+00$ & $7.95 \mathrm{E}+01$ \\
\hline
\end{tabular}

Notes:

'More than 50 percent of the analytical results were less than values; therefore, confidence intervals were not computed.

${ }^{2}$ Fewer than 50 percent, but some of the results, were less-than values.

\section{B3.4.2 Analysis of Variance Models}

A statistical model is needed to account for the spatial and measurement variability in $\hat{\sigma}_{\hat{\mu}}$. This cannot be done using an ordinary standard deviation of the data (Snedecor and Cochran 1980).

The statistical model fit to the composite sample data and supernatant segment sample data is

$$
\begin{aligned}
& Y_{i j}=\mu+C_{i}+A_{i j}, \\
& i=1, \ldots, a, j=1, \ldots, n_{i},
\end{aligned}
$$

where

$$
\begin{array}{ll}
\mathrm{Y}_{\mathrm{ij}} & =\text { laboratory results from the } \mathrm{j}^{\text {th }} \text { duplicate from the } \mathrm{i}^{\text {ih }} \text { core in the tank } \\
\mu & =\text { the grand mean } \\
\mathrm{C}_{\mathrm{i}} & =\text { the effect of the } \mathrm{i}^{\mathrm{ith}} \text { core } \\
\mathrm{A}_{\mathrm{ij}} & =\text { the effect of the } \mathrm{j}^{\mathrm{ith}} \text { analytical result from the } \mathrm{i}^{\text {th }} \text { core } \\
\mathrm{a} & =\text { the number of cores } \\
\mathrm{n}_{\mathrm{i}} & =\text { the number of analytical results from the } \mathrm{i}^{\mathrm{ith}} \text { location. }
\end{array}
$$


The variable $C_{i}$ is assumed to be a random effect. This variable and $A_{i j}$ are assumed to be uncorrelated and normally distributed with means zero and variances $\sigma^{2}(\mathrm{C})$ and $\sigma^{2}(\mathrm{~A})$, respectively. Estimates of $\sigma^{2}(\mathrm{C})$ and $\sigma^{2}(\mathrm{~A})$ were obtained using REML techniques. This method, applied to variance component estimation, is described in Harville (1977). The statistical results were obtained using the statistical analysis package S-PLUS (Statistical Sciences 1993).

The statistical model fit to the solid segment sample data is

$$
\begin{gathered}
\mathrm{Y}_{\mathrm{ijkmn}}=\mu+\mathrm{C}_{\mathrm{i}}+\mathrm{S}_{\mathrm{ij}}+\mathrm{L}_{\mathrm{ijk}}+\mathrm{A}_{\mathrm{ijkm},}, \\
\mathrm{i}=1, \ldots, \mathrm{a}, \mathrm{j}=1, \ldots, \mathrm{b}_{\mathrm{i}}, \mathrm{k}=1, \ldots, \mathrm{c}_{\mathrm{ij}}, \mathrm{m}=1, \ldots, \mathrm{n}_{\mathrm{ijk}},
\end{gathered}
$$

where

$$
\begin{aligned}
& \mathrm{Y}_{\mathrm{ijkm}}=\text { laboratory results from the } \mathrm{m}^{\text {th }} \text { duplicate from the } \mathrm{k}^{\mathrm{th}} \text { location in the } \\
& \mathrm{j}^{\text {th }} \text { segment in the } \mathrm{i}^{\text {th }} \text { core in the tank, } \\
& \mu \quad=\quad \text { the grand mean } \\
& \mathrm{C}_{\mathrm{i}}=\text { the effect of the } \mathrm{i}^{\text {th }} \text { core } \\
& S_{\mathrm{ij}}=\text { the effect of the } \mathrm{j}^{\mathrm{ith}} \text { segment from the } \mathrm{i}^{\text {th }} \text { core } \\
& \mathrm{L}_{\mathrm{ijk}}=\text { the effect of the } \mathrm{k}^{\text {th }} \text { location from the } \mathrm{j}^{\text {th }} \text { segment in the } \mathrm{i}^{\mathrm{th}} \text { core } \\
& \mathrm{A}_{\mathrm{ijkm}}=\text { the effect of the } \mathrm{m}^{\text {th }} \text { analytical result from the } \mathrm{k}^{\text {th }} \text { location in the } \mathrm{j}^{\mathrm{th}} \\
& \text { segment in the } i^{\text {th }} \text { core } \\
& \text { a }=\text { the number of cores } \\
& b_{i}=\text { the number of segments in the } i^{\text {th }} \text { core } \\
& \mathrm{c}_{\mathrm{ij}}=\text { the number of locations from the } \mathrm{j}^{\mathrm{j}} \text { segment in the } \mathrm{i}^{\text {th }} \text { core } \\
& \mathrm{n}_{\mathrm{ijk}}=\text { the number of analytical results from the } \mathrm{k}^{\text {th }} \text { location in } \mathrm{j}^{\mathrm{it}} \text { segment in }
\end{aligned}
$$

The variable $C_{i}, S_{i j}$, and, $L_{i j k}$ are assumed to be random effects. These variables and $A_{i j k m}$ are assumed to be uncorrelated and normally distributed with means zero and variances $\sigma^{2}(\mathrm{C}), \sigma^{2}(\mathrm{~S}), \sigma^{2}(\mathrm{~L})$, and $\sigma^{2}(\mathrm{~A})$, respectively. Estimates of $\sigma^{2}(\mathrm{C}), \sigma^{2}(\mathrm{~S}), \sigma^{2}(\mathrm{~L})$, and $\sigma^{2}(\mathrm{~A})$ were obtained using REML techniques. This method, applied to variance component estimation, 
is described in Reference 2. The statistical results were obtained using statistical analysis package S-PLUS (Statistical Sciences 1993).

\section{B3.4.3 Inventory}

After the sample means are calculated for the tank for each analyte, the sampling based inventory may be calculated. Because the analyte concentrations above are presented in terms of a mass basis concentration, the total mass of waste in the tank is needed to estimate inventories. The total mass of waste is derived from the tank volume (from surveillance) and the tank solids density. The tank volume for solids is $1,680 \mathrm{~kL}$ and the tank volume for liquids is $91 \mathrm{~kL}$ (Hanlon 1996). The densities used for these estimates are $1.74 \mathrm{~g} / \mathrm{mL}$ for segment sample data, $1.71 \mathrm{~g} / \mathrm{mL}$ for composite sample data, and $1.40 \mathrm{~g} / \mathrm{mL}$ for liquid sample data (Bell 1996a). The inventory (and 95 percent confidence interval on the inventory) of each of the analytes is presented in Table B3-8 for composite sample data, Table B3-9 for solid segment sample data, and Table B3-10 for liquid segment sample data. No analytical-based inventories were calculated for analytes where more than 50 percent of the results were below the detection limit.

Table B3-8. 95 Percent Two-Sided Confidence Interval for the Mean Concentration for Supernatant Segment Sample Data. (2 sheets)

\begin{tabular}{|c|c|c|c|c|c|c|}
\hline Whing & Winto & X. & 6 & of & Y. & S16. \\
\hline ICP.d.Al & $\mu \mathrm{g} / \mathrm{mL}$ & $3.29 \mathrm{E}+04$ & $2.44 \mathrm{E}+03$ & 3 & $2.51 \mathrm{E}+04$ & $4.07 \mathrm{E}+04$ \\
\hline$\overline{\mathrm{ICP}}$.d.Sb ${ }^{\mathrm{T}}$ & $\mu \mathrm{g} / \mathrm{mL}$ & $2.41 \mathrm{E}+01$ & $\mathrm{n} / \mathrm{a}$ & $\mathrm{n} / \mathrm{a}$ & $\mathrm{n} / \mathrm{a}$ & $n / a$ \\
\hline ICP.d.As ${ }^{1}$ & $\mu \mathrm{g} / \mathrm{mL}$ & $4.01 \mathrm{E}+01$ & $\mathrm{n} / \mathrm{a}$ & $\mathrm{n} / \mathrm{a}$ & $\mathrm{n} / \mathrm{a}$ & $\mathrm{n} / \mathrm{a}$ \\
\hline ICP.d.Ba ${ }^{1}$ & $\mu \mathrm{g} / \mathrm{mL}$ & $2.01 \mathrm{E}+01$ & $\mathrm{n} / \mathrm{a}$ & $\mathrm{n} / \mathrm{a}$ & $\mathrm{n} / \mathrm{a}$ & $\mathrm{n} / \mathrm{a}$ \\
\hline ICP.d.Be ${ }^{1}$ & $\mu \mathrm{g} / \mathrm{mL}$ & $2.00 \mathrm{E}+00$ & $\mathrm{n} / \mathrm{a}$ & $\mathrm{n} / \mathrm{a}$ & $n / a$ & $\mathrm{n} / \mathrm{a}$ \\
\hline ICP.d.Bi ${ }^{1}$ & $\mu \mathrm{g} / \mathrm{mL}$ & $4.01 \mathrm{E}+01$ & $\mathrm{n} / \mathrm{a}$ & $\mathrm{n} / \mathrm{a}$ & $\mathrm{n} / \mathrm{a}$ & $\mathrm{n} / \mathrm{a}$ \\
\hline ICP.d.B & $\mu \mathrm{g} / \mathrm{mL}$ & $9.12 \mathrm{E}+01$ & $2.87 \mathrm{E}+00$ & 2 & $7.89 \bar{E}+01$ & $1.04 \mathrm{E}+02$ \\
\hline Bromide $^{1}$ & $\mu \mathrm{g} / \mathrm{mL}$ & $5.64 \mathrm{E}+02$ & $\mathrm{n} / \mathrm{a}$ & $\mathrm{n} / \mathrm{a}$ & $\mathrm{n} / \mathrm{a}$ & $\mathrm{n} / \mathrm{a}$ \\
\hline ICP.d.Cd ${ }^{1}$ & $\mu \mathrm{g} / \mathrm{mL}$ & $2.00 \mathrm{E}+00$ & $\mathrm{n} / \mathrm{a}$ & $\mathrm{n} / \mathrm{a}$ & $n / a$ & $\mathrm{n} / \mathrm{a}$ \\
\hline $\mathrm{ICP}^{-d . \mathrm{Ca}^{1}}$ & $\mu \mathrm{g} / \mathrm{mL}$ & $4.06 \mathrm{E}+01$ & $\mathrm{n} / \mathrm{a}$ & $\mathrm{n} / \mathrm{a}$ & $\mathrm{n} / \mathrm{a}$ & $\mathrm{n} / \mathrm{a}$ \\
\hline ICP.d.Ce ${ }^{1}$ & $\mu \mathrm{g} / \mathrm{mL}$ & $4.01 \mathrm{E}+01$ & $\mathrm{n} / \mathrm{a}$ & $\mathrm{n} / \mathrm{a}$ & $\mathrm{n} / \mathrm{a}$ & $\mathrm{n} / \mathrm{a}$ \\
\hline Chloride & $\mu \mathrm{g} / \mathrm{mL}$ & $8.94 \mathrm{E}+03$ & $2.43 E+02$ & 3 & $8.17 \mathrm{E}+03$ & $9 . \overline{72 E+03}$ \\
\hline ICP.d.Cr & $\mu \mathrm{g} / \mathrm{mL}$ & $1.28 \mathrm{E}+03$ & $9.70 \mathrm{E}+01$ & 2 & $8.59 E+02$ & $1.69 E+03$ \\
\hline ICP.d.Co ${ }^{1}$ & $\mu \mathrm{g} / \mathrm{mL}$ & $8.02 \mathrm{E}+00$ & $\mathrm{n} / \mathrm{a}$ & $\mathrm{n} / \mathrm{a}$ & $\mathrm{n} / \mathrm{a}$ & $\mathrm{n} / \mathbf{a}$ \\
\hline ICP.d.Cu ${ }^{1}$ & $\mu \mathrm{g} / \mathrm{mL}$ & $4.01 E+00$ & $\mathrm{n} / \mathrm{a}$ & $\mathrm{n} / \mathrm{a}$ & $\mathrm{n} / \mathrm{a}$ & $\mathrm{n} / \mathrm{a}$ \\
\hline Fluoride $^{2}$ & $\mu \mathrm{g} / \mathrm{mL}$ & $7.90 \mathrm{E}+02$ & $2.69 \mathrm{E}+02$ & 3 & $0.00 \mathrm{E}+00$ & $1.36 \mathrm{E}+03$ \\
\hline Alpha $^{\top}$ & $\mu \mathrm{Ci} / \mathrm{mL}$ & $9.79 \mathrm{E}-03$ & $\mathrm{n} / \mathrm{a}$ & $\mathrm{n} / \mathrm{a}$ & $\mathrm{n} / \mathrm{a}$ & $n / a$ \\
\hline ICP.d.Fe & $\mu \mathrm{g} / \mathrm{mL}$ & $3.33 \mathrm{E}+01$ & $2.65 \mathrm{E}+00$ & 3 & $2.49 \mathrm{E}+01$ & $4.18 \mathrm{E}+01$ \\
\hline ICP.d.La & $\mu \mathrm{g} / \mathrm{mL}$ & $2.01 \mathrm{E}+01$ & $\mathrm{n} / \mathrm{a}$ & $\mathrm{n} / \mathrm{a}$ & $\mathrm{n} / \mathrm{a}$ & $\mathrm{n} / \mathrm{a}$ \\
\hline ICP.d.Pb ${ }^{1}$ & $\mu \mathrm{g} / \mathrm{mL}$ & $4.01 \mathrm{E}+01$ & $\mathrm{n} / \mathrm{a}$ & $\mathrm{n} / \mathrm{a}$ & $\mathrm{n} / \mathrm{a}$ & $\mathrm{n} / \mathrm{a}$ \\
\hline
\end{tabular}


Table B3-8. 95 Percent Two-Sided Confidence Interval for the Mean Concentration for Supernatant Segment Sample Data. (2 sheets)

\begin{tabular}{|c|c|c|c|c|c|c|}
\hline 4ardo & Writs & $\%$ & 6 & dif & 16. & W1. \\
\hline ICP.d.Li ${ }^{1}$ & $\mu \mathrm{g} / \mathrm{mL}$ & $4.02 \mathrm{E}+00$ & $\mathrm{n} / \mathrm{a}$ & $\mathrm{n} / \mathrm{a}$ & $n / a$ & $\mathrm{n} / \mathrm{a}$ \\
\hline ICP.d.Mg ${ }^{1}$ & $\mu \mathrm{g} / \mathrm{mL}$ & $4.01 \mathrm{E}+01$ & $\mathrm{n} / \mathrm{a}$ & $n / \mathbf{a}$ & $\mathrm{n} / \mathrm{a}$ & $\mathrm{n} / \mathrm{a}$ \\
\hline ICP.d. $\mathrm{Mn}^{1}$ & $\mu \mathrm{g} / \mathrm{mL}$ & $4.01 E+00$ & $\mathrm{n} / \mathrm{a}$ & $\mathrm{n} / \mathrm{a}$ & $\mathrm{n} / \mathrm{a}$ & $n / a$ \\
\hline ICP.d.Mo & $\mu \mathrm{g} / \mathrm{mL}$ & $1 . \overline{23 E+02}$ & $1.48 \mathrm{E}+00$ & 2 & $1.16 \mathrm{E}+02$ & $1.29 \mathrm{E}+02$ \\
\hline ICP.d.Nd ${ }^{1}$ & $\mu \mathrm{g} / \mathrm{mL}$ & $4.01 \mathrm{E}+01$ & $n / a$ & $\mathrm{n} / \mathrm{a}$ & $\mathrm{n} / \mathrm{a}$ & $\mathrm{n} / \mathrm{a}$ \\
\hline ICP.d.Ni & $\mu \mathrm{g} / \mathrm{mL}$ & $3.13 \mathrm{E}+01$ & $1.62 \mathrm{E}+00$ & 2 & $2 . \overline{43 E}+01$ & $3.82 \mathrm{E}+01$ \\
\hline Nitrate & $\mu \mathrm{g} / \mathrm{mL}$ & $1.81 \mathrm{E}+05$ & $5.06 \mathrm{E}+03$ & 3 & $1.65 \mathrm{E}+\overline{05}$ & $1.97 \mathrm{E}+05$ \\
\hline Nitrite & $\mu \mathrm{g} / \mathrm{mL}$ & $1.30 \mathrm{E}+05$ & $4.83 \mathrm{E}+03$ & 3 & $1.14 \mathrm{E}+05$ & $1.45 \mathrm{E}+05$ \\
\hline Oxalate $^{1}$ & $\mu \mathrm{g} / \mathrm{mL}$ & $5.20 \mathrm{E}+02$ & $\mathrm{n} / \mathrm{a}$ & $n / a$ & $\mathrm{n} / \mathrm{a}$ & $\mathrm{n} / \mathrm{a}$ \\
\hline Phosphate & $\mu \mathrm{g} / \mathrm{mL}$ & $2.50 \mathrm{E}+03$ & $2.45 \mathrm{E}+02$ & 3 & $1 . \overline{2 E+03}$ & $3.28 \mathrm{E}+03$ \\
\hline ICP.d.P & $\mu \mathrm{g} / \mathrm{mL}$ & $1.02 \mathrm{E}+03$ & $2.72 \mathrm{E}+01$ & 2 & $8.99 \mathrm{E}+02$ & $1.13 \mathrm{E}+03$ \\
\hline $\mathrm{Pu}-239 / 240^{1}$ & $\mu \mathrm{Ci} / \mathrm{mL}$ & $8.09 \mathrm{E}-05$ & $\mathrm{n} / \mathrm{a}$ & $\mathrm{n} / \mathrm{a}$ & $n / a$ & $\mathrm{n} / \mathrm{a}$ \\
\hline ICP.d.K & $\mu \mathrm{g} / \mathrm{mL}$ & $4.01 \mathrm{E}+03$ & $6.66 \mathrm{E}+01$ & 2 & $3.72 \mathrm{E}+03$ & $4.30 \mathrm{E}+03$ \\
\hline ICP.d.Sm ${ }^{3}$ & $\mu \mathrm{g} / \mathrm{mL}$ & $4.01 \mathrm{E}+01$ & $\mathrm{n} / \mathrm{a}$ & $\mathrm{n} / \mathrm{a}$ & $\mathrm{n} / \mathrm{a}$ & $\mathrm{n} / \mathrm{a}$ \\
\hline ICP.d.Se ${ }^{1}$ & $\mu \mathrm{g} / \mathrm{mL}$ & $4.01 \mathrm{E}+01$ & $\mathrm{n} / \mathrm{a}$ & $\mathrm{n} / \mathrm{a}$ & $\mathrm{n} / \mathrm{a}$ & $n / a$ \\
\hline ICP.d.Si & $\mu \mathrm{g} / \mathrm{mL}$ & $1.95 \mathrm{E}+02$ & $5.09 \bar{E}+00$ & 2 & $1.73 \mathrm{E}+02$ & $2.17 \mathrm{E}+02$ \\
\hline ICP.d.Ag & $\mu \mathrm{g} / \mathrm{mL}$ & $1.63 \mathrm{E}+01$ & $3.82 \mathrm{E}-01$ & 2 & $1.47 \mathrm{E}+01$ & $1.79 \mathrm{E}+01$ \\
\hline ICP.d.Na & $\mu \mathrm{g} / \mathrm{mL}$ & $2.43 \mathrm{E}+05$ & $1.52 \mathrm{E}+\overline{04}$ & 3 & $1.94 \mathrm{E}+05$ & $2.91 \mathrm{E}+05$ \\
\hline SpG & none & $1.40 \mathrm{E}+00$ & $1.24 \mathrm{E}-02$ & 3 & $1.36 \mathrm{E}+00$ & $1.4 \overline{4 E+00}$ \\
\hline ICP.d.Sr ${ }^{1}$ & $\mu \mathrm{g} / \mathrm{mL}$ & $4.01 \mathrm{E}+00$ & $\mathrm{n} / \mathrm{a}$ & $\mathrm{n} / \mathrm{a}$ & $\mathrm{n} / \mathrm{a}$ & $\mathrm{n} / \mathrm{a}$ \\
\hline Sulfate & $\mu \mathrm{g} / \mathrm{mL}$ & $3.63 E+03$ & $4.00 \mathrm{E}+02$ & 3 & $2.36 \mathrm{E}+03$ & $4.90 \mathrm{E}+03$ \\
\hline ICP.d.S & $\mu \mathrm{g} / \mathrm{mL}$ & $1.41 \mathrm{E}+03$ & $8.94 \mathrm{E}+00$ & 2 & $1.37 \mathrm{E}+03$ & $1.45 \mathrm{E}+03$ \\
\hline ICP.d.TI $^{1}$ & $\mu \mathrm{g} / \mathrm{mL}$ & $8.02 \mathrm{E}+01$ & $\mathrm{n} / \mathrm{a}$ & $\mathrm{n} / \mathrm{a}$ & $\mathrm{n} / \mathrm{a}$ & $\mathrm{n} / \mathrm{a}$ \\
\hline ICP.d.Ti $^{1}$ & $\mu \mathrm{g} / \mathrm{mL}$ & $4.01 \mathrm{E}+00$ & $\mathrm{n} / \mathrm{a}$ & $\mathrm{n} / \mathrm{a}$ & $\mathrm{n} / \mathrm{a}$ & $\mathrm{n} / \mathrm{a}$ \\
\hline TIC & $\mu \mathrm{g} / \mathrm{mL}$ & $4.40 \mathrm{E}+03$ & $9.79 \mathrm{E}+01$ & 2 & $3.98 \mathrm{E}+03$ & $4.82 \mathrm{E}+03$ \\
\hline TOC & $\mu \mathrm{g} / \mathrm{mL}$ & $7.16 \mathrm{E}+03$ & $9.46 \mathrm{E}+01$ & 3 & $6.86 \mathrm{E}+03$ & $7.46 \mathrm{E}+03$ \\
\hline ICP.d.U ${ }^{1}$ & $\mu \mathrm{g} / \mathrm{mL}$ & $2.00 \mathrm{E}+02$ & $\mathrm{n} / \mathrm{a}$ & $\mathrm{n} / \mathrm{a}$ & $\mathrm{n} / \mathrm{a}$ & $\mathrm{n} / \mathrm{a}$ \\
\hline ICP.d. V & $\mu \mathrm{g} / \mathrm{mL}$ & $2.01 \mathrm{E}+01$ & $\mathrm{n} / \mathrm{a}$ & $\mathrm{n} / \mathrm{a}$ & $\mathrm{n} / \mathrm{a}$ & $\mathrm{n} / \mathrm{a}$ \\
\hline ICP.d.Zn & $\mu \mathrm{g} / \mathrm{mL}$ & $4.52 \mathrm{E}+01$ & $7.20 \mathrm{E}+00$ & 2 & $1.42 \mathrm{E}+01$ & $7.62 \mathrm{E}+01$ \\
\hline ICP.d. $\mathrm{Zr}^{1}$ & $\mu \mathrm{g} / \mathrm{mL}$ & $4.01 \mathrm{E}+00$ & $\mathrm{n} / \mathrm{a}$ & $\mathrm{n} / \mathrm{a}$ & $\mathrm{n} / \mathrm{a}$ & $\mathrm{n} / \mathrm{a}$ \\
\hline$\%$ Water & $\%$ & $5.02 \mathrm{E}+01$ & $4.57 \mathrm{E}-01$ & 3 & $4.86 \mathrm{E}+01$ & $5.15 E+01$ \\
\hline DSC & Joules/g & $5.69 \mathrm{E}+01$ & $1.94 \mathrm{E}+01$ & 3 & $0.00 \mathrm{E}+00$ & $1.19 E+02$ \\
\hline
\end{tabular}

Notes:

${ }^{1}$ More than 50 percent of the analytical results were less than values; therefore, confidence intervals were not computed.

${ }^{2}$ Fewer than 50 percent, but some of the results, were less-than values. 
Table B3-9. Analytical-Based Inventory for Composite Sample Data for Tank 241-U-108. (2 sheets)

\begin{tabular}{|c|c|c|c|}
\hline . & 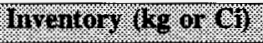 & (2): & (1) \\
\hline ICP.a.Al & $5.29 \mathrm{E}+04$ & $6.34 \mathrm{E}+03$ & $9.95 \mathrm{E}+04$ \\
\hline ICP.f.Al & $5.41 \mathrm{E}+04$ & $1.4 \overline{8 \mathrm{E}+04}$ & $9.33 \mathrm{E}+04$ \\
\hline ICP.w.Al & $4.74 \mathrm{E}+04$ & $1.65 \mathrm{E}+04$ & $7.83 \mathrm{E}+04$ \\
\hline ICP.a.B & $2.31 \mathrm{E}+02$ & $3.77 \bar{E}+01$ & $4 . \overline{24} \mathrm{E}+\overline{02}$ \\
\hline ICP.w.B & $1.51 \mathrm{E}+03$ & $1.15 \mathrm{E}+03$ & $1 . \overline{86 \mathrm{E}+\overline{03}}$ \\
\hline ICP.a.Cd & $1.28 \mathrm{E}+01$ & $6.91 \mathrm{E}+00$ & $1.87 \mathrm{E}+01$ \\
\hline ICP.a.Ca & $4.47 \mathrm{E}+02$ & $2.22 \mathrm{E}+02$ & $6.72 \mathrm{E}+02$ \\
\hline ICP.w.Ca & $3.31 \mathrm{E}+02$ & $1.36 \mathrm{E}+02$ & $\overline{5.2} 6 \overline{\mathrm{E}+02}$ \\
\hline Cs -137 & $4.66 \mathrm{E}+05$ & $2.95 \mathrm{E}+05$ & $6.38 \bar{E}+05$ \\
\hline Chloride & $1.26 \mathrm{E}+04$ & $4.27 \mathrm{E}+03$ & $2.09 \mathrm{E}+04$ \\
\hline ICP.a.Cr & $1.10 \overline{\mathrm{E}}+04$ & $9.35 \mathrm{E}+03$ & $1.26 \mathrm{E}+04$ \\
\hline ICP.f.Cr & $1.17 \overline{\mathrm{E}+04}$ & $8.51 \mathrm{E}+03$ & $1.48 \mathrm{E}+04$ \\
\hline ICP.w.Cr & $1.02 \mathrm{E}+03$ & $3.14 \mathrm{E}+02$ & $1.73 \mathrm{E}+03$ \\
\hline Alpha & $1.26 \mathrm{E}+02$ & $5.33 E+01$ & $1.99 \mathrm{E}+02$ \\
\hline Beta & $4.79 \mathrm{E}+05$ & $3.48 \mathrm{E}+05$ & $6.10 \mathrm{E}+05$ \\
\hline ICP.a.Fe & $5.04 \mathrm{E}+02$ & $3.07 \mathrm{E}+02$ & $7 . \overline{00 \mathrm{E}+02}$ \\
\hline ICP.a.Mn & $1.85 \mathrm{E}+02$ & $6.43 \mathrm{E}+01$ & $3.07 \mathrm{E}+02$ \\
\hline ICP.a.Mo & $1.59 \mathrm{E}+02$ & $9.02 \mathrm{E}+01$ & $2.27 \mathrm{E}+02$ \\
\hline ICP.w.Mo & $1.66 \mathrm{E}+02$ & $6.94 \mathrm{E}+01$ & $2.62 \mathrm{E}+02$ \\
\hline ICP.a.Ni & $1.47 \mathrm{E}+02$ & $1.27 \mathrm{E}+02$ & $1.66 \mathrm{E}+02$ \\
\hline Nitrate & $7.36 \mathrm{E}+05$ & $5.02 \mathrm{E}+04$ & $1.42 \mathrm{E}+0 \overline{6}$ \\
\hline Nitrite & $1.83 \mathrm{E}+05$ & $6.58 \mathrm{E}+04$ & $3.00 \mathrm{E}+05$ \\
\hline Oxalate & $1.33 \mathrm{E}+04$ & $1.12 \mathrm{E}+04$ & $1.5 \overline{4 \mathrm{E}+04}$ \\
\hline Phosphate & $3.56 \mathrm{E}+04$ & $1.39 \mathrm{E}+04$ & $5.74 \mathrm{E}+04$ \\
\hline ICP.a.P & $1.04 \mathrm{E}+04$ & $5.62 \mathrm{E}+03$ & $1.51 \mathrm{E}+04$ \\
\hline ICP.w.P & $1.15 \mathrm{E}+04$ & $5.65 \mathrm{E}+03$ & $1.73 \mathrm{E}+04$ \\
\hline ICP.a.K & $4.54 \overline{\mathrm{E}+03}$ & $2.24 \mathrm{E}+03$ & $6.84 \mathrm{E}+03$ \\
\hline ICP.w.K & $4.80 \mathrm{E}+03$ & $2.36 \mathrm{E}+03$ & $7.23 \mathrm{E}+03$ \\
\hline ICP.a.Si & $3.42 \mathrm{E}+02$ & $1.25 \mathrm{E}+02$ & $5.58 \mathrm{E}+02$ \\
\hline ICP.w.Si & $8.12 \mathrm{E}+02$ & $4.80 \mathrm{E}+02$ & $1.14 \mathrm{E}+03$ \\
\hline ICP.a. Na & $5.91 \mathrm{E}+05$ & $5.03 \mathrm{E}+05$ & $6.79 \mathrm{E}+05$ \\
\hline ICP.f.Na & $8.09 \mathrm{E}+05$ & $5.65 \mathrm{E}+05$ & $1.05 \mathrm{E}+06$ \\
\hline ICP.w.Na & $6.20 \mathrm{E}+05$ & $5.33 \mathrm{E}+05$ & $7.07 \mathrm{E}+05$ \\
\hline Sr-89/90 & $2.68 \mathrm{E}+04$ & $1.11 \mathrm{E}+04$ & $4.25 \mathrm{E}+04$ \\
\hline
\end{tabular}


Table B3-9. Analytical-Based Inventory for Composite Sample Data for Tank 241-U-108. (2 sheets)

\begin{tabular}{|c|c|c|c|}
\hline Nmilo & Hinontory $\left(k_{p}, 01<1\right.$ & W. & W. \\
\hline Sulfate & $5.05 \mathrm{E}+04$ & $4.65 \mathrm{E}+04$ & $5.45 E+04$ \\
\hline ICP.a.S & $1.45 \mathrm{E}+04$ & $1.00 \mathrm{E}+04$ & $1 . \overline{90 \mathrm{E}+04}$ \\
\hline ICP.w.S & $1.59 \mathrm{E}+04$ & $1.35 \mathrm{E}+04$ & $1.82 \mathrm{E}+04$ \\
\hline TIC & $2.42 \mathrm{E}+04$ & $1.45 \mathrm{E}+04$ & $3.39 \mathrm{E}+04$ \\
\hline TOC & $1.28 \mathrm{E}+04$ & $7.70 \mathrm{E}+03$ & $1.80 \mathrm{E}+04$ \\
\hline Uranium & $5.38 \mathrm{E}+02$ & $1.58 \mathrm{E}+02$ & $9 . \overline{18 E}+02$ \\
\hline ICP.a. $\mathrm{Zn}$ & $1.31 \mathrm{E}+02$ & $0.00 \mathrm{E}+00$ & $3.49 \mathrm{E}+02$ \\
\hline ICP.w.Zn & $6.73 \mathrm{E}+01$ & $0.00 \mathrm{E}+00$ & $1.49 \mathrm{E}+02$ \\
\hline$\%$ Water & $9.05 \mathrm{E}+05$ & $6.41 E+05$ & $1.17 \mathrm{E}+06$ \\
\hline
\end{tabular}


Table B3-10. Analytical-Based Inventory for Solid Segment Sample Data for Tank 241-U-108.

\begin{tabular}{|c|c|c|c|}
\hline 6rapyfo & Trventory (kg or (Y) & $4.1 .1 \%$ & (1) \\
\hline$\%$ Water & $1.00 \mathrm{E}+06$ & $8.04 \mathrm{E}+05$ & $1.20 \mathrm{E}+06$ \\
\hline Cs-137 & $4.11 \mathrm{E}+05$ & $3.30 \mathrm{E}+05$ & $4.93 \mathrm{E}+05$ \\
\hline Alpha & $1.54 \mathrm{E}+02$ & $2.66 \mathrm{E}+01$ & $2.80 \overline{\mathrm{E}}+02$ \\
\hline Sr- $89 / 90$ & $2.94 \mathrm{E}+04$ & $4.38 \mathrm{E}+03$ & $5.45 \mathrm{E}+04$ \\
\hline ICP.f.Al & $4.8 \overline{2 \mathrm{E}+04}$ & $1.37 \mathrm{E}+04$ & $8.26 \mathrm{E}+04$ \\
\hline Chloride & $1.01 \mathrm{E}+04$ & $7.28 \mathrm{E}+03$ & $1.29 \mathrm{E}+04$ \\
\hline ICP.f.Cr & $1.20 \mathrm{E}+\overline{04}$ & $8.42 \mathrm{E}+03$ & $1.57 \overline{\mathrm{E}+04}$ \\
\hline Fluoride & $2.79 \mathrm{E}+03$ & $4.54 \mathrm{E}+02$ & $5.13 \bar{E}+03$ \\
\hline Nitrate & $8.63 \mathrm{E}+05$ & $5.54 \mathrm{E}+05$ & $1.17 \mathrm{E}+06$ \\
\hline Nitrite & $1.46 \mathrm{E}+05$ & $1.19 \mathrm{E}+05$ & $1.73 E+05$ \\
\hline Oxalate & $1.27 \mathrm{E}+04$ & $6.23 \mathrm{E}+03$ & $1.92 \mathrm{E}+04$ \\
\hline Phosphate & $5.03 \mathrm{E}+04$ & 0.00 & $1.11 \mathrm{E}+05$ \\
\hline ICP.f.Na & $7.04 \mathrm{E}+05$ & $6.37 \mathrm{E}+05$ & $7.71 \mathrm{E}+05$ \\
\hline Sulfate & $4.41 \mathrm{E}+04$ & $3.14 \mathrm{E}+04$ & $5.69 \mathrm{E}+04$ \\
\hline TIC & $2.33 \mathrm{E}+04$ & $1.57 \overline{\mathrm{E}+04}$ & $3.09 \mathrm{E}+04$ \\
\hline TOC & $1.22 \mathrm{E}+04$ & $9.03 \mathrm{E}+03$ & $1.53 \mathrm{E}+04$ \\
\hline
\end{tabular}


Table B3-11. Analytical-Based Inventory for Liquid Segment Sample Data for Tank 241-U-108.

\begin{tabular}{|c|c|c|c|}
\hline Amalyte & Invertom lig of (i) & 11 & 11. \\
\hline Water & $1.46 \mathrm{E}+06$ & $1.42 \mathrm{E}+06$ & $1.50 \mathrm{E}+06$ \\
\hline ICP.d.AI & $2.99 \mathrm{E}+03$ & $2.29 \mathrm{E}+03$ & $3.70 \mathrm{E}+03$ \\
\hline ICP.d.B & $8.30 \mathrm{E}+00$ & $7.18 \mathrm{E}+\overline{00}$ & $9.43 \overline{\mathrm{E}}+00$ \\
\hline Chloride & $8.14 \mathrm{E}+02$ & $7.43 \mathrm{E}+02$ & $8.84 \mathrm{E}+02$ \\
\hline ICP.d.Cr & $1.16 \mathrm{E}+02$ & $7.82 \mathrm{E}+01$ & $1.54 \mathrm{E}+02$ \\
\hline Fluoride & $4.59 \mathrm{E}+01$ & $0.00 \mathrm{E}+00$ & $1.24 \mathrm{E}+02$ \\
\hline ICP.d.Fe & $3.03 \mathrm{E}+00$ & $2.26 \mathrm{E}+00$ & $3.80 \overline{\mathrm{E}}+00$ \\
\hline ICP.d.Mo & $1.12 \mathrm{E}+01$ & $1.06 \mathrm{E}+01$ & $1.17 \mathrm{E}+01$ \\
\hline ICP.d.Ni & $2.84 \mathrm{E}+00$ & $2.21 \mathrm{E}+00$ & $3.48 \mathrm{E}+00$ \\
\hline Nitrate & $1.65 \mathrm{E}+04$ & $1.50 \mathrm{E}+04$ & $1.80 \mathrm{E}+04$ \\
\hline Nitrite & $1.18 \mathrm{E}+04$ & $1.04 \mathrm{E}+04$ & $1.32 \mathrm{E}+04$ \\
\hline Phosphate & $2.27 \mathrm{E}+02$ & $1.56 \mathrm{E}+02$ & $2.98 \mathrm{E}+02$ \\
\hline ICP.d.P & $9.25 \mathrm{E}+01$ & $8.18 \mathrm{E}+01$ & $1.03 \mathrm{E}+02$ \\
\hline ICP.d.K & $3.65 \mathrm{E}+02$ & $3.39 \mathrm{E}+02$ & $3.91 \mathrm{E}+02$ \\
\hline ICP.d.Si & $1.77 \overline{\mathrm{E}+01}$ & $1 . \overline{57 \mathrm{E}+01}$ & $1.97 \mathrm{E}+01$ \\
\hline ICP.d.Ag & $1.48 \mathrm{E}+00$ & $1.33 \mathrm{E}+00$ & $1.63 \mathrm{E}+00$ \\
\hline ICP.d.Na & $2.21 \mathrm{E}+04$ & $1.77 \mathrm{E}+04$ & $2.65 \mathrm{E}+04$ \\
\hline Sulfate & $3.30 \mathrm{E}+02$ & $2.14 \mathrm{E}+02$ & $4.46 \mathrm{E}+02$ \\
\hline ICP.d.S & $1.28 \mathrm{E}+02$ & $1.25 \mathrm{E}+02$ & $1.32 \overline{E+02}$ \\
\hline TIC & $4.00 \mathrm{E}+02$ & $3.62 \mathrm{E}+02$ & $4.38 \mathrm{E}+02$ \\
\hline$\overline{\text { TOC }}$ & $6.52 \mathrm{E}+02$ & $6.24 \mathrm{E}+02$ & $6.79 \mathrm{E}+02$ \\
\hline ICP.d.Zn & $4.11 \mathrm{E}+00$ & $1.29 \mathrm{E}+00$ & $6.93 \mathrm{E}+00$ \\
\hline
\end{tabular}




\section{B4.0 APPENDIX B REFERENCES}

Agnew, S. F., J. Boyer, R. A. Corbin, T. B. Duran, J. R. Fitzpatrick, K. A. Jurgensen, T. P. Ortiz, and B. L. Young, 1996a, Hanford Tank Chemical and Radionuclide Inventories: HDW Model Rev. 3, LA-UR-96-858, Los Alamos National Laboratory, Los Alamos, New Mexico.

Agnew, S. F., R. A. Corbin, T. B. Duran, K. A. Jurgensen, T. P. Ortiz, and B. L. Young, 1996b, Waste Status and Transaction Record Summary for the Southwest Quadrant of the Hanford 200 East Area, WHC-SD-WM-TI-614, Rev. 1, Westinghouse Hanford Company, Richland, Washington.

Baldwin, D. L., R. W. Stromatt, and W. I. Winters, 1994, Comparative Study of Total Organic Carbon Methods for High-Level Mixed Waste, PNL-SA-23718, Pacific Northwest National Laboratory, Richland, Washington.

Bell, K. E., 1996a, Final Report for Tank 241-U-108, Push-Mode Core Samples 141, 145, and 146 Analytical Results for the Final Report, WHC-SD-WM-DP-198, Rev. 0, Westinghouse Hanford Company, Richland, Washington.

Bell, K. E., 1996b, Safety Screening/Immediate Notification for Tank 241-U-108, (internal memorandum 79400-96-161 to J. N. Appel and Distribution, August 8), Westinghouse Hanford Company, Richland, Washington.

Brown, T. M., S. J. Eberlein, J. W. Hunt, and T. J. Kunthara, 1995, Tank Waste Characterization Basis, WHC-SD-WM-TA-164, Rev. 1, Westinghouse Hanford Company, Richland, Washington.

Carothers, K. E., 1994, Data Quality Objectives for the Waste Compatibility Program, WHC-SD-WM-DQO-001, Rev. 0, Westinghouse Hanford Company, Richland, Washington.

Cash, R. J., 1996, Scope Increase of 'Data Quality Objective to Support Resolution of the Organic Complexant Safety Issue,' Rev. 2, (internal memorandum 79300-96-029 to S. J. Eberlein, July 12), Westinghouse Hanford Company, Richland, Washington.

DeLorenzo, D. S., J. H. Rutherford, D. J. Smith, D. B. Hiller, K. W. Johnson, and B. C. Simpson, 1994, Tank Characterization Reference Guide, WHC-SD-WM-TI-648, Rev. 0, Westinghouse Hanford Company, Richland, Washington.

Dukelow, G. T., J. W. Hunt, H. Babad, and J. E Meacham, 1995, Tank Safety Screening Data Quality Objective, WHC-SD-WM-SP-004, Rev. 2, Westinghouse Hanford Company, Richland, Washington. 
Esch, R. A., 1995, Waste Compatibility Results for 241-U-108 Grab Samples, (internal memorandum 75970-95-043, Rev. 1 to M. J. Sutey, R. D. Schreiber, S. D. Estey, and N. W. Kirch, July 24), Westinghouse Hanford Company, Richland, Washington.

Hanlon, B. M., 1996, Waste Tank Summary Report for the Month Ending September 30, 1996, WHC-EP-0182-102, Westinghouse Hanford Company, Richland, Washington.

Harville, D. A., 1977, "Maximum Likelihood Approaches to Variance Component Estimation and to Related Problems," Journal of the American Statistical Association, [vol. no. unknown], pp. 320-340.

Hewitt, E. R., 1996, Tank Waste Remediation System Resolution of Potentially Hazardous Vapor Issues, WHC-SD-TWR-RPT-001, Rev. 0, Westinghouse Hanford Company, Richland, Washington.

Homi, C. S., 1995, Vapor Sampling and Analysis Plan, WHC-SD-WM-TP-335, Rev. OF, Westinghouse Hanford Company, Richland, Washington.

Homi, C. S., 1996, Tank 241-U-108 Push Mode Core Sampling and Analysis Plan, WHC-SD-WM-TSAP-049, Rev. 0, Westinghouse Hanford Company, Richland, Washington.

Horton, J. E., 1975, Analyses of Sludge Sample From Tank 108-U, (letter [number unknown] to W. R. Christensen [employer unknown], September 29), Atlantic Richfield Hanford Company, Richland, Washington.

Izmerov, N. F., 1982, "Toxicometric Parameters of Industrial Toxic Chemicals Under Single Exposure", Moscow, Centre of International Projects, GKNT, vol. 30, [pp. unknown].

Kristofzski, J. G., 1996, Directions for 'Opportunistic Analyses,' (internal memorandum 74900-96-168 to J. H. Baldwin and Distribution, September 11), Westinghouse Hanford Company, Richland, Washington.

Mahlum, D. D., J. Y. Young, and R. E. Weller, 1994, Toxicologic Evaluation of Analytes From Tank 241-C-108, PNL-10189, Pacific Northwest National Laboratories, Richland, Washington.

Mahon, R. D., 1995, Vapor and Gas Sampling of Single-Shell Tank 241-U-108 Using the Vapor Sampling System, WHC-SD-WM-RPT-180, Rev. 1, Westinghouse Hanford Company, Richland, Washington.

Meacham, J. E., 1995, Test Plan for Samples From Hanford Waste Tanks 241-BY-103, $B Y-104, B Y-105, B Y-106, B Y-108, B Y-110, T Y-103, U-105, U-107, U-108$, and $U-109$, WHC-SD-WM-TP-378, Rev. 0, Westinghouse Hanford Company, Richland, Washington. 
NIOSH 1994, NIOSH Pocket Guide to Chemical Hazards, 017-033-00473-1, U. S. Government Printing Office, Washington, DC 20402.

Osborne, J. W., J. L. Huckaby, B. A. Pulsipher, E. R. Hewitt, D. D. Mahlum, J. Y. Young, and C. M. Anderson, 1995, Data Quality Objectives for Generic In-Tank Health and Safety Issue Resolution, WHC-SD-WM-DQO-002, Rev. 1, Westinghouse Hanford Company, Richland, Washington.

Osborne, J. W., and J. L. Huckaby, 1994, Program Plan for the Resolution of Tank Vapor Issues, WHC-EP-0562, Rev. 1, Westinghouse Hanford Company, Richland, Washington.

Puryear, D. A., 1971, Characterization of S, $U$ and SX Waste Tanks, (letter to J. O. Skolrud [employer unknown], September 21), Atlantic Richfield Hanford Company, Richland, Washington.

Rice, A. D., 1996, Correction of Spike Recovery for Persulfate TIC/TOC Analyses on Tank Samples, (letter 9655794, November 20), Rust Federal Services of Hanford Inc., Richland, Washington

Sant, W.H., 1974, 242-S Feed Samples, Number T-151, Sample Point 108-U, (letter to R. L. Walser [employer unknown], January 21, Atlantic Richfield Hanford Company, Richland, Washington.

Schreiber, R. D., 1995, Compatibility Grab Sampling and Analysis Plan, WHC-SD-WM-TP-330, Rev. 0, Westinghouse Hanford Company, Richland, Washington.

Simpson, B. C., and D. J. McCain, 1995, Historical Model Evaluation Data Requirements, WHC-SD-WM-DQO-018, Rev. 0A, Westinghouse Hanford Company, Richland, Washington.

Snedecor, G. W., and W. G. Cochran, 1980, Statistical Methods, 7th Edition, Iowa State University Press, Ames, Iowa.

Statistical Sciences, Inc., 1993, S-PLUS Reference Manual, Version 3.2, StatSci, a division of MathSoft, Inc., Seattle, Washington.

Thomas, B. L., T. W. Clauss, J. C. Evana, B. D. McVeety, K. H. Pool, K. B. Olsen, J. S. Fruchter, and M. W. Ligotke, 1996, Headspace Vapor Characterization of Hanford Waste Tank 241-U-108: Results from Samples Collected on 08/29/95, PNNL-10961, Pacific Northwest National Laboratory, Richland, Washington. 
Turner, D. A., H. Babad, L. L. Buckley, and J. E. Meacham, 1996, Data Quality Objective to Support Resolution of the Organic Complexant Safety Issue, WHC-SD-WM-DQO-006, Rev. 2, Westinghouse Hanford Company, Richland, Washington.

WHC 1996, Tank U-108 Core Sampling Work Package WS-95-00250, Westinghouse Hanford Company, Richland, Washington.

Wilkins, N. E., R. E. Bauer, and D. M. Ogden, 1997, Results of Vapor Space Monitoring of Flammable Gas Watch List Tank, HNF-SD-WM-TI-797, Rev. 1, Lockheed Martin Hanford Corporation, Richland, Washington.

Winkelman, W. D., 1996, Tank 24I-U-108 Tank Characterization Plan, WHC-SD-WM-TP-315, Rev. 3, Westinghouse Hanford Company, Richland, Washington. 
HNF-SD-WM-ER-639 Rev. 0

APPENDIX C
STATISTICAL ANALYSIS FOR ISSUE RESOLUTION

C-1 
HNF-SD-WM-ER-639 Rev. 0

This page left blank intentionally. 


\section{APPENDIX C}

\section{STATISTICAL ANALYSIS FOR ISSUE RESOLUTION}

\section{C1.0 STATISTICS FOR SAFETY SCREENING AND ORGANIC DQOS}

The safety screening (Dukelow et al. 1995) and organic (Turner et al. 1996) DQOs define acceptable decision confidence limits in terms of one-sided 95 percent confidence intervals. In this appendix, one-sided confidence limits supporting the safety screening and organic DQOs are calculated for tank 241-U-108. All data in this section are from the final laboratory data package for the 1996 core sampling event for tank 241-U-108 (Bell 1996).

Confidence intervals were computed for each sample number from tank 241-U-108 analytical data. The sample numbers and confidence intervals are provided in Table C1-1 for alpha and Table C1-2 for DSC.

The upper limit (UL) of a one-sided 95 percent confidence interval on the mean is

$$
\hat{\mu}+\mathrm{t}_{(\mathrm{df}, 0.05)} * \hat{\sigma}_{\hat{\mu}} .
$$

In this equation, $\hat{\mu}$ is the arithmetic mean of the data, $\hat{\sigma}_{\hat{\mu}}$ is the estimate of the standard deviation of the mean, and $\mathrm{t}_{\mathrm{df}, 0.05\rangle}$ is the quantile from Student's $\mathbf{t}$ distribution with df degrees of freedom for a one-sided $95 \%$ confidence interval.

For the tank 241-U-108 data (per sample number), df equals the number of observations minus one; i.e., $\mathrm{df}=1$.

The upper limit of the 95 percent confidence interval for each sample number based on alpha data is listed in Table C1-1. Each confidence interval can be used to make the following statement. If the upper limit is less than $41 \mu \mathrm{Ci} / \mathrm{g}(61.5 \mu \mathrm{Ci} / \mathrm{mL}$ for drainable liquid), then one would reject the null hypothesis that the alpha is greater than or equal to $41 \mu \mathrm{Ci} / \mathrm{g}$ $(61.5 \mu \mathrm{Ci} / \mathrm{mL}$ for drainable liquid) at the 0.05 level of significance.

The upper limit of the 95 percent confidence interval for each sample number based on DSC data is listed in Table C1-2. Each confidence interval can be used to make the following statement. If the upper limit is less than $480 \mathrm{~J} / \mathrm{g}$, then one would reject the null hypothesis that DSC is greater than or equal to $480 \mathrm{~J} / \mathrm{g}$ at the 0.05 level of significance. 
Table C1-1. 95 Percent Confidence Interval Upper Limits for Alpha for Tank 241-U-108 (Units are $\mu \mathrm{Ci} / \mathrm{g}$ or $\mu \mathrm{Ci} / \mathrm{mL}$ ). (2 sheets)

\begin{tabular}{|c|c|c|c|c|}
\hline 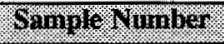 & Sanplo Boratiolion. & \% & $6 \%$ & (y) \\
\hline \$96T002257 & Core 141 , segment 1 , lower half & 0.080 & 0.013 & 0.163 \\
\hline S96T002258 & Core 141 , segment 2 , upper half & 0.032 & 0.004 & 0.054 \\
\hline S96T002259 & Core 141 , segment 3 , upper half & 0.029 & 0.002 & 0.041 \\
\hline S96T002260 & Core 141 , segment $3 \mathrm{~A}$, upper half & 0.026 & 0.003 & 0.044 \\
\hline S96T002261 & Core 141 , segment 4 , upper half & 0.051 & 0.005 & 0.084 \\
\hline S96T002262 & Core 141, segment 4, lower half & 0.040 & 0.003 & 0.059 \\
\hline S96T002263 & Core 141 , segment $4 A$, upper half & 0.053 & 0.001 & 0.056 \\
\hline S96T002264 & Core 141 , segment $4 \mathrm{~A}$, lower half & $0 . \overline{031}$ & 0.002 & 0.042 \\
\hline S96T0022821 & Core 141 , segment 1 , drainable liquid & $0 . \overline{008}$ & 0.001 & 0.012 \\
\hline S96T002608 & Core 141, segment 5 , upper half & 0.056 & 0.004 & 0.082 \\
\hline S96T002609 & Core 141 , segment 5 , lower half & 0.048 & 0.003 & 0.065 \\
\hline S96T002610 & Core 141 , segment 6 , upper half & 0.032 & 0.004 & 0.059 \\
\hline S96T002611 & Core 141, segment 6 , lower half & 0.016 & 0.004 & 0.040 \\
\hline S96T002612 & Core 141 , segment 7 , upper half & 0.035 & 0.003 & 0.057 \\
\hline S96T002613 & Core 141 , segment 7 , lower half & 0.029 & $3.50 \mathrm{E}-04$ & 0.031 \\
\hline S96T0026142 & Core 141 , segment 8 , upper half & 0.038 & 0.034 & 0.250 \\
\hline S96T002615 & Core 141 , segment 8 , lower half & 0.110 & 0.001 & 0.113 \\
\hline \$96T002616 & Core 141 , segment 9 , upper half & 0.043 & 0.006 & 0.080 \\
\hline S96T002909 & Core 145 , segment 2 , upper half & 0.027 & 0.000 & 0.028 \\
\hline S96T002910 & Core 145 , segment 3 , upper half & 0.042 & 0.001 & 0.047 \\
\hline S96T002911 & Core 145 , segment 3 , lower half & 0.040 & 0.003 & 0.057 \\
\hline S96T002912 & Core 145 , segment 4 , upper half & 0.033 & 0.002 & 0.044 \\
\hline S96T002913 & Core 145 , segment 4 , lower half & 0.047 & $4.00 \mathrm{E}-04$ & 0.049 \\
\hline S96T002914 & Core 145 , segment 5 , upper half & 0.037 & 0.002 & 0.051 \\
\hline S96T002915 & Core 145 , segment 5 , lower half & 0.032 & 0.001 & 0.040 \\
\hline S96T002916 & Core 145 , segment 6 , upper half & 0.017 & 0.003 & 0.033 \\
\hline S96T002917 & Core 145 , segment 6 , lower half & 0.014 & 0.001 & 0.019 \\
\hline S96T002918 & Core 145 , segment 7 , upper half & 0.017 & 0.001 & 0.023 \\
\hline \$96T002919 & Core 145 , segment 7 , lower half & 0.021 & $5.00 \mathrm{E}-05$ & 0.022 \\
\hline S96T002920 & Core 145 , segment 8 , upper half & 0.013 & $5.00 \mathrm{E}-05$ & 0.013 \\
\hline S96T002921 & Core 145 , segment 8 , lower half & 0.031 & $2.50 \mathrm{E}-04$ & 0.033 \\
\hline S96T002922 & Core 145 , segment 9 , upper half & 0.254 & 0.009 & 0.307 \\
\hline S96T002923 & Core 145 , segment 9 , lower half & 0.063 & 0.008 & 0.116 \\
\hline
\end{tabular}


Table C1-1. 95 Percent Confidence Interval Upper Limits for Alpha for Tank 241-U-108 (Units are $\mu \mathrm{Ci} / \mathrm{g}$ or $\mu \mathrm{Ci} / \mathrm{mL}$ ). (2 sheets)

\begin{tabular}{|c|c|c|c|c|}
\hline 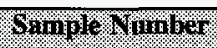 & Samplo beseription & 4. & 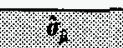 & W1. \\
\hline S96T002942 & Core 145 , segment 1 , drainable liquid & 0.011 & $4.50 \mathrm{E}-04$ & 0.014 \\
\hline S96T003001 & Core 146 , segment $3 A$, upper half & 0.029 & $2.00 \mathrm{E}-04$ & 0.030 \\
\hline S96T003002 & Core 146 , segment 4 , upper half & 0.039 & $4.00 \mathrm{E}-04$ & 0.042 \\
\hline S96T003003 & Core 146 , segment 4 , lower half & 0.102 & 0.010 & 0.167 \\
\hline S96T003004 & Core 146 , segment 5 , upper half & 0.090 & 0.008 & 0.140 \\
\hline S96T003005 & Core 146 , segment 5 , lower half & $0.05 \overline{3}$ & 0.007 & 0.098 \\
\hline S96T003006 $6^{1,2}$ & Core 146 , segment 6 , upper half & 0.045 & 0.013 & 0.126 \\
\hline S96T003007 $7^{1,2}$ & Core 146 , segment 6 , lower half & 0.109 & 0.017 & 0.215 \\
\hline S96T003008 & Core 146 , segment 7 , upper half & 0.019 & 0.001 & 0.024 \\
\hline S96T003009 & Core 146 , segment 7 , lower half & 0.028 & 0.003 & 0.046 \\
\hline S96T003143 & Core 146 , segment 1 , upper half & 0.039 & $3.50 \mathrm{E}-04$ & 0.041 \\
\hline S96T003144 & Core 146 , segment 2 , upper half & 0.026 & 0.001 & 0.032 \\
\hline S96T003145 & Core 146 , segment 2 , lower half & 0.012 & 0.001 & 0.016 \\
\hline$\overline{\text { S96T003146 }}$ & Core 146 , segment 3 , upper half & 0.033 & $1.00 \mathrm{E}-04$ & 0.034 \\
\hline S96T003147 & Core 146 , segment 3 , lower half & 0.011 & $3.50 \mathrm{E}-04$ & 0.013 \\
\hline S96T003148 & Core 146 , segment 8 , upper half & 0.024 & 0.002 & 0.036 \\
\hline S96T003149 & Core 146 , segment 8 , lower half & $0 . \overline{045}$ & 0.006 & 0.085 \\
\hline S96T003150 & Core 146, segment 9, First Quarter & 0.177 & 0.006 & 0.211 \\
\hline S96T003163 1,2 & Core 146 , segment 1 , drainable liquid & 0.010 & 0.000 & 0.010 \\
\hline S96T004180 & Core 146 , segment 9 , lower half & 0.364 & 0.001 & 0.370 \\
\hline
\end{tabular}

Notes:

${ }^{1}$ Sample result is below the detection limit.

${ }^{2}$ Duplicate result is below the detection limit. 
Table C1-2. 95 Percent Confidence Interval Upper Limits for DSC for Tank 241-U-108 (Units are J/g Dry).

\begin{tabular}{|c|c|c|c|c|}
\hline Sanvele Nomber & Shample Deserrintion. & 2 & c. & (1) \\
\hline S96T002241 & Core 141 , segment 1 , lower half & 180.0 & 24.0 & 331.5 \\
\hline S96T002242 & Core 141 , segment 2 , upper half & 61.1 & 1.6 & 71.2 \\
\hline S96T002245 & Core 141 , segment 4 , upper half & 289.3 & 128.1 & 590.8 \\
\hline S96T002246 & Core 141 , segment 4 , lower half & 221.7 & 65.0 & 411.6 \\
\hline S96T002589 & Core 141 , segment 7 , upper half & 92.4 & 32.7 & 298.5 \\
\hline S96T002590 & Core 141 , segment 7 , lower half & 211.5 & 96.5 & 820.8 \\
\hline S96T002591 & Core 141 , segment 8 , upper half & 148.0 & 9.3 & 175.1 \\
\hline S96T002592 & Core 141 , segment 8 , lower half & 127.5 & 8.5 & 181.2 \\
\hline S96T002879 & Core 145 , segment 3 , upper half & 195.0 & 14.0 & 283.4 \\
\hline S96T002880 & Core 145 , segment 3 , lower half & 164.0 & 1.0 & 170.3 \\
\hline S96T002881 & Core 145 , segment 4 , upper half & 131.5 & 16.7 & 180.3 \\
\hline S96T002884 & Core 145 , segment 5 , lower half & 28.6 & 2.4 & 43.4 \\
\hline S96T002885 & Core 145 , segment 6 , upper half & 49.5 & 33.0 & 145.8 \\
\hline S96T002886 & Core 145 , segment 6 , lower half & 77.7 & 77.7 & 304.5 \\
\hline S96T002887 & Core 145 , segment 7 , upper half & 191.5 & 61.5 & 579.8 \\
\hline S96T002888 & Core 145 , segment 7 , lower half & 169.5 & 1.5 & 179.0 \\
\hline S96T002891 & Core 145 , segment 9 , upper half & 108.5 & 3.5 & 130.6 \\
\hline S96T002892 & Core 145, segment 9, lower half & 74.0 & 30.0 & 263.4 \\
\hline S96T002942 & Core 145 , segment 1 , drainable liquid & 156.0 & 1.0 & 162.3 \\
\hline S96T002960 & Core 146 , segment 4 , upper half & 20.7 & 20.7 & 151.4 \\
\hline S96T002961 & Core 146 , segment 4 , lower half & 104.0 & 2.0 & 116.6 \\
\hline S96T002962 & Core 146 , segment 5 , upper half & 155.0 & 4.0 & 180.3 \\
\hline S96T002963 & Core 146 , segment 5 , lower half & 145.0 & 11.0 & 214.5 \\
\hline S96T002964 & Core 146 , segment 6 , upper half & 36.2 & 8.8 & 91.4 \\
\hline S96T002967 & Core 146 , segment 7 , lower half & 116.5 & 7.5 & 163.9 \\
\hline S96T003121 & Core 146 , segment 1 , upper half & 114.0 & 5.0 & 145.6 \\
\hline S96T003124 & Core 146 , segment 2 , lower half & 78.7 & $9 . \overline{6}$ & 138.9 \\
\hline S96T003125 & Core 146 , segment 3 , upper half & 44.2 & 27.0 & 214.7 \\
\hline S96T003126 & Core 146 , segment 3 , lower half & 2.4 & 2.4 & 17.3 \\
\hline S96T003129 & Core 146, segment 9, First Quarter & 92.1 & 1.6 & 102.2 \\
\hline S96T003163 & Core 146 , segment 1 , drainable liquid & 170.0 & 11.0 & 239.5 \\
\hline
\end{tabular}




\section{C1.1 STATISTICS FOR THE ORGANIC DQO}

The organic DQO (Turner et al. 1996) defines acceptable decision confidence limits in terms of one-sided 95 percent confidence intervals. In this appendix, one-sided confidence limits supporting the organic DQO are calculated for tank 241-U-108. All data considered in this section are taken from the final laboratory data package for the 1996 core sampling event for tank 241-U-108 (Bell 1996).

Confidence intervals were computed for each sample number from tank 241-U-108 analytical data. The sample numbers and confidence intervals are provided in Table C1-3 for percent water and table C1-4 for TOC.

For percent water, the lower limit (LL) of a one-sided 95 percent confidence interval for the mean is

$$
\hat{\mu}-\mathrm{t}_{(\mathrm{df}, 0.05)} * \hat{\sigma}_{\hat{\mu}}
$$

and for TOC, the upper limit (UL) of a one-sided 95 percent confidence interval for the mean is

$$
\hat{\mu}+t_{(d f, 0.05)} * \hat{\sigma}_{\hat{\mu}} \text {. }
$$

For these equations, $\hat{\mu}$ is the arithmetic mean of the data, $\hat{\sigma}_{\hat{\mu}}$ is the estimate of the standard deviation of the mean, and $t_{(d f, 0.05)}$ is the quantile from Student's $t$ distribution with df (the number of observations minus one) degrees of freedom for a one-sided $95 \%$ confidence interval. In this case, df $=1$.

The lower limit of the 95 percent confidence interval for each sample number based on percent water data is listed in Table C1-3. Each confidence interval can be used to make the following statement. If the lower limit is greater than 17 percent, then one would reject the null hypothesis that the percent water is less than or equal to 17 percent at the 0.05 level of significance.

The upper limit of the 95 percent confidence interval for each sample number based on TOC data is listed in Table C1-4. Each confidence interval can be used to make the following statement: "If the upper limit is less than $30,000 \mu \mathrm{g} / \mathrm{g}$, then one would reject the null hypothesis that TOC is greater than or equal to $30,000 \mu \mathrm{g} / \mathrm{g}$ at the 0.05 level of significance." 
Table C1-3. 95 Percent Confidence Interval Lower Limits for Percent Water for Tank 241-U-108 (Units are in \%). (2 sheets)

\begin{tabular}{|c|c|c|c|c|}
\hline 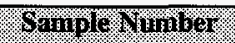 & 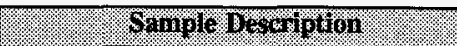 & 10. & 8 & Sil. \\
\hline S95T000978 & Riser 7, Grab Sample & 50.28 & 0.07 & 49.80 \\
\hline S96T002241 & Core 141 , segment 1 , lower half & 45.50 & 0.42 & 42.85 \\
\hline \$96T002242 & Core 141 , segment 2 , upper half & 23.74 & 0.69 & 19.38 \\
\hline S96T002243 & Core 141 , segment 3 , upper half & 30.26 & 3.28 & 9.55 \\
\hline S96T002244 & Core 141 , segment $3 \mathrm{~A}$, upper half & 25.74 & 6.30 & 0.00 \\
\hline S96T002245 & Core 141 , segment 4 , upper half & 32.81 & 0.24 & 31.32 \\
\hline S96T002246 & Core 141 , segment 4 , lower half & 29.89 & 1.30 & 21.68 \\
\hline S96T002247 & Core 141 , segment $4 A$, upper half & 29.98 & 14.50 & 0.00 \\
\hline S96T002248 & Core 141 , segment $4 \mathrm{~A}$, lower half & 39.10 & 0.37 & 36.79 \\
\hline S96T002282 & Core 141 , segment 1 , drainable liquid & 49.21 & 1.88 & 37.37 \\
\hline S96T002585 & Core 141 , segment 5 , upper half & 27.01 & 2.16 & 13.37 \\
\hline S96T002586 & Core 141 , segment 5 , lower half & 32.07 & 3.67 & 8.90 \\
\hline S96T002587 & Core 141 , segment 6 , upper half & 22.19 & 0.87 & 16.70 \\
\hline S96T002588 & Core 141 , segment 6 , lower half & 18.45 & 2.77 & 0.99 \\
\hline S96T002589 & Core 141 , segment 7 , upper half & 38.86 & 0.66 & 34.69 \\
\hline S96T002590 & Core 141 , segment 7 , lower half & 40.23 & 0.08 & 39.75 \\
\hline S96T002591 & Core 141 , segment 8 , upper half & 40.71 & 1.34 & 36.81 \\
\hline S96T002592 & Core 141 , segment 8 , lower half & 39.61 & 1.71 & 28.84 \\
\hline S96T002593 & Core 141 , segment 9 , upper half & 32.32 & 1.56 & 22.47 \\
\hline S96T002879 & Core 145 , segment 3 , upper half & 52.20 & 9.04 & 25.80 \\
\hline S96T002880 & Core 145 , segment 3 , lower half & 45.77 & 0.21 & 44.44 \\
\hline S96T002881 & Core 145 , segment 4 , upper half & 40.97 & 1.01 & 34.62 \\
\hline S96T002882 & Core 145 , segment 4 , lower half & 25.72 & 0.16 & 24.71 \\
\hline S96T002883 & Core 145 , segment 5 , upper half & 17.73 & 0.43 & 15.04 \\
\hline S96T002884 & Core 145 , segment 5 , lower half & 30.94 & 2.53 & 14.99 \\
\hline S96T002885 & Core 145 , segment 6 , upper half & 28.09 & 0.59 & 24.39 \\
\hline S96T002886 & Core 145 , segment 6 , lower half & 34.14 & 4.75 & 4.15 \\
\hline S96T002887 & Core 145 , segment 7 , upper half & 44.15 & 1.70 & 33.42 \\
\hline S96T002888 & Core 145 , segment 7, lower half & 42.29 & 0.05 & 42.00 \\
\hline S96T002889 & Core 145 , segment 8 , upper half & 42.21 & 0.71 & 37.73 \\
\hline S96T002890 & Core 145 , segment 8 , lower half & 40.61 & 0.57 & 37.04 \\
\hline S96T002891 & Core 145 , segment 9 , upper half & 39.22 & 0.88 & 33.66 \\
\hline S96T002892 & Core 145 , segment 9 , lower half & 49.27 & 0.41 & 46.71 \\
\hline
\end{tabular}


Table C1-3. 95 Percent Confidence Interval Lower Limits for Percent Water for Tank 241-U-108 (Units are in \%). (2 sheets)

\begin{tabular}{|c|c|c|c|c|}
\hline Sanple Ninior & Shinple Werowplon & 1) & 6 & Yis \\
\hline S96T002893 & Core 145 , segment 2 , upper half & 33.16 & 1.26 & 25.20 \\
\hline S96T002942 & Core 145 , segment 1 , drainable liquid & 49.46 & 0.21 & 48.16 \\
\hline S96T002959 & Core 146 , segment $3 \mathrm{~A}$, upper half & 20.18 & 2.94 & 1.62 \\
\hline S96T002960 & Core 146 , segment 4 , upper half & 17.55 & 0.34 & 15.40 \\
\hline S96T002961 & Core 146 , segment 4 , lower half & 40.83 & 0.51 & 37.61 \\
\hline S96T002962 & Core 146 , segment 5 , upper half & 38.93 & 2.21 & 25.00 \\
\hline S96T002963 & Core 146 , segment 5 , lower half & 34.78 & 3.61 & 12.01 \\
\hline S96T002964 & Core 146 , segment 6 , upper half & 32.42 & 2.94 & 13.88 \\
\hline S96T002965 & Core 146 , segment 6 , lower half & 44.96 & 0.66 & 40.82 \\
\hline S96T002966 & Core 146 , segment 7 , upper half & 33.43 & 0.13 & 32.64 \\
\hline S96T002967 & Core 146 , segment 7 , lower half & 41.05 & 0.09 & 40.48 \\
\hline S96T003121 & Core 146 , segment 1 , upper half & 39.75 & 2.05 & 26.83 \\
\hline S96T003123 & Core 146 , segment 2 , upper half & 26.59 & 2.93 & 8.12 \\
\hline S96T003124 & Core 146 , segment 2 , lower half & 12.27 & 2.68 & 0.00 \\
\hline S96T003125 & Core 146 , segment 3 , upper half & 35.53 & 6.04 & 0.00 \\
\hline S96T003126 & Core 146 , segment 3 , lower half & 20.42 & 1.22 & 12.74 \\
\hline S96T003127 & Core 146 , segment 8 , upper half & 41.27 & 0.02 & 41.17 \\
\hline S96T003128 & Core 146 , segment 8 , lower half & 40.23 & 0.16 & 39.22 \\
\hline S96T003129 & Core 146, segment 9, First Quarter & 38.54 & 0.08 & 38.03 \\
\hline S96T003163 & Core 146 , segment 1 , drainable liquid & 51.13 & 0.02 & 51.00 \\
\hline S96T004178 & Core 146 , segment 9 , lower half & 48.21 & 0.38 & 45.81 \\
\hline
\end{tabular}


Table C1-4. 95 Percent Confidence Interval Upper Limits for TOC for Tank

241-U-108 (Units are in $\mu \mathrm{g} / \mathrm{g}$ Dry). (2 sheets)

\begin{tabular}{|c|c|c|c|c|}
\hline Gamplo lowimer. & . & th. & 8 & U1. \\
\hline S96T002241 & Core 141 , segment 1 , lower half & 11523 & 514 & 14767 \\
\hline S96T002242 & Core 141 , segment 2 , upper half & 4281 & 269 & 5979 \\
\hline S96T002243 & Core 141 , segment 3 , upper half & 3829 & 14 & 3919 \\
\hline S96T002244 & Core 141 , segment $3 \mathrm{~A}$, upper half & 3993 & 20 & 4120 \\
\hline S96T002245 & Core 141 , segment 4 , upper half & 5844 & 879 & 8409 \\
\hline \$96T002246 & Core 141 , segment 4 , lower half & 6918 & 143 & 7818 \\
\hline S96T002247 & Core 141 , segment $4 \mathrm{~A}$, upper half & 6427 & 86 & 6968 \\
\hline S96T002248 & Core 141 , segment $4 \mathrm{~A}$, lower half & 7323 & 181 & 8463 \\
\hline S96T002282 & Core 141 , segment 1 , drainable liquid & 9720 & 82 & 10240 \\
\hline S96T002585 & Core 141, segment 5 , upper half & 6357 & 356 & 8606 \\
\hline S96T002586 & Core 141 , segment 5 , lower half & $5 \overline{653}$ & 15 & 5746 \\
\hline S96T002587 & Core 141 , segment 6 , upper half & 4280 & 64 & 4685 \\
\hline S96T002588 & Core 141 , segment 6 , lower half & 3452 & 264 & 5116 \\
\hline S96T002589 & Core 141 , segment 7 , upper half & 10329 & 384 & 12756 \\
\hline S96T002590 & Core 141 , segment 7 , lower half & 8808 & 8 & 8861 \\
\hline S96T002591 & Core 141 , segment 8 , upper half & 9825 & 582 & 13499 \\
\hline S96T002592 & Core 141 , segment 8 , lower half & 7556 & 963 & 10368 \\
\hline S96T002593 & Core 141 , segment 9 , upper half & 3635 & 251 & 5221 \\
\hline S96T002879 & Core 145 , segment 3 , upper half & 6652 & 21 & 6784 \\
\hline S96T002880 & Core 145 , segment 3 , lower half & 6011 & 369 & 8340 \\
\hline S96T002881 & Core 145 , segment 4 , upper half & 4972 & 25 & 5132 \\
\hline S96T002882 & Core 145 , segment 4 , lower half & 5203 & 7 & 5246 \\
\hline S96T002883 & Core 145, segment 5 , upper half & 4509 & 12 & 4586 \\
\hline S96T002884 & Core 145 , segment 5 , lower half & 8043 & 36 & 8272 \\
\hline S96T002885 & Core 145 , segment 6 , upper half & 6793 & 49 & 7100 \\
\hline S96T002886 & Core 145 , segment 6 , lower half & 6233 & 190 & 7431 \\
\hline S96T002887 & Core 145 , segment 7 , upper half & 8245 & 188 & 9432 \\
\hline S96T002888 & Core 145 , segment 7 , lower half & 9183 & 104 & 9839 \\
\hline S96T002889 & Core 145 , segment 8 , upper half & 5001 & 17 & 5110 \\
\hline S96T002890 & Core 145 , segment 8 , lower half & 7728 & 101 & 8366 \\
\hline S96T002891 & Core 145 , segment 9 , upper half & 8588 & 214 & 9939 \\
\hline S96T002892 & Core 145 , segment 9 , lower half & 5509 & 128 & 6318 \\
\hline S96T002893 & Core 145 , segment 2 , upper half & 4017 & 112 & 4726 \\
\hline
\end{tabular}


Table C1-4. 95 Percent Confidence Interval Upper Limits for TOC for Tank 241-U-108 (Units are in $\mu \mathrm{g} / \mathrm{g}$ Dry). (2 sheets)

\begin{tabular}{|l|l|r|r|r|}
\hline S96T002893 & Core 145, segment 2, upper half & 4017 & 112 & 4726 \\
\hline S96T002942 & Core 145, segment 1, drainable liquid & 10014 & 35 & 10239 \\
\hline S96T002959 & Core 146, segment 3A, upper half & 3875 & 526 & 5410 \\
\hline S96T002960 & Core 146, segment 4, upper half & 3178 & 24 & 3331 \\
\hline S96T002961 & Core 146, segment 4, lower half & 7115 & 101 & 7755 \\
\hline S96T002962 & Core 146, segment 5, upper half & 7966 & 352 & 10188 \\
\hline S96T002963 & Core 146, segment 5, lower half & 6271 & 368 & 8594 \\
\hline S96T002964 & Core 146, segment 6, upper half & 6214 & 30 & 6401 \\
\hline S96T002965 & Core 146, segment 6, lower half & 8057 & 9 & 8114 \\
\hline S96T002966 & Core 146, segment 7, upper half & 8044 & 593 & 11790 \\
\hline S96T002967 & Core 146, segment 7, lower half & 11654 & 950 & 17652 \\
\hline S96T003121 & Core 146, segment 1, upper half & 6572 & 66 & 6991 \\
\hline S96T003123 & Core 146, segment 2, upper half & 3821 & 20 & 3950 \\
\hline S96T003124 & Core 146, segment 2, lower half & 1989 & 40 & 2241 \\
\hline S96T003125 & Core 146, segment 3, upper half & 4291 & 163 & 4766 \\
\hline S96T003126 & Core 146, segment 3, lower half & 2940 & 187 & 3485 \\
\hline S96T003127 & Core 146, segment 8, upper half & 9756 & 451 & 11074 \\
\hline S96T003128 & Core 146, segment 8, lower half & 5504 & 100 & 6138 \\
\hline S96T003129 & Core 146, segment 9, upper half & 18142 & 81 & 18656 \\
\hline S96T003163 & Core 146, segment 1, drainable liquid & 10505 & 199 & 11765 \\
\hline S96T004178 & Core 146, segment 9, lower half & 10349 & 348 & 12544 \\
\hline
\end{tabular}




\section{C2.0 APPENDIX C REFERENCES}

Bell, K. E., 1996, Final Report for Tank 241-U-108, Push-Mode Core Samples 141, 145, and 146, WHC-SD-WM-DP-198, Rev. 0, Westinghouse Hanford Company, Richland, Washington.

Dukelow, G. T., J. W. Hunt, H. Babad, and J. E. Meacham., 1995, Tank Safety Screening Data Quality Objective, WHC-SD-WM-SP-004, Rev. 2, Westinghouse Hanford Company, Richland, Washington.

Turner, D. A., H. Babad, L. L. Buckley, J. E. Meacham, 1996, Data Quality Objective to Support Resolution of the Organic Complexant Safety Issue, WHC-SD-WM-DQO-006, Rev. 2, Westinghouse Hanford Company, Richland, Washington. 
HNF-SD-WM-ER-639 Rev. 0

\section{APPENDIX D}

EVALUATION TO ESTABLISH BEST-BASIS

INVENTORY FOR TANK 241-U-108 
HNF-SD-WM-ER-639 Rev. 0

This page left blank intentionally. 


\section{APPENDIX D \\ EVALUATION TO ESTABLISH BEST-BASIS INVENTORY FOR TANK 241-U-108}

An effort is underway to provide waste inventory estimates that will serve as standard characterization information for the various waste management activities (Kupfer et al. 1995). As part of this effort, an evaluation of available chemical information for tank 241-U-108 was performed, and a best-basis inventory was established. This work, detailed in the following sections, follows the methodology that was established by the standard inventory task.

\section{D1.0 CHEMICAL INFORMATION SOURCES}

Available chemical information for tank 241-U-108 included:

- Data from recent analyses of three core samples collected in April/May 1996 (Bell 1996). See Appendix B, Section B2.0 for data.

- The solids composite inventory estimate for this tank generated from the Hanford Defined Waste (HDW) model (Agnew et al. 1996a), developed at Los Alamos National Laboratory.

A list of references used in this evaluation is provided at the end of this Appendix.

\section{D2.0 COMPARISON OF COMPONENT INVENTORY VALUES}

The sample-based inventory estimate (Section B3.2), derived from the analytical concentration data from the three 1996 core samples, and the inventory estimate from the HDW (Section A3.2), are compared in Tables D2-1 and D2-2. The HDW provides tank content estimates derived from process flowsheets and waste volume records. The waste volume used to generate both estimates is $1,771 \mathrm{~kL}$ ( $468 \mathrm{kgal})$. However, the sample- and HDW-based estimates use different waste densities. The sample-based inventory was generated using a measured bulk density of $1.74 \mathrm{~g} / \mathrm{mL}$ for segment sample data and $1.40 \mathrm{~g} / \mathrm{mL}$ for drainable liquid samples, and a density of $1.71 \mathrm{~g} / \mathrm{mL}$ for core composites. The current HDW shows a lower waste density of $1.62 \mathrm{~g} / \mathrm{mL}$. The differences attributable to density result in a relative percent difference of 7.1 for analytes with roughly the same concentration. 
The sample-based values in Table D2-1 were obtained either from the mass-weighted average of the solid segment data (Table B3-9) plus the drainable liquid data (Table B3-10), or from the core composite results (Table B3-8) as indicated. The segment/drainable liquid data were used preferentially because they provided a more comprehensive description of the tank. The component inventories were calculated by multiplying the mean analyte concentration value by the current tank waste volume and the appropriate density of the waste.

There are several differences between the sample-based and HDW inventories for some analytes. Analyte inventories that vary by about a factor of two between the two bases are $\mathrm{Al}$, phosphate, $\mathrm{Cr}$, and carbonate. Analytes that vary by an order of magnitude or more are $\mathrm{La}$ and $\mathrm{U}$. Of the radionuclides, $\mathrm{Sr}-90$ is reported to be about an order of magnitude less in the sampling inventory than in the HDW.

Table D2-1. Sampling and HDW-Based Inventory Estimates for Nonradioactive Components in Tank 241-U-108. (2 sheets)

\begin{tabular}{|c|c|c|c|c|c|}
\hline (6). & 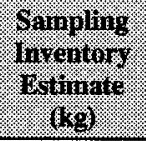 & 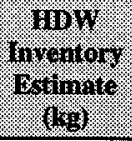 & k. & 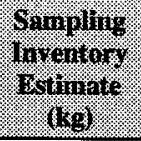 & 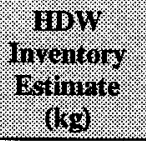 \\
\hline $\mathrm{Al}$ & $51,190^{1}$ & 96,800 & $\mathrm{NH}_{3}$ & $\mathrm{n} / \mathrm{r}$ & 1,790 \\
\hline $\mathrm{Ag}$ & $34.1^{2}$ & $\mathrm{n} / \mathrm{r}$ & $\mathrm{Ni}$ & 150 & 734 \\
\hline As & $<234$ & $n / r$ & $\mathrm{NO}_{2}^{-}$ & $157,800^{1}$ & 210,000 \\
\hline B & 239 & $\mathrm{n} / \mathrm{r}$ & $\mathrm{NO}_{3}^{-}$ & $879,500^{1}$ & 594,000 \\
\hline $\mathrm{Ba}$ & $<117$ & $\mathrm{n} / \mathrm{r}$ & oxalate & $12,700^{1}$ & 5.63 \\
\hline $\mathrm{Be}$ & $<11.7$ & $\mathrm{n} / \mathrm{r}$ & $\mathrm{Pb}$ & $<234$ & 7,020 \\
\hline $\mathrm{Bi}$ & $<5,930^{1}$ & 436 & $\mathrm{P}$ as $\mathrm{PO}_{4}{ }^{3-}$ & $50,527^{1}$ & 16,800 \\
\hline $\mathrm{Ca}$ & $447^{3}$ & 3,940 & $\mathrm{Sb}$ & $<152$ & $\mathrm{n} / \mathrm{r}$ \\
\hline $\mathrm{Cr}$ & $12,116^{1}$ & 5,980 & $\mathrm{Se}$ & $<234$ & $\mathrm{n} / \mathrm{r}$ \\
\hline $\mathrm{Ce}$ & $<234$ & $\mathrm{n} / \mathrm{r}$ & $\mathrm{S}$ as $\mathrm{SO}_{4}{ }^{2-}$ & $44,430^{1}$ & 45,000 \\
\hline $\mathrm{Co}$ & $<46.8$ & $\mathrm{n} / \mathrm{r}$ & $\mathrm{Si}$ & 830 & 4330 \\
\hline $\mathrm{Cl}$ & $10,914^{1}$ & 13,800 & $\mathrm{Sr}$ & $<23.4$ & 2.19 \\
\hline $\mathrm{Cd}$ & 12.8 & $\mathrm{n} / \mathrm{r}$ & $\begin{array}{l}\mathrm{TIC} \text { as } \\
\mathrm{CO}_{3}{ }^{2-}\end{array}$ & $118,500^{1}$ & 54,900 \\
\hline $\mathrm{Cu}$ & $<23.8$ & $\mathrm{n} / \mathrm{r}$ & $\mathrm{Te}$ & $\mathrm{n} / \mathbf{r}$ & $\mathrm{n} / \mathrm{r}$ \\
\hline $\mathrm{F}$ & $2,836^{1}$ & 2,230 & $\mathrm{Ti}$ & $<23.4$ & $\mathrm{n} / \mathrm{r}$ \\
\hline
\end{tabular}


Table D2-1. Sampling and HDW-Based Inventory Estimates for Nonradioactive Components in Tank 241-U-108. (2 sheets)

\begin{tabular}{|c|c|c|c|c|c|}
\hline \%). & 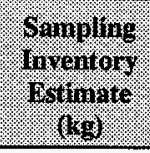 & 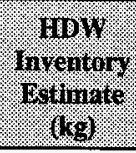 & 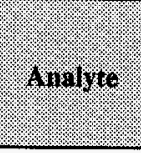 & 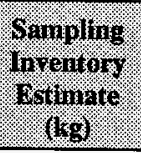 & 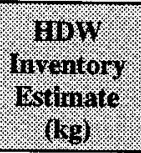 \\
\hline $\mathrm{Fe}$ & 507 & 3,390 & TOC & $12,850^{1}$ & $\mathrm{n} / \mathrm{r}$ \\
\hline $\mathrm{Hg}$ & $\mathrm{n} / \mathrm{r}$ & 221 & $\mathrm{U}_{\text {TOTAL }}$ & $538^{4}$ & 22,400 \\
\hline $\mathrm{K}$ & 4,800 & 4,100 & V & $<117$ & $\mathrm{n} / \mathrm{r}$ \\
\hline $\mathrm{La}$ & $<117$ & 10.4 & W & $\mathrm{n} / \mathrm{r}$ & $\mathrm{n} / \mathrm{r}$ \\
\hline $\mathrm{Mg}$ & $<234$ & $\mathrm{n} / \mathrm{r}$ & $\mathrm{Zn}$ & 131 & $\mathrm{n} / \mathrm{r}$ \\
\hline Mn & 185 & 435 & $\mathrm{Zr}$ & $<23.4$ & 129 \\
\hline Mo & 177 & $\mathrm{n} / \mathrm{r}$ & $\mathrm{H}_{2} \mathrm{O}(\mathrm{Wt} \%)$ & 34.3 & 33.0 \\
\hline $\mathrm{Na}$ & $726,100^{1}$ & 513,000 & \multirow{2}{*}{$\begin{array}{c}\text { Density } \\
(\mathrm{kg} / \mathrm{L})\end{array}$} & \multirow[t]{2}{*}{1.71} & \multirow[t]{2}{*}{1.62} \\
\hline Nd & $<234$ & $\mathrm{n} / \mathrm{r}$ & & & \\
\hline
\end{tabular}

Notes:

${ }^{1}$ Average of solid segment data plus liquid segment data.

${ }^{2}$ From liquid sample only.

${ }^{3}$ Acid digest value from core composite data.

${ }^{4}$ Total uranium value based on the three core composite averages using the laser phosphorescence analysis method on fusion digestions.

$<=$ "less than" values are average detection limits for analytical method used.

Table D2-2. Sampling and HDW Model-Based Inventory Estimates for Radioactive Components in Tank 241-U-108. (2 sheets)

\begin{tabular}{|c|c|c|c|c|c|}
\hline$y^{2}$ & 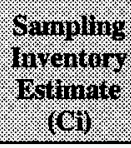 & 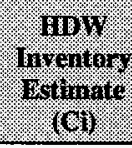 & Hnalige & 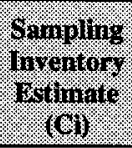 & 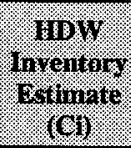 \\
\hline${ }^{14} \mathrm{C}$ & $\mathrm{n} / \mathrm{r}$ & $\mathrm{n} / \mathrm{r}$ & ${ }^{155} \mathrm{Eu}$ & $<884$ & $\mathrm{n} / \mathrm{r}$ \\
\hline${ }^{60} \mathrm{Co}$ & $<61.5$ & $\mathrm{n} / \mathrm{r}$ & ${ }^{237} \mathrm{~Np}$ & $\mathrm{n} / \mathrm{r}$ & $\mathrm{n} / \mathrm{r}$ \\
\hline${ }^{90} \mathrm{Sr}$ & $29,400^{1}$ & 243,000 & ${ }^{239 / 240} \mathrm{Pu}$ & $\mathrm{n} / \mathrm{r}$ & 11.0 \\
\hline
\end{tabular}


Table D2-2. Sampling and HDW Model-Based Inventory Estimates for Radioactive Components in Tank 241-U-108. (2 sheets)

\begin{tabular}{|c|c|c|c|c|c|}
\hline Analyte & 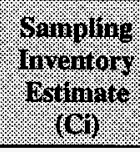 & 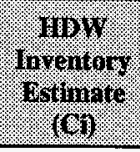 & Analye & 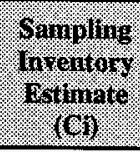 & 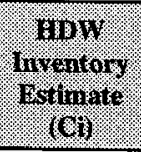 \\
\hline${ }^{99} \mathrm{Tc}$ & $\mathrm{n} / \mathrm{r}$ & $\mathrm{n} / \mathrm{r}$ & ${ }^{241} \mathrm{Am}$ & $<1930$ & $\mathrm{n} / \mathrm{r}$ \\
\hline${ }^{129} \mathrm{I}$ & $\mathrm{n} / \mathrm{r}$ & $n / r$ & Total $\alpha$ & 154 & $\mathrm{n} / \mathrm{r}$ \\
\hline${ }^{137} \mathrm{Cs}$ & $411,000^{1}$ & 463,000 & Total $\beta$ & $479,000^{2}$ & $\mathrm{n} / \mathrm{r}$ \\
\hline${ }^{154} \mathrm{Eu}$ & $<241$ & $\mathrm{n} / \mathrm{r}$ & & & \\
\hline
\end{tabular}

Notes:

${ }^{1}$ Average of solid segment data plus liquid segment data.

${ }^{2}$ Fusion digest value from core composite data.

\section{D3.0 COMPONENT INVENTORY EVALUATION}

The following evaluation of tank contents was performed in order to identify potential errors and/or missing information that could influence the sampling and/or the HDW-based component inventories.

\section{D3.1 CONTRIBUTING WASTE TYPES}

Tank 241-U-108 was the second tank in the 241-U-107, 241-U-108, and 241-U-109 cascade and it began receiving metal waste (MW) in the first quarter of 1949. In the mid 1950's, most of the MW was transferred out for uranium recovery.

From the second quarter of 1959 until the fourth quarter of 1964, the tank received REDOX cladding waste. Numerous transfers out of the tank took place during the 1960's. In the second quarter of 1964 , the tank again received cladding waste from the REDOX plant. It received a combination of $\mathrm{N}$-Reactor, REDOX cladding, and evaporator bottoms waste from the third quarter of 1968 until the first quarter of 1976. Some of these wastes were transferred out of tank 241-U-108 during the second quarter of 1972 and the fourth quarter of 1973 . From the second quarter of 1975 through the first quarter of 1977, the tank exchanged evaporator bottoms waste with tank 241-S-102. This was the final major transfer of waste involving tank 241-U-108.

Refer to Appendix A, Section A3.1 for a more detailed summary of the waste transfer history of tank $241-\mathrm{U}-108$. 
The types of solids accumulated in tank 241-U-108 during its history, as reported by various authors, are compiled in tables D3-1 and D3-2.

Table D3-1. Expected Solids for Tank 241-U-108.

\begin{tabular}{|l|c|}
\hline $\begin{array}{l}\text { (Anderson 1990) } \\
\text { SORWT Model (Hill et al. 1995) }\end{array}$ & $\begin{array}{c}\text { MW, REDOX CW, EB, N, BNW, DW, } \\
\text { HDRL, PNF, NCPLX }\end{array}$ \\
\hline WSTRS (Agnew et al. 1996b) & EB and CW mixture \\
\hline HDW Model (Agnew et al. 1996a) & MW, CW, EB, N, BNW, PNF, HDRL, \\
NCPLX
\end{tabular}

Notes:

$\begin{array}{lll}\text { MW } & = & \text { [Bismuth phosphate process U] metal waste } \\ \text { DW } & = & \text { Dilute waste } \\ \text { N } & = & \text { N-Reactor Waste } \\ \text { BNW } & = & \text { Battelle Northwest Laboratory Waste } \\ \text { HDRL } & = & \text { Hanford Defense Residual Liquor } \\ \text { NCPLX } & = & \text { Non-Complexed Waste } \\ \text { PNF } & = & \text { Partially Neutralized Waste } \\ \text { SMMS1 } & & \\ \text { and SMMS2 } & = & \text { Supernatant mixing model 242-S Evaporator waste. WSTRS Waste Status and } \\ \text { SORWT } & = & \text { Transaction Record Summary (Agnew et al. 1996b). }\end{array}$

The WSTRS document (Agnew et al. 1996b) as well as Anderson (1990) support the position that metal waste was removed from tank 241-U-108 before receipt of REDOX cladding wastes and evaporator bottoms. However, Appendix C and Appendix D of Agnew et al. (1996b) assign the sludge heel as $\mathrm{BiPO}_{4}$ metal waste.

\section{D3.2 EVALUATION OF PROCESS FLOWSHEET INFORMATION}

Detailed review of Agnew et al. (1996b) indicates that a considerable amount of transfer activity occurred with 241-U-108, but the dominant waste type currently is saltcake derived primarily from REDOX cladding wastes and evaporator bottoms. Essentially all the MW was removed during sluicing, but some traces of residual MW occur in the lower core sample segments. 
Table D3-2. HDW Solids for Tank 241-U-108.

\begin{tabular}{|c|c|c|}
\hline $\begin{array}{l}\text { Trail I layer Model Soltals } \\
\text { Layer. }\end{array}$ & MI & I.99: \\
\hline MW & 11.3 & 3 \\
\hline CWR2 & 98.4 & 26 \\
\hline SMMSI & 874.3 & 231 \\
\hline SMMS2 & 696.4 & 184 \\
\hline
\end{tabular}

${ }^{1}$ From Agnew et al (1996a)

\section{D3.2.1 Metal Wastes}

Although very little MW is estimated to be in tank $241-\mathrm{U}-108$ (3 kgal) there is a significant discrepancy in the uranium inventory between the HDW $(22,400 \mathrm{~kg})$ and the sample data $(538 \mathrm{~kg})$. Tank $241-\mathrm{U}-108$ has a dished bottom, and all cores were taken from the outer edges of the tank. Thus, a heel of MW would not have been sampled. Nevertheless, all cores exhibited an increase in phosphorous concentration in segment 9. Because of sampling location and an increase in phosphorous concentration in the lower segments, there is a possibility that undetected uranium inventory could be in the dished bottom. The uranium recovery manual and declassified sluicing records, as well as Agnew et al. (1996b) data, were used in an attempt to evaluate this discrepancy.

The uranium recovery waste (UR) manual lists approximate sludge levels $(1.2 \mathrm{~m}[4 \mathrm{ft}]$, $0.6 \mathrm{~m}[1.9 \mathrm{ft}], 0 \mathrm{~m}$ [0 ft], respectively) expected to develop for a three-tank cascade after transfer of uranium effluent from the bismuth phosphate process. For a dish-bottomed tank with a $23-\mathrm{m}$ (75-ft) diameter and the approximate sludge levels, it is estimated that 70 volume percent of the sludge was in the first tank and 30 volume percent was in the second. Declassified sluicing records for the 241-U-107, $-\mathrm{U}-108,-\mathrm{U}-109$ cascade indicate a total of 74 short tons of uranium remained in the total cascade after sluicing (Rodenhizer 1987). Assuming 30 percent of this inventory may have been left in tank 241-U-108 yields a value of $20,140 \mathrm{~kg}$ of uranium, which is comparable to the HDW value of $22,400 \mathrm{~kg}$. However, neither of these values are compatible with a uranium inventory of $4,957 \mathrm{~kg}$ obtained as a product of the HDW MW2 sludge concentration of $269.5 \mathrm{~g} / \mathrm{kg}$ and $11.4 \mathrm{~kL}$ (3 kgal) of waste at a HDW sludge density of $1.62 \mathrm{~kg} / \mathrm{L}$.

A uranium inventory of $<29,700 \mathrm{~kg}$ from average segment ICP-fusion data where all segments were below detection limits and uranium inventories varying from $<1,117 \mathrm{~kg}$ (acid digestion ICP) to $<27,600 \mathrm{~kg}$ (fusion digestion ICP) from core composite data also does not provide a basis for comparison because all results were below analytical detection limits. The laser phosphorescence data from core composite samples were all above detection limits and are considered the more reliable data. Because of the uncertainty associated with the sludge level estimates and remaining inventory after sluicing, as well as the inconsistency of 
the HDW estimates, and the relatively small amount of estimated MW, the sampling values for uranium in tank 241-U-108 obtained by laser-phosphorescence analytical methods were adopted.

\section{D3.2.2 REDOX Cladding Waste}

The WSTRS document (Agnew 1996b) indicates $749 \mathrm{~kL}$ (198 kgal) of CWR was received by tank $241-\mathrm{U}-108$ in 1964 and $2,839 \mathrm{~kL}$ (750 kgal) was received in 1968 . This latter transfer in 1968 occurred about a year after REDOX ceased operations, and may have represented supernatant from REDOX cladding waste. In 1964, tank 241-S-107 received about 1,366 kL (361 kgal) of CWR which, when combined with $198 \mathrm{kgal}$ of tank 241-U-108 waste in 1964, yields a total of $2,115 \mathrm{~kL}(559 \mathrm{kgal})$. The fraction of this total volume in tank $241-\mathrm{U}-108$ is 0.35. REDOX fuel processed in 1964 was 1,693 metric tons of uranium (MTU) (Kupfer et al. 1995), so the fraction received by tank $241-\mathrm{U}-108$ is $0.35 \times 1,693 \mathrm{MTU}$ or $599.8 \mathrm{MTU}$. This waste contained about $47 \mathrm{~kg} \mathrm{Al} / \mathrm{MTU}, 0.47 \mathrm{~kg} \mathrm{Ni} / \mathrm{MTU}$ and $1.31 \mathrm{~kg} \mathrm{Si} / \mathrm{MTU}$. If the 1964 volume of REDOX cladding waste in 241-U-108, represented by the $599.8 \mathrm{MTU}$, was the main source of $\mathrm{Al}, \mathrm{Ni}$, and $\mathrm{Si}$, the inventories of these constituents would be $28,193 \mathrm{~kg}$ $\mathrm{Al}, 282 \mathrm{~kg} \mathrm{Ni}$, and $785 \mathrm{~kg} \mathrm{Si}$. The sample-based inventories for these elements are 51,190 $\mathrm{kg} \mathrm{Al}, 150 \mathrm{~kg} \mathrm{Ni}$, and $830 \mathrm{~kg} \mathrm{Si}$. This comparison suggests that the latter 1968 transfers of $2,840 \mathrm{~kL}$ ( $750 \mathrm{kgal}$ ) may have been supernatant that contributed additional $\mathrm{Al}$, but the earlier transfers in 1964 may have contributed much of the $\mathrm{Ni}$ and $\mathrm{Si}$.

\section{D3.2.3 Saltcake}

Over 90 percent of the waste in tank 241-U-108 is saltcake derived from REDOX cladding waste and 242-S evaporator bottoms. Of these saltcake wastes, cladding waste was the first type received, followed by evaporator bottoms. The HDW model separates saltcake into S1 and $\mathrm{S} 2$ categories based on feed source and process period with $\mathrm{S} 1$ assigned to the 242-S Evaporator campaign of 1973 to 1976 . For some of the major elements, such as aluminum and sulfate, the core sample analysis indicates a subtle increase in concentration in the core segments in approximately the lower tank half, suggesting these lower segments may be dominated by cladding waste.

In general, complete sample recovery was obtained from the lower segments and the composition of the segments, particularly segments $5,6,7$, and 8 , was similar from core to core for all three cores. These observations suggest that the waste volume represented by these segments is relatively homogeneous and may be representative of the earlier evaporator campaigns used for the SMMS1 HDW model. In addition, average sample-derived concentrations from TCRs for several other tanks containing wastes designated as SMMS1 saltcake have been prepared as part of the best-basis effort. This sample-based S1 saltcake concentration average is obtained from TCR's for tanks 241-S-101, 241-S-102, 241-U-106, and 241-U-109. These three data sets, the core segment data, the SMMS1 HDW model results for tank 241-U-108, and the average S1 saltcake tank data, were compared with 
inventories of $\mathrm{S} 1$ saltcake. This comparison is used to show how well tank 241-U-108 saltcake inventories compare with similar tanks.

Core segments $5,6,7$, and 8 from tank 241-U-108 were used, and only those saltcake components reported above detection limits were used. Table D3-3 compares concentrations of 241-U-108 analytes with the average SMMS1 composition from the HDW model and an average sample-based concentrations from TCRs for saltcake dominant tanks (241-S-101, $-\mathrm{S}-102$, $-\mathrm{U}-109$, and $-\mathrm{U}-106)$. The sample concentration data for tank 241-U-108 is an average of 12 core segment analyses from 3 cores for segments 5, 6, 7, and 8. Except for phosphate and fluoride, the U-108 concentrations are in general agreement with the average sample-based tank concentrations for S1 saltcake. Except for Al, there is general consistency between tank 241-U-108 concentrations and the SMMS1 HDW model results.

Table D3-3. Comparisons of Concentrations in Segments 5, 6, 7, 8 Sample Analysis Average With S1 Saltcake Type.

\begin{tabular}{|c|c|c|c|}
\hline Hnaturo & 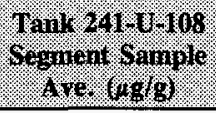 & 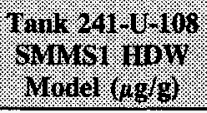 & 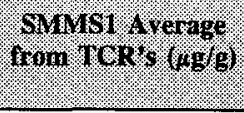 \\
\hline $\mathrm{Na}$ & $2.55 \mathrm{E}+05$ & $1.98 \mathrm{E}+05$ & $1.82 E+05$ \\
\hline $\mathrm{Al}$ & 16,489 & 32,089 & 15,083 \\
\hline $\mathrm{Cr}$ & 4,270 & 3,166 & 5,441 \\
\hline $\mathrm{PO}_{4}$ & 8,863 & 6,015 & 33,965 \\
\hline $\mathrm{SO}_{4}$ & 17,962 & 14,249 & 13,768 \\
\hline $\mathrm{F}$ & 1,043 & 806 & 6,255 \\
\hline $\mathrm{Cl}$ & 3,880 & 2,580 & 3,842 \\
\hline $\mathrm{NO}_{3}$ & $2.67 \mathrm{E}+05$ & $2.69 E+05$ & $1.63 E+05$ \\
\hline
\end{tabular}

The sample-based inventory of the components in Table D3-3 is based on the volume of tank 241-U-108 represented by the core segments. The sample-based inventory is compared to the HDW-based inventory of the tank 241-U-108 SMMS1 saltcake. A measured average density of $1.71 \mathrm{~kg} / \mathrm{L}$ was used for the sample segment data and an estimated density of $1.62 \mathrm{~kg} / \mathrm{L}$ was used for the SMMS1 HDW model data for tank 241-U-108.

The estimated inventories in Table D3-4 for the presumed S1 saltcake volume of tank 241-U-108 assumed to be dominated by REDOX cladding waste are somewhat higher, except for $\mathrm{Al}$, than those derived from SMMS1 HDW model results. Except for $\mathrm{Al}$, the inventories differ by less than a factor of 2 and are often within 30 percent. 
Table D3-4. Inventory Comparisons of Tank Volume Based on Tank 241-U-108 Selected Core Segment Analysis With HDW Model SMMS1 Saltcake Inventory.

\begin{tabular}{|c|c|c|c|c|}
\hline (.). & 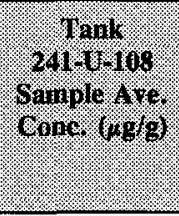 & 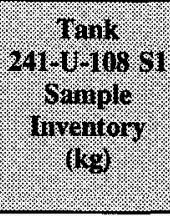 & 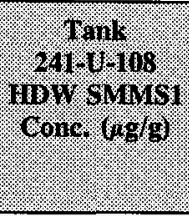 & 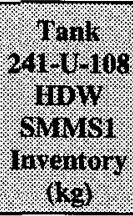 \\
\hline $\mathrm{Na}$ & 255,000 & 343,100 & 198,000 & 280,400 \\
\hline $\mathrm{Al}$ & 16,489 & 22,200 & 32,089 & 45,400 \\
\hline $\mathrm{Cr}$ & 4,270 & 5,740 & 3,166 & 4,480 \\
\hline $\mathrm{PO}_{4}$ & 8,863 & 11,900 & 6,015 & 8,520 \\
\hline $\mathrm{SO}_{4}$ & 17,962 & 24,200 & 14,249 & 20,200 \\
\hline $\mathrm{F}$ & 1,043 & 1,900 & 806 & 1,140 \\
\hline $\mathrm{Cl}$ & 3,880 & 5,200 & 2,580 & 3,650 \\
\hline $\mathrm{NO}_{3}$ & 267,000 & 359,300 & 269,000 & 381,000 \\
\hline Density, $\mathrm{kg} / \mathrm{L}$ & 1.71 & & 1.62 & \\
\hline Sample Mass, kg & $1.345 E+06$ & & $1.416 \mathrm{E}+06$ & \\
\hline
\end{tabular}

\section{D3.3 DOCUMENT ELEMENT BASIS}

Significant differences between sample-based and HDW inventories were apparent for Al, $\mathrm{Cr}$, phosphate and carbonate, which vary by a factor of two, and $\mathrm{Bi}, \mathrm{Ca}$, and $\mathrm{U}$ which differ by an order of magnitude. A discussion of the two inventory estimates for selected analytes is given below. Only those analytes present in core samples at concentrations greater than detection limits were considered.

\section{D3.3.1 Aluminum}

The sample-based and HDW inventories for aluminum are $51,190 \mathrm{~kg}$ and $96,800 \mathrm{~kg}$, respectively. The comparison of $\mathrm{S} 1$ saltcake type inventories suggests $22,200 \mathrm{~kg}$ and $45,400 \mathrm{~kg}$, respectively, which varies by a factor of two. However, Al concentrations of $16,500 \mu \mathrm{g} / \mathrm{g}$ from segments $5,6,7$, and 8 closely agree with the HDW saltcake average of $15,083 \mu \mathrm{g} / \mathrm{g}$. 


\section{D3.3.2 Bismuth}

The sample-based and HDW inventories for bismuth are $<5,930 \mathrm{~kg}$, (based on samples below detection limits) and $436 \mathrm{~kg}$, respectively. Because the Bi values for core samples were below detection limits and the detection limits were high, an alternate approach was used as a basis for a best $\mathrm{Bi}$ estimate. Average sample-based Bi concentrations for SMMS1 saltcake based on TCRs for tanks 241-S-101, -S-102, -U-106, and -U-109 yield a Bi concentration of $161 \mathrm{mg} / \mathrm{kg}$. The HDW model estimate for SMMS1 volume in tank 241-U-108 is $874 \mathrm{~kL}$ (Table D-6) and the average measured core sample density for tank $241-\mathrm{U}-108$ is $1.71 \mathrm{~kg} / \mathrm{L}$ (Table D-1). The product of these values yields an estimate of $241 \mathrm{~kg} \mathrm{Bi}$ for the $\mathrm{S} 1$ saltcake. For S2 saltcake, the average sample-derived Bi concentration from tanks 241-S-101, $-\mathrm{S}-102,-\mathrm{U}-102,-\mathrm{U}-107$, and $-\mathrm{U}-109$ is also $161 \mathrm{mg} / \mathrm{kg}$. Using the same measured density of $1.71 \mathrm{~kg} / \mathrm{L}$ and an HDW model SMMS2 volume of $696 \mathrm{~kL}$ (Table D-6) yields an S2 saltcake estimate of $192 \mathrm{~kg}$ for Bi. The sum of the S1 (241 kg) and $\mathrm{S} 2(192 \mathrm{~kg})$ saltcake estimate for Bi plus a measured liquid sample value of $4 \mathrm{~kg}$ yields a total $\mathrm{Bi}$ estimate for tank $241-\mathrm{U}-108$ of $437 \mathrm{~kg}$. Furthermore, process chemistry suggests that $\mathrm{Bi}$ concentrations will be low. Bismuth is not soluble, and thus will not be a substantial component of saltcake. Bismuth is absent from the REDOX process entirely.

\section{D3.3.3 Calcium}

The sample-based and HDW inventories for calcium are $<5,930$ (where all samples were below detection limits by the fusion method) and $3,940 \mathrm{~kg}$, respectively. An acid digest value of $447 \mathrm{~kg}$ from core composite data was well above detection limits and is considered the best estimate. The source of $\mathrm{Ca}$ in the HDW would appear to be due to the hard water and supernatant mixing assumptions made for the SMMS1 and/or SMMS2 models.

\section{D3.3.4 Chromium}

The sample-based and HDW inventories for chromium are $12,116 \mathrm{~kg}$ and $5,980 \mathrm{~kg}$, respectively. The comparison of S1 saltcake types based on sample data and HDW data suggest partial inventories of $5,740 \mathrm{~kg}$ and $4,480 \mathrm{~kg}$, respectively. The HDW model indicates that $5,980 \mathrm{~kg}$ of chromium is introduced to tank 241-U-108 in the SMMS1 and SMMS2 models. The solubility assumptions in these models, especially regarding REDOX waste, have not been verified.

\section{D3.3.5 Sulfate}

The sample-based and HDW inventories for sulfate are $44,430 \mathrm{~kg}$ and $45,000 \mathrm{~kg}$, respectively. Estimates for the $\mathrm{S} 1$ saltcake portion are $24,200 \mathrm{~kg}$ for sample-based inventory and $20,200 \mathrm{~kg}$ from HDW data. The HDW model indicates that $45,000 \mathrm{~kg}$ of sulfate are introduced to tank 241-U-108 in the SMMS1 and SMMS2 models. This number is consistent 
with the sample-based inventory, indicating that model assumptions about sulfate are reasonable. However, good sample recovery and consistency among cores taken from 241-U-108 suggest sample-based inventories are more reliable.

\section{D3.3.6 Phosphate}

The sample-based and HDW inventories for phosphate are $50,527 \mathrm{~kg}$ and $16,800 \mathrm{~kg}$, respectively. Estimates for the $S 1$ saltcake portion are $11,900 \mathrm{~kg}$ from sample data and $8,520 \mathrm{~kg}$ from HDW data, respectively. Comparison of these values suggests that the HDW model estimates are too low because the phosphate solubility assumed is too high for this waste.

\section{D4.0 ESTABLISH THE BEST BASIS AND ESTABLISH COMPONENT INVENTORIES}

The results from this evaluation support using the sampling data for tank 241-U-108 for the following reasons.

1. Core sample data were available from three risers at three widely spaced positions. Recovery of sample segments was good for most samples and consistent from core to core. Also, concentrations in each segment were consistent from core to core. Upon inspection of data collection and analysis protocols, no reasons were found to reject the laboratory data.

2. The sample-based inventory reconciles better with the position that the sludge layer in the tank is REDOX CW rather than bismuth phosphate MW and that sluicing of earlier metal waste was complete.

3. The evaporator concentrate waste (SMMS1 and SMMS2) that make up the majority of the waste volume in tank 241-U-108 have no independent data source with which to they may be compared. The process of mixing and evaporating supernatants is sufficiently complex that comparison to process flowsheets or multicomponent chemical modeling is impractical.

Best-basis inventory estimates for tank 241-U-108 are presented in Tables D4-1 and D4-2. The sample-based inventory values were generated using a measured density of $1.74 \mathrm{~g} / \mathrm{mL}$ for segment sample data, $1.40 \mathrm{~g} / \mathrm{mL}$ for drainable liquid samples, and $1.71 \mathrm{~g} / \mathrm{mL}$ for core composites. 
Table D4-1. Best-Basis Inventory Estimates for Nonradioactive Components in Tank 241-U-108. (2 sheets)

\begin{tabular}{|c|c|c|c|}
\hline 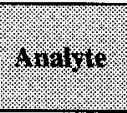 & 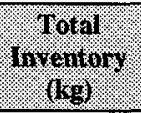 & $(\mathrm{s}, \mathrm{g} / \mathrm{g}, \mathrm{g}, \mathrm{g})$ & 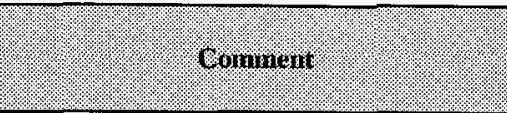 \\
\hline $\mathrm{Al}$ & 51,190 & $\mathbf{S}$ & $\begin{array}{l}\text { Average segment sample data plus liquid } \\
\text { data }\end{array}$ \\
\hline $\mathrm{Bi}$ & 437 & $\mathrm{E}$ & $\begin{array}{l}\text { Average based on S1 and S2 TCR saltcake } \\
\text { data }\end{array}$ \\
\hline $\mathrm{Ca}$ & 447 & $S$ & $\begin{array}{l}\text { Average based on acid digest of core } \\
\text { composite data }\end{array}$ \\
\hline $\mathrm{Cl}$ & 10,914 & $\mathrm{~S}$ & $\begin{array}{l}\text { Average segment sample data plus liquid } \\
\text { data }\end{array}$ \\
\hline $\begin{array}{c}\mathrm{TIC} \text { as } \\
\mathrm{CO}_{3}\end{array}$ & 118,500 & $S$ & $\begin{array}{l}\text { Average segment sample data plus liquid } \\
\text { data }\end{array}$ \\
\hline $\mathrm{Cr}$ & 12,116 & $S$ & $\begin{array}{l}\text { Average segment sample data plus liquid } \\
\text { data }\end{array}$ \\
\hline $\mathrm{F}$ & 2,836 & $S$ & $\begin{array}{l}\text { Average segment sample data plus liquid } \\
\text { data }\end{array}$ \\
\hline $\mathrm{Fe}$ & 507 & $S$ & Core composite acid digest samples \\
\hline $\mathrm{Hg}$ & $\mathrm{n} / \mathrm{r}$ & & \\
\hline $\mathrm{K}$ & 4,800 & $S$ & Core composite samples \\
\hline La & $<117$ & $S$ & $\begin{array}{l}\text { Core composite samples below detection } \\
\text { limit }\end{array}$ \\
\hline $\mathrm{Mn}$ & 185 & $S$ & Core composite acid digest samples \\
\hline $\mathrm{Na}$ & 726,100 & $S$ & $\begin{array}{l}\text { Average segment sample data plus liquid } \\
\text { data }\end{array}$ \\
\hline $\mathrm{Ni}$ & 150 & $S$ & Core composite acid digest samples \\
\hline $\mathrm{NO}_{2}$ & 157,800 & $S$ & $\begin{array}{l}\text { Average segment sample data plus liquid } \\
\text { data }\end{array}$ \\
\hline $\mathrm{NO}_{3}$ & 879,500 & $\mathbf{S}$ & $\begin{array}{l}\text { Average segment sample data plus liquid } \\
\text { data }\end{array}$ \\
\hline $\mathrm{OH}$ & $\mathrm{n} / \mathrm{r}$ & & \\
\hline $\mathrm{Pb}$ & $<234$ & $\mathrm{~S}$ & $\begin{array}{l}\text { Core composite samples below detection } \\
\text { limit }\end{array}$ \\
\hline $\mathrm{P}$ as $\mathrm{PO}_{4}$ & 50,527 & $S$ & $\begin{array}{l}\text { Average segment sample data plus liquid } \\
\text { data }\end{array}$ \\
\hline
\end{tabular}


Table D4-1. Best-Basis Inventory Estimates for Nonradioactive Components in Tank 241-U-108. (2 sheets)

\begin{tabular}{|c|c|c|c|}
\hline 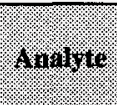 & 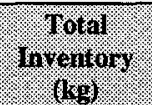 & $(8,4)$, or $n$ & Coininient. \\
\hline $\mathrm{Si}$ & 830 & $\mathbf{S}$ & $\begin{array}{l}\text { Average core composite water wash sample } \\
\text { plus liquid }\end{array}$ \\
\hline $\mathrm{S}$ as $\mathrm{SO}_{4}$ & 44,430 & $\mathrm{~S}$ & $\begin{array}{l}\text { Average segment sample data plus liquid } \\
\text { data }\end{array}$ \\
\hline $\mathrm{Sr}$ & $<23.4$ & $S$ & Core composite below detection limit \\
\hline TOC & 12,850 & $S$ & $\begin{array}{l}\text { Average segment sample data plus liquid } \\
\text { data }\end{array}$ \\
\hline $\mathrm{U}_{\text {TOTAL }}$ & 538 & $S$ & $\begin{array}{l}\text { Laser phosphorescence data from core } \\
\text { composite samples }\end{array}$ \\
\hline $\mathrm{Zr}$ & $<23.4$ & $S$ & Core composite below detection limit \\
\hline
\end{tabular}

Notes:

${ }^{1} S$ = Sample-based (see Appendix B)

$\mathbf{M}=$ Hanford Defined Waste model-based

$\mathrm{E}=$ Engineering assessment-based

Table D4-2. Best-Basis Inventory Estimates for Radioactive Components in Tank 241-U-108. (3 sheets)

\begin{tabular}{|c|c|c|c|}
\hline 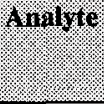 & $\begin{array}{l}\text { lotal } \\
\text { inventoms. }\end{array}$ & 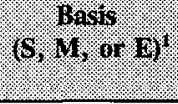 & Commerat \\
\hline${ }^{3} \mathrm{H}$ & $\mathrm{n} / \mathrm{r}$ & & \\
\hline${ }^{14} \mathrm{C}$ & $\mathrm{n} / \mathrm{r}$ & & \\
\hline${ }^{59} \mathrm{Ni}$ & $\mathrm{n} / \mathrm{r}$ & & \\
\hline${ }^{60} \mathrm{Co}$ & $<61.5$ & $S$ & Average solid segment data \\
\hline${ }^{63} \mathrm{Ni}$ & $\mathrm{n} / \mathrm{r}$ & & \\
\hline${ }^{79} \mathrm{Se}$ & $\mathrm{n} / \mathrm{r}$ & & \\
\hline${ }^{90} \mathrm{Sr}$ & 29,400 & $S$ & Average solid segment data \\
\hline${ }^{90} \mathrm{Y}$ & $\mathrm{n} / \mathrm{r}$ & & \\
\hline${ }^{93} \mathrm{Zr}$ & $\mathrm{n} / \mathrm{r}$ & & \\
\hline${ }^{93 \mathrm{~m}} \mathrm{Nb}$ & $n / r$ & & \\
\hline${ }^{99} \mathrm{Tc}$ & $\mathrm{n} / \mathrm{r}$ & & \\
\hline
\end{tabular}


HNF-SD-WM-ER-639 Rev. 0

Table D4-2. Best-Basis Inventory Estimates for Radioactive Components in Tank 241-U-108. (3 sheets)

\begin{tabular}{|c|c|c|c|}
\hline \%ongro & 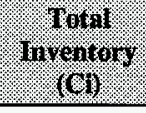 & 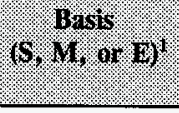 & 6ominent \\
\hline${ }^{106} \mathrm{Ru}$ & $\mathrm{n} / \mathrm{r}$ & & \\
\hline${ }^{113 \mathrm{~m}} \mathrm{Cd}$ & $\mathrm{n} / \mathrm{r}$ & & \\
\hline${ }^{125} \mathrm{Sb}$ & $\mathrm{n} / \mathrm{r}$ & & \\
\hline${ }^{126} \mathrm{Sn}$ & $\mathrm{n} / \mathrm{r}$ & & \\
\hline${ }^{129} \mathrm{I}$ & $\mathrm{n} / \mathrm{r}$ & & \\
\hline${ }^{134} \mathrm{Cs}$ & $\mathrm{n} / \mathrm{r}$ & & \\
\hline${ }^{137} \mathrm{Cs}$ & 411,000 & $S$ & Average core segment data \\
\hline${ }^{137 \mathrm{~m}} \mathrm{Ba}$ & $\mathrm{n} / \mathrm{r}$ & & \\
\hline${ }^{151} \mathrm{Sm}$ & $\mathrm{n} / \mathrm{r}$ & & \\
\hline${ }^{152} \mathrm{Eu}$ & $\mathrm{n} / \mathrm{r}$ & & \\
\hline${ }^{154} \mathrm{Eu}$ & $<241$ & $S$ & Average core segment data \\
\hline${ }^{155} \mathrm{Eu}$ & $<884$ & $S$ & Average core segment data \\
\hline${ }^{226} \mathrm{Ra}$ & $\mathrm{n} / \mathrm{r}$ & & \\
\hline${ }^{227} \mathrm{Ac}$ & $\mathrm{n} / \mathrm{r}$ & & \\
\hline${ }^{228} \mathrm{Ra}$ & $\mathrm{n} / \mathrm{r}$ & & \\
\hline${ }^{229} \mathrm{Th}$ & $\mathrm{n} / \mathrm{r}$ & & \\
\hline${ }^{231} \mathrm{~Pa}$ & $\mathrm{n} / \mathrm{r}$ & & \\
\hline${ }^{232} \mathrm{Th}$ & $\mathrm{n} / \mathrm{r}$ & & \\
\hline${ }^{232} \mathrm{U}$ & $\mathrm{n} / \mathrm{r}$ & & \\
\hline${ }^{233} \mathrm{U}$ & $\mathrm{n} / \mathrm{r}$ & & \\
\hline${ }^{234} \mathrm{U}$ & $\mathrm{n} / \mathrm{r}$ & & \\
\hline${ }^{235} \mathrm{U}$ & $\mathrm{n} / \mathrm{r}$ & & \\
\hline${ }^{236} \mathrm{U}$ & $\mathrm{n} / \mathrm{r}$ & & \\
\hline${ }^{237} \mathrm{~Np}$ & $\mathrm{n} / \mathrm{r}$ & & \\
\hline${ }^{238} \mathrm{Pu}$ & $n / r$ & & \\
\hline${ }^{238} \mathrm{U}$ & $\mathrm{n} / \mathrm{r}$ & & \\
\hline${ }^{239} \mathrm{Pu}$ & $\mathrm{n} / \mathrm{r}$ & & \\
\hline
\end{tabular}


Table D4-2. Best-Basis Inventory Estimates for Radioactive Components in Tank 241-U-108. (3 sheets)

\begin{tabular}{|c|c|c|c|}
\hline 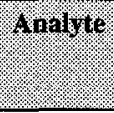 & 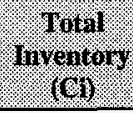 & 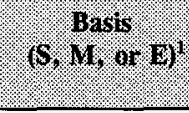 & 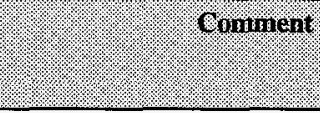 \\
\hline${ }^{240} \mathrm{Pu}$ & $\mathrm{n} / \mathrm{r}$ & & \\
\hline${ }^{241} \mathrm{Am}$ & $<1,930$ & $S$ & Average core segment data \\
\hline${ }^{24 I} \mathrm{Pu}$ & $\mathrm{n} / \mathrm{r}$ & & \\
\hline${ }^{242} \mathrm{Cm}$ & $\mathrm{n} / \mathrm{r}$ & & \\
\hline${ }^{242} \mathrm{Pu}$ & $\mathrm{n} / \mathrm{r}$ & & \\
\hline${ }^{243} \mathrm{Am}$ & $\mathrm{n} / \mathrm{r}$ & & \\
\hline${ }^{243} \mathrm{Cm}$ & $\mathrm{n} / \mathrm{r}$ & & \\
\hline${ }^{244} \mathrm{Cm}$ & $\mathrm{n} / \mathrm{r}$ & & \\
\hline
\end{tabular}

Notes:

$$
\begin{aligned}
& { }^{1} \mathrm{~S}=\text { Sample-based } \\
& \mathrm{M}=\text { Hanford Defined Waste model-based } \\
& \mathrm{E}=\text { Engineering assessment-based }
\end{aligned}
$$




\section{D5.0 APPENDIX D REFERENCES}

Agnew, S. F., J. Boyer, R. Corbin, T. Duran, J. FitzPatrick, K. Jurgensen, T. Ortiz, and B. Young, 1996a, Hanford Tank Chemical and Radionuclide Inventories: HDW Model Rev. 3, 1996, Los Alamos National Laboratory, Los Alamos, New Mexico.

Agnew, S. F., R. A. Corbin, T. B. Duran, K. A. Jurgensen, T. P. Ortiz, and B. L. Young, 1996b, Waste Status and Transaction Record Summary for the Southwest Quadrant of the Hanford 200 East Area, WHC-SD-WM-TI-614, Rev. 2, Los Alamos National Laboratory, Los Alamos, New Mexico.

Anderson, J. D., 1990, A History of the 200 Area Farms, WHC-MR-0132, Westinghouse Hanford Company, Richland, Washington.

Bell, K. E., 1996, Final Report for Tank 241-U-108, Push-Mode Core Samples 141, 145, and 146 Analytical Results for the Final Report, WHC-SD-WM-DP-198, Rev. 0, Westinghouse Hanford Company, Richland, Washington.

Hill, J. G., G. S. Anderson, and B. C. Simpson, 1995, The Sort on Radioactive Waste Type Model: A Method to Sort Single-Shell Tanks into Characteristic Groups, PNL-9814, Rev. 2, Pacific Northwest Laboratory, Richland, Washington.

Kupfer, M. J., M. D. LeClair, W. W. Schulz, and L. W. Shelton, 1995, Work Plan for Defining a Standard Inventory Estimate for Wastes Stored in Hanford Site Underground Tanks, WHC-SD-WM-WP-311, Rev. 0, Westinghouse Hanford Company, Richland, Washington.

Rodenhizer, D. G., 1987, Hanford Waste Tank Sluicing History, WHC-SD-WM-TI-302, Rev. 0, Westinghouse Hanford Company, Richland, Washington. 
HNF-SD-WM-ER-639 Rev. 0

APPENDIX E

BIBLIOGRAPHY FOR TANK 241-U-108 
HNF-SD-WM-ER-639 Rev. 0

This page left blank intentionally. 


\section{APPENDIX E}

\section{BIBLIOGRAPHY FOR TANK 241-U-108}

Appendix E provides a bibliography of information that supports the characterization of tank 241-U-108. This bibliography represents an in-depth literature search of all known information sources that provide sampling, analysis, surveillance, and modeling information, as well as processing occurrences associated with tank $241-\mathrm{U}-108$ and its respective waste types.

The references in this bibliography are separated into three broad categories containing references broken down into subgroups. These categories and their subgroups are listed below.

\section{NON-ANALYTICAL DATA}

Ia. Models/Waste Type Inventories/Campaign Information

Ib. Fill History/Waste Transfer Records

Ic. Surveillance/Tank Configuration

Id. Sample Planning/Tank Prioritization

Ie. Data Quality Objectives/Customers of Characterization Data

\section{ANALYTICAL DATA - SAMPLING OF TANK WASTE AND WASTE TYPES}

IIa. Sampling of tank 241-U-108

IIb. Sampling of 242-S Evaporator Waste Streams

\section{COMBINED ANALYTICAL/NON-ANALYTICAL DATA}

IIIa. Inventories using both Campaign and Analytical Information

IIIb. Compendium of Existing Physical and Chemical Documented Data Sources

This bibliography is broken down into the appropriate sections of material to use, with an annotation at the end of each reference describing the information source. Where possible, a reference is provided for information sources. A majority of the information listed below may be found in the Lockheed Martin Hanford Corporation Tank Characterization Resource Center. 


\section{Non-Analytical Data}

Ia. Models/Waste Type Inventories/Campaign Information

Agnew, S. F., J. Boyer, R. Corbin, T. Duran, J. FitzPatrick, K. Jurgensen, T. Ortiz, and B. Young, 1996, Hanford Tank Chemical and Radionuclide Inventories: HDW Model Rev. 3, LA-UR-96-858, Los Alamos National Laboratory, Los Alamos, New Mexico.

- Document contains tank layer and supernatant mixing models and the historical tank content estimate for Hanford Site 200 East and 200 West underground waste storage tanks, as well as a list of Hanford Site waste types and their respective major analyte concentrations.

Anderson, J.D., 1990, History of the 200 Area Tank Farms, WHC-MR-0132, Westinghouse Hanford Company, Richland, Washington.

- Document contains single-shell tank fill history and primary campaign/waste type information up to 1981.

Boldt, A. L., 1966, REDOX Chemical Flowsheet HW No. 9, ISO-335, Isochem, Inc., Richland, Washington.

- Document contains compositions of material balance for REDOX process as well as a separations plan denoting process stream waste before transfer to 200 Area waste tanks.

Crawley, D. T., 1960, REDOX Chemical Flowsheet HW-No. 6, HW-66203, Hanford Atomic Products Operation, General Electric Company, Richland, Washington.

- Document contains compositions of material balance for REDOX process as well as a separations plan denoting process stream waste before transfer to 200 Area waste tanks.

Jungfleisch, F.M., B.C. Simpson, 1993, Preliminary Estimation of the Waste Inventories in Hanford Tanks Through 1980, SD-WM-TI-057, Rev. 0A, Westinghouse Hanford Company, Richland, Washington.

- A model based on process knowledge and radioactive decay estimations using ORIGEN for different compositions of process waste streams assembled for total, solution, and solids compositions per tank. Assumptions about waste/waste types and solubility parameters/constraints are also given. 
Merrill, E. T., and R. L. Stevenson, 1955, REDOX Chemical Flowsheet $H W$ No. 5, HW-38684, Hanford Atomic Products Operation, Richland, Washington.

- Document contains compositions of material balance for REDOX process as well as a separations plan denoting process stream waste before transfer to 200 Area waste tanks.

Schneider, K.J., 1951, Flowsheets and Flow Diagrams of Precipitation Separations Process, HW-23043, Hanford Atomic Products Operation, Richland, Washington.

- Document contains compositions of process stream waste before transfer to 200 Area waste tanks.

Ib. Fill History/Waste Transfer Records

Agnew, S.F., R. A. Corbin, T. B. Duran, K. A. Jurgensen, T. P. Ortiz, and B. L. Young, 1996, Waste Status and Transaction Record Summary for the Southwest Quadrant, WHC-SD-WM-TI-614, Rev. 1, Westinghouse Hanford Company, Richland, Washington.

- Document contains spreadsheets depicting all available data on tank additions/transfers for SW quadrant.

Anderson, J.D., 1990, A History of the 200 Area Tank Farms, WHC-MR-0132, Westinghouse Hanford Company, Richland, Washington.

- Document contains single-shell tank fill history and primary campaign/waste type information up to 1981 .

Ic. Surveillance/Tank Configuration

Alstad, A. T., 1993, Riser Configuration Document for Single-Shell Waste Tanks, WHC-SD-RE-TI-053, Rev. 9, Westinghouse Hanford Company, Richland, Washington.

- Document shows tank riser locations in relation to tank aerial view as well as a description of riser and its contents.

Bergmann, L. M., 1991, Single-Shell Tank Isolation Safety Analysis Report, WHC-SD-WM-SAR-006, Rev. 2, Westinghouse Hanford Company, Richland, Washington.

- Document contains safety analysis report on isolation of single-shell tanks. 
Lipnicki, J., 1996, Waste Tank Risers Available for Sampling, WHC-SD-WM-TI-710, Rev. 3, Westinghouse Hanford Company, Richland, Washington.

- Document gives an assessment of all risers per tank, however not all tanks are included/completed.

Rogers, R. D., and H. T. Daniels, 1944, Specifications for Construction of Composite Storage Tanks Bldg. \#241 at Hanford Engineer Works, CVI-73550, E. I. Du Pont de Nemours \& Co., Richland, Washington.

- Vendor information contains a compilation of drawings for Hanford 200 East and 200 West Area tanks.

Swaney, S. L., 1994, Single-Shell Stabilization Record, WHC-SD-RE-TI-178, Rev. 4, Westinghouse Hanford Company, Richland, Washington.

- Document contains information about the stabilization of single-shell tanks.

Tran, T. T., 1993, Thermocouple Status Single Shell \& Double Shell Waste Tanks, WHC-SD-WM-TI-553, Rev. 0, Westinghouse Hanford Company, Richland, Washington.

- Document contains thermocouple data for each tank including some history and riser information.

Vitro Engineering Corporation, 1988, Piping Waste Tank Isolation TK 241-U-108, H-2-73156, Rev. 4, Vitro Engineering Corporation, Richland, Washington.

- Drawing shows riser layout for tank 241-U-108.

Id. Sample Planning/Tank Prioritization

Brown, T. M., J. W. Hunt, and T. J. Kunthara, 1996, Tank Waste Characterization Basis, WHC-SD-WM-TA-164, Rev. 2, Westinghouse Hanford Company, Richland, Washington.

- Document that summarizes the technical basis for characterizing the waste in the tanks and assigns a priority number to each tank. 
Christensen, W. R., 1976, Tank Farm Sludge Samples, (internal memorandum to J. A. Teal, February 19), Atlantic Richfield Hanford Company, Richland, Washington.

- Memorandum requests salt samples from tank 241-U-108.

Grimes, G. W., 1977, Hanford Long-Term Defense High-Level Waste Management Program Waste Sampling and Characterization Plan, RHO-CD-137, Rockwell Hanford Operations, Richland, Washington.

- Document contains plan for characterizing waste, short and long term goals, tank priority, analysis needs, estimates of analyte concentrations per waste type, and a characterization flowsheet.

Homi, C. S., 1996, Tank 241-U-108 Push Mode Core Sampling and Analysis Plan, WHC-SD-WM-TSAP-049, Rev. 0, Westinghouse Hanford Company, Richland, Washington.

- Document contains detailed sampling and analysis scheme for core samples to be taken from tank 241-U-108 to address applicable DQOs.

Homi, C. S., 1995, Vapor Sampling and Analysis Plan, WHC-SD-WM-TP-335, Rev. OF, Westinghouse Hanford Company, Richland, Washington.

- Document contains vapor sampling and analysis scheme for tank 241-U-108.

Osborne, J. W., and J. L. Huckaby, 1994, Program Plan for the Resolution of Tank Vapor Issues, WHC-EP-0562, Rev. 1, Westinghouse Hanford Company, Richland, Washington.

- Document presents plan/approach to resolving Hanford Site waste tank vapor concerns.

Puryear, D. A., 1971, Waste Tank Characterization, (internal memorandum to D. A. Dodd, August 2), Atlantic Richfield Hanford Company, Richland, Washington.

- Memorandum contains analytes desired from tank sampled in support of 242-S Evaporator startup. 
Schreiber, R. D., 1995, Compatibility Grab Sampling and Analysis Plan, WHC-SD-WM-TP-330, Rev. 0, Westinghouse Hanford Company, Richland, Washington.

- Document contains compatibility sampling needs for tank 241-U-108.

Walser, R. L., 1975, Sludge Samples Required From July 21 to December 31, 1975, (internal memorandum to J. A. Teal, July 17), Atlantic Richfield Hanford Company, Richland, Washington.

- Memorandum requests sludge samples from tank 241-U-108.

Westinghouse Hanford Company, 1996, Tank U-108 Core Sampling Work Package, WS-95-00250, Westinghouse Hanford Company, Richland, Washington.

- Document contains sampling procedures for tank 241-U-108.

Winkelman, W. D., 1996, Tank 241-U-108 Tank Characterization Plan, WHC-SD-WM-TP-315, Rev. 3, Westinghouse Hanford Company, Richland, Washington.

- Document discusses all relevant DQOs and how their requirements will be met for tank 241-U-108.

Winkelman, W. D., J. W. Hunt, and L. J. Fergestrom, 1996, Fiscal Year 1997 Tank Waste Analysis Plan, WHC-SD-WM-PLN-120, Rev. 1, Lockheed Martin Hanford Company, Richland, Washington.

- Document contains Tri-Party Agreement requirement-driven TWRS Characterization program information and a list of tanks addressed in fiscal year 1997.

Ie. Data Quality Objectives/Customers of Characterization Data

Dukelow, G. T., J. W. Hunt, H. Babad, and J. E. Meacham., 1995, Tank Safety Screening Data Quality Objective, WHC-SD-WM-SP-004, Rev. 2, Westinghouse Hanford Company, Richland, Washington.

- Document contains objectives to sample all tanks for safety concerns (ferrocyanide, organic, flammable gas, and criticality) as well as decision thresholds for energetics, criticality and flammability. 
Duncan, J. B., D. W. Hendrickson, and R. K. Biyani, 1996, Hanford Single Shell Tank Saltcake Cesium Removal Test Plan, WHC-SD-RE-TP-024, Rev. 0, Westinghouse Hanford Company, Richland, Washington.

- Document provides test preparation and conduct of cesium removal test using single-shell tank saltcake, including tank 241-U-108.

Fowler, K. D.,1995, Data Quality Objectives for Tank Farms Waste Compatibility Program, WHC-SD-WM-DQO-001, Rev. 1, Westinghouse Hanford Company, Richland, Washington.

- Document contains waste transfer compatibility program data needs, list of tanks to be evaluated, decision thresholds, and decision logic flow diagram.

Hodgson, K. M. and M. D. LeClair, 1996, Work Plan for Defining a Standard Inventory Estimate for Wastes Stored in Hanford Site Underground Tanks, WHC-SD-WM-WP-311, Rev. 1, Lockheed Martin Hanford Company, Richland, Washington.

- Work plan contains task scope, deliverables, budget, and schedule for global and tank specific standard inventory estimates as well as information on change control and incorporation of standard inventories into the Tank Characterization Database.

Osborne, J. W., J. L. Huckaby, T. P. Rudolph, E. R. Hewitt, D. D. Mahlum, J. Y. Young, and C. M. Anderson, 1995, Data Quality Objectives for Generic In-Tank Health and Safety Issue Resolution, WHC-SD-WM-DQO-002, Rev. 1, Westinghouse Hanford Company, Richland, Washington.

- DQO used to determine if tank headspaces contain potentially flammable levels of gases and vapors and or if there is a potential for worker hazards associated with the toxicity of constituents in any vapor emissions from the tanks.

Simpson, B.C. and D. J. McCain, 1996, Historical Model Evaluation Data Requirements, WHC-SD-WM-DQO-018, Rev. 1, Westinghouse Hanford Company, Richland, Washington.

- Document identifies analytical parameters to characterize waste into one of five waste types. 
Turner, D.A., H. Babad, L. L. Buckley, J. E. Meacham, 1996, Data Quality Objective to Support Resolution of the Organic Complexant Safety Issue, WHC-SD-WM-DQO-006, Rev. 2, Westinghouse Hanford Company, Richland, Washington.

- Document contains organic program data needs, list of tanks to be evaluated, decision thresholds, and decision logic flow diagram.

\section{Analytical Data}

IIa. Sampling of tank 241-U-108

Bell, K. E., 1996, Final Report for Tank 241-U-108, Push-Mode Core Samples 141, 145, and 146, WHC-SD-WM-DP-198, Rev. 0, Westinghouse Hanford Company, Richland, Washington.

- Document contains analytical results from March-May 1996 push mode core sampling event.

Bell, K. E., 1996, Safety Screening/Immediate Notification for Tank 241-U-108, (internal memorandum \#79400-96-161 to J. N. Appel, H. Babad, D. C. Hetzer, J. E. Hyatt, T. J. Kelley, N. W. Kirch, M. J. Kupfer, J. E. Meacham, K. L. Powell, J. B. Schaffer, L. W. Shelton, and J. A. Voogd, August 8), Westinghouse Hanford Company, Richland, Washington.

- Memorandum contains notification of any analytical results exceeding DQO limits from March-May 1996 push mode core sampling event.

Caprio, G. S., 1995, Vapor and Gas Sampling of Single-Shell Tank 241-U-108 Using the Vapor Sampling System, WHC-SD-WM-RPT-181, Rev. 0, Westinghouse Hanford Company, Richland, Washington.

- Document presents sampling data from August 1995 vapor sampling event.

Esch, R. A., 1995, Waste Compatibility Results for 241-U-108 Grab Samples, (internal memorandum $\# 75970-95-043$ Rev. 1 to M. J. Sutey, R. D. Schreiber, S. D. Estey, and N. W. Kirch, July 24), Westinghouse Hanford Company, Richland, Washington.

- Document contains analytical results from May 1995 grab sampling event. 
Geier, R. G., 1976, Estimated Hanford Liquid Wastes Chemical Inventory as of June 30, 1976, ARH-CD-768, Atlantic Richfield Hanford Company, Richland, Washington.

- Document contains historical sample analytical results for tank 241-U-108 as well as many other tanks.

Horton, J. E., 1975, Analyses of Sludge Sample From Tank 108-U, (internal memorandum to W. R. Christensen, September 29), Atlantic Richfield Hanford Company, Richland, Washington.

- Memorandum contains historical sample analytical results for tank 241-U-108.

Horton, J. E., 1975, Concentration - Laboratory Assistance, (internal memorandum to D. C. Lini, October [day unknown]), Atlantic Richfield Hanford Company, Richland, Washington.

- Memorandum contains historical sample analytical results for tank 241-U-108.

Mahon, R. D., 1995, Vapor and Gas Sampling of Single-Shell Tank 241-U-108 Using the Vapor Sampling System, WHC-SD-WM-RPT-180, Rev. 0, Westinghouse Hanford Company, Richland, Washington.

- Document contains analytical results from August 1995 vapor sampling event.

Puryear D. A., 1971, Characterization of $S, U$, and SX Waste Tanks, (internal memorandum to J. O. Skolrud, September 21), Atlantic Richfield Hanford Company, Richland, Washington.

- Memorandum contains historical sample analytical results for tank 241-U-108.

Rice, A. D., 1996, Correction of Spike Recovery for Persulfate TIC/TOC Analyses on Tank Samples, (external letter \#9655794 to K. M. Hall, Lockheed Martin Hanford Company, November 20), Rust Federal Services of Hanford, Inc., Richland, Washington.

- Memorandum contains corrections to analytical results from March-May 1996 push mode core sampling event. 
Sant W. H., 1974, 242-S Feed Samples, Number T-151, Sample Point 108-U, (internal memorandum to R. L. Walker, January 21), Atlantic Richfield Hanford Company, Richland, Washington.

- Memorandum contains historical sample analytical results for tank 241-U-108.

Thomas, B. L., T. W. Clauss, J. C. Evana, B. D. McVeety, K. H. Pool, K. B. Olsen, J. S. Fruchter, and M. W. Ligotke, 1996, Headspace Vapor Characterization of Hanford Waste Tank 241-U-108: Results from Samples Collected on 08/29/95, PNNL-10961, Pacific Northwest National Laboratory, Richland, Washington.

- Document contains analytical results from August 1995 vapor sampling event.

Wheeler, R. E., 1976, Analysis of Tank Farm Samples, Sample: T-376, Tank: 108-U, Received: December 12, 1975, (internal memorandum to R. L. Walker, February 2), Atlantic Richfield Hanford Company, Richland, Washington.

- Memorandum contains historical sample analytical results for tank 241-U-108.

Wheeler, R. E., 1975, Analysis of Tank Farm Samples, Sample: T-6142, Tank: 108-U, Received: July 15, 1975, (internal memorandum to R. L. Walker, October 21), Atlantic Richfield Hanford Company, Richland, Washington.

- Memorandum contains historical sample analytical results for tank 241-U-108.

Wheeler, R. E., 1975, Analysis of Tank Farm Samples, Sample: T-6352, Tank: 108-U, Received: July 22, 1975, (internal memorandum to R. L. Walker, October 24), Atlantic Richfield Hanford Company, Richland, Washington.

- Memorandum contains historical sample analytical results for tank 241-U-108. 
Wheeler, R. E., 1975, Analysis of Tank Farm Samples, Sample: T-6709, Tank: 108-U, Received: August 4, 1975, (internal memorandum to R. L. Walker, October 21), Atlantic Richfield Hanford Company, Richland, Washington.

- Memorandum contains historical sample analytical results for tank 241-U-108.

Wheeler, R. E., 1975, Analysis of Tank Farm Samples, Sample: T-7019, Tank: 108-U, Received: August 12, 1975, (internal memorandum to R. L. Walker, October 21), Atlantic Richfield Hanford Company, Richland, Washington.

- Memorandum contains historical sample analytical results for tank 241-U-108.

Wheeler, R. E., 1975, Analysis of Tank Farm Samples, Sample: T-7443, Tank: 108-U, Received: August 26, 1975, (internal memorandum to R. L. Walker, December 2), Atlantic Richfield Hanford Company, Richland, Washington.

- Memorandum contains historical sample analytical results for tank 241-U-108.

Wheeler, R. E., 1975, Analysis of Tank Farm Samples, Sample: T-7822, Tank: 108-U, Received: September 8, 1975, (internal memorandum to R. L. Walker, October 20), Atlantic Richfield Hanford Company, Richland, Washington.

- Memorandum contains historical sample analytical results for tank 241-U-108.

IIb. Sampling of 242-S Evaporator Waste Streams

- The following analyses may provide insight as to the composition of saltcake waste type expected to be in tank 241-U-108.

Brown, G. E., 1978, Operating Parameters for Evaporator Crystallizers, (internal memorandum to K. G. Carothers, July 5), Rockwell Hanford Operations, Richland, Washington.

Campbell, G. D., 1975, 242-S Evaporator-Crystallizer Material Balance, (internal memorandum to R. L. Walker, August 5), Atlantic Richfield Hanford Company Operations, Richland, Washington. 
Puryear, D. A. and J. S. Buckingham, 1971, Status Report on Waste Solidification Studies and Separations Chemistry Laboratory, (internal memorandum to M. H. Campbell and Distribution, Process Aids \#00362, July 23), Atlantic Richfield Hanford Company Operations, Richland, Washington.

\section{Combined Analytical/Non-Analytical Data}

IIIa. Inventories from Campaign and Analytical Information

Agnew, S. F., J. Boyer, R. Corbin, T. Duran, J. FitzPatrick, K. Jurgensen, T. Ortiz, B. Young, 1996, Hanford Tank Chemical and Radionuclide Inventories: HDW Model Rev. 3, LA-UR-96-858, Los Alamos National Laboratory, Los Alamos, New Mexico.

- Document contains waste type summaries, primary chemical compound/analyte and radionuclide estimates for sludge, supernatant, and solids, as well as SMM, TLM, and individual tank inventory estimates.

Agnew, S. F., 1995, Letter Report: Strategy for Analytical Data Comparisons to HDW Model, (external letter \#CST-4:95-sfa272 to S. J. Eberlein, Westinghouse Hanford Company, September 28), Los Alamos National Laboratory, Los Alamos, New Mexico.

- Document contains proposed tank groups based on TLM, and statistical method for comparing analytical information to HDW predictions.

Allen, G. K., 1976, Estimated Inventory of Chemicals Added to Underground Waste Tanks, 1944 - 1975, ARH-CD-601B, Atlantic Richfield Hanford Company Operations, Richland, Washington.

- Document contains major components for waste types, and some assumptions

Allen, G. K., 1975, Hanford Liquid Waste Inventory As Of September 30, 1974, ARH-CD-229, Atlantic Richfield Hanford Company Operations, Richland, Washington.

- Document contains major components for waste types, and some assumptions 
Brevick, C. H., L. A. Gaddis, and W. W. Pickett, 1996, Historical Tank Content Estimate for the Southwest Quadrant of the Hanford 200 Areas, WHC-SD-WM-ER-352, Rev OB, Westinghouse Hanford Company, Richland, Washington.

- Document contains summary information from the supporting document for Tank Farms S, SX, and $U$ as well as in-tank photo collages and the solid (including the interstitial liquid) composite inventory estimates.

Hill, J. G., G. S. Anderson, and B. C. Simpson, 1995, The Sort on Radioactive Waste Type Model: A Method to Sort Single-Shell Tanks into Characteristic Groups, PNL-9814, Rev. 2, Pacific Northwest Laboratory, Richland, Washington.

- Document statistically groups Hanford 200 Area Tanks by waste type and provides nominal compositions for the waste types.

Kupfer, M. J., 1996, Interim Report: Best Basis Total Chemical and Radionuclide Inventories in Hanford Site Tank Waste, WHC-SD-WM-TI-740, Rev. B-Draft, Westinghouse Hanford Company, Richland, Washington.

- Document contains a global component inventory for 200 Area waste tanks, currently inventoried are 14 chemical and 2 radionuclide components.

Schmittroth, F. A., 1995, Inventories for Low-Level Tank Waste, WHC-SD-WM-RPT-164, Rev. 0, Westinghouse Hanford Company, Richland, Washington.

- Document contains a global inventory based on process knowledge and radioactive decay estimations using ORIGEN2. $\mathrm{Pu}$ and $\mathrm{U}$ waste contributions are taken at $1 \%$ of the amount used in processes. Also compares information on Tc-99 from both ORIGEN2 and analytical data.

IIIb. Compendium of data from other sources physical and chemical

Agnew, S.F., John G. Watkin, 1994, Estimation of Limiting Solubilities for Ionic Species in Hanford Waste Tank Supernates, LAUR-94-3590, Los Alamos National Laboratory, Los Alamos, New Mexico.

- Document gives solubility ranges used for key chemical and radionuclide components based on supernatant sample analyses. 
Baldwin, D. L., Stromatt, R. W., and Winters, W. I., 1994, Comparative Study of Total Organic Carbon Methods for High-Level Mixed Waste, PNL-SA-23718, Pacific Northwest National Laboratory, Richland, Washington.

- Document contains comparison of hot persulfate oxidation, furnace combustion, and UV-catalyzed persulfate oxidation TOC method analyses using numerous tank samples.

Bratzel, D. R., 1995, Headspace Gas and Vapor Characterization Summary for the 43 Vapor Program Suspect Tanks, WHC-SD-WM-ER-514, Rev. 1A, Westinghouse Hanford Company, Richland, Washington.

- Document summarizes vapor sampling analytical results for 43 single-shell tanks for safety and worker breathing considerations.

Brevick, C. H., L. A. Gaddis, and E. D. Johnson, 1995, Tank Waste Source Term Inventory Validation, Volumes $I \& I I$, WHC-SD-WM-ER-400, Rev. 0, Westinghouse Hanford Company, Richland, Washington.

- Document contains a quick reference to sampling information in spreadsheet or graphical form for 23 chemicals and 11 radionuclides for all the tanks.

Brevick, C. H., L. A. Gaddis, and E. D. Johnson, 1994, Supporting Document for the Historical Tank Content Estimate for U Tank Farm, WHC-SD-WM-ER-325, Rev. 0, Westinghouse Hanford Company, Richland, Washington.

- Document contains summary tank farm and tank write-ups on historical data and solid inventory estimates as well as appendices for the data. The appendices contain the following information: level history, temperature graphs, surface level graphs, a cascade/dry well chart, riser configuration drawings and tables, in-tank photos, and tank layer model information.

DeLorenzo, D. S., J. H. Rutherford, D. J. Smith, D. B. Hiller, K. W. Johnson, and B. C. Simpson, 1994, Tank Characterization Reference Guide, WHC-SD-WM-TI-648, Rev. 0, Westinghouse Hanford Company, Richland, Washington.

- Document summarizes issues surrounding the characterization of nuclear wastes stored in Hanford Site waste tanks. 
Hanlon, B.M., 1996, Tank Farm Surveillance and Waste Status Summary Report for September 30, 1996, WHC-EP-0182-102, Westinghouse Hanford Company, Richland, Washington.

- Document contains a monthly summary of: fill volumes, Watch List tanks, occurrences, integrity information, equipment readings, equipment status, tank location, and other miscellaneous tank information.

Husa, E. I., D. A. Lauhala, and L. A. Tusler, 1995, Hanford Waste Tank Preliminary Dryness Evaluation, WHC-SD-WM-TI-703, Rev. 0, Westinghouse Hanford Company, Richland, Washington.

- Document gives assessment of relative dryness between tanks.

Husa, E. I., R. E. Raymond, R. K. Welty, S. M. Griffith, B. M. Hanlon, R. R. Rios, and N. J. Vermeulen, 1993, Hanford Site Waste Storage Tank Information Notebook, WHC-EP-0625, Westinghouse Hanford Company, Richland, Washington.

- Document contains in-tank photos as well as summaries on the tank description, leak detection system, and tank status.

Leach, C. E., and S. M. Stahl, 1993, Hanford Site Tank Farm Facilities Interim Safety Basis, WHC-SD-WM-ISB-001, Rev. 0, Westinghouse Hanford Company, Richland, Washington.

- Document provides ready reference to the tank farms safety envelope.

Shelton, L. W., 1996, Chemical and Radionuclide Inventory for Single and Double Shell Tanks, (internal memorandum 74A20-96-30 to

D. J. Washenfelder, February 28), Westinghouse Hanford Company, Richland, Washington.

- Memorandum contains a tank inventory estimate based on analytical information.

Shelton, L. W., 1995, Chemical and Radionuclide Inventory for Single and Double Shell tanks, (internal memorandum \#75520-95-007 to R. M. Orme, August 8), Westinghouse Hanford Company, Richland, Washington.

- Memorandum contains a tank inventory estimate based on analytical information. 
Shelton, L. W., 1995, Radionuclide Inventories for Single and Double Shell Tanks, (internal memorandum \#71320-95-002 to F. M. Cooney, February 14), Westinghouse Hanford Company, Richland, Washington.

- Memorandum contains a tank inventory estimate based on analytical information.

Van Vleet, R. J., 1993, Radionuclide and Chemical Inventories for the Single Shell Tanks, WHC-SD-WM-TI-565, Rev. 1, Westinghouse Hanford Company, Richland, Washington.

- Document contains selected sample analysis tables prior to 1993 for singleshell tanks. 


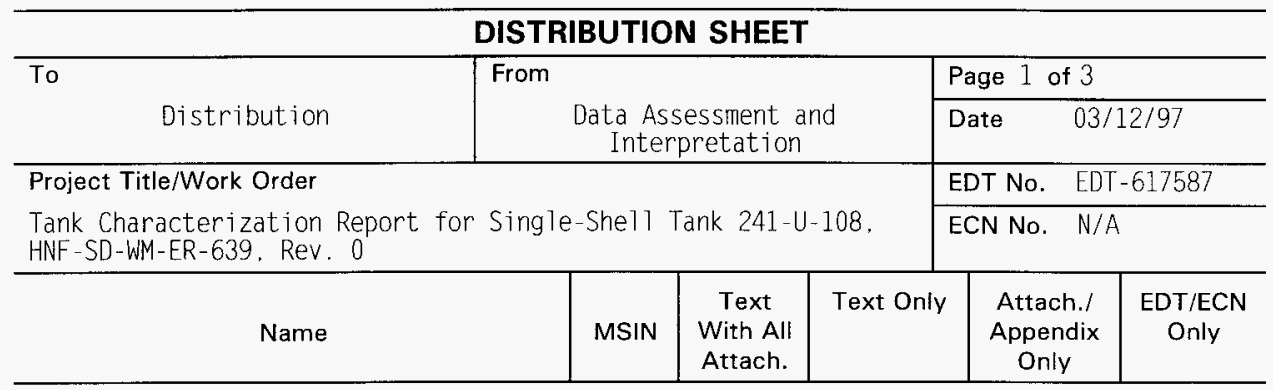

OFFSITE

Sandia National Laboratory

P. 0. Box 5800

MS-0744. Dept. 6404

Albuquerque, NM 87815

D. Powers

$x$

Nuclear Consulting Services Inc.

P. 0. Box 29151

Columbus, $\mathrm{OH}$ 43229-01051

J. L. Kovach

Chemical Reaction Sub-TAP

P. 0. Box 271

Lindsborg, KS 67456

B. C. Hudson

$X$

Tank Characterization Panel

Senior Technical Consultant

Contech

7309 Indian School Road

Albuquerque, NM 87110

J. Arvisu

$x$

SAIC

$\frac{\mathrm{A}}{20300}$ Century Boulevard, Suite 200-B

Germantown. MD 20874

H. Sutter

$x$

Los Al amos Laboratory

CST-14 MS-J586

p. 0. Box 1663

Los Alamos. NM 87545

S. F. Agnew

$x$ 


\section{DISTRIBUTION SHEET}

\begin{tabular}{|c|c|c|c|c|c|}
\hline \multirow{2}{*}{$\begin{array}{l}\text { To } \\
\text { Distribution }\end{array}$} & \multirow{2}{*}{\multicolumn{3}{|c|}{$\begin{array}{r}\text { Data As } \\
\text { Inter }\end{array}$}} & \multicolumn{2}{|l|}{ Page 2 of 3} \\
\hline & & & & \multicolumn{2}{|c|}{$03 / 12 / 97$} \\
\hline \multicolumn{4}{|l|}{ Project Title/Work Order } & \multicolumn{2}{|c|}{ EDT No. EDT-617587 } \\
\hline \multicolumn{4}{|c|}{$\begin{array}{l}\text { Tank Characterization Report for Single-Shell Tank 241-U-108, } \\
\text { HNF-SD-WM-ER-639. Rev. 0 }\end{array}$} & \multicolumn{2}{|l|}{ ECN No. $\quad N / A$} \\
\hline Name & MSIN & $\begin{array}{l}\text { Text } \\
\text { With All } \\
\text { Attach. }\end{array}$ & Text Only & $\begin{array}{l}\text { Attach./ } \\
\text { Appendix } \\
\text { Only }\end{array}$ & $\begin{array}{l}\text { EDT/ECN } \\
\text { Only }\end{array}$ \\
\hline
\end{tabular}

Los Alamos Technical Associates

T. T. Tran

B1-44 $\quad X$

Tank Advisory Panel

102 Windham Road

Oak Ridge. TN 37830

D. 0. Campbel1

\section{ONSITE}

Department of Enerqy - Richland Operations

$\begin{array}{lll}\text { J. F. Thompson } & \text { S7 }-54 & X \\ \text { W. S. Liou } & 57-54 & X \\ \text { J. A. Poppiti } & 57-54 & X \\ \text { N. W. WiTlis } & \text { S7-54 } & X\end{array}$

DE\&S Hanford, Inc.

R. J. Cash

S7 -14

W. L. Cowley

$\mathrm{R} 2-54$

G. L. Dunford

A2 -34

G. D. Johnson

S7 -14

J. E. Meacham

$57-14 \quad \hat{x}$

Fluor Daniel Northwest

J. L. Stroup

S3-09 $\quad x$

Lockheed Martin Hanford. Corp.

K. E. Bell

K. M. Hodgson

T. J. Kelley

L. M. Sasaki

B. C. Simpson

L. R. Webb

ERC (Environmental Resource Center)

Tank Characterization Resource Center

R2-12

$\mathrm{HO}-34$

S7 - 21

R2 -12

R2 - 12

R2 -12

R1-51

R2-12

$x$
$x$
$x$
$x$

Lockheed Martin Services. Inc.

B. G. Lauzon

R1 -08

Central Files

A3-88

EDMC

H6-08

$x$
$X$
$x$
$x$
$x$


DISTRIBUTION SHEET

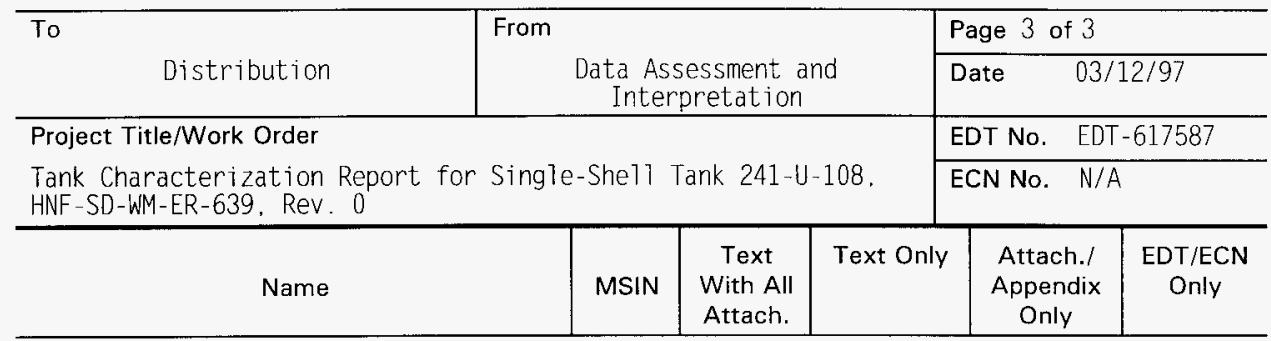

Numatec Hanford Corporation

J. S. Garfield

J. S. Hertzel

D. L. Lamberd

H5-49

H5-61

H5-61

$x$
$x$
$x$

Pacific Northwest Nationa 7 Laboratory

A. F. Noonan

K9-91 X

Rust Federal Services of Hanford, Inc. C. T. Narquis

T6-16 $\quad x$

SGN Eurisys Services Corp.

D. B. Engelman

L6-37 X

